Development and evolution of the skull-neck boundary: Insights from amphibian model species

$$
\text { by }
$$

Jade Atkins

A thesis submitted to the Faculty of Graduate and Postdoctoral Affairs in partial fulfillment of the requirements for the degree of

Doctor of Philosophy

in

Earth Sciences

Carleton University

Ottawa, Ontario

(C)2021, Jade Atkins 


\section{Abstract}

The history of life is marked by near constant morphological transformations, preserved in part by the fossil record. Unfortunately, the developmental mechanisms that drove many key morphological transformations are not well understood. In order to study these morphological transformations and their underlying developmental mechanisms, an integrative approach synthesizing data from palaeontological, phylogenetic, and developmental sources is required. Here, I have focused on improving our understanding of one such transformation, the morphology and development of the skull-neck boundary in lissamphibians. To complete this goal, I tested two hypotheses. First, that the skull-neck boundary and composition of the occiput observed in extant lissamphibians is the result of a secondary reduction compared to their fossil relatives. Second, that changes in Hox gene expression domains can cause homeotic transformations of skull-neck boundary structures in two lissamphibian model organisms, Ambystoma mexicanum (salamanders) and Xenopus laevis (frogs). I found using phylogenetic analyses and ancestral state reconstructions that the lissamphibian occipital morphology is secondarily derived when compared to their fossil relatives. Then, I described the development of the skull, focusing on the occiput, in A. mexicanum and X. laevis using cell-lineage tracing techniques to track somitic contributions to the skull, hypoglossal nerve complex morphological data, and traditional whole-mount cartilage and bone staining techniques. Finally, I perturbed Hox gene expression patterns to produce homeotic transformations via exogenous retinoic acid and a retinoic acid inhibitor (citral) and described the resulting skull morphology. I provided evidence that homeotic transformations of skull-neck boundary structures could be induced via changing retinoic acid concentration in the developing embryo: first with 
the position of the hypoglossal nerve complex relative to skeletal structures along the anterior-posterior axis and second with GFP-labelled somite cell-lineage tracing. The resulting morphologies indicate that it is likely that changing Hox gene expression domains resulted in the unique lissamphibian morphology at the occiput and the derived position of their skull-neck boundary. This research contributes to our understanding of the evolution of the unique lissamphibian skull-neck morphology. My integrative approach has helped to elucidate the relationship between morphological transformations and the developmental processes underlying them. 


\section{Statement of Contributions}

I published Chapter 2 in PLoS One in 2019 with co-authors Hillary Maddin and Robert Reisz. I conceived and designed the study with Hillary Maddin. I received help learning phylogenetic analyses programs from Ryan Paterson and writing my R code for my ancestral state reconstructions from Danielle Fraser. I then conducted all the analyses and wrote the manuscript. Hillary Maddin and Robert Reisz provided helpful comments to various versions of the manuscript.

Chapter 3 contains two skull growth series, one for Ambystoma mexicanum and one for Xenopus laevis. These growth series were developed after multiple discussions with Hillary Maddin about the lack of normal skull development literature that suited the needs of this thesis. I published the growth series for A. mexicanum in Developmental Dynamics in 2020 with my colleagues Alanna Cantelon, Laurent Houle, and Hillary Maddin. Alanna took care of all of our animals, bone and cartilage stained them, and provided helpful comments on the figures. Laurent provided helpful comments to the discussion. Hillary Maddin helped with the stage descriptions, the figures, and provided comments during all stages of writing. The growth series for $X$. laevis is not currently submitted anywhere nor is it published. However, it is in the stages of final edits with colleagues Alanna Cantelon, Dana Korneisel, Erin MacKenzie, and Hillary Maddin. Alanna took care of our animals, completed the bone and cartilage staining protocols and took photos of stages for us. Dana and Erin helped with the final figures, stage descriptions, and discussion. Hillary Maddin provided comments to several versions of this manuscript. The hypoglossal nerve complex data and the cell-lineage tracing data has not been published nor is it currently submitted anywhere. 
Chapter 4 is not currently published nor is it submitted. This chapter was conceived with Hillary Maddin. I collected all the data and performed all the analyses with the exception of two donated datasets containing GFP-position somite transplant data for $A$. mexicanum from Hillary and Nadine Piekarski. Alanna Cantelon took care of the animals and conducted the bone, nerve, and cartilage whole-mount staining protocols.

Publications related to this thesis:

Atkins, J. B., Reisz, R. R. and Maddin, H. C. (2019). Braincase simplification and the origin of lissamphibians. PLoS One, 14, e0213694.

Atkins, J. B., Houle, L., Cantelon, A. S., and Maddin, H. C. (2020). Normal development in Ambystoma mexicanum: A complementary staging table for the skull based on Alizarin red S staining. Developmental Dynamics, 249(5), 656-665 


\section{Acknowledgements}

First and foremost, it is important that I acknowledge that for six years I was a guest on unceded Algonquin territory as it is the land of the Algonquin nation that Ottawa, and Carleton University, resides on.

For my second chapter, I wish to thank and acknowledge Danielle Fraser for her immense patience in teaching me how to use R for my ancestral state reconstructions. I also want to thank Ryan Paterson for his patience in teaching me how to use PAUP and MrBayes. Thank you to Rainer Schoch for sending me an advance copy of his manuscript and matrix, as it was the basis for my own phylogenetic analyses.

For my third chapter, I owe an immense debt of gratitude to Alanna Cantelon for her dedication and care to the Maddin Lab animals. Alanna also tirelessly worked our bone, cartilage, and nerve stain protocols and her efforts allowed me to include such amazing figures for this chapter and my fourth chapter. Thank you to Laurent Houle for helping out with the Ambystoma mexicanum staging table during his summer in our lab. For the Xenopus laevis staging table, I wish to thank Dana Korneisel and Erin MacKenzie for their help with the results and discussion sections and by saving the day with their amazing ability to make gorgeous figure panels from virtually nothing but a good photo. Additional thanks to Alanna Cantelon and Sara Hassan for taking some of those good photos.

For my fourth chapter, I again want to thank Alanna Cantelon for working so hard to keep my experimental animals alive. I want to thank all the members of the Ekker Lab at the University of Ottawa for being so welcoming and helpful during my time there trying to make in situ hybridization probes. I am especially grateful to Gary Hatch, Khang Hua, and Hellen Weinshutz Mendes for their help in teaching me protocols and troubleshooting 
with me. I also want to thank Nadia Fröbisch and Christian Mitgutsch for all their help troubleshooting an in situ hybridization protocol for axolotl from all the way in Germany! Additional thanks to Tetsuto Miyashita for all his help virtually troubleshooting these difficult protocols during a pandemic! I owe Erin MacKenzie and Dana Korneisel so much, Erin for tirelessly sectioning while I worked on troubleshooting our in situ hybridization probes for our difficult genes, and Dana for constantly running in situ hybridization protocol after protocol until we found something that worked for our lab. While these datasets ultimately were not ready in time to be included in this thesis, their hard work still needs to be recognized.

In general, I have to thank past and present members of the Maddin Lab for their friendship and support during time at Carleton. Alanna Cantelon for virtually everything in the early days of animal care and during the worst of the pandemic. You were often my rock during difficult times in the lab. Fadi Naddeh and Mat Roloson for all their moral support and help with taking care of our animals. Erin MacKenzie and Dana Korneisel for all their help, especially during the pandemic. Gabby Adams for being a great colleague, Tom Dudgeon for being an excellent sounding board, and Brad McFeeters for all the Scrabble games and long chats about methodologies. Misha Whittingham, you have been a great roommate and probably the best person to weather this pandemic with!

I have many, many people to thank in Carleton Earth Sciences, but thank you especially to Geoff Pignotta for teaching me absolutely everything I know about first year geology and to Beth McLarty-Halfkenny for being an excellent mentor, providing me with so many opportunities for outreach, and for always giving me perspective. 
Additionally, thank you to my many friends outside of the lab who helped to keep me sane during this degree. Especially to Erin Bethell, Kaeli Cecchetto, Brigid Christison, Trinda Dunlop, Sabastien Dyer, Yara Haridy, Stacey Henderson, Zoe Landry, Cal Langley, Nico Kastek, Ronnie Mazzella, Rachel Nottrodt, Nkechi Oruche, Sam Pokrywa, Nina Shu, Tanya Snow, Mike Thompson, Balie Walker, and Lauren Wu. You have all been amazing and I'm sorry for how frazzled and stressed I've been since approximately 2015 !

To my family, both here in Ottawa and afar, especially my parents, Wanita and Martin, and my brothers, thank you for being so supportive as I cried about the delays and celebrating even the smallest victories with me. I am so incredibly lucky to have such great people in my life. I am so excited to share this final victory with you, and I thank you for encouraging me to always follow my dreams, even if that has taken me down a strange and winding path.

To Robert Reisz, thank you for accepting to be my co-supervisor, even though I have veered heavily away from the fossil side of things.

Finally, to my primary supervisor, Hillary Maddin, I am so incredibly grateful for this opportunity and so incredibly lucky that you have been my supervisor these past six years. We started this journey together six years ago, and we had no idea the many ups and downs that would occur, from a delayed lab to a global pandemic. Thank you for all your moral support, the craft nights, the quick chats when I was stressed and sobbing about all my lab failures. Thank you for being patient with my writing, and with how stressed I was leading up to finishing this beastly document. It has not been an easy road, and I am so happy that it is finally over, but also excited for opportunities to collaborate in the future. 


\section{Dedication}

To my brother, Zachary.

From reluctantly collecting newts with you as children to devoting six years of my life to studying amphibians. I wish you could see me now. 


\section{Table of Contents}

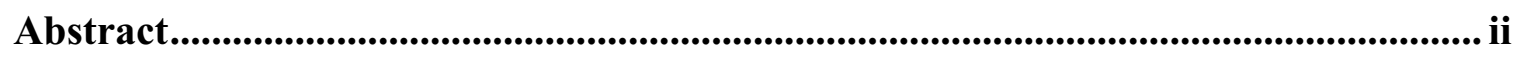

Statement of Contributions ........................................................................................... iv

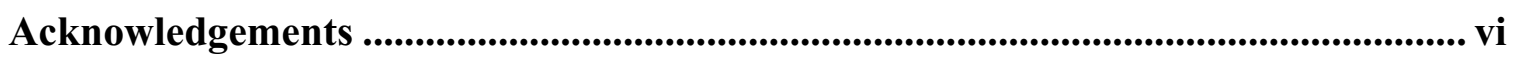

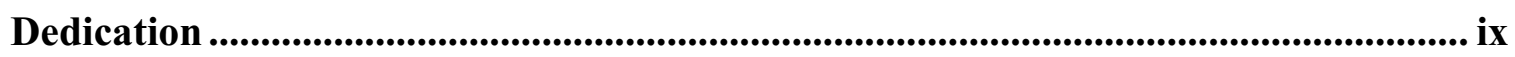

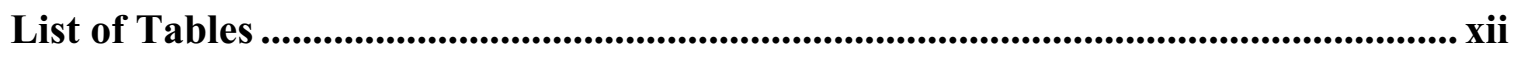

List of Figures.................................................................................................................... xiv

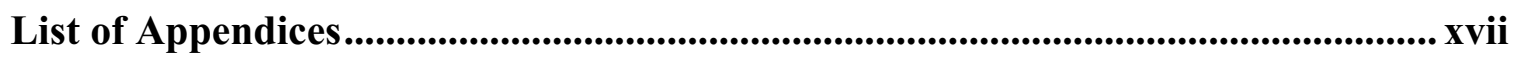

Chapter 1: Introduction ......................................................................................................... 1

1.1: Developmental origins of the vertebrate skull .................................................. 1

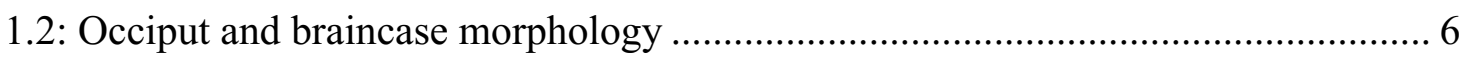

1.3: The developmental mechanisms underlying axial patterning ............................. 12

1.4: Project goals and thesis outline....................................................................... 16

Chapter 2: Braincase and occipital simplification and the origin of lissamphibians 21

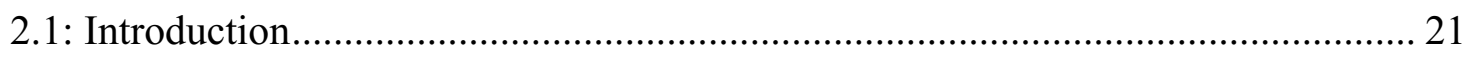

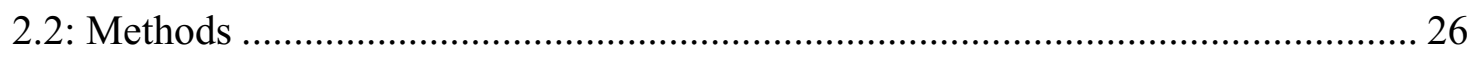

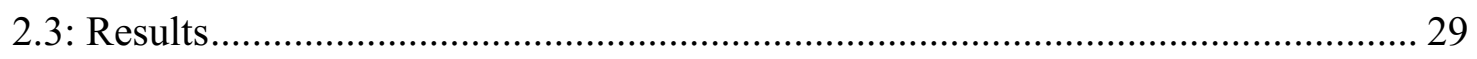

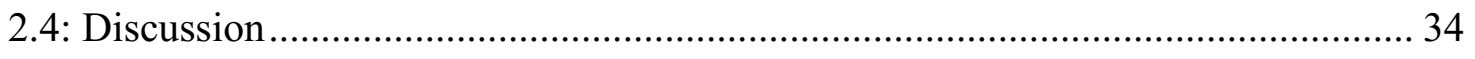

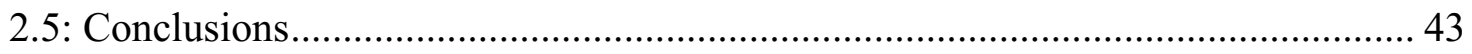

Chapter 3: Normal skull development in two amphibian model organisms, Ambystoma mexicanum (salamander) and Xenopus laevis (frog) ............................... 45

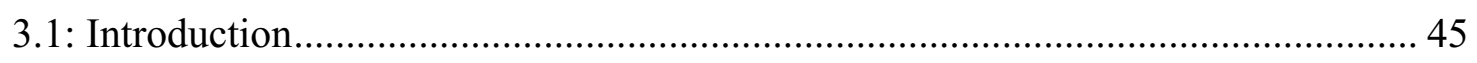

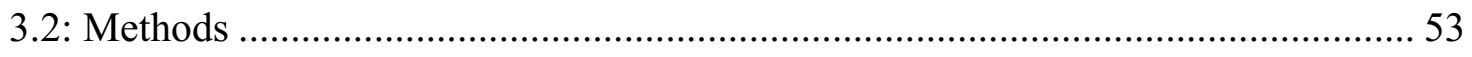

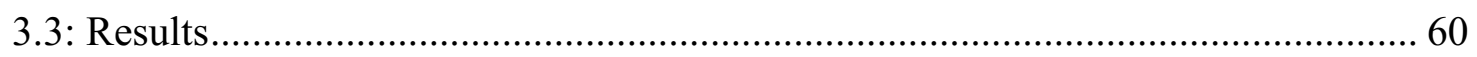

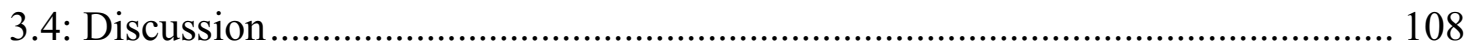

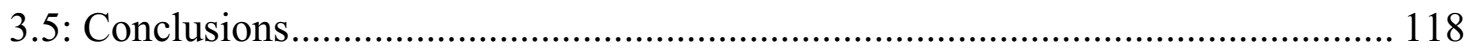

Chapter 4: Weird bois, the experimental effects of perturbed Hox genes on the development of the skull-neck boundary ........................................................................ 120

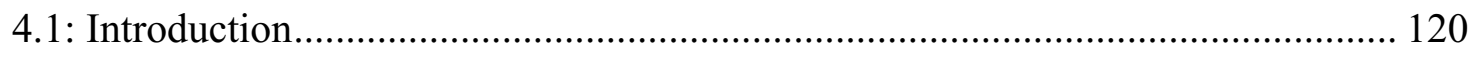


4.2: Methods

4.3: Results.

4.4: Discussion.

4.5: Conclusions

Chapter 5: Discussion ....................................................................................... 171

5.1: The lissamphibian occipital morphology is secondarily derived....................... 172

5.2: The reduced lissamphibian occipital morphology likely arose as the result of shifting Hox gene expression domains

5.3: Heterochrony versus heterotopy

5.4: What drove the anterior Hox gene shift? ................................................... 178

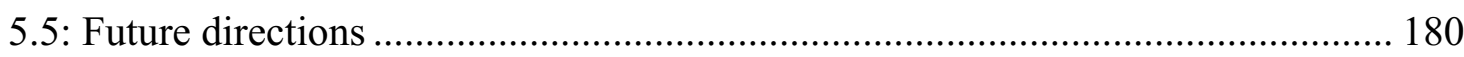

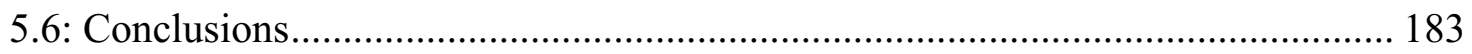

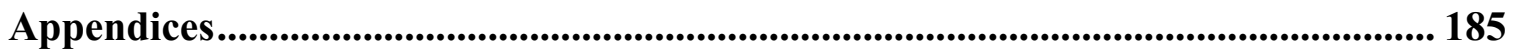

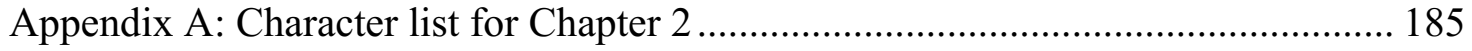

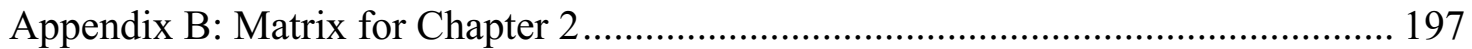

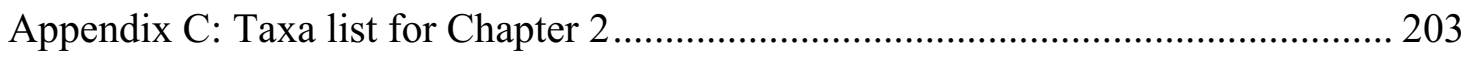

Appendix D: Somite injection specimen numbers for Chapter 3 ........................... 208

Appendix E: Ambystoma mexicanum staging table specimen numbers .................... 209

Appendix F: Xenopus laevis staging table specimen numbers .............................. 210

Appendix G: Ambystoma mexicanum exogenous and inhibited retinoic acid treatment

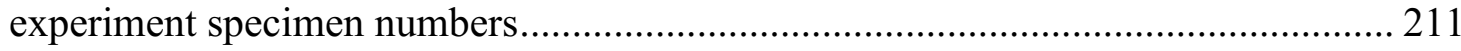

Appendix H: Xenopus laevis exogenous and inhibited retinoic acid treatment experiment specimen numbers.................................................................... 213

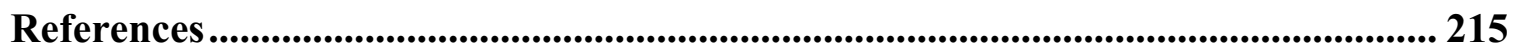




\section{List of Tables}

Table C1. A list of taxa coded for the phylogenetic analyses in Chapter 2..................203

Table D1. Ambystoma mexicanum specimens injected with dextran-fluorescein to track

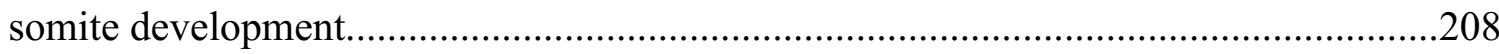

Table D2. Ambystoma mexicanum specimens that underwent GFP-positive somite

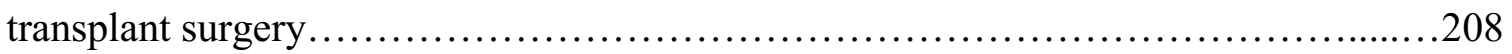

Table D3. Xenopus laevis specimens injected with dextran-fluorescein to track somite

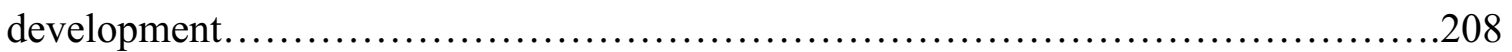

Table E1. Ambystoma mexicanum specimens whole-mount cartilage and bone stained for the staging series.

.209

Table E2. Ambystoma mexicanum specimens whole-mount nerve stained. .209

Table F1. Xenopus laevis specimens whole-mount cartilage and bone stained for the staging series

Table F2. Xenopus laevis specimens whole-mount nerve stained 210

Table G1. Total number of Ambystoma mexicanum experimental animals exposed to exogenous retinoic acid.

Table G2. Total number of Ambystoma mexicanum experimental animals where retinoic acid was inhibited via citral.

Table G3. Total number of Ambystoma mexicanum with GFP-positive somite transplants experimental animals exposed to exogenous retinoic acid or citral

Table H1. Total number of Xenopus laevis experimental animals exposed to exogenous retinoic acid .213 
Table H2. Total number of Xenopus laevis experimental animals where retinoic acid was

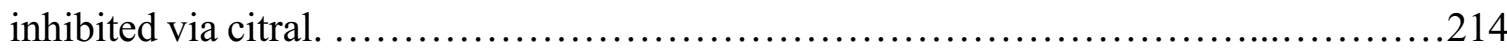




\section{List of Figures}

Figure 1.1. Schematic of a chick embryo in dorsal view during somitogenesis..................3

Figure 1.2. Summary of alternate hypotheses on the evolution of occipital somite

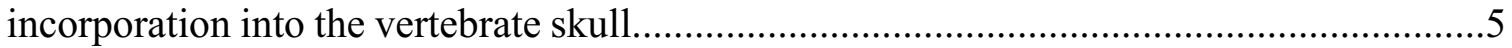

Figure 1.3. A comparison of the braincase and occiput of extinct and extant

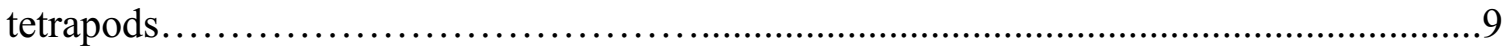

Figure 1.4. Schematic synthesized from the literature showing where the hypoglossal (XII) exits with respect to the brain or spinal column................................. 10

Figure 1.5. Schematic of axial somites, their fate and associated Hox gene expression

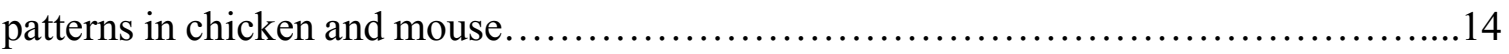

Figure 1.6. Retinoic acid causes homeotic transformations along the anteriorposterior axis .15

Figure 2.1. The three hypotheses for extant lissamphibians and their closest relatives....22

Figure 2.2. A simplified phylogenetic tree for Temnospondyli......................24

Figure 2.3. The results of the phylogenetic analyses of Dissorophoidea.................30

Figure 2.4. Summary of the ancestral character state reconstructions of the braincase characters.

Figure 3.1. Examples of embryos used for somite lineage tracing experiments. .56

Figure 3.2. A summary of somites that contribute to the muscles, chondrocranium and anteriormost vertebral structures in $A$. mexicanum specimens.

Figure 3.3. A summary of somites hypothesized to contribute to the muscles and occiput in Xenopus laevis. 
Figure 3.4. Stages 45, 47, 49, and 56, Ambystoma mexicanum cartilaginous skull. .78

Figure 3.5. Stages 45 to 49, Ambystoma mexicanum ossified skull....................79

Figure 3.6. Stages 50 to 53, Ambystoma mexicanum ossified skull....................80

Figure 3.7. Stages 54 to 57 , Ambystoma mexicanum ossified skull...................81

Figure 3.8. Development of the chondrocranium, stages 41 to 48, in Xenopus laevis.....101

Figure 3.9. Development of the chondrocranium, stages 49 to 53, in Xenopus laevis....102

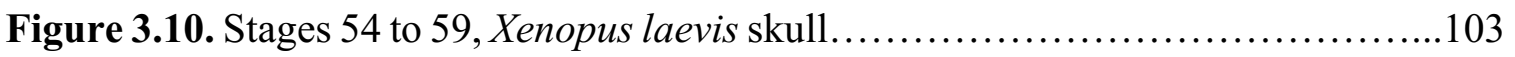

Figure 3.11. Stages 60 to 63, Xenopus laevis skull............................... 104

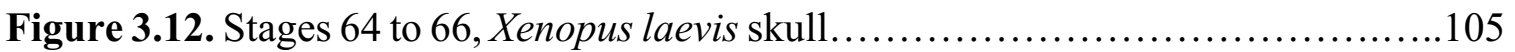

Figure 3.13. A stage 66 Xenopus laevis specimen showing the two rows of teeth present in the maxilla and premaxilla................................................. 106

Figure 3.14. Ambystoma mexicanum and Xenopus laevis specimens stained with Sudan Black to visualize nerves. .107

Figure 4.1. The Clock and Wavefront model showing the temporal sequence of somite

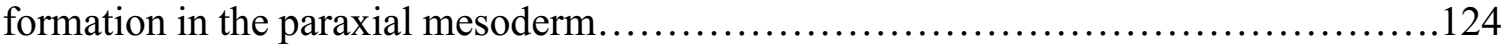

Figure 4.2. How retinoic acid interacts with Hox genes............................ 125

Figure 4.3. Ambystoma mexicanum specimens exposed to exogenous retinoic acid.....139

Figure 4.4. Older Ambystoma mexicanum specimens exposed to exogenous retinoic acid.

Figure 4.5. Ambystoma mexicanum specimens where normal retinoic acid expression within the embryo was inhibited with citral.

Figure 4.6. Older Ambystoma mexicanum specimens where normal retinoic acid expression within the embryo was inhibited with citral. 
Figure 4.7. Ambystoma mexicanum specimens, nerve stained .144

Figure 4.8. Unpublished dataset of whole-mount Ambystoma mexicanum specimens with GFP-transplanted somites. 146

Figure 4.9. Unpublished dataset of sectioned Ambystoma mexicanum specimens with GFP-transplanted somites 148

Figure 4.10. Xenopus laevis experimental specimens external morphology compared to the morphology of the control specimens................................... 150

Figure 4.11. Xenopus laevis specimens exposed to exogenous retinoic acid. .152 Figure 4.12. Xenopus laevis where normal retinoic acid expression within the embryo was inhibited with citral. 154

Figure 4.13. Xenopus laevis specimens, nerve stained. .155

Figure 4.14. Schematic showing normal contributions to the occipital arch and the homeotic transformations caused in the present study .158 


\section{List of Appendices}

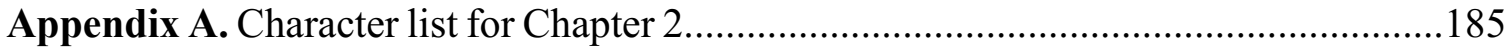

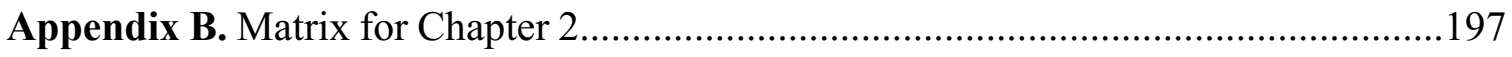

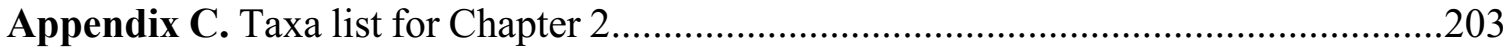

Appendix D. Somite injection numbers for Chapter 3 ….........................................20

Appendix E. Ambystoma mexicanum staging table specimen numbers............................209

Appendix F. Xenopus laevis staging table specimen numbers.....................................210

Appendix G. Ambystoma mexicanum exogenous and inhibited retinoic acid experiments

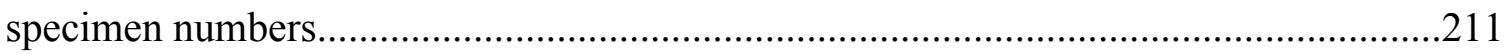

Appendix H. Xenopus laevis exogenous and inhibited retinoic acid experiments specimen

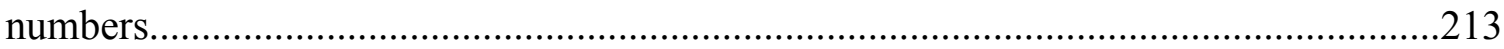




\section{Chapter 1: Introduction}

The fossil record reveals a long history of morphological transformations throughout evolutionary time. While these morphological transformations are captured in the fossil record, less well understood are the mechanisms underlying such transformations. To study the underlying mechanism of a transformation an integrative approach is required combining developmental and paleontological data. Such integrative approaches have improved our understanding of many pivotal transformations (e.g., fin-to-limb) and their underlying mechanisms, whereas others, such as the evolution of the skull-neck boundary in tetrapods, remain poorly understood.

\section{1: Developmental origins of the vertebrate skull}

In vertebrates, the skull is composed of two or three units, the neurocranium (or chondrocranium), the viscerocranium, and the dermatocranium, the latter of which may or may not be present (Hanken and Thorogood, 1993; Hall 2005; Kardong, 2015). The neurocranium may be composed of cartilage only (e.g., in sharks) or variably ossified as endochondral bone - bone that forms by replacing cartilage during development (Hall, 2005). The neurocranium primarily protects the brain and sense organs (Hanken and Thorogood, 1993; Kuratani, 2005). The viscerocranium forms the branchial arches, the most anterior of which was modified during vertebrate evolution to form the jaws of gnathostomes (Hanken and Thorogood, 1993; Hall, 2005; Kuratani, 2005). The dermatocranium is composed exclusively of dermal bone - bone that forms without a cartilaginous precursor (Hanken and Thorogood, 1993; Hall, 2005). Several vertebrates 
(e.g., agnathans, sharks, etc.) do not have a dermatocranium (Hanken and Thorogood, 1993); however, the vast majority do.

In vertebrates, the developing skull receives contributions from the neural crest and mesoderm (e.g., Couly et al., 1993; Hall, 2005; Gross and Hanken, 2008; Gilbert, 2010; Piekarski et al., 2014; Maddin et al., 2016). While there have been numerous important studies that investigate the contributions of the neural crest to the skull (e.g., numerous studies outlined in Hall, 2005 as well as Kuratani, 2005; Matsuoka et al., 2005; Gross and Hanken, 2008; Mitgutsch et al., 2008; Hanken and Piekarski, 2013), there have been far fewer studies investigating the contribution of the mesoderm to the skull. The mesoderm contributes to the posterior, post-otic portion of the skull, forming the posteriormost bone of the cranial vault (i.e., the parietal) and the bones of the occiput (Couly et al., 1993; Gross and Hanken, 2008; Piekarski et al., 2014; Maddin et al., 2016). The occiput defines the posterior limit of the skull at the point of separation between the occipital condyles and the first vertebra of the vertebral column (Ferguson and Graham, 2004; Kardong, 2015). This is the skull-neck boundary. Of particular interest to the story of skull-neck boundary evolution are thus the bones of the occiput, which are derived specially from the paraxial mesoderm.

The bones of the occiput, along with those of the vertebral column, are derived from transient paraxial mesodermal structures called somites (Hall, 2005; Olsson et al., 2005; Gomez and Pourquié, 2009; Gilbert, 2010). Somites organize the segmental patterning of the vertebrate embryo along the anterior-posterior axis and determine the migration pathways of the neural crest and spinal nerves, making them important developmental structures to a variety of anatomical systems (Keynes and Stern, 1984; Kalcheim, 2011; 
Kuratani et al., 2018). During somitogenesis, somites begin developing in the anteriorposterior direction in a rhythmic fashion, where somites in the anterior region of the embryo form earlier than somites in the posterior region (Gomez and Pourquié, 2009). Somites start out as loose clusters of mesodermal cells within the bands of paraxial mesoderm on either side of the neural tube (Duband et al., 1987). The mesodermal cells that will become somites first organize into whorls, then they aggregate, and undergo epithelization (Duband et al., 1987). The end product of somitogenesis is the formation of serially-arranged blocks of condensed, paraxial mesoderm along the anterior-posterior axis on either side of the neural tube - the somites (Figure 1.1). Following somitogenesis, the somites differentiate into the dermatome, myotome, and sclerotome (Fleming et al., 2015). The dermatome and myotome go on to differentiate into dermis and skeletal muscles, respectively, whereas the sclerotome differentiates into cartilage and then bone (Fleming et al., 2015).

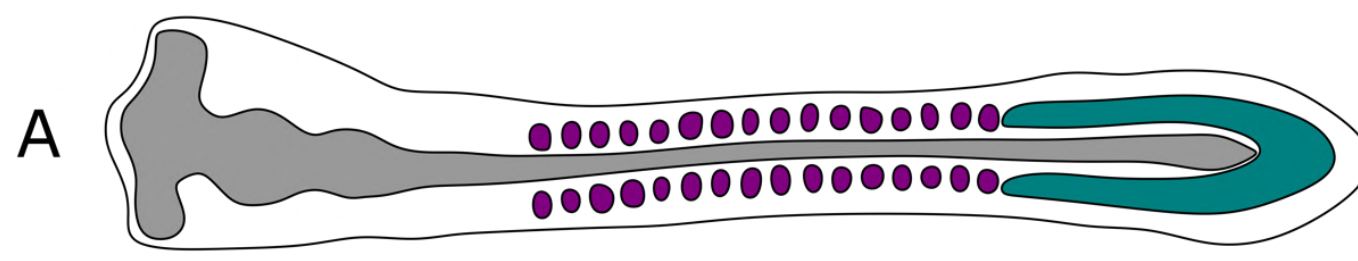

Figure 1.1. Schematic of a chick embryo in dorsal view during somitogenesis. Somites (purple) form sequentially on either side of the neural tube (grey) from the anterior (A) to posterior $(\mathrm{P})$ direction. In this chick specimen, the posteriormost somites have not yet formed and presomitic paraxial mesoderm (green) is present in the posterior, tail region of the embryo. Modified from Gomez and Pourquié (2009).

The number of somites that are incorporated into the occiput of the skull has been the subject of study for a long time up until today (e.g., Platt, 1897; Hunter, 1935; de Beer, 1937; Couly et al., 1993; Burke et al., 1995; Kuratani et al., 1999; Handrigan and 
Wassersug, 2007; Kuratani and Ota, 2008; Maddin et al., 2020). Early research using traditional techniques of histology and whole-mount embryology showed that agnathans (the jawless fishes) do not incorporate any somites into the head (Kuratani et al., 1999), whereas gnathostomes (jawed fishes plus tetrapods) do, and recruit a variable number of somites to form the bones of the occiput (de Beer, 1937). Attempts to understand evolutionary patterns of somite contributions to the skull, such as the study by Augier (1931), suggested that early-diverging vertebrates (e.g., fish and amphibians) incorporate fewer somites, three, into the occiput than later-diverging vertebrates (e.g., amniotes), which he described as incorporating five somites (summarized in Figure 1.2). However, de Beer (1937) later noted that the number of somites that contributed to the occiput is more variable than Augier (1931) suggested, and reported four to eight occipital somites in gnathostome fish, anywhere from one to four occipital somites in amphibians, and generally five or more occipital somites in amniotes, indicating that the stepwise incorporation of somites into the skull between agnathan, lissamphibian (frog, salamanders, and caecilians), and amniote (reptiles and mammals) lineages may not be accurate (Figure 1.2). de Beer (1937) then went on to generate an alternative hypothesis, that the ancestral state for Tetrapoda was to incorporate more somites into the occiput than lissamphibians and that lissamphibians underwent a secondary reduction in their number of occipital somites (Figure 1.2). 

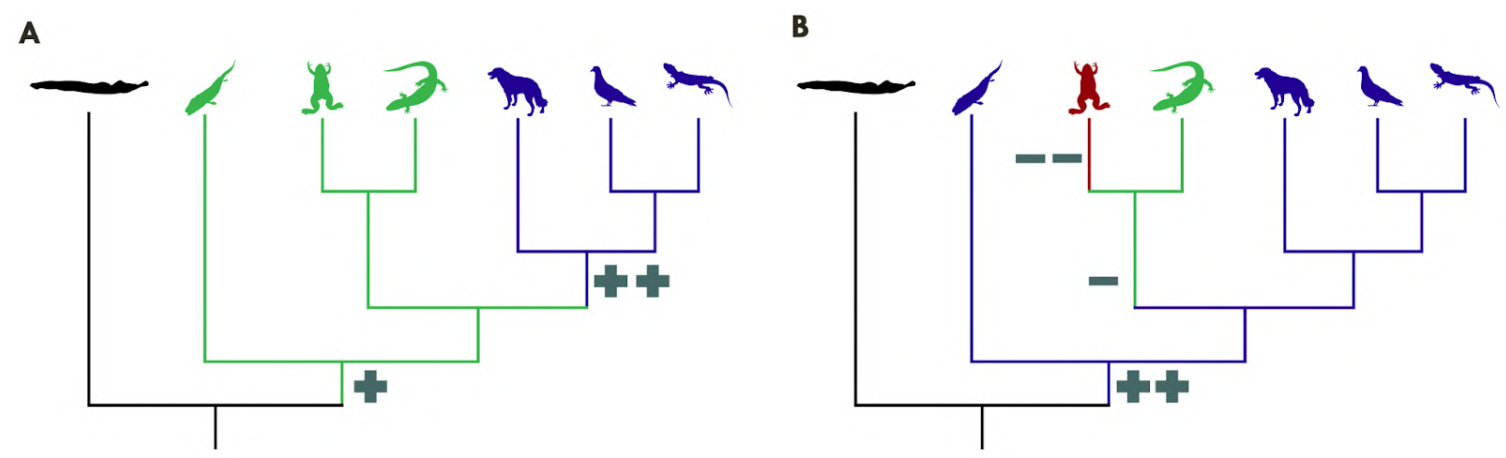

Figure 1.2. Summary of alternate hypotheses on the evolution of occipital somite incorporation into the vertebrate skull. A) Augier's (1931) hypothesis of increased occipitalization, where at the base of Tetrapoda a few somites were recruited into the head $(+)$ and then at the base of Amniota additional somites $(++)$ were further recruited into the head, thus the amphibian condition is the ancestral condition for Tetrapoda. B) de Beer (1937) hypothesized that an amniote-like number of somites were incorporated into the head at the base of Tetrapoda $(++)$, and that amphibians underwent a secondary reduction $(-)$ with frogs even more reduced (- -); thus, the amniote condition is the ancestral condition for Tetrapoda. Silhouettes are from open source PhyloPic.org.

Other studies found variation in occipital somite number amongst amniotes, and even sometimes within the same species: only four in rats (Gasser, 1979); three (Hunter, 1935) or four (Chiarugi, 1889) in rabbits, and anywhere from three to more than four in humans (summarized in O'Rahilly and Meyer, 1979). The discrepancy among previous reports of the number of somites contributing to the skull may be the result of differing definitions of what a somite is and consists of (e.g., Butcher, 1929; Kuratani et al., 1999), the result of short developmental time spans analyzed that may have missed transient anterior somites or somites that become covered by migrating neural crest at various stages of development (e.g., Elliot, 1907; Dawes, 1930; Piekarski, 2009), and the lack of knowledge of somite resegmentation by early researchers (e.g., de Beer, 1937). More recently, modern fate mapping techniques have been applied to certain model species in order to confirm the somitic contribution to the skull in representative taxa (e.g., Couly et al., 1993; Huang et al., 2000; Piekarski and Olsson, 2007; 2014). Five occipital somites 
were confirmed to contribute to the occiput in chicken (Huang et al., 2000) and three occipital somites were counted in Ambystoma mexicanum (Piekarski and Olsson, 2007; 2014). When considering resegmentation, which occurs when somites divide into anterior and posterior halves that then fuse during development to form vertebral elements (e.g., the posterior portion of somite five and the anterior portion of somite six form one vertebral element) the skull-neck boundary then resides within somite five for chickens and within somite three for A. mexicanum. Many taxa remain unstudied in this way, such as frogs, and as single representatives of larger clades, the question of variation in number of occipital somites and thus the location of the skull-neck boundary and its potential evolutionary change among vertebrates remains.

\section{2: Occiput and braincase morphology}

The sclerotomal portions of the occipital somites go on to form the bones of the posterior braincase, specifically the occiput of the skull (de Beer, 1937; Kardong, 2015). Although the identities of the occipital bones that are derived from somites are known in chicken (e.g., Couly et al., 1993; Huang et al., 2000), and the muscles that are derived from somites have been studied and the number of somites contributing to the occiput have been determined in axolotl (e.g., Piekarski and Olsson, 2007; 2014), the specific somitic contribution to the chondrocranium and the bones of the occiput have not been well studied in amphibians.

As with the number of somites that differentiate into occipital somites, the bones that comprise the occiput differ between vertebrate lineages (e.g., de Beer, 1937; Romer, 1946; Romer and Parsons, 1977). In tetrapods, the occiput may consist of up to four bones, including the median supraoccipital, the paired exoccipitals, and the median basioccipital 
(Figure 1.3; Romer, 1946; Romer and Parsons, 1977; Kardong, 2015). The supraoccipital is present in amniotes such as reptiles and mammals, in lepospondyls, which are extinct, Early Carboniferous to Early Permian tetrapods, as well as in some fossil temnospondyls, the lineage that likely contains extant lissamphibians (e.g., Romer, 1946; Berman, 2000; Brusatte et al., 2015; Schoch, 2018), but is absent in fossil taxa closely related to lissamphibians (Schoch, 2018). In extant amphibians, only the exoccipitals are present (Trueb, 1993; Duellman and Trueb, 1994). However, this reduction in elements does not only affect the occiput. The rest of the braincase is also affected and reduced in lissamphibians compared to other tetrapods (Figure 1.3; de Beer, 1937; Romer and Witter, 1942; Romer, 1946; Romer and Parsons, 1977; Duellman and Trueb, 1994; Schoch, 1999; Schoch and Milner, 2014; Maddin, 2015).

In tetrapods, the entire braincase may consist of up to seven bones, including the parasphenoid (a membrane bone tightly integrated with the braincase), the sphenethmoid, the basisphenoid, the prootics, the opisthotics, the exoccipitals (occiput) and the basioccipital (occiput), and then an eighth element is the supraoccipital which contributes to the dorsal portion of the occiput (Figure 1.3; de Beer, 1937; Romer and Witter, 1942; Romer, 1946; Romer and Parsons, 1977; Duellman and Trueb, 1994; Schoch, 1999; Schoch and Milner, 2014; Maddin, 2015). In extant amphibians, only the parasphenoid, a reduced sphenethmoid, the otic elements (prootics and opisthotics), and paired exoccipitals are present (Figure 1.3; de Beer, 1937; Romer, 1946; Romer and Parsons, 1977; Trueb, 1993; Duellman and Trueb, 1994; Schoch, 1999; Schoch and Milner, 2004; Maddin, 2015). While other researchers (e.g., de Beer, 1937) have suggested that the reduced elements in the braincase in lissamphibians are secondarily derived, this hypothesis has not been 
formally tested with appropriate characters and ancestral character state reconstruction methods. Furthermore, braincase morphology in general is known to have a stronger phylogenetic signal than other regions of the head and axial skeleton (e.g., Cardini and Elton, 2008; Goswami and Polly, 2010; Maddin et al., 2012b; Brazeau and de Winter, 2015; Maddin, 2015). Thus, any phylogeny that attempts to reconstruct the ancestral state for the lissamphibian occiput benefits from including characters relevant to all braincase elements, in addition to the occiput.

The relatively simplified complement of bones contributing to the occiput in extant lissamphibians has been hypothesized by palaeontologists to be the result of heterochronic events where various kinds of paedomorphosis, such as miniaturization and truncated development, has resulted in fewer bones in the skull, and thus the occiput (e.g., Bolt, 1969; 1977; Milner, 1988; Boy and Sues, 2000; Schoch, 2013a). However, since the correspondence between specific occipital ossifications and somitic contribution to the occiput has not been established outside of a few model organisms, a non-paedomorphosis mechanism (such as reduced number of occipital somites) for the reduced occiput of lissamphibians remains a possibility. 
A
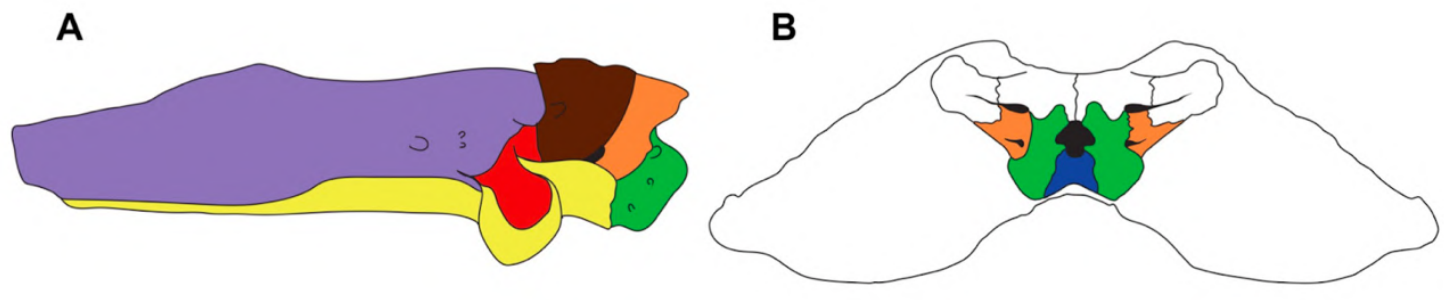

C

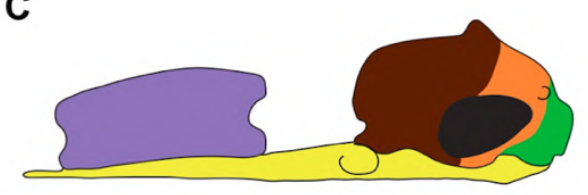

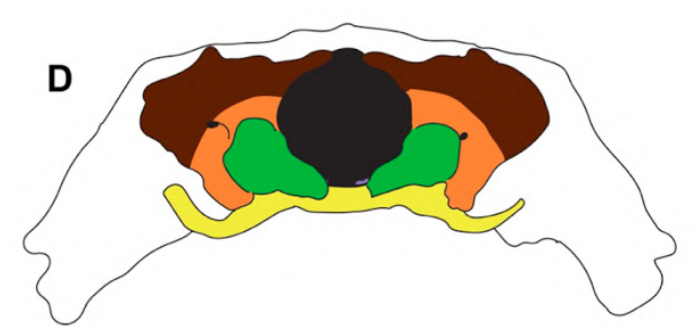

Figure 1.3. A comparison of the braincase and occiput of extinct and extant tetrapods reveals the relatively reduced condition of these parts of the skull in lissamphibians in comparison to those of other early tetrapods. A) and B) The extinct tetrapod Eryops in lateral (A) and occipital (B) view, showing a nearly full complement of ossifications with seven bones present. C) and D) The extant salamander Hynobius in lateral (C) and occipital (D) views, showing the reduced complement of ossifications with only five bones present. The sphenethmoid is purple, the basioccipital is blue, the basisphenoid is red, the exoccipitals are green, the parasphenoid is yellow, the opisthotic is orange and the prootic is brown. Not to scale.

One potential osteological correlate has been proposed for such investigations: foramina pertaining to the posterior-most cranial nerve, cranial nerve twelve (n.XII; Maddin et al., 2020), which is also generally referred to as the hypoglossal nerve or hypoglossal nerve complex in amniotes (de Beer, 1937; Romer and Edinger, 1942; Romer and Parsons, 1977; Grande and Bemis, 1998). As the most posteriorly situated cranial nerve, the hypoglossal nerve marks the boundary between cranial and spinal nerves (Barnard, 1940; Noden, 1983; Couly et al., 1993; Ferguson and Graham, 2004; Yaryhin and Werneburg, 2018). The hypoglossal nerve can be differentiated from the spinal nerves by its lack of dorsal roots (Hunter, 1935; Brodal, 2010). In contrast, true spinal nerves (i.e., those that emerge from the spinal cord) always possess well developed dorsal roots (Hunter, 1935; Brodal, 2010). The branches of the hypoglossal nerve emerge from the 
central nervous system in a one-to-one registry with respect to the somites, similarly to the more posterior spinal nerves (Mall, 1891; Romer and Parsons, 1977; Ferguson and Graham, 2004; Kardong, 2015). In amniotes, such as chicken, each branch of the hypoglossal nerve is associated with the anterior portion of an occipital somite (Hazelton, 1970; Noden, 1983; Ferguson and Graham, 2004), and, as such, emerge through the occipital bones of the skull in adults, specifically the exoccipital (Figure 1.4A).

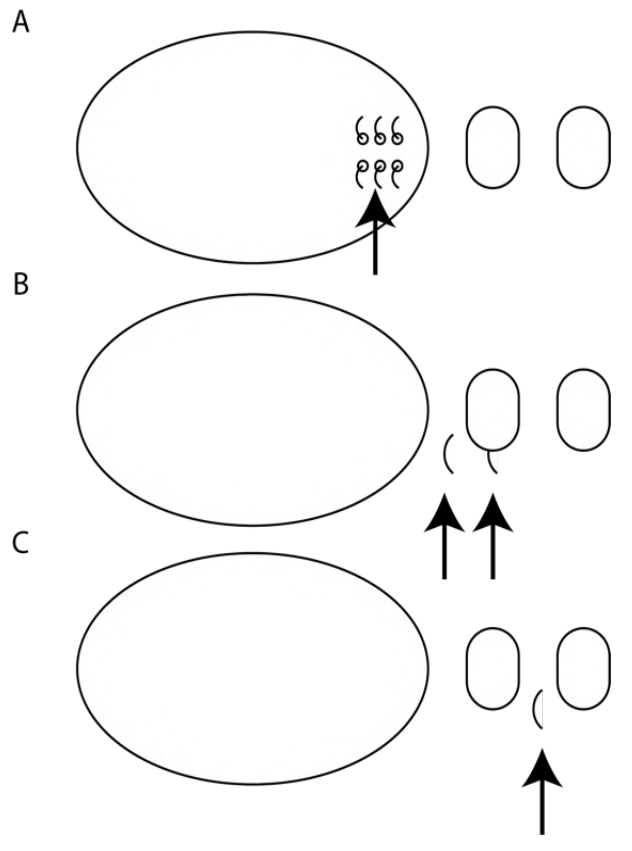

Figure 1.4. Schematic synthesized from the literature showing where the hypoglossal (XII) nerve emerges with respect to the brain or spinal column. Anterior is to the left and posterior to the right. A) In amniotes, like the chicken, the hypoglossal nerve (arrows) emerges from the skull via several foramina in the occiput. B) In salamanders the hypoglossal passes laterally between the occiput and the first vertebral element or via a foramen in the first vertebral element. C) In frogs the hypoglossal passes laterally between the first and second vertebral elements. These morphologies have not been confirmed in Xenopus laevis nor in Ambystoma mexicanum. Schematic synthesis based on Wake and Lawson (1973); Stuesse et al. (1983); Hüppi et al. (2019); Maddin et al. (2020).

In amphibians, the hypoglossal forms from what are typically referred to as the first or first and second spinal nerves, despite lacking dorsal roots diagnostic of true spinal nerves (e.g., Platt, 1897; Elliot, 1907; Norris, 1908; Norris, 1913; Barnard, 1940; Herrick, 1948; Wake and Lawson, 1973; Stuesse et al., 1983; Roth et al., 1984; Wake, 1992; 1993; Naumann and Olsson, 2018). Each branch of the hypoglossal nerve is at most associated with the last occipital somite and up to the first two vertebral somites (Wake, 1993; Detwiler, 1929; Edgeworth, 1935). In salamanders, the branches of the hypoglossal nerve 
extends laterally from the spinal cord between the occiput and the first vertebra, or via a foramen on the first vertebral element (Figure 1.4B; Platt, 1897; Norris, 1908; Norris, 1913; Herrick, 1948; Wake and Lawson, 1973; Roth et al., 1984). In frogs, the branches of the hypoglossal extend laterally between the first and second vertebrae, where it is referred to as the second spinal nerve (Figure 1.4C; Elliot, 1907; Barnard, 1940; Stuesse et al., 1983; Naumann and Olsson, 2018). Therefore, in salamanders the hypoglossal appears to be associated with the first vertebral somite, and in frogs, with the second vertebral somite, and not with any of the occipital somites.

The one-to-one registry of hypoglossal branches and somites is a tempting tool to use in determining somite composition of the occiput when developmental data are lacking. However, in amniotes, a variable number of hypoglossal branches emerges from the skull via a variable number of foramina (e.g., Barnard, 1940; Noden, 1983; Couly et al., 1993; Ferguson and Graham, 2004; Yaryhin and Werneburg, 2018), and no hypoglossal foramina are present in amphibians (Estes, 1965), as the hypoglossal nerve emerges posterior to the skull. However, in a recent review, Maddin et al. (2020) synthesized historical and recent accounts of occipital development in many tetrapods. Together, these data revealed that a much less variable and phylogenetically significant pattern of skull-neck boundary location exists among tetrapods. Three conditions for tetrapods were identified: (1) the amniote condition, with a skull-neck boundary location at the posterior limit of the hypoglossal nerve complex, (2) the condition in salamanders and caecilians, with a skullneck boundary location within the anterior part of the hypoglossal nerve complex, and (3) the condition in frogs with a skull-neck boundary location anterior to the hypoglossal nerve complex (Maddin et al., 2020). Under this organizational regime, the total number of 
occipital somites remains somewhat variable, but in general amniotes incorporate more somites into their occiput than salamanders and caecilians, which in turn incorporate more occipital somites than frogs. By including fossil taxa and using the presence or absence of hypoglossal nerve foramina, the hypothesis that extant lissamphibians represent the ancestral tetrapod condition (e.g., Couly et al., 1993; Ferguson and Graham, 2004) is rejected and the evolutionary pattern proposed by de Beer (1937) and others (Romer and Edinger, 1942; Estes, 1965; Clack and Holmes, 1988) in which lissamphibians are instead secondarily reduced is supported (Maddin et al., 2020). Therefore, a growing body of evidence supports the amniote-like condition as the likely ancestral condition for tetrapods (Maddin et al., 2020). This would mean that instead of incorporating more somites in the occipital region during the evolution of amniotes (i.e., a posterior shift in the skull-neck boundary), amphibians actually underwent a reduction in the number of somites being incorporated into their occiput during their evolution (i.e., an anterior shift of the skullneck boundary). The mechanism(s) underlying this event remain unknown.

\section{3: The developmental mechanisms underlying axial patterning}

It is now well understood that patterns of Hox gene expression determine the fate of the segments of the anterior-posterior axis in many organisms, including vertebrates (e.g., Gaunt et al., 1988; Ruiz i Altaba and Jessell, 1991; Ramírez-Solis et al., 1993; Krumlauf, 1994; Burke et al., 1995; Pearson et al., 2005; Handrigan and Wassersug, 2007). Hox genes are examples of a group of genes known as homeobox genes that share a similar portion of sequence, approximately 180 base pairs in length, called the homeobox (McGinnis and Krumlauf, 1992). Vertebrate Hox genes are organized into four clusters (A, B, C, and D) and thirteen paralogous groups (1-13; Krumlauf, 1994; Pearson et al., 2005; 
Mallo et al., 2010). Each paralogous group consists of the genes within each cluster (e.g., paralog group nine: hoxa9, hoxb9, hoxc9, hoxd9) and these genes have shared expression properties. Hox genes exhibit colinear expression, where they are expressed along the anterior-posterior axis in the same order they appear on their chromosome; the most 3' genes have a more anterior expression limit along the anterior-posterior axis than the genes that are located closer to 5' end of the chromosome (McGinnis and Krumlauf, 1992; Krumlauf, 1994). Therefore, genes like hoxb4 have a more anterior expression domain than hoxb7 along the anterior-posterior axis. Colinear expression also means that Hox genes that are from the same paralogous group have similar expression domains (Manzanares et al., 2001). In addition to similar expression domains, Hox genes also have nested expression domains, such that more Hox genes are expressed in the posterior region than the anterior region (Mallo et al., 2010). Hox genes also exhibit posterior dominance, where knocking out anterior Hox genes does not always change the fate of posterior Hox genes as they can compensate for the missing anterior Hox gene (Mallo et al., 2010).

In amniotes, the anterior limit of specific Hox genes has been shown to mark key morphological changes along the anterior-posterior axis (Figure 1.5; Burke et al., 1995). For example, even though chickens and mice have different numbers of somites that contribute to their cervical vertebrae, in both the anterior limit of expression for hoxc6 marks the transition between cervical and thoracic vertebrae (Figure 1.5; Burke et al., 1995). The pattern of expression for Hox genes, i.e., which Hox genes are expressed where along the anterior-posterior axis, gives each segment their identity, i.e., cervical, thoracic, lumbar, etc. (Ramírez-Solis et al., 1993; Wellik and Capecchi, 2003; Nowicki and Burke, 2000; Mallo et al., 2010). 


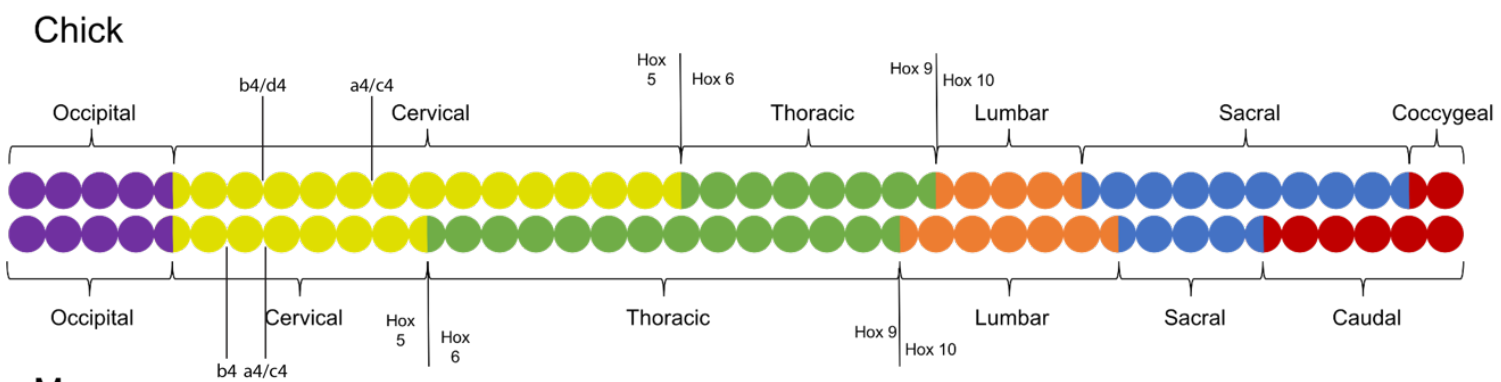

Mouse

Figure 1.5. Schematic of axial somites, their fate and associated Hox gene expression patterns in chicken (top) and mouse (below), with anterior to the left and posterior to the right. Each colour represents a different type of axial structure (e.g., cervical or thoracic vertebrae), and the location of Hox genes that are responsible for determining those fates mark each transition. Modified from Burke et al. (1995).

The role Hox genes play in patterning the segments of an embryo has been shown in studies based on Hox gene mutants, where particular embryonic segments were transformed into the identity of other segments (e.g., Kessel et al., 1990; Ramírez-Solis et al., 1993; Horan et al., 1995; Scaal, 2016) - i.e., homeotic transformations. Experiments in mice showed that hoxd3 knockdown mutants exhibit fusion of the first vertebra (the atlas) to the occiput (Scaal, 2016). Other studies involving Hox gene mutants or loss of function experiments have transformed cervical vertebrae to thoracic identities (RamírezSolis et al., 1993), produced additional cervical vertebrae (Kessel et al., 1990), or removed vertebrae (Horan et al., 1995).

Similar mutations have also been demonstrated using retinoic acid, a known chemical that influences Hox gene expression domains, to manipulate the location of Hox expression (Durston et al., 1989; Sive et al., 1990; Ruiz i Altaba and Jessel, 1991). Many genes that are expressed during early development, including Hox genes, are sensitive to retinoic acid. Hox genes are activated in developing embryos by endogenous retinoic acid and applying exogenous retinoic acid to embryos during early development can transform 
the fate of developing neural ectoderm and embryonic mesoderm (e.g., Durston et al., 1989; Ruiz i Altaba and Jessel, 1991; Marshall et al., 1994; Kolm and Sive, 1995). Higher concentrations of retinoic acid in the developing embryo causes anterior shifts in Hox gene expression, resulting in segments transforming into structures of a more posterior fate than normal (Figure 1.6B; Durston et al., 1989; Sive et al., 1990; Ruiz i Altaba and Jessel, 1991). Similarly, inhibiting retinoic acid can also transform the fate of developing embryonic segments (Schuh et al., 1993). Inhibiting retinoic acid shifts Hox gene expression posteriorly and causes segments to transform into a more anterior fate than normal (Figure 1.6C; Schuh et al., 1993; Kronmiller et al., 1995; Di Renzo et al., 2007).

A

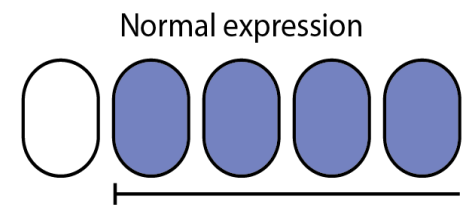

B

Overexpression of retinoic acid

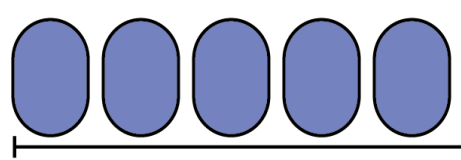

C Inhibition of retinoic acid

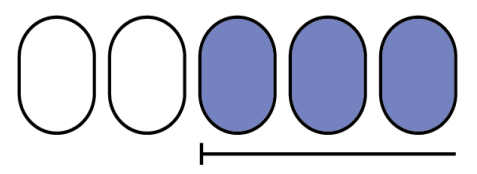

Figure 1.6. Retinoic acid causes homeotic transformations along the anterior (left) to posterior (right) axis. A) The fate of vertebral elements (purple) are shown in their normal location along the axis, with the anterior limit of Hox gene expression (black line). B) Retinoic acid overexpression in the developing embryo causes Hox genes to express more anteriorly than normal and the identity of each element to be shifted posteriorly. The identity of the first segment in B has changed to the identity of the second segment in A. C) Retinoic acid inhibition in the developing embryo causes Hox genes to express more posteriorly than normal the identity of each element to be shifted anteriorly. The identity of the third segment in $\mathrm{C}$ has changed to the identity of the second segment in $\mathrm{A}$.

Similar studies of Hox gene expression domains that then examined the resulting internal (i.e., skull) morphology have not been conducted in amphibians. It is presumed, due to deep homology of Hox genes and Hox function across vertebrates, that homologous Hox genes are expressed in a similar pattern to that observed in amniotes. However, this must be confirmed in order to expand the comparative basis of axial patterning, particularly 
as it may relate to skull-neck boundary evolution. Given the data collected in amniotes (e.g., Gaunt et al., 1988; Godsave et al., 1994; Burke et al., 1995; Manzanares et al., 2001; Gaufo et al., 2003), hox3 paralogs (occipital region expression) and hox4 paralogs (anteriormost cervical region expression) are of special interest in the story of skull-neck boundary evolution.

\section{4: Project goals and thesis outline}

The goal of this thesis is to elucidate some of the above-mentioned, outstanding aspects of the morphology and development of the skull-neck boundary in lissamphibians, such that events in the evolution of the boundary may be better understood in lissamphibians. As such, I have two hypotheses that I wish to test. The first hypothesis I wish to test posits that the reduced condition of the amphibian occiput is secondarily derived and not the ancestral condition for tetrapods. The second hypothesis posits that perturbing Hox gene expression domains can cause homeotic transformations at the occiput in A. mexicanum and X. laevis. In order to address these hypotheses, I will explore aspects of the lissamphibian skull-neck boundary from phylogenetic, morphological, and developmental aspects in three data chapters.

In Chapter 2, I investigate my first hypothesis, that the extant lissamphibian condition of the occiput is secondarily derived. To do this, I produce a high-resolution phylogeny for extant lissamphibian origins that includes new characters that target the condition of the occiput and braincase in extinct and extant taxa. Previously published phylogenies for lissamphibians and their fossil relatives were taxonomically limited, did not include both fossil and extant amphibians, or were missing recently discovered, taxonomically informative fossils (e.g., Schoch and Rubidge, 2005; Frost et al., 2006; 
Anderson et al., 2008a; 2008b; Fröbisch and Schoch, 2009; Bourget and Anderson, 2011; Maddin, et al., 2012a; 2012b; Schoch, 2012; Pyron and Wiens, 2013). Furthermore, while molecular evidence supports the monophyly of lissamphibians, their origin and relationship to extinct tetrapods has been contested (e.g., Marjanovic and Laurin, 2007; Ruta and Coates, 2007), and so frequent updates are required to improve confidence in patterns of relationships. Finally, many previously published matrices included few braincase and/or occiput characters, particularly characters that might reveal clues about occipital somite contribution.

To address these shortcomings in previously available matrices, I build upon a recently published Dissorophoidea matrix (Schoch, 2018) adding in key braincase characters (including occiput characters) and coding twelve additional, relevant taxa. My revised matrix is analyzed by applying maximum parsimony inference and Bayesian inference. The resulting tree allows me to reconstruct the ancestral character states for braincase and occiput morphology in the lineage leading to lissamphibians. Parsimonybased and maximum likelihood-based ancestral character state reconstruction methods were completed to reveal events in the evolution of the lissamphibian braincase. I conclude by providing evidence to support the hypothesis that the extant lissamphibian condition of the occiput is secondarily derived.

In Chapter 3, I investigate the normal development of the skull, focusing on the occiput, in the two extant lissamphibian model species, A. mexicanum for salamanders and $X$. laevis for frogs, in order to establish a developmental and morphological baseline for comparisons needed to test my second hypothesis (Chapter 4). First, to confirm the number of occipital somites in the skull of the two species and the structures they go on to 
contribute to, I conduct fate mapping studies. Modelling my own protocols after those of Piekarski and Olsson (2007), I repeat the occipital somite fate map for A. mexicanum and generate the first occipital somite fate map for $X$. laevis embryos by micro-injecting dextran-fluorescein to track cell migration and differentiation. I then thin-section these embryos and determined precisely which somites contributed to the occiput, and to what skeletal structures, in both species.

With confirmed somitic origins of the occiput in hand, I then present a whole-mount stained developmental series of cartilage and bone for the skull of both species to document normal occiput (and skull) morphological development in detail. For both A. mexicanum and X. laevis, a staging table that documents the progression of normal skull development was not available. Thus, the morphology and timing of normal development of occipital structures could not previously be ascertained, which is needed for making comparisons with experimental animals in Chapter 4.

Finally, I present the stained nerves of A. mexicanum and X. laevis in order to examine and describe the gross morphology of the hypoglossal nerve as it relates to skullneck boundary structures in both species. This permits me to potentially use the position of the hypoglossal nerve relative to skull-neck boundary structures as an additional locus of comparison with experimental animals generated to address my second hypothesis (Chapter 4). Together, these three anatomical datasets add to the body of literature on the gross morphology of lissamphibians and reveal subtle differences in the occipital morphology and development between salamanders and frogs. By describing development in these two lissamphibian model organisms, I also provide details of what the reduced 
occipital morphology looks like in lissamphibians that can then be compared to other lissamphibians taxa in future studies.

In Chapter 4, I investigate my second hypothesis that perturbing Hox gene expression domains can cause homeotic transformations at the occiput in A. mexicanum and X. laevis. Previous research in amniotes such as chicken and mouse (e.g., Kessel et al., 1990; Ramírez-Solis et al., 1993; Burke et al., 1995; Horan et al., 1995; Scaal, 2016) have shown that the Hox paralogs expressed in somites at and around the skull-neck boundary are paralogs one through four. Hox paralogs one through three are found in the occipital region, whereas Hox paralog four is expressed in the anterior-most portion of the neck. Similar studies have not been conducted at all in A. mexicanum, whereas a study on the effect of exogenous retinoic acid on hoxb3 gene expression in X. laevis has been published (Godsave et al., 1998). However, in this latter study, these researchers did not examine the effects of retinoic acid on the developing skull, nor did they conduct retinoic inhibition morphology.

Thus, I conduct experiments exposing A. mexicanum and $X$. laevis embryos to either doses of exogenous retinoic acid or doses of retinoic acid inhibitors (i.e., citral), and examined the resulting morphology. The consequences of these experiments are then shown to be changes in the fate of segments along the anterior-posterior axis, wherein additional anterior vertebral elements appear when embryos are exposed to retinoic acid (representative of an anterior shift in somite fate) and vertebral segments are recruited into the head when embryos are exposed to citral (representative of a posterior shift in somite fate). These experiments suggest that changing Hox gene expression domains is capable of inducing homeotic transformations of occipital somites, and that these changes are 
potential drivers underlying the anterior shift of the evolution of the lissamphibian skullneck boundary. I confirm that homeotic transformations have occurred in these experimental specimens using cell-lineage tracing methods and the position of the hypoglossal nerve complex along the anterior-posterior axis.

Finally, in Chapter 5, I summarize the results of this thesis as they relate to an improved understanding of lissamphibian, and by extension tetrapod, skull-neck boundary evolution. I explore possibilities for why these Hox gene expression domains may have changed during lissamphibian evolution. This $\mathrm{PhD}$ thesis helps to elucidate the relationship between morphological and the developmental processes underlying a fundamental aspect of skull evolution. These are the first empirical analyses of skull-neck boundary morphology and development that reveal the potential of this work to re-write our understanding of skull evolution as we reveal how the amphibian condition was secondarily achieved, rather than perpetuate the inaccurate perception that they represent the ancestral condition for tetrapods, as currently thought. 


\section{Chapter 2: Braincase and occipital simplification and the origin of lissamphibians}

\section{1: Introduction}

The distinctive morphology of extant lissamphibians (frogs, salamanders, and caecilians) has been a leading cause in the lack of resolution of both their relationships to one another, and from which group of extinct tetrapods they are derived. Different phylogenetic analyses have placed them entirely within Temnospondyli (e.g., Figure 2.1A; Bolt, 1969; Rage and Janvier, 1982; Milner, 1988; Trueb and Cloutier, 1991; Milner, 1993; Schoch and Milner, 2004; Schoch, 2018), entirely within Lepospondyli (e.g., Figure 2.1B; Laurin and Reisz, 1997; Vallin and Laurin, 2004; Marjanović and Laurin, 2013), a group that may be polyphyletic (e.g., Pardo et al., 2017), or a combination thereof, where Batrachia (frogs and salamanders) reside within Temnospondyli and Apoda (caecilians) reside within Lepospondyli (e.g., Figure 2.1C; Lee and Anderson, 2006; Carroll, 2007; Anderson et al., 2008a; Skutschas and Martin, 2011). Despite all three hypotheses, and even a recent fourth hypothesis that recovers Batrachia within Dissorophoidea and Apoda within Stereospondyli (Pardo et al., 2017), maintaining a presence in the literature, there has been a growing consensus that lissamphibians are a monophyletic assemblage derived from within Temnospondyli (e.g., Estes, 1965; Trueb and Cloutier, 1991; Ruta and Coates, 2007; Fröbisch and Schoch, 2009a; Sigurdsen and Bolt, 2010; Maddin et al., 2012a; Schoch, 2018), and more specifically from within the amphibamid dissorophoids (e.g., Bolt, 1977; 1979; Milner, 1988; Schoch and Milner, 2004; Maddin et al., 2012a; Schoch, 2018). 

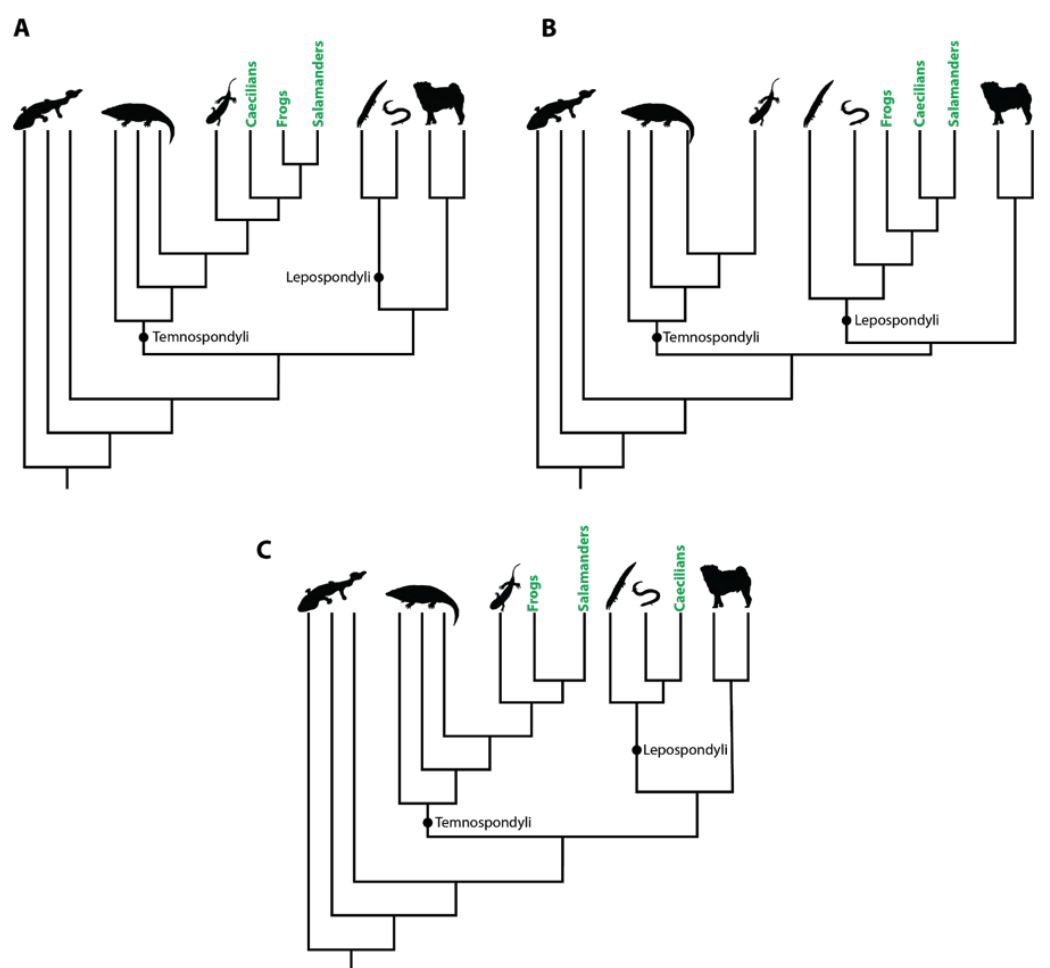

Figure 2.1. The three hypotheses for extant lissamphibians (green text) and their closest relatives. A) A monophyletic Lissamphibia is found within Temnospondyli (e.g., Milner, 1993; Schoch and Milner, 2004; Schoch, 2018). B) A monophyletic Lissamphibia is found within Lepospondyli (e.g., Marjanović and Laurin, 2013). C) Lissamphibia is paraphyletic with Batrachia found within Temnospondyli and caecilians found within Lepospondyli (e.g., Anderson et al., 2008a; Skutschas and Martin, 2011). Silhouettes are from open source PhyloPic.org.

The phylogenetic framework provided by this growing confidence in a monophyletic Lissamphibia derived from within Temnospondyli permits investigations of patterns of morphological evolution of lissamphibians, including the origin of their highly derived form. A combination of new discoveries (e.g., Holmes et al., 2013; Pardo et al., 2017; Gee and Reisz, 2018) as well as extensive reanalysis of existing material (e.g., Maddin et al., 2012a; 2012b; 2013; Schoch and Sues, 2013; Ascarrunz et al., 2016; Gee et al., 2017; Schoch, 2017; Gee and Reisz, 2018; Pérez-Ben et al., 2018) provide the foundation for potentially high-resolution investigations of morphological evolution with great numbers of taxa and forms. Unfortunately, many currently available phylogenetic 
analyses take place at either very broad taxonomic levels (e.g., Schoch and Rubidge, 2005; Anderson et al., 2008a; Maddin et al., 2012a; Schoch, 2013b), or very fine, taxonomically exclusive levels (e.g., Anderson et al., 2008b; Fröbisch and Schoch, 2009a; Bourget and Anderson, 2011). In the case of the former, detailed variation is glossed over by the pruning of many taxa to cover a broader sampling; whereas with the latter, the distribution of traits at higher clade levels is missed due to entire clades being excluded.

One such series of evolutionary events concerns the origin of the relatively simple condition of the braincase, which includes the bones of the occiput, in terms of the number of ossifications and the extent of ossification of individual bones in lissamphibians in comparison to that of other temnospondyls and amniotes. Extinct temnospondyls (e.g., Edops, Eryops) and amniotes have numerous ossifications making up the braincase, including the parasphenoid, sphenethmoid, basisphenoid, prootics, opisthotics, exoccipitals, basioccipital and occasionally a supraoccipital (Figure 1.3; de Beer, 1937; Romer and Witter, 1942; Romer, 1946; Romer and Parsons, 1977; Duellman and Trueb, 1994; Schoch, 1999; Schoch and Milner, 2014; Brusatte et al., 2015; Maddin, 2015; Schoch, 2018). The parasphenoid is an unpaired, membranous or dermal bone (Figure 1.3, yellow; de Beer, 1937; Atkins and Franz-Odendaal, 2016). The other bones that commonly contribute to the braincase in tetrapods (i.e., sphenethmoid, basisphenoid, prootics, opisthotics, exoccipitals, basioccipital, and supraoccipital) are endochondral bones of the chondrocranium (Figure 1.3; de Beer, 1937).

The presence and degree of ossification of these eight braincase elements (four of which are occipital) varies among temnospondyl taxa, with basal members such as Edops having a heavily ossified braincase compared to more derived members such as Eryops 
(Figure 2.2; Romer and Witter, 1942; Schoch and Milner, 2014). Both stereospondyls and dissorophoids, two temnospondyl lineages, vary in the number and degree of ossifications in the braincase (Figure 2.2). Many more later-diverging stereospondyls lack an ossified basioccipital (e.g., Figure 2.2; Gerrothorax [Witzmann et al., 2012]) or possess a weakly ossified basisphenoid (e.g., Mastodonsaurus [Schoch, 2002]) when compared to earlydiverging stereospondyls (e.g., Archegosaurus [Witzmann, 2006]). In the dissorophoid lineage, braincase ossification may also be reduced, such as in branchiosaurids, where the basisphenoid is incompletely ossified (Boy, 1972).

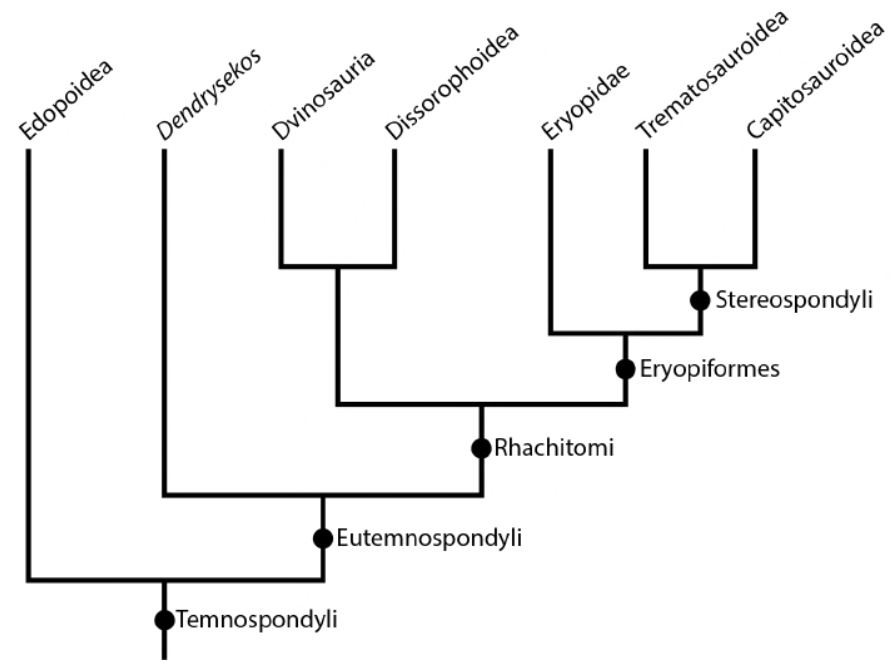

Figure 2.2. A simplified phylogenetic tree for Temnospondyli showing the broad level groups discussed in this chapter. Modified from Schoch (2013b).

Extant lissamphibians have a relatively simple braincase compared to all other temnospondyls in terms of the number of ossifications and even in terms of their chondrocranial precursors. Extant lissamphibians possess only the parasphenoid, a sphenethmoid, the otic elements (i.e., prootic, opisthotic) and paired exoccipitals as discrete ossifications (Figure 1.3; de Beer, 1937; Romer, 1946; Romer and Parsons, 1977; Duellman and Trueb, 1994; Schoch, 1999; Schoch and Milner, 2004; Maddin, 2015). In 
the case of the basisphenoid and basioccipital, the cartilaginous precursors are considered absent from the chondrocranium of extant lissamphibians (de Beer, 1937; Duellman and Trueb, 1994; Müller, 2006; Maddin, 2015; Schoch, 2018). Additionally, other braincase bones that are commonly found in temnospondyls (e.g., sphenethmoid [de Beer, 1937; Maddin, 2015]) are comparatively reduced in extant lissamphibians (e.g., Altig, 1969; Trueb et al., 2000; Maddin, 2015) whereas other elements that are only occasionally found in temnospondyls, but are commonly present in amniotes (e.g., supraoccipital [Berman, 2000; Brusatte et al., 2015]), are absent in extant lissamphibians (Figure 1.3; Schoch and Milner, 2004; Müller, 2006; Schoch, 2018). In the case of the supraoccipital, caecilian lissamphibians further lack its cartilage precursor, the tectum synoticum (de Beer, 1937; Müller, 2006).

The simple condition of the lissamphibian braincase has historically led some authors, working on extant taxa alone, to hypothesize it is the product of the ancestral developmental pattern for tetrapods (e.g., see Figure 1.2A; Augier, 1931; Couly et al., 1993; Ferguson and Graham, 2004). However, other authors whose work included fossil taxa have instead hypothesized that the lissamphibian condition represents a derived condition (e.g., see Figure 1.2B; de Beer, 1937; Romer and Edinger, 1942; Clack and Milner, 2009). However, without a test of character evolution and ancestral character state reconstruction, the timing and nature of morphological evolutionary events leading to the origin of the simple lissamphibian condition remain speculative.

The present study attempts to reveal the evolution of the lissamphibian braincase through a test of character evolution via the creation of new characters and the utilization of a broad taxonomic sample of Dissorophoidea. Here I built on the recently published 
Dissorophoidea matrix by Schoch (2018), sampling a total of 42 dissorophoid taxa (four from Micromelerpetidae, 17 from Olsoniformes, and 21 from Amphibamiformes). The original matrix of Schoch (2018) included 28 dissorophoid taxa (four from Micromelerpetidae, eight from Olsoniformes, and 16 from Amphibamiformes). My sampling is as inclusive as possible and represents an improvement in this regard to earlier analyses. For example, Schoch (2012) included 25 extinct dissorophoid taxa, and Fröbisch and Schoch (2009a) included 31 extinct dissorophoid taxa. Maddin et al. (2012a) is the only recent analysis to broadly sample both extinct and extant members of Lissamphibia, but even this matrix only included 27 dissorophoid taxa, of which ten were caecilian taxa. Three new characters pertaining to the braincase were created and scored for as many of these taxa as possible. Specifically, two new characters that correspond to aspects of the occiput that may shed light on the developmental state (i.e., potential somitic contribution). My analysis and the resulting trees allow me to reconstruct the ancestral character states of these braincase characters and present a hypothesis of braincase evolution in the lineage leading to Lissamphibia. I discuss these results as they relate to the origin of the lissamphibian form.

\section{2: Methods}

\subsection{1: Phylogenetic analyses}

I approached this problem from the perspective that lissamphibians are monophyletic dissorophoid temnospondyls, which is made up of two clades: the Olsoniformes, and the recently proposed Amphibamiformes (Amphibamidae plus Branchiosauridae [Schoch, 2018]). To produce a broadly sampled phylogeny for Dissorophoidea, I utilized the latest published matrix from Schoch (2018). There, one 
relevant braincase character - presence or absence of the basioccipital (character 93) - was included. I additionally added three more braincase characters to capture variation in the degree of braincase ossification and complexity (and chondrification; Appendix A). This includes: the presence or absence of the basisphenoid (109); the presence or absence of hypoglossal nerve foramina on the exoccipital (110); and the presence or absence of the sphenethmoid floor, which when absent results in small, paired sphenethmoids (111).

I made the decision to include as many taxa as possible in order to avoid subjective user bias in the exclusion of taxa that might actually be important. As such, twelve extinct taxa were also added to the matrix. The additions included members of some other temnospondyl clades to provide broader evolutionary context: Trimerorhachis, Sclerocephalus, Eryopidae, and Acanthostomatops. Eleven additional dissorophoid taxa were also added: Tambachia, Anconastes, Scapanops, the Rio Arriba taxon, Kamacops, Brevidorsum, Reiszerpeton, Aspidosaurus, Platyhystrix, Rubeostratilia, and Plemmyradytes. Additionally, three extant taxa were added to the matrix: representatives for each of the extant lissamphibian groups (Ascaphus for frogs, Hynobius for salamanders, and Rhinatrema for caecilians). The final matrix thus consisted of 48 taxa, including the outgroup taxon, Dendrysekos (= Dendrerpeton [Schoch and Milner, 2014]) and 111 characters (Appendix B). Taxa were coded for characters not present in their original matrix from the literature and some first-hand observations of specimens (see Appendix C for a full list of taxa and sources).

The final matrix was analyzed using maximum parsimony inference and Bayesian Inference. The parsimony analysis was run using PAUP v. 4.0a161 (Swofford, 2003) for MacIntosh. Dendrysekos was set as the outgroup for rooting. The heuristic search option 
and tree bisection-reconnection branch-swapping algorithm was used with 10,000 additional random sequence replicates selected. Multistate taxa were treated as polymorphic, and all characters were unordered and weighted equally. The Bayesian analysis was run in MrBayes version 3.1.2 (Huelsenbeck and Ronquist, 2001) using the Mk model and running a Markov chain Monte Carlo for 5 million generations, with a sampling frequency of 100 and a diagnostic frequency of 1,000. Resulting posterior probabilities were inspected for convergence in the program Tracer v1.6 (Rambaut et al., 2014) for MacIntosh.

\subsection{2: Ancestral character state reconstructions}

Ancestral character state reconstructions were performed on the resulting strict consensus tree from the parsimony analysis as well as on the All Compatible Clades consensus tree from the Bayesian analysis. For the strict consensus tree from the parsimony analysis, the ancestral character state reconstruction analysis was performed on the braincase characters (characters 93, 109, 110, and 111) using Mesquite version 3.10 build 765 (Massidon and Maddison, 2018). Ancestral character states were estimated using both parsimony and the maximum likelihood (Mk1) statistical model, the latter of which calculates the proportional likelihoods of each character state at ambiguous nodes. For the All Compatible Clades tree generated by the Bayesian analysis, the evolutionary scenarios for the braincase characters were again analysed with maximum likelihood ancestral reconstructions, with the assumption of equal rates of evolution. This analysis was completed in R (R Core Team, 2017) using ACE (ancestral character estimation) and phytools packages (Revell, 2012). This dual approach for ancestral character state reconstruction follows the currently widely accepted methodologies in the literature (e.g., 
Maddin et al., 2012b; Hsiang et al., 2015; Pereyra et al., 2016; Samuels et al., 2017; Sasson and Ryan, 2017; Kieren et al., 2018).

\section{3: Results}

\subsection{1: Phylogenetic analyses}

The maximum parsimony analysis resulted in 81,922 most parsimonious trees, each with 345 steps in length (consistency index $=0.3623$; retention index $=0.7287$ ). The strict consensus tree from the maximum parsimony analysis is overall poorly resolved at the finer taxonomic levels (Figure 2.3, right side), whereas the All Compatible Clades consensus tree from the Bayesian analysis has virtually no polytomies (Figure 2.3, left side). The broader family level divisions, and subgroupings within, are very similar between both analyses (Figure 2.3). For example, in both analyses, the Amphibamiformes form a clade that includes Amphibamidae, Branchiosauridae, and a monophyletic Lissamphibia. In both analyses caecilians and Eocaecilia are found to be the sister group of Batrachia; however, in the parsimony analysis the relationships within Batrachia are poorly resolved where Karaurus plus salamanders form an internal clade that occurs in a polytomy with frogs and Triadobatrachus. Gerobatrachus forms the sister taxon to Lissamphibia in the Bayesian analysis (Figure 2.3, left), whereas in the parsimony analysis Gerobatrachus, Georgenthalia, and Lissamphibia are recovered in a polytomy (Figure 2.3, right). The Dissorophidae and Trematopidae clades that together make up the Olsoniformes are recovered here, although the former is poorly resolved internally in the parsimony analysis. In both analyses the Olsoniformes is the sister clade to the Amphibamiformes (Figure 2.3). Also, in both analyses, Micromelerpetidae is found to be the sister group to the Olsoniformes plus Amphibamiformes clade, and thus represents the basalmost branch 
within Dissorophoidea. Perryella is the sister taxon to Dissorophoidea. One node below Perryella, Sclerocephalus and Eryopidae form a clade in both analyses, with Acanthostomatops on their stem. Finally, in both analyses, Trimerorhachis forms a polytomy with the outgroup Dendrysekos, at the base of the tree.

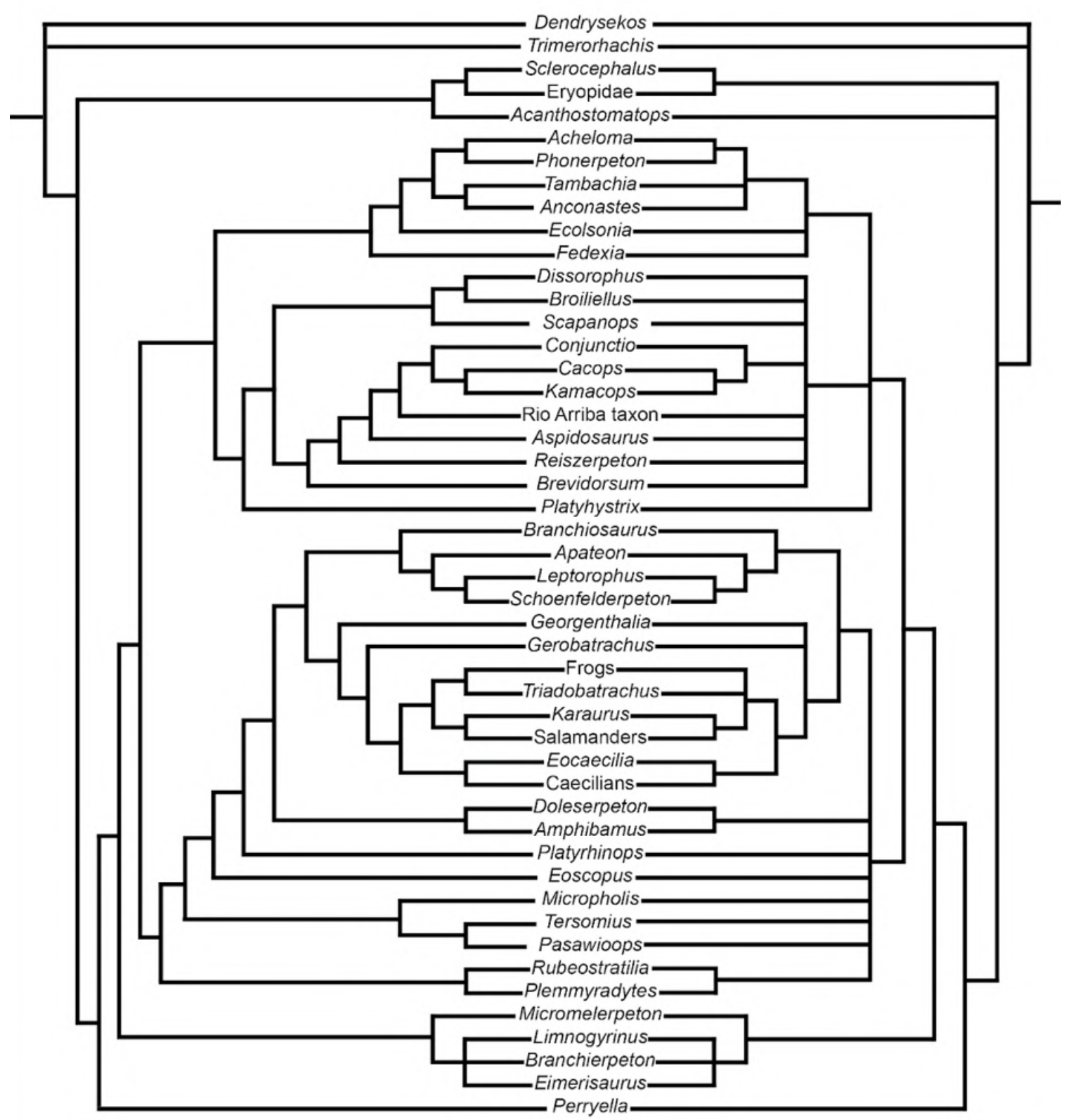

Figure 2.3. The results of the phylogenetic analyses of Dissorophoidea. The Bayesian analysis All Compatible Clades tree (left) obtained from MrBayes and the strict consensus tree (right) of 81,922 most parsimonious trees obtained from PAUP. 


\subsection{2: Ancestral character state reconstructions}

Ancestral character state reconstructions were conducted for the four most relevant braincase characters (characters 93, 109, 110 and 111) and were estimated using both parsimony and maximum likelihood on the results of the parsimony analysis, and maximum likelihood on the results of the Bayesian analysis. Schoch (2018) did include other braincase characters in his analysis, but they were regarding the morphology of the parasphenoid (Appendix A), and were thus not of particular relevance here. The results of all three analyses revealed the lineage leading to crown Lissamphibia is characterized by three absences that are identified to be losses and one ossification reduction that took place within the Amphibamiformes clade (Figure 2.4). The losses and the reduction were reconstructed at the same nodes using the maximum likelihood method on both the parsimony- and Bayesian-based trees; however, two of the losses and the reduction were reconstructed as ambiguous (i.e., reconstructed as occurring at one of several potential nodes) using parsimony methods of ancestral state reconstruction on the parsimony tree. I summarize the results for all three analyses below.

The parsimony reconstruction failed to resolve an unambiguous location for the reduction of the sphenethmoid to small, paired elements on the parsimony-based tree (Figure 2.4A). The zone of ambiguity for this character spans the nodes that include the higher amphibamiforms (Branchiosauridae, Georgenthalia, and Gerobatrachus; Figure 2.4A, Nodes C to D). The maximum likelihood reconstruction on the strict consensus tree recovered the sphenethmoid change at the base of Lissamphibia with a proportional likelihood of 0.96 (Figure 2.4, Node D). The same location was found for this character 
state with the maximum likelihood reconstruction on the Bayesian-derived tree with a likelihood value of 0.81 (Figure 2.4B, Node D).

The first loss is the complete loss of the basioccipital (i.e., of both the ossified element and the cartilaginous precursor), which occurs at the base of Amphibamiformes in all three analyses on both trees (Figure 2.4A; B, Node B). At least a partially ossified basioccipital (i.e., the condition found in branchiosaurids [Boy, 1972]) is retained in all other lineages studied here. The complete loss of the basioccipital at the base of Amphibamiformes was reconstructed as unambiguous using parsimony and with a proportional likelihood of 0.99 using maximum likelihood on the strict consensus tree from the parsimony analysis (Figure 2.4A, Node B). This is the same location reconstructed by maximum likelihood on the Bayesian tree with a likelihood value of 0.41 at the base of Amphibamiformes (Figure 2.4B, Node B), and with a likelihood value of 0.92 at the node that includes all Amphibamiformes except Rubeostratilia and Plemmyradytes (Figure 2.4B). 

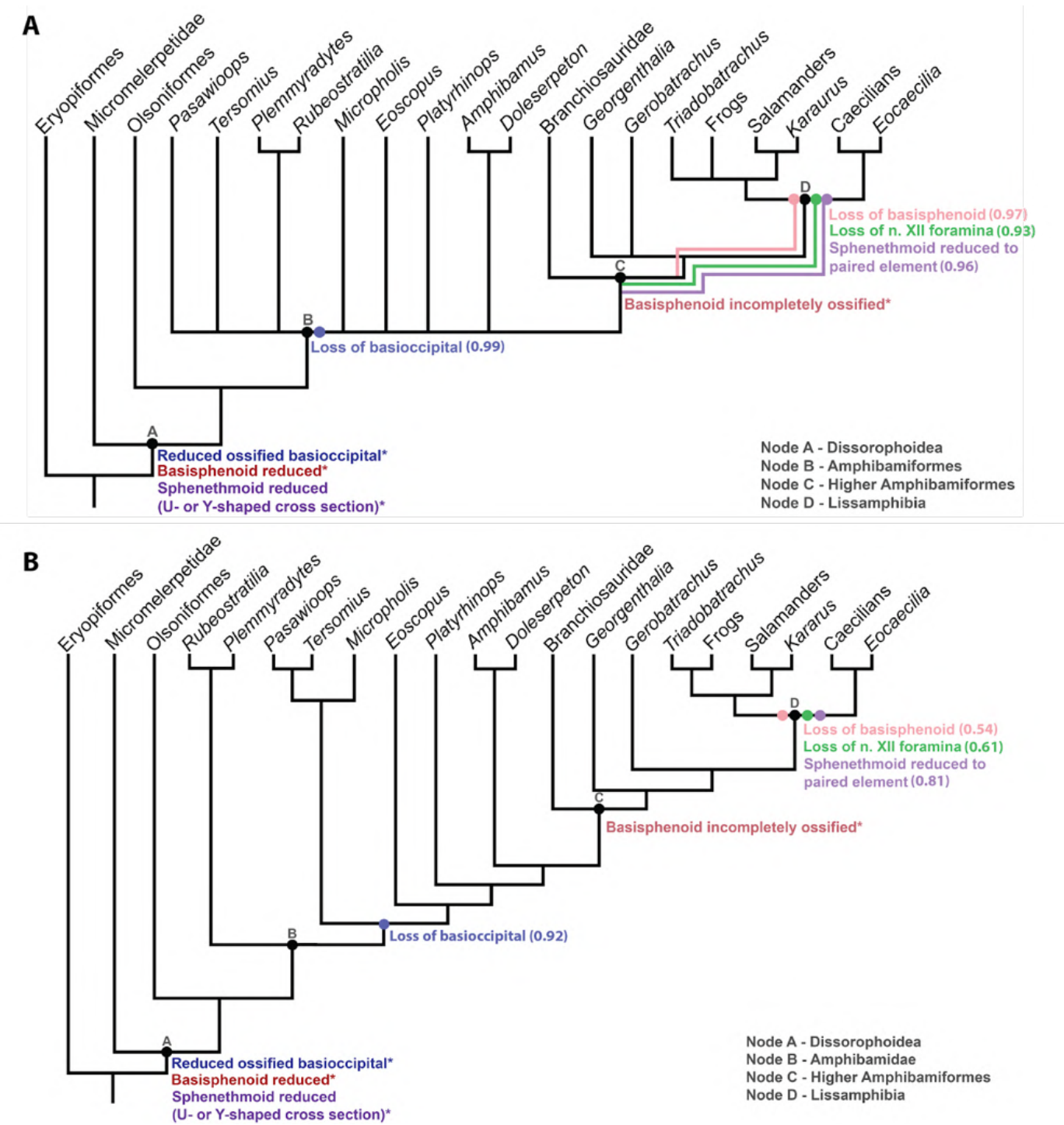

Figure 2.4. Summary of the ancestral character state reconstructions of the braincase characters on slightly pruned trees derived from both parsimony and Bayesian analyses. A) Parsimony and maximum likelihood ancestral character state reconstructions of the braincase characters on the strict consensus tree derived from the parsimony analysis. Coloured lines represent zones retrieved as ambiguous for the optimization of each character state under the parsimony criterion, and dots represent the locations retrieved as the most probable location for each character state based on the maximum likelihood method with its proportional likelihood value in parentheses. B) Maximum Likelihood ancestral character state reconstructions of the braincase characters on the All Compatible Clades consensus tree derived from the Bayesian analysis with associated likelihood value in parentheses. The discrete states of the four characters used in this analysis (93, basioccipital loss in blue; 109, basisphenoid loss in red; 110 hypoglossal nerve, n. XII, foramina lost in green; 111, sphenethmoid reduced to paired structures in purple) are described in the locations where states were optimized. Other reductions that are described in the text, but unable to be confirmed in fossil taxa (certain potential reductions) are indicated with asterisks. 
The second loss concerns the basisphenoid. The parsimony reconstruction failed to resolve an unambiguous location of the complete loss of the basisphenoid on the strict consensus tree from the parsimony analysis (Figure 2.4A, Node C to D). As a result, the zone of ambiguity spans the nodes that includes the branching of Georgenthalia, Gerobatrachus, and Lissamphibia. The maximum likelihood reconstruction on the strict consensus tree recovered the complete loss of the basisphenoid as occurring at the base of Lissamphibia with a proportional likelihood of 0.97 (Figure 2.4A, Node D). This is the same location obtained for the maximum likelihood reconstruction on the Bayesianderived tree with a likelihood value of 0.54 (Figure 2.4B, Node D).

The parsimony reconstruction again failed to resolve an unambiguous location for the location of the loss of foramina for the hypoglossal nerve (n.XII; Figure 2.4A). The zone of ambiguity for this character spans the nodes that include the higher amphibamiforms (Branchiosauridae, Georgenthalia, and Gerobatrachus; Figure 2.4A, Node $\mathrm{C}$ to D). The maximum likelihood reconstruction on the strict consensus tree recovered the loss of n.XII foramina at the base of Lissamphibia with a proportional likelihood of 0.93 . This is the same location of this character state change obtained for the maximum likelihood reconstruction on the Bayesian-derived tree with a likelihood value of 0.61 (Figure 2.4B, Node D).

\section{4: Discussion}

Here, I produce a revised, broadly sampled matrix that includes phylogenetically informative braincase characters for the inference of the intrarelationships of Dissorophoidea, and for the investigation of braincase evolution in the lineage leading to modern lissamphibians. My matrix samples from all dissorophoid clades and is inclusive 
of both the Olsoniformes and the Amphibamiformes lineages of Dissorophoidea. Additionally, representative members from early-diverging temnospondyl lineages have been included here for completeness and context (e.g., Trimerorhachis, Sclerocephalus, Eryopidae, and Acanthostomatops). Although the Bayesian Inference tree is well resolved, the parsimony tree reveals some problems may still exist with regards to resolving the relationships within the smaller clades of dissorophoids. With overall patterns of relationships largely congruent between analytical methods, it is hoped that this matrix, with its wide sampling of taxa and characters, provides a baseline for future analyses of this important group. As this analysis builds on other, previous phylogenetic analyses for lissamphibian origins, only a few key aspects of the resulting topology are noted here.

Overall, the addition of the braincase characters did not cause the topology of the phylogeny to change much relative to topologies found in other morphological analyses, such as those completed by Schoch (2018), which was the basis for this analysis. One difference in topology was the recovery of Gerobatrachus as the closest relative to Lissamphibia in my analysis, as opposed to its position as a stem batrachian recovered by Maddin et al. (2012a) and Anderson et al. (2008a). However, this position is identical to that found by Schoch (2018), where Gerobatrachus and Georgenthalia form a polytomy with Lissamphibia. Classically, Doleserpeton has been found as the most closely related amphibamid to Lissamphibia (e.g., Maddin et al. 2012a). In the current analysis, Gerobatrachus, Georgenthalia, and Branchiosauridae are all found as more closely related to Lissamphibia than Doleserpeton. Interestingly, the present analysis obtains Branchiosauridae nested within the group traditionally referred to as Amphibamidae (Figure 2.3). This relationship differs from the sister taxon relationship between 
Amphibamidae and Branchiosauridae, which has previously been found in other analyses (Schoch and Milner, 2008; Fröbisch and Schoch, 2009a; Maddin et al., 2012a; Schoch, 2013b; Pardo et al., 2017). However, see Schoch (2018) for a revised, more exclusive, definition of Amphibamidae that maintains its monophyly in this topological arrangement. Together Amphibamidae and Branchiosauridae form a group recently named Amphibamiformes (Schoch, 2018).

For the olsoniform portion of the tree, the traditional division of Olsoniformes into trematopids and dissorophids is found (Figure 2.3). Unlike in the Maddin et al. (2013) and Schoch (2012) analyses, where Platyhystrix is found as the sister taxon to the armored dissorophids (e.g., Dissorophus and Broiliellus), here Platyhystrix has a much more basal position as the sister taxon to the other Olsoniformes in the parsimony analysis. Interestingly, earlier analyses of Dissorophidae were better resolved than the present parsimony analysis. This suggests the inclusion of additional olsoniform and amphibamiform taxa is causing topology conflicts as adding only braincase characters to Schoch's (2018) matrix did not change the topology (tree not shown). Detailed work on braincase anatomy in these taxa, and the many poorly understood dissorophids that I chose to include, may help resolve these polytomies in future studies using parsimony. These issues, however, were not found in the Bayesian analysis, suggesting future study into various factors influencing the systematic approaches may also lead to improved resolution in a parsimony analysis.

In terms of braincase evolution, previous, qualitative interpretations of braincase transformations in the lissamphibian lineage led to the hypothesis that a series of secondary losses characterized the origin of the form of the lissamphibian braincase (de Beer, 1937; 
Romer and Edinger, 1942; Clack and Milner, 2009; Maddin, 2015). This evolutionary pattern, in addition to the presumed complete absence of a supraoccipital in the temnospondyl lineage that was generally accepted at the time but has since been revised with new data (e.g., Brusatte et al., 2015; Schoch, 2018), was suggested to be consistent with an amphibamid temnospondyl origin of Lissamphibia (Maddin, 2015). However, the braincase loss characters were never actually added to a matrix, and thus, their distribution had not been rigorously tested in an analysis of all of the characters. The quantitative analyses performed here has, for the first time, demonstrated that the lissamphibian braincase is the product of a stepwise sequence of losses and reductions, confirming it is not the ancestral condition for tetrapods and is indeed secondarily derived in its reduced state (de Beer, 1937; Romer and Edinger, 1942; Clack and Holmes, 1988; Maddin, 2015).

I propose the following stepwise sequence of loss events in the origin of the form of the lissamphibian braincase. Occurring in the anterior portion of the braincase is the transformation of the sphenethmoid from a large, single median element with a $\mathrm{U}$ or $\mathrm{Y}$ cross-sectional shape to a paired, smaller element in lissamphibians (Figure 1.3). Prior to becoming U- or Y-shaped in cross-sectional view, the sphenethmoid of early-diverging temnospondyls (e.g., Edops, Eryops) is a large, tube-shaped element (Romer and Witter, 1942; Schoch and Milner, 2014). In Eryops the sphenethmoid is fused with the basisphenoid and, together with the lateral sphenoids, is robust, surrounding the entire midbrain and anterior brain regions and enclosing cranial nerve II (optic nerve) and possibly other nerves (e.g., cranial nerves III and IV; Dempster, 1935). The dissorophoid sphenethmoid is less robust, less well-ossified posteriorly, and open dorsally yielding a U(e.g., Doleserpeton [Sigurdson and Bolt, 2010], Acheloma [Polley and Reisz, 2011]) or Y- 
cross-sectional shape (e.g., Rubeostratilia [Bourget and Anderson, 2013]) (Figure 2.4, Node A). The sphenethmoid of all members of Lissamphibia is reduced to a pair of small bones that lack a ventral floor adjoining left and right sides. Even in caecilians and Eocaecilia, where what is termed the sphenethmoid has a median component in the anterior region (e.g., the unpaired mesethmoid and basiethmoid), the posterior portion that is homologous with the sphenethmoid of other taxa (i.e., the orbitosphenoid portion) is a paired element that lacks an ossified ventral floor (Wake and Hanken, 1982; Müller, 2006; Maddin, 2011; Maddin et al., 2012b). In frogs and salamanders, the element is so reduced that only the small elements in the interorbital region remain (Maddin, 2011). The optic nerve is rarely enclosed within the reduced lissamphibian sphenethmoid. The analysis performed here reveals the absence of an ossified ventral floor is a loss that occurs at the base of Lissamphibia (Figure 2.4, Node D). It further appears as though the cartilaginous precursor of the ventral portion of the sphenethmoid is also lost in lissamphibians (de Beer, 1937; Rose, 2003). This latter feature would be difficult to evaluate in fossil taxa, and so the distinction between sphenethmoid ventral cartilage present but not ossified, and cartilage totally absent could not be made here in the character definitions.

In several cases, on top of the losses reconstructed in my analyses, additional reductions in the size and extent of the basioccipital and basisphenoid ossifications also occur. These details would be impossible to determine with certainty in the fossil record, and so were not included in the official characters as discrete states, but are instead described here. The first is the reduction of the basioccipital from an ossified element to an absent element at the base of the amphibamiform clade (Figure 2.4, Node B). Temnospondyls have generally been noted to have a reduced basioccipital that fails to 
contribute to the occipital condyle in many lineages; however, the degree of ossification is variable. For example, Edops, a basal temnospondyl, has a large, heavily ossified basioccipital that participates in the occipital condyle (Romer and Witter, 1942; Schoch and Milner, 2014). Schoch (1999) noted that the basioccipital is reduced from a large element in taxa such as Eryops, to a smaller element in dissorophoids (Figure 2.4, Node A), where the basioccipital makes minimal contributions to the occipital condyles and is reduced in the posterior braincase region. Both basal Amphibamiformes taxa Micropholis (Schoch and Rubidge, 2005) and Pasawioops (Fröbisch and Reisz, 2008) have been described as possessing a cartilaginous basioccipital, and Doleserpeton (Sigurden and Bolt, 2010) is described as having a basioccipital that is impossible to distinguish from the exoccipitals. In Doleserpeton this is perhaps indicative of the complete absence of the basioccipital bone and cartilage. Schoch (2018) coded Tersomius, Micropholis, Eoscopus, Amphibamus, and Doleserpeton as lacking a basioccipital, noting this includes loss of the basioccipital cartilage. Additionally, adult branchiosaurids (Schoch, 2018) lack a basioccipital bone. However, the character state may be more variable for extant lissamphibians because although many sources note the absence of an ossified basioccipital, and its cartilaginous precursor (Duellman and Trueb, 1994; Schoch and Milner, 2004; Schoch, 2018), some older descriptions indicate a cartilage referred to as a basioccipital in some frogs and less often in salamanders ([Parker, 1876] and sources synthesized within [Rose, 2003]). Most recent descriptions do not mention a basioccipital as contributing to the chrondrocranium nor the ossified braincase in lissamphibians (e.g., Bonebrake and Brandon, 1971; Carroll and Holmes, 1980; Trueb and Hanken, 1992; Lukas and Olsson, 2018). Clearly this region of the skull requires further investigation to 
determine the homology of the structure termed basioccipital by some authors and determine with certainty if this element is completely lost (including cartilage precursor) in extant lissamphibians. In the meantime, based on more recent assessments of its presence/absence (e.g., Lukas and Olsson, 2018), the ancestral character state reconstructions unanimously placed the complete loss of the basioccipital at the base of Amphibamiformes.

Next is a reduction of the basisphenoid. The basisphenoid typically forms the osseous walls and support to the hypophyseal region of the brain (i.e., sella turcica; de Beer, 1937; Romer, 1962). The basisphenoid also undergoes a reduction from an extensive element in Eryops to a smaller element in the dissorophoid Kamacops (Figure 2.4, Node A) and then to a rudimentary ossified element in the amphibamid Tersomius (Figure 2.4, Node B) (Schoch, 1999). Branchiosaurids have a cartilaginous basisphenoid (Boy, 1972; Boy and Sues, 2000), whereas lissamphibians have been described as lacking the basisphenoid, including its cartilaginous precursor (Duellman and Trueb, 1994; Müller, 2006). Data concerning the basisphenoid are unfortunately not available for other higher Amphibamiformes, Georgenthalia and Gerobatrachus. My analysis suggests this loss took place at the base of Lissamphibia (Figure 2.4, Node D). However, the reduction of the basisphenoid may have been more gradual before its eventual loss. This is difficult to characterize in the amphibamiform lineage, as it is an internal structure often not visible in many taxa but the data on presence/absence and morphology when present may improve as more $\mathrm{CT}$ datasets become available.

Finally, the loss of foramina for the hypoglossal nerve (n. XII) in the exoccipitals also occurs at (e.g., in the likelihood result on the parsimony and Bayesian trees) or close 
to (e.g., in the parsimony result on the parsimony tree) the base of Lissamphibia (Figure 2.4, Node D). The ambiguous location obtained in the parsimony-based reconstruction on the parsimony tree is likely the result of missing data in the matrix. Neither Georgenthalia nor Gerobatrachus can be scored for this character, and thus create the possibility that they too lack n.XII foramina. It may in fact be the case, however, that these taxa do possess n.XII foramina, which would yield a result consistent with the likelihood analyses performed on both the parsimony and Bayesian trees. I am inclined to advocate for this latter interpretation given the support from two out of three of the analyses and that all dissorophoids (except for lissamphibians), and all tetrapods examined thus far, possess foramina for n.XII. The loss of n.XII foramina is consistent with the trend of braincase reduction, as it suggests a reduction may have occurred in the number of occipital somites that would have enclosed n.XII within the braincase, as in amniotes (Maddin, 2015; Maddin et al., 2020). The presence of n.XII foramina in all non-lissamphibian dissorophoids is interpreted as corresponding to the presence of an amniote-like number of occipital somites contributing to the braincase (e.g., four or more [Augier, 1931; de Beer, 1937; Maddin et al., 2020]). The absence of foramina for n.XII in lissamphibians, where n.XII exits posterior to the braincase due to the lack of incorporation of the somites associated with it (e.g., anteriormost three or fewer [Augier, 1931; de Beer, 1937; Piekarski and Olsson, 2007; 2014]), appears to be a trait unique to lissamphibians in this analysis. However, this remains to be confirmed when Georgenthalia and Gerobatrachus can be confidently scored for this character, at which time the loss would remain a derived event that simply took place a little earlier in the evolution of lissamphibians. 
The reduction of all of these elements in temnospondyls and in dissorophoids has been used as support of a temnospondyl ancestry for lissamphibians, as lepospondyls do not show the same reductions in the braincase during their evolution (Schoch and Milner, 2004; Maddin et al., 2012a; Maddin, 2015). Many lepospondyls do share a number of losses with lissamphibians, however, these tend to be components of the dermal skull (Marjanović and Laurin, 2015), which tend to experience higher rates of homoplasy than the braincase. Of further importance is the result that each of the loss events identified here currently appear to be unique to the lissamphibian lineage and have occurred in the braincase, a region known to have a stronger phylogenetic signal than other regions of the skull or postcranium (e.g., Cardini and Elton, 2008; Goswami and Polly, 2010; Maddin et al., 2012b; Brazeau and de Winter, 2015; Maddin, 2015). I admit that the loss of nonbraincase skull bones is something that happens commonly over the course of tetrapod evolution. For example, in addition to the losses discussed here, all lissamphibians or members within Lissamphibia have lost numerous skull and lower jaw bones additional to those described in the current analysis, including the jugals, supratemporals, intertemporals, postfrontals, postorbitals, postparietals, tabulars, coronoids, splenials, and surangulars, among others (e.g., Boy and Sues, 2000; Benton, 2014; Schoch, 2014a). These widespread losses often resemble losses in other tetrapod lineages but have happened in a convergent context. This context is especially relevant when similar processes, such as certain heterochronic processes or body size reduction, also happen convergently and bring along with them their correlated morphological outcomes. This is particularly important here because 1) the origin of the lissamphibian form has been tied to heterochronic processes that bear predictable outcomes (i.e., paedomorphosis) and 2) controversy 
surrounding their origins has been complicated by a lack of knowledge regarding which of these predictable features are homologous versus homoplastic.

The patterns of reduction and ultimately morphological and compositional simplification noted here as characterizing the origin of the lissamphibian braincase is not limited to this part of the body. There are numerous examples of additional reduction and losses of the dermal elements contributing to the skull as well as the dentition leading to lissamphibians (Boy and Sues, 2000; Benton, 2014; Schoch, 2014a). For example, the loss of dentition on the basal plate of the parasphenoid, and the fangs on the palatal elements (e.g., vomer, palatine, and ectopterygoid) are all lost at various stages within Amphibamiformes leading to lissamphibians. The driving forces behind the unique pattern of braincase reduction in lissamphibians, in spite of the resilience of this region to morphological change, are ultimately unknown and my research represents an important first step in understanding the unique lissamphibian body plan. Importantly, my analysis contributes a piece to the broader story of simplification in the origin of lissamphibian form in general.

\section{5: Conclusions}

The analysis performed herein demonstrates that the reduced condition of the lissamphibian braincase is a derived state compared to its closest temnospondyl relatives. My broadly sampled phylogenetic analysis reveals the stepwise sequence of three loss events and one element reduction as part of the evolutionary transformation leading to the origin of the form of the lissamphibian braincase. Furthermore, the significant steps in the sequence, as well as the sequence of braincase reduction itself appears to be unique to lissamphibians, consistent with an amphibamiform temnospondyl origin. While previous 
research in early tetrapods (e.g., stereospondyls) has indicated that the degree of braincase ossification has little phylogenetic significance (Clack and Holmes, 1988; Schoch, 2002), in more recent years and in many lineages (e.g., lissamphibians, acanthodians, and mammals), the braincase has revealed itself to be an important tool for phylogeneticists, as it is strongly influenced by early developmental constraints and appears to be less influenced by external factors than other skull components (e.g., the craniofacial skeleton) (Cardini and Elton, 2008; Goswami and Polly, 2010; Maddin et al., 2012b; Brazeau and de Winter, 2015; Maddin, 2015). My research shows that the braincase is indeed a useful tool for at least the temnospondyl lineage leading to lissamphibians. The ancestral character state reconstructions reveal for the first time within an empirical context that the lissamphibian braincase and occipital morphology are secondarily derived, as suggested by previous fossil workers (e.g., de Beer, 1937; Romer and Edinger, 1942; Clack and Milner, 2009). Therefore, the ancestral occipital morphology for Tetrapoda is that of a more heavily ossified braincase with more elements, similar to what is observed in extant amniotes. For the overarching theme of this thesis, I have focused on character transformations that may shed light on the developmental condition of the braincase, specifically that of the occiput, and thus the location of the skull-neck boundary. Unfortunately, a clearer picture of the correspondence between occipital bone presence/absence and numbers of occipital somites is lacking at this time. However, elucidating the correspondence between occipital bone presence/absence and numbers of somites that contribute to the occiput in lissamphibians is in part the goal of Chapter 3. 


\section{Chapter 3: Normal skull development in two amphibian model organisms, Ambystoma mexicanum (salamander) and Xenopus laevis (frog)}

\section{1: Introduction}

In Chapter 2, I tested the hypothesis that the lissamphibian braincase morphology, specifically at the occiput, was secondarily derived in its relatively reduced state in comparison with that of amniotes. The phylogenetic analyses and the ancestral state reconstructions I performed supported the hypothesis that the reduced lissamphibian braincase is secondarily derived. However, the overall reduction of braincase bones in the lineage leading to lissamphibians over deep evolutionary time is only part of a complex story of skull and skull-neck boundary evolution, of which many aspects still require clarification. For example, the relationship between occipital bone presence/absence and the number of occipital somites present is currently not well understood in extant lissamphibians.

The elements that comprise the occiput, along with elements that comprise the vertebral column, are derived from paraxial mesodermal structures called somites (Hall, 2005; Olsson et al., 2005; Gomez and Pourquié, 2009; Gilbert, 2010). Somitogenesis, the developmental process where somites form from the paraxial mesoderm along the anteriorposterior axis, is the earliest, physical sign of segmentation in a developing embryo (Dequéant and Pourquié, 2008). During somitogenesis, somites rhythmically form within the presomitic paraxial mesoderm at regular intervals (e.g., every 30 minutes in zebrafish [Schröter et al., 2008], every 120 minutes in mice [Tam, 1981]). At the end of somitogenesis, the embryo exhibits the products of segmentation - i.e., the somites (Figure 
1.1). The number of somites present in a vertebrate along the anterior-posterior axis is highly lineage, and even species, specific, and ranges from small numbers of somites (e.g., fifteen in frogs by the time they reach the tail bud stages of development [Richardson et al., 1998]) to large numbers of somites (e.g., several hundred in snakes [Gomez et al., 2008]).

In addition to segmenting the embryo along the anterior-posterior axis, somites also determine the migration pathways of the neural crest and guide the development of spinal nerves, making them incredibly important developmental structures to a variety of anatomical systems (Keynes and Stern, 1984; Kalcheim, 2011; Kuratani et al., 2018). Following somitogenesis, the somites further differentiate into dermatome, myotome and sclerotome (Aoyama and Asamoto, 1988; Gomez and Pourquié, 2009; Fleming et al., 2015). The dermatome and myotome go on to differentiate into dermis and skeletal muscles, respectively, whereas the sclerotome differentiates into cartilage and then bone (Dockter, 2000; Gomez and Pourquié, 2009; Fleming et al., 2015). Following segmentation into dermatome, myotome, and sclerotome, somites then undergo another round of segmentation in the process known as resegmentation (Remak, 1855). During resegmentation, the sclerotomal portions of the somites are segmented into anterior and posterior halves, which then subsequently recombine to form skeletal structures that are offset from the myotome-derived muscles by half a segment. Motor axons that go on to form nerves are associated with the anterior half of somites (Keynes and Stern, 1984; Dockter, 2000).

The anterior, or occipital somites, are associated with various soft tissues within the head and the skeletal tissues of the occipital arch. For example, the myotomal portions of 
the occipital somites go on to form the hypobranchial muscles (i.e., the muscles of the tongue). In chicken, the myotomes of occipital somites two through five (plus a portion of the first trunk myotome, somite six) contribute to these muscles (i.e., the glossal and infrahyoid muscles [Hazelton, 1970; Couly et al., 1993; Huang et al., 1999]). In Ambystoma mexicanum, only the myotomes of the three occipital somites contribute to the hypobranchial muscles (i.e., m. geniohyoideus and m. rectus cervicis [Piekarski and Olsson, 2007]). In terms of the occipital arch, the sclerotomal portions of the occipital somites contribute to the structures that make up the occipital arch and have been the subject of study in the context of skull evolution for a long time. However, even at present there has been a failure to come to a consensus on how many somites contribute to the occipital arch, and thus the skull, even when studying the same species. For example, three (Hunter, 1935) or four (Chiarugi, 1890) somites may contribute to the occipital arch in rabbits. This discrepancy in the literature is likely the result of several different factors, including differing definitions of what consists of a somite (e.g., Butcher, 1929; Kuratani et al., 1999), short developmental time periods studied that fail to account for the transient nature of somites where anterior somites have differentiated and disappeared while posterior somites are still forming (e.g., Elliot, 1907; Dawes, 1930), or because at various stages during development anterior somites may still be present but covered by other structures, such as the migrating neural crest (e.g., Piekarski, 2009). More recent long term, fate mapping techniques have come to a consensus for chicken, a commonly studied model organism for amniotes, confirming that the first five somites contribute to the occiput in chicken (e.g., Couly et al., 1993; Huang et al., 2000). In A. mexicanum, three somites have been confirmed to contribute to the occiput (e.g., Piekarski and Olsson, 2007; 2014). 
Similar fate mapping studies have not been conducted in frogs, and the present study aims to fill this gap. For both A. mexicanum and Xenopus laevis, two widely used lissamphibian model organisms, it is necessary to conduct long term fate mapping of proposed occipital somites in order to allow more meaningful comparisons of skull-neck boundary evolution (and its consequences) to be made across Tetrapoda.

Further complicating our understanding of occipital development and thus evolution is the scarcity of complete developmental descriptions of the skull in the literature for the relatively understudied amphibians. Both A. mexicanum and X. laevis are widely used model organisms with long and diverse histories in genetics, development, and, especially for A. mexicanum, regenerative biology (e.g., Maden et al., 1983; Gardiner et al., 1995; Yang et al., 1999; Kragl et al., 2009; Harland and Grainger, 2011; Godwin et al., 2013; Piekarski et al., 2014). However, in recent years, both organisms have become important models in more diverse studies that focus more broadly on skull development (e.g., Sefton et al., 2015; Charbonneau et al., 2016; Maddin et al., 2016; Lukas and Olsson, 2018; Sader et al., 2018); yet, both organisms lack a formal, staging table that documents the timing and variation of events in the development of the skull.

Early development in A. mexicanum (i.e., from fertilization to hatching and prior to forelimb bud formation) has been described extensively (e.g., Armstrong and Malacinski, 1989; Bordzilovskaya et al., 1989; Schreckenberg and Jacobson, 1975); however, the only staging table that describes post-hatching development (i.e., the stages that record the vast majority of events in skull development) pertains to stages defined by events in limb development (Nye et al., 2003). While the Nye et al. (2003) staging table is a valuable resource for A. mexicanum researchers, the role of $A$. mexicanum in research is ever 
expanding beyond its historic use in limb regeneration studies (e.g., Charbonneau et al., 2016; Maddin et al., 2016; Sader et al., 2018; Sefton et al., 2015), and as such, researchers studying the cranial anatomy of $A$. mexicanum are currently unable to quickly and accurately assign stages to material consisting of skulls only or are lacking the means to assign a stage to specimens that are either artifactually or pathologically missing limbs. Description of skull development in A. mexicanum complementing current stages based on limbs would solve these and other limitations presented by current staging tables.

For X. laevis, the current most widely used staging table is that of Nieuwkoop and Faber (1994), which focuses almost exclusively on external features. Subsequent researchers have attempted to augment the Nieuwkoop and Faber (1994) staging table using the developing chondrocranium, dermatocranium, or muscles (e.g., Trueb and Hanken, 1992; Ziermann and Olsson, 2007; Zahn et al., 2017; Lukas and Olsson, 2018). While these efforts also provide valuable information, they either do not provide photographs or illustrations of every stage for the duration of development, which is an important feature of staging tables, or they present developmental data within a discrete staging system, which cannot be easily integrated into the widely used staging table of Nieuwkoop and Faber (1994). To be able to compare normal development to perturbed development in future studies (i.e., those in Chapter 4), it is necessary to re-examine chondrification and ossification patterns of normal skull development in X. laevis for each stage of Nieuwkoop and Faber (1994) and in A. mexicanum for each stage of Nye et al. (2003), while also noting instances of developmental variation, which is additionally poorly documented in these species. 
Further to the problems noted above, the cranial nerves associated with the skullneck boundary, specifically the hypoglossal nerve complex, have not been well described in A. mexicanum nor in X. laevis in how they relate to cartilaginous or ossified structures along the anterior-posterior axis. The hypoglossal nerve innervates the tongue and associated throat musculature as well as the muscles of the hyobranchial apparatus (Strong, 1895; Barnard, 1940; Roth et al., 1984). While the hypoglossal nerve is considered the last, or most posteriorly located, cranial nerve in amniotes, in amphibians the nerve that innervates the tongue and associated structures is typically referred to as the first spinal nerve (and sometimes as the first two or three spinal nerves), despite its morphological resemblance to the hypoglossal nerve in amniotes and that it innervates the same structures (Barnard, 1940; Maddin et al., 2020). In amniotes, nerves associated with the occipital somites have ventral motor roots but lack dorsal sensory roots. This morphology is also true of the first, and sometimes second, spinal nerves in amphibians even though they are not associated with the occipital somites (Fürbringer, 1897; Barnard, 1940; Edwards, 1976). A recent review by Maddin et al. (2020) found that while the number of somites contributing to the occiput varies, due to reasons already outlined above, when one aligns the somites associated with the hypoglossal nerve complex, the variation in location of the skull-neck boundary is actually reduced. Under this scheme, three conditions are revealed: an amniote condition, a condition present in salamanders and caecilians, and a condition present in frogs (Maddin et al., 2020). To further understand the information the hypoglossal nerve can provide to the story of skull-neck boundary evolution, a closer look at this nerve's association with the skull is required. 
The morphology of the hypoglossal nerve as it relates to the chondrocranium, and ossified elements is well documented in amniotes. In amniotes, the roots of the hypoglossal nerve exits the skull via a variable number of foramina present on the lateral or ventral surface of the exoccipital (Figure 1.4; Kuratani et al., 1988; Higashiyama et al., 2016; Hüppi et al., 2019; Sakamoto, 2019). In amphibians, it has been suggested that at least the first spinal nerve, and possibly contributions from the second and/or second and third spinal nerves, is homologous to the hypoglossal nerve of amniotes (e.g., Platt, 1897; Elliot, 1907; Norris, 1908; Norris, 1913; Barnard, 1940; Herrick, 1948; Wake and Lawson, 1973; Stuesse et al., 1983; Roth et al., 1984; Wake, 1992; Naumann and Olsson, 2018). In salamanders, the first, and possibly the second, spinal nerve contributes to the hypoglossal nerve in plethodontids and Necturus and it extends laterally from the spinal cord just anterior to the first vertebral element (Figure 1.4; Platt, 1897; Wake and Lawson, 1973; Roth et al., 1984). In Ambystoma tigrinum, Amphiuma, and Siren lacertina the first spinal nerve that contributes to the hypoglossal is associated with the second, third, and fourth myotomes and the roots exits the spinal cord together via a foramen on the first vertebral element (Figure 1.4; Norris, 1908; Norris, 1913; Herrick, 1948; Edwards, 1976). In frogs, the first, second, and possibly third spinal nerves contribute to the hypoglossal nerve complex in X. laevis (Naumann and Olsson, 2018), whereas in Rana the second spinal nerve contributes to the hypoglossal where it extends laterally from the spinal cord in the space between the first and second vertebral elements (Figure 1.4; Elliot, 1907; Barnard, 1940; Stuesse et al., 1983). In caecilians, the first through third spinal nerves variably contribute to the hypoglossal and its roots exit the spinal cord together via a foramen in the first vertebral element (Epicrionops, Uraeotyphlus) or pass between the second and third 
vertebral elements (Ichthyophis, Scolecomorphus), indicating variability in caecilians (Wake, 1992). This variability noted in caecilians, which is greater than that found in other amphibians and in amniotes, is likely the result of their limbless body plans and simple tongue morphologies when compared to other amphibians (Wake, 1992). However, as with the position of the skull-neck boundary, lineage specific patterns emerge for the position of where the hypoglossal nerve exits the spinal cord in amphibians: the salamander morphology where the hypoglossal nerve passes laterally either via a foramen in the atlas or anterior to the atlas, the frog morphology where it passes laterally between the first and second vertebral elements, and the caecilian morphology, which is variable. The frog and salamander hypoglossal nerve morphology, along with the well documented amniote morphology, are summarized in Figure 1.4. Unfortunately, without more descriptive studies of skull anatomy and the associated cranial nerves in lissamphibians, these proposed patterns are not certain. In the present study, I aim to describe the skull anatomy as it relates to the hypoglossal nerve complex in two lissamphibian model organisms, $A$. mexicanum and $X$. laevis.

Here, there are three goals to accomplish using the amphibian model organisms $A$. mexicanum and X. laevis: 1) describe the early development of the skull, specifically the occipital region, in terms of somitic contributions, 2) describe the later stages of skull development noting the timing of appearance of key occipital structures, augmenting already published staging tables based on external or limb morphology, and 3) describe the hypoglossal nerve morphology in the occipital region as it relates to the skull and vertebral elements at the skull-neck boundary. These baseline, normal developmental data, including intraspecific variation in the timing of when elements appear and their 
morphological variation within each stage, will be invaluable for future studies that focus on perturbed development, such as my study outlined in Chapter 4 . Without a baseline developmental series and baseline morphology for structures such as the hypoglossal nerve complex for these lissamphibian model organisms, it would be impossible for future studies to determine if their results fall within normal developmental variation or the extent to which development has been perturbed.

\section{2: Methods}

\subsection{1: Animal care and breeding}

\section{Ambystoma mexicanum}

Adult A. mexicanum breeding pairs are maintained as part of the breeding colony in the Maddin Lab at Carleton University, Ottawa, Canada (original source: Ambystoma Stock Center, Lexington, KY). All A. mexicanum are housed and cared for in accordance with the Canadian Council on Animal Care approved animal use protocol (AUP \#102951). Clutches of A. mexicanum embryos were obtained from natural breeding events between male and female leucistic mature adults. Embryos were dejellied (i.e., the protective membranes surrounding the embryo were removed) manually using forceps after neural tube closure and kept in agar-lined Petri dishes filled with autoclaved $40 \%$ Holtfreter's solution in an incubator at $18^{\circ} \mathrm{C}$. At approximately stage 45 (Bordzilovskaya et al., 1989) larvae were transferred to containers containing $20 \%$ Holtfreter's solution and maintained at $18^{\circ} \mathrm{C}$ on a 12 -hour day and 12 -hour night light cycle. Once able to feed, hatched larvae were fed a diet of larval brine shrimp once daily. Embryos were staged according to Bordzilovskaya et al. (1989) and larvae were staged according to Nye et al. (2003). 
Xenopus laevis

Adult X. laevis breeding pairs are maintained as part of the breeding colony in the Maddin Lab at Carleton University, Ottawa Canada (original source: Marine Biology Laboratories, Woods Hole, MA). All X. laevis are housed and cared for in accordance with the Canadian Council on Animal Care and Carleton University's Animal Care Committee approved animal use protocol (AUP \#102952). In order to induce spawning, female $X$. laevis were initially injected with 50 IU (international units) of human chorionic gonadotropin (HCG; Sigma C1063). After 48 hours, the female was injected again with $500 \mathrm{IU}$ of HCG. At the time of the female's second injection, a male was injected with 50 IU of HCG. Both frogs were then placed together in a nuptial tank with artificial plants and tubing. The next morning, embryos were collected and chemically dejellied using a $2 \%$ solution of buffered L-Cysteine free base (Fisher Scientific BP376100). X. laevis embryos were stored in agar-lined Petri dishes filled with autoclaved 10\% Holtfreter's solution. At approximately stage 46 (Nieuwkoop and Faber, 1994), tadpoles were transferred to small tanks filled with $20 \%$ Holtfreter's solution. Once able to feed, tadpoles were fed a diet of Tropical Pro Defence Hi-Protein food for fry once daily. Embryos and tadpoles were staged according to Nieuwkoop and Faber (1994).

\subsection{2: Somite cell-lineage tracing}

Previous research in A. mexicanum showed that somite one is too small and irregularly shaped for accurate injection (Piekarski, 2009). In X. laevis, the first somite is also much smaller and irregularly shaped than subsequent somites (Youn and Malacinski, 1981). Somite one may also eventually merge with somite two, making it difficult to confidently track derivatives of a potential somite one (Piekarski, 2009). Fortunately, 
somites three and two are the somites reported to contain the skull-neck boundary in salamanders and frogs, respectively (de Beer, 1937; Piekarski, 2009), and both are clearly identifiable somites in both species. As such, somites two and three for A. mexicanum and somite two for $X$. laevis were the focus of the cell-lineage tracing experiments performed here.

To trace the fate of somites, $A$. mexicanum embryos were selected between stages 20 and 25 (Bordzilovskaya et al., 1989; see Appendix D for specimen numbers) and $X$. laevis embryos were selected between stages 17 and 24 (Nieuwkoop and Faber, 1994; see Appendix D for specimen numbers), which correspond to stages with clearly visible somites. Interestingly, previous cell-lineage tracing work found that the pronephros is a reliable landmark for identifying and counting somites in A. mexicanum (Piekarski, 2009). Using the pronephros as a landmark eliminated the risk of miscounting somites depending on whether or not a transient first somite was present and because at some developmental stages somite one and part of somite two are covered by the second branchial neural crest stream (Piekarski, 2009). Without a landmark, the first, completely visible somite at some stages is actually somite three. In A. mexicanum, the anterior limit of the pronephros is located at the border between somite three and four (Figure 3.1A, B; Piekarski, 2009). The pronephros was also found to be a consistent landmark in $X$. laevis, with its anterior limit being located at the border between somite two and three (Figure 3.1C, D). 


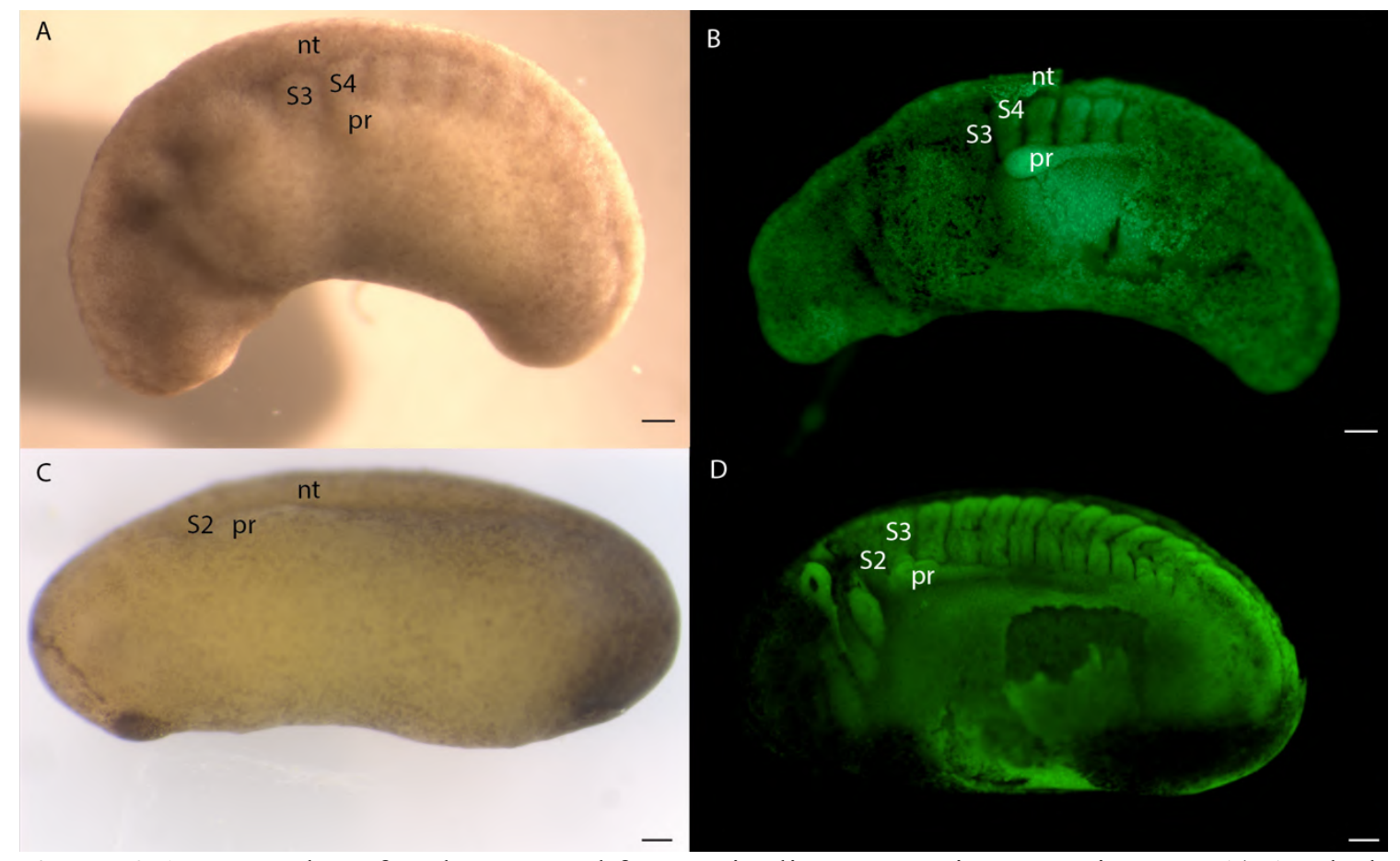

Figure 3.1. Examples of embryos used for somite lineage tracing experiments. A) A whole stage $25 \mathrm{~A}$. mexicanum embryo. B) A stage $28 \mathrm{~A}$. mexicanum embryo with the ectoderm removed. C) A whole stage $26 X$. laevis embryo. D) A stage $23 X$. laevis embryo with the ectoderm removed. The anterior limit of the pronephros (pr) is located at the border between somite three (S3) and somite four (S4) in A. mexicanum and between somite two (S2) and somite three (S3) in X. laevis. The neural tube (nt) is the dorsal most structure. Scale bars equal $100 \mu \mathrm{M}$.

Both $A$. mexicanum and $X$. laevis embryos were injected with Fluorescein-labeled dextran (25 mg/ml, 10,000 molecular weight, Thermo Fisher Scientific D34679) using a micromanipulator and an Eppendorf FemtoJet $4 \mathrm{x}$ autoinjector connected to pressurized air set to 150 seconds and 300 psi. Embryos were injected in the left side in the central part of one somite in order to avoid labelling other tissues. Immediately after injection, and at 24hours and 48-hours post-injection, accuracy was checked using a Zeiss SteREO Discovery.V8 stereo microscope with a DSRed filter. In photographs, fluorescence was artificially coloured red in the Zeiss Zen software. Only those embryos that had strong fluorescence in one somite were considered for further analysis. After 48-hours, embryos were checked and photographed once a week. Embryos were allowed to develop under 
normal conditions post-injection, until the A. mexicanum embryos reached stages 47 to 56 (Nye et al., 2003) and the $X$. laevis embryos reached stage 58 (Nieuwkoop and Faber, 1994). These corresponded to stages when skeletal structures, specifically occipital and vertebral elements, were present. See Appendix D for specimen numbers of surviving embryos for this dataset.

An unpublished dataset (courtesy of Maddin and Piekarski) of A. mexicanum specimens had GFP (green fluorescent protein)-positive somites grafted for long term fate mapping. Two clutches were obtained for this dataset that were the result of a cross between a leucistic individual and a ubiquitous GFP-positive transgenic individual of $A$. mexicanum. Surgeries were performed on embryos between stages 21 and 27 (Bordzilovskaya et al., 1989). GFP-negative and GFP-positive embryos were first stage matched. The epidermis on the left side of both embryos was cut ventrally and peeled up, dorsally. The somite of interest was first removed from the host (GFP-negative) embryo. Next, the corresponding somite from the donor (GFP-positive) embryo was removed and it was placed in the host embryo. The epidermis was pulled back into place over the somites and held in place by a small piece of cover slip glass. Within an hour, host embryos had healed from the transplant surgery and were photographed for accuracy. Twenty-four- and 48-hours post-surgery, accuracy was followed up by checking and photographing using a fluorescence stereo microscope. Embryos were then allowed to develop under normal conditions.

Once specimens reached the desired stage (i.e., when occipital skeletal structures of interest were present based on the bone and cartilage whole-mount stained data), they were anesthetized by adding a few drops of 4\% MS-222 (ethyl 3-aminobenzoate methanesulfonate [Sigma-Aldrich E10521]) to their container. Once vital signs 
disappeared the specimens were then fixed in $4 \%$ paraformaldehyde overnight at $4^{\circ} \mathrm{C}$. Following fixation, specimens were dehydrated through a methanol in phosphate buffered solution (PBS) series (one hour in each: $25 \%$ methanol in PBS, $50 \%$, and $75 \%$ ) and stored in $100 \%$ methanol at $-20^{\circ} \mathrm{C}$. Specimens were later embedded in optimal cutting temperature (OCT) compound (Fisher Healthcare 23730571) by rehydrating the specimens through a methanol in PBS series (one hour in each: 75\% methanol in PBS, 50\% methanol, 25\% methanol, $100 \%$ PBS). Specimens were then moved to a $15 \%$ sucrose in PBS solution and left overnight at $4^{\circ} \mathrm{C}$, then placed in a $30 \%$ sucrose in PBS solution and left overnight at $4^{\circ} \mathrm{C}$. Specimens were then left overnight for a third time in a $1: 1$ solution of $30 \%$ sucrose in PBS and OCT compound. The next day, specimens were mounted in an embedding mold filled with OCT compound and quickly frozen using a dry ice in 100\% ethanol bath and then stored at $-80^{\circ} \mathrm{C}$ to preserve fluorescence.

Embedded specimens were sectioned using a HM525 NX cryostat (Thermo Scientific) set to $-20^{\circ} \mathrm{C}$. Slices were 10 microns thick and resulting slides were stored at $80^{\circ} \mathrm{C}$ to preserve fluorescence. Sections were checked for fluorescence using a Zeiss Axio Imager.M2 microscope using the DSRed filter and photographed using a monochrome camera attachment. Fluorescence was artificially coloured green (somite grafted experiments) or red (somite injection experiments) in the Zeiss Zen software.

\subsection{3: Bone and cartilage staining}

In order to visualize normal cartilage and bone development, a developmental series of A. mexicanum specimens (see Appendix D for specimen numbers for the staging series) and $X$. laevis specimens (see Appendix E for specimen numbers for the staging series) that were previously fixed in $10 \% \mathrm{NBF}$ and stored in $70 \%$ ethanol were placed in 
an Alcian blue (8GX, Acros Organics A3157) solution (20 mL acetic acid, $75 \mathrm{~mL}$ of 95\% ethanol, $0.1 \mathrm{~g}$ alcian blue) for one to six hours, depending on the size of the specimen. Following this, specimens were moved through a hydration series (approximately one hour in each: $70 \%, 50 \%, 30 \%$ ethanol diluted in distilled water) before they were transferred into a trypsin solution ( $30 \%$ saturated borate, $1 \%$ trypsin) and incubated at $37^{\circ} \mathrm{C}$ until their tissues were soft and approximately clear. Specimens were then transferred through a series of $1 \%$ potassium hydroxide $(\mathrm{KOH})$ plus glycerol solutions (approximately one hour to one day in each: $3: 1,1: 1$, and 1:3 $\mathrm{KOH}$ to glycerol) for further clearing and were finally stored in $100 \%$ glycerol.

To visualize bone development, a developmental series of specimens were placed into a $1 \%$ Alizarin red S (Sigma-Aldrich A5533) in a $1 \% \mathrm{KOH}$ for one hour. The bone staining step is not size dependent. After bone staining, specimens were moved into a $30 \%$ saturated borate solution with $1 \%$ trypsin, and incubated at $37^{\circ} \mathrm{C}$, until soft tissues were almost completely clear. For storage and to finish clearing, the specimens were transferred through a series of $1 \% \mathrm{KOH}$ plus glycerol solutions (approximately one hour to one day in each: 3:1, 1:1, and 1:3 KOH to glycerol) and stored in $100 \%$ glycerol.

\subsection{4: Nerve staining}

To visualize nerves as they relate to skeletal structures, the regressive nerve staining protocol outlined by Northcutt et al., (2000) was followed for both A. mexicanum and X. laevis specimens. Specimens were first bleached in a 10\% hydrogen peroxide solution for one to two days, then washed in distilled water for two to three hours. Specimens were then macerated in a $3 \%$ trypsin (A. mexicanum) or $1 \%$ trypsin $(X$. laevis $)$ in a $30 \%$ saturated borate solution until the tissues were clear and nerves were visible. To stain the nerves, 
specimens were then put in a $0.5 \% \mathrm{KOH}$ solution for ten minutes, $70 \%$ ethanol solution for ten minutes, then in a saturated and filtered 0.5\% Sudan Black B (Sigma-Aldrich 199664) dissolved in 70\% ethanol solution for 30 minutes. To clear the excess Sudan Black B, specimens were washed in several $70 \%$ ethanol solutions until the nerves and tissues could be differentiated. Then, specimens were placed in a $0.5 \% \mathrm{KOH}$ solution overnight. For long term storage, specimens were transferred through a series of $1 \% \mathrm{KOH}$ plus glycerol solutions (approximately one hour in each: $3: 1,1: 1$, and 1:3 $\mathrm{KOH}$ to glycerol) and stored in $100 \%$ glycerol.

\section{3: Results}

\subsection{1: Somitic contributions to the occipital arch}

\section{Ambystoma mexicanum}

Somite transplant experiments in A. mexicanum confirmed that, in terms of soft tissue contributions, somite two contributes to the m. geniohyoideus and a portion of the m. rectus cervicis (Figure 3.2A, green-labelled structure), while somite three contributes to the $\mathrm{m}$. rectus cervicis and additionally to the first hypaxial muscle (Figure 3.2B, greenlabelled structures). Transplanting somite four confirmed that this somite contributes to the hypaxial and epaxial trunk musculature (Figure 3.2C, green-labelled structures), and not to musculature within the head.

To determine the somitic contribution to the chondrocranium, results are available from both somite injection and somite grafting experiments. Injecting somite two in $A$. mexicanum resulted in the labeling of the anterior portion of the occipital arch, whereas labeling was absent in the posterior portion of the occipital arch and in the axial skeleton (Figure 3.2D, red-labelled cells). 
GFP-positive somite transplant experiments that were also sectioned revealed that somite three contributes to the posteriormost portion of the occipital arch and the anterior portion of the atlas, the first vertebral element in A. mexicanum (Figure 3.2E, green-labelled cells). This corresponds to the articulation between the occipital arch and the atlas, the articulatio occipitalis (Figure 3.2E, green-labelled cells). Strong labelling was also present dorsally in the first two myotomes (Figure 3.2E, green-labelled structures). The sections also confirmed with the whole-mount specimens that the third somite contributes to the ventrally located $\mathrm{m}$. rectus cervicis and the first hypaxial muscle (Figure 3.2E, greenlabelled structures). 


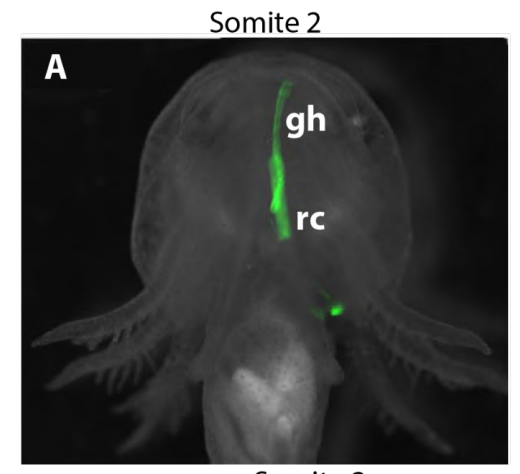

Somite 2

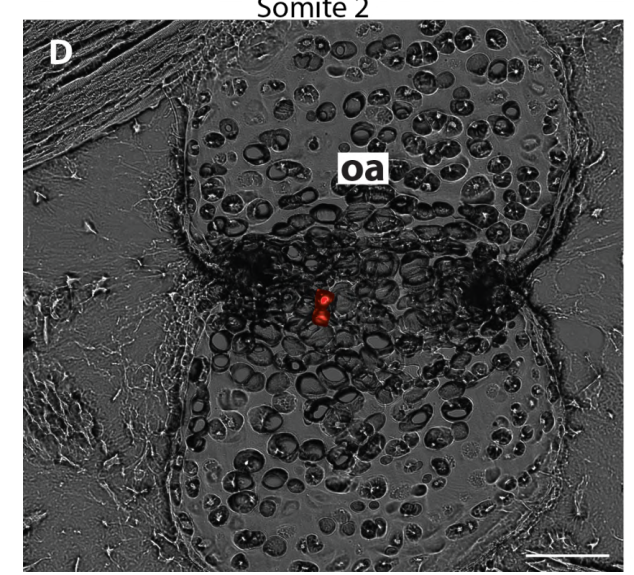

Somite 3
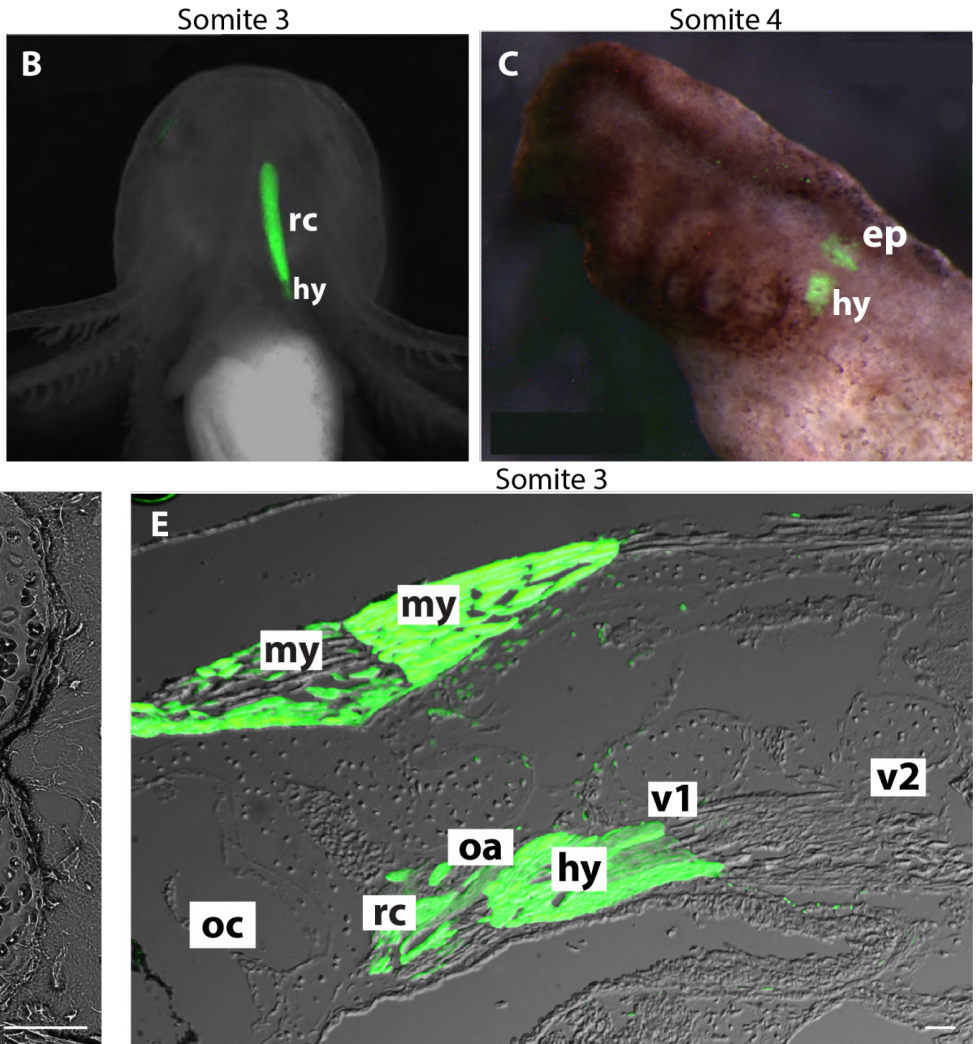

Figure 3.2. A summary of somites that contribute to the muscles, chondrocranium and anteriormost vertebral structures in $A$. mexicanum specimens. A) Ventral view of an $A$. mexicanum specimen with a GFP-positive somite two transplant showing its contribution to the $\mathrm{m}$. geniohyoideus and a portion of the m. rectus cervicis. B) Ventral view of an $A$. mexicanum specimen with somite three transplanted showing its contribution to the $\mathrm{m}$. rectus cervicis and the first hypaxial trunk muscle. C) Lateral view of an A. mexicanum specimen with somite four transplanted showing its contribution to the first hypaxial trunk muscle and the epaxial muscle. D) The occipital arch in an A. mexicanum specimen that had somite two injected with Fluorescein-labeled dextran showing positive cell labelling in the anterior portion of the occipital arch. E) The occipital arch and first two vertebral elements in an $A$. mexicanum specimen with a GFP-positive somite three transplant showing strong label in two myotomes, the rectus cervicis and the first hypaxial trunk muscle. Weaker label is present in the posterior half of the occipital arch and the anterior half of the first vertebral element (the atlas). Scale bars equal $50 \mu \mathrm{m}$. Abbreviations: ep, epaxial trunk musculature; gh, m. geniohyoideus; hy, hypaxial trunk musculature; oa, occipital arch; oc, otic capsule; my, myomere; rc, m. rectus cervicis; v1, first vertebral element; v2, second vertebral element. 


\section{Xenopus laevis}

Whole-mount images taken to confirm presence of labelling in $X$. laevis post injection reveals that injecting somite two results in labelling that appears to span the headtrunk interface (Figure 3.3A, red label). Unfortunately, all the specimens that had somite two injected lost their label before they could be sectioned. A specimen where both somites two and three were labelled together survived and was sectioned. In the whole-mount view, labelling can be seen in the same region as in the whole-mount image of the somite two injected specimen (Figure 3.3A, B, red label). Additional labelling was observed in the jugal foramen after sectioning (likely in the vagus nerve; Figure 3.3D, E, red label). Unfortunately, there was no label present in muscles nor in the skeletal tissues (Figure 3.3D, red label). 
Somite 2

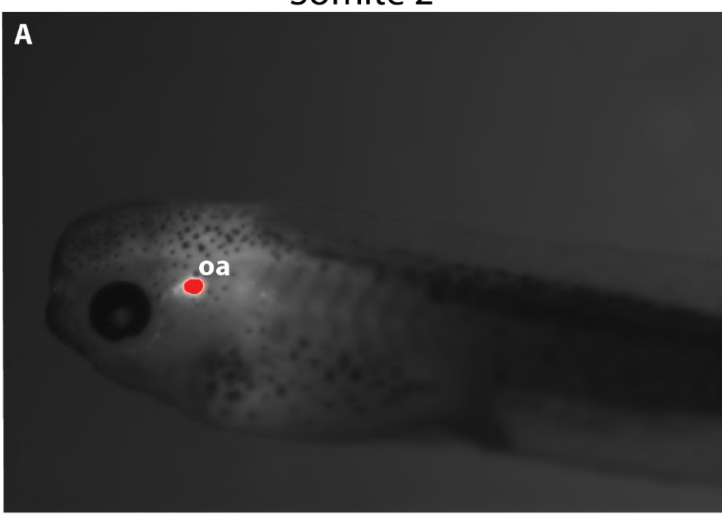

Somite $2 \& 3$ - Occipital region

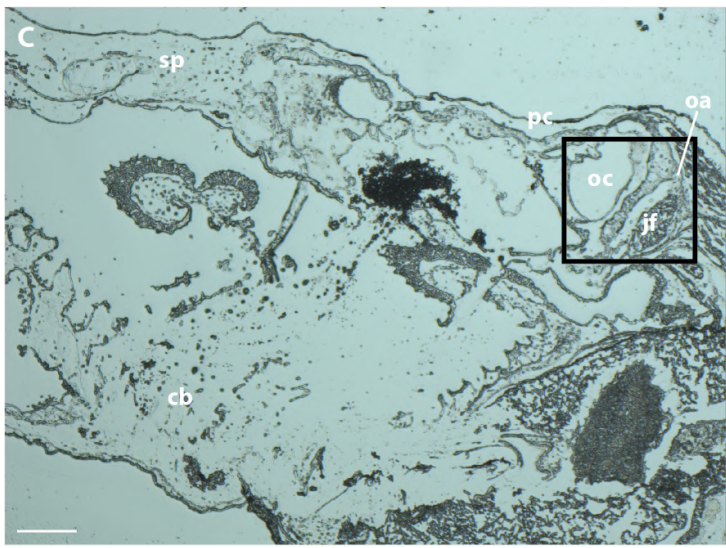

Somite $2 \& 3$ - Occipital region

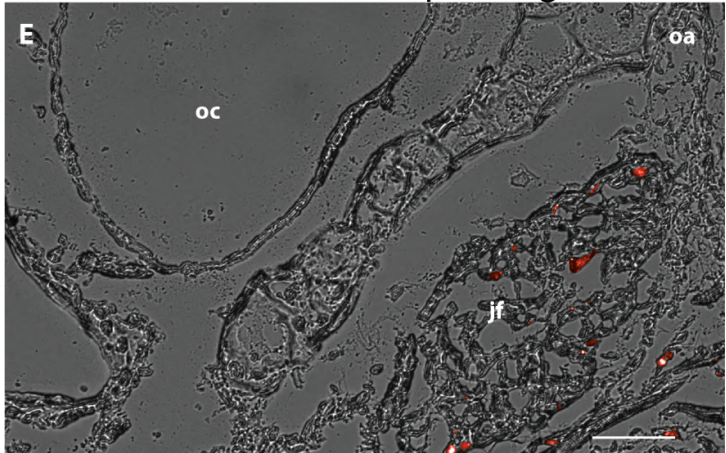

Somite $2 \& 3$

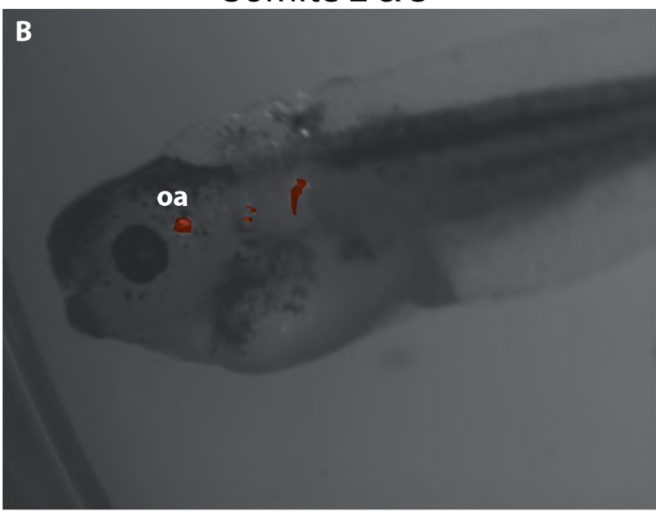

Somite $2 \& 3$ - Post-cranium region

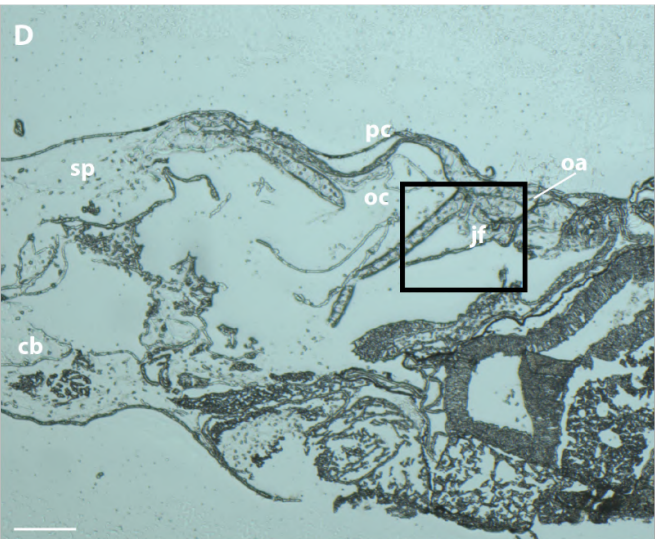

Somite $2 \& 3$ - Post-cranium region

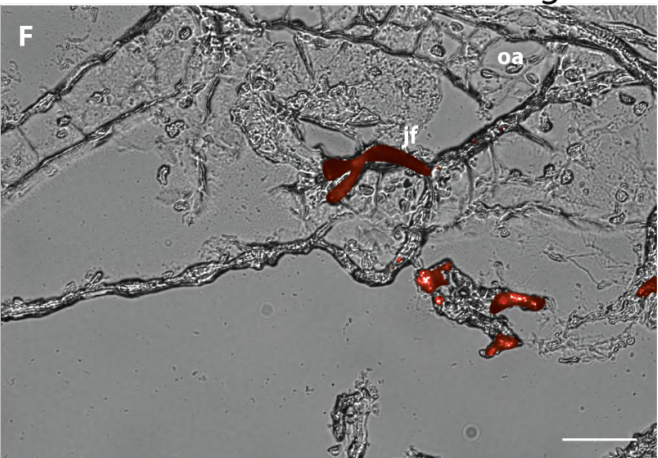

Figure 3.3. A summary of somites hypothesized to contribute to the muscles and occiput in $X$. laevis. A) Lateral view of a X. laevis specimen with somite two injected showing label spanning the approximate area of the skull-neck boundary. B) Lateral view of a $X$. laevis specimen with somites two and three injected showing label both spanning the skull-neck boundary and in the axial skeleton. C) A section of the occipital region from specimen in $\mathrm{B}$ to orientate where the label is occurring in E. D) A section that spans the skull-neck boundary in B to orientate where the label is occurring in F. E) Labelled occipital arch and postcranium elements in an $X$. laevis specimen that had somites two and three injected. F) Labelled postcranium elements in an $X$. laevis specimen that had somites two and three injected. Scale bars equal $200 \mu \mathrm{m}$ in $\mathrm{C}$ and D and $50 \mu \mathrm{m}$ in $\mathrm{E}$ and F. Abbreviations: cb, ceratobranchials; jf, jugular foramen; oa, occipital arch; oc, otic capsule; pc, parachordal cartilage; sp, suprarostral plate. 


\subsection{2: Ambystoma mexicanum normal development of the skull: A complementary staging table}

For consistency, the stages below are descriptions of the skull at developmental stages already described externally by Nye et al. (2003). I use the same stage numbers in order to easily integrate events in the development of the skull with this widely used staging table. Descriptions of specimens begin at stage 44, which is the first stage described in Nye et al. (2003) and one stage before hatching (Bordzilovskaya et al., 1989). By stage 44, all early forming, cartilaginous elements of the skull are present, and the element of most interest for this study, the occipital arch, is consistently present for the first time. The morphology of the cartilaginous elements of the skull does not differ between most stages of development described here, and thus only some figures are illustrated here (i.e., stages $45,47,49,56)$. Ossifications, on the other hand, are described and figured for every stage of Nye et al. (2003). Studied specimens are numbered and listed in Appendix E.

\section{Stages 44 and 45}

At stages 44 and 45, a well-developed chondrocranium is already present in the specimens observed (Figure 3.4A, B). The hyobranchial apparatus, which is important for feeding and respiration, is also present by these stages.

The hyobranchial apparatus is present along the midline of the skull (Figure 3.4A, B). The first basibranchial element is the anteriormost midline element, positioned at approximately the same level, but medial to the eyes, while the second is positioned immediately posterior to the first (Figure 3.4A, B). The basibranchial elements have several other cartilaginous elements that they articulate with, all of which extend laterally and posteriorly away from the basibranchial elements. First, in the anteriormost position on 
either side of the first basibranchial element is the hypohyale (medial, articulates with the first basibranchial) and ceratohyal (lateral, articulates with the hypohyale) elements (Figure 3.4A, B). Posterior to the hypohyale, the hypobranchial element also articulates with the basibranchial elements. The first hypobranchial is associated with the first ceratobranchial element, while the second, third, and fourth ceratobranchial elements are associated with the second hypobranchial (Figure 3.4A, B).

In addition to the hyobranchial apparatus, several other cartilaginous elements are present dorsal to the hypobranchial apparatus. Along the midline of the skull and positioned medially to the eyes but lateral to the basibranchial elements are the paired trabecular cartilages (Figure 3.4A, B). They are large rod-shaped elements that are the most anterior reaching elements in the chondrocranium as they extend past the anterior extent of the eyes. Posteriorly, the trabecular elements have a wider base that reside on either side of the medially located notochord and articulate with the triangular quadrate cartilage laterally (Figure $3.4 \mathrm{~A}, \mathrm{~B}$ ). Immediately posterior to the trabecular cartilages are the paired parachordal cartilages. The parachordal cartilages also reside on either side of the notochord (Figure 3.4A, B). Lateral to the parachordal cartilage are the otic capsules, which also articulate with the posterior portion of the palatoquadrate cartilage (Figure 3.4A, B). The otic capsules are positioned dorsally to the ceratobranchial elements of the hyobranchial apparatus. Posterior to the otic capsule is the occipital arch (Figure 3.4A, B). At stages 44 and 45, these elements are virtually indistinguishable in shape from the paired neural arches in the anterior region of the vertebral column; however, they are slightly smaller than the neural arches. 
In the lower jaw, the two halves of Meckel's cartilage meet at the midline of the skull. Meckel's cartilage curves posterolaterally. Posteriorly, it meets the quadrate cartilage at a level that is slightly posterior to the posteriormost extent of the eyes (Figure 3.4A, B).

No ossifications are evident at stage 44 (Figure 3.5A-C). A couple of tooth crowns are faintly stained in the future coronoid region in at least one specimen at stage 45 (Figure 3.5A-C). In another one of the larger specimens, approaching stage 46 , a faintly ossified coronoid bone was observed on the medial surface on both sides of the lower jaw (specimen not figured).

\section{Stage 46}

The first ossified components of the skull are consistently present by stage 46 . Here, four paired elements are present: vomers, palatines, coronoids, and dentaries (Figure 3.5DF). The tiny vomer occurs in the anterior portion of the future palate, anterior to the eyes. It is a small, circular element with at least one tooth crown present (Figure 3.5D-F). Posterior to the vomer is the first sign of the palatine. It is a small, similarly-sized, circular element (Figure 3.5D-F). In one specimen, only the palatine appears, suggesting the palatines appear before the vomers (specimen not figured). On the dorsomedial portion of Meckel's cartilage, lateral to the level of vomer and palatine, the coronoid is present as a thin ossification (Figure 3.5D-F). In one specimen, the dentary is present as a very small and thin ossification on the anterior margin of Meckel's cartilage. Left and right dentaries are separated from one another medially by a substantial gap (the presumptive symphysis).

In the axial skeleton, the paired neural arches have become more robust than those in stages 44 and 45 and more elements have continued to develop moving posteriorly along 
the anterior-posterior axis. The cartilaginous precursor to the shoulder girdle is consistently present between the first and second paired neural arches (data not shown).

\section{Stage 47}

By stage 47 , another cartilaginous element is consistently present in the anterior region of the head, between the trabecular cartilage elements (Figure 3.4C, D). This element is the internasal plate. At this stage, the internasal plate is a faint, narrow, U-shaped element (Figure 3.4C, D). In the posterior region of the head, the parachordal cartilage has extended posteriorly on either side of the notochord, resulting in a distinctive median notch in the element (Figure 3.4C, D). The occipital arch is more robust and has grown in an anterior direction towards the otic capsules (Figure 3.4C, D).

The ossified vomers and palatines are still roughly the same size as one another, and each bear about four to five teeth (Figure 3.5G-I). In one specimen, the parasphenoid has started to ossify (specimen not figured). It is represented by a faint patch of stained bone in the midline region just anterior to the otic capsules (Figure 3.5G-I).

All specimens at stage 47 now have consistently present dentaries in the lower jaw. The dentary is present as a thin sliver of bone on the anterior margin of Meckel's cartilage (Figure $3.5 \mathrm{G}-\mathrm{I}$ ). The gap between the dentaries (the future symphysis) is wide, as in the one specimen, at stage 46 , with dentaries present. The dentary is slightly bowed with minimal ossification progressing posterolaterally onto the lateral surface of the lower jaw (Figure Figure 3.5G-I). There is no overlap with the coronoid at this stage (Figure 3.5GI). At least one specimen has two to three teeth in the dentary. The coronoid ossification is growing, and it now fills the space between the end of the dentary and the anterior margin of the eye. There are five to seven teeth in each coronoid. 
In the axial skeleton, the vertebral elements have started to ossify in the same order in which they formed, ossifying in the anterior region first and continuing in a posterior direction (data not shown).

Stage 48

The premaxilla has appeared as a thin strip of bone at the anterior margin of the skull (Figure 3.5J-L). A weakly-developed point on its dorsal margin represents the future alary process in one specimen. There are no teeth on the premaxilla at this stage. The vomer and palatine have increased in size slightly. The vomer now appears more oval-shaped than the circular palatine (Figure 3.5J-L). The parasphenoid is now present consistently as a midline patch of triangular- to diamond- shaped bone, just anterior to the otic capsules (Figure 3.5J-L). In one specimen, the parasphenoid has developed a small patch of ossification extending posteriorly between the otic capsules.

In the lower jaw, the dentary is increasing in anteroposterior extent. It now curves onto the lateral surface and occupies approximately one-third of the length of Meckel's cartilage (Figure 3.5J-L). At its posterior extent, it is now over-lapping half of the coronoid laterally. There are teeth in the anterior portion of the dentary of one specimen. Posterior and medial to the dentary, the coronoid is getting larger. It now bears at least seven teeth and appears as a more oval-shaped bone.

\section{Stage 49}

By stage 49 , the internasal plate of the chondrocranium has developed into a robust element in the anterior region of the head (Figure 3.4E, F). It is a thick U-shaped element. In the posterior region of the head, the parachordal cartilage is variable in length at this stage. In some specimens, it continues to extend in a posterior direction, and now extends 
past the posterior limit of the otic capsules to join with the occipital arch (Figure 3.4E, F). In other specimens, it has not yet reached the occipital arch.

The premaxilla now has a definitive $\mathrm{T}$-shape, owing to the thin, dorsally directed alary process (Figure $3.5 \mathrm{M}-\mathrm{O}$ ). The alary process is still relatively short, extending to about the level of the anterior portion of the vomer. In one specimen, the frontals are present as thin slivers of bone, just medial to the eyes. The squamosal is now present as a thin sliver of ossification in the posterodorsal portion of the future element (Figure 3.5M-O). The vomer and palatine are overall similar to those of stage 48 . The vomer is slightly larger and more elongate or bean-shaped than in earlier stages. The pterygoid is now present as a thin process extending posteriolaterally from the palatine, ventral to the eyes. No suture can be seen between the palatine and pterygoid, and so together form the compound palatopterygoid element. The parasphenoid is now an extensive, rectangular-shaped element with a consistently present triangular-shaped posterior portion between the otic capsules (Figure 3.5M-O). In smaller specimens, the parasphenoid terminates anteriorly at the level of the palatine portion of the palatopterygoid; however, in one specimen, it extends a bit further anteriorly to reach the level of the gap between the vomer and palatine (data not shown). At the level of the anterior margin of the otic capsule, the lateral margins of the parasphenoid are incised by the carotid foramina.

In the lower jaw, the dentaries now closely approach one another at the midline symphysis. There are also teeth present in the dentaries for the anterior half of its length. Additionally, the dentary has grown posteriorly to occupy two-thirds of the length of Meckel's cartilage (Figure 3.5M-O). The prearticular has begun ossifying on the medioventral surface of Meckel's cartilage (Figure 3.5M-O). This element has been 
described as a fusion of the prearticular and angular in Ambystoma texanum (Bonebrake and Brandon, 1971); however, separate ossifications indicating a fusion were not observed here in the A. mexicanum specimens. The dentary overlaps the prearcticular ossification for about three quarters of the length of the latter (Figure 3.5M-O). The coronoid is slightly larger with up to ten teeth.

\section{Stage 50}

The parachordal cartilage now consistently articulates with the occipital arch in the posterior region of the head. In terms of ossified elements, the premaxilla is now a strong T-shape with a tall, thin alary process terminating closer to the anterior to the level of the eye than that in stage 49 (Figure 3.6A-C). The alary process is now longer than the ventral tooth bearing portion, which is wide, the latter of which now has five to six teeth present (Figure 3.6A-C). The frontal is present in four out of six specimens. When present, it is a thin sliver of bone representing the lateral margin of the element (Figure 3.6A-C). Anteroposteriorly, the frontal ossifications span the space between where the alary process of the premaxilla terminates and the midpoint of the eye. The squamosal now extends more ventrally as a thin strip of bone that approaches the jaw articulation and is beginning to widen dorsally (Figure 3.6A-C).

On the palate, the vomers and palatines are slightly larger than in stage 49 . The pterygoid is now much longer, reaching the level of just posterior to the eye (Figure 3.6A$\mathrm{C}$, under the right eye). The parasphenoid now consistently extends anteriorly to the level of the gap between the vomer and palatine. The posterior expansion of the parasphenoid, located dorsal to the indentations for the carotid foramina, is now slightly larger and more 
posteriorly expanded. There is a deep incision in the posterior portion of the parasphenoid that receives the notochord (Figure 3.6A-C).

In the lower jaw, the dentary is longer than in stage 49. The prearticular is also now more extensive, where its anterior extent reaches the level of the dentition in the dentary and its posterior extent approaches the terminus of Meckel's cartilage (Figure 3.6A-C). The prearticular is also becoming a wider element posteriorly, giving it a slight wedge shape. The coronoid is slightly larger as well, where it now extends posteriorly beyond the level of the anterior edge of the eye. There are upwards of twelve teeth on the coronoid.

\section{Stage 51}

All specimens at this stage have frontals (Figure 3.6E). Although the anterior limit of the frontal remains roughly the same as that observed in stage 50, the posterior limit of the frontal now extends to the level of the posterior one-third of the eye. In the most advanced specimens, the frontal has started to ossify medially toward the midline, where the two elements will eventually meet. Additionally, ossification of the squamosal is expanding anteriorly in its dorsal portion, giving the anterior margin of this element a pointed outline and generally triangular appearance in this region (Figure 3.6D-F).

The pterygoid portion of the palatopterygoid is now a more robust element than that observed in stage 50, although the amount of ossification in this posterior process differs between specimens. The vomer is slightly more oval, where its anterior margin is growing anteromedially (Figure 3.6D-F). The palatine appears the same as in stage 50. In three specimens, the parasphenoid has extended anteriorly and now terminates at a level between the vomers (Figure 3.6D-F). 
The dentary now terminates almost at the level of the termination of the prearticular (Figure 3.6D-F). The prearticular has increased in ossification and appears more robust, especially at its posterior end. The coronoid is also slightly bigger, as it has increased in size along its anterior-posterior axis.

\section{Stage 52}

Stage 52 is variable with respect to ossification in the skull; however, it is consistent in that the cartilaginous exoccipitals have reached the posterior margin of the otic capsules. Two of the four specimens observed appear identical to specimens described in stage 51, whereas one of the four specimens has a larger frontal and the squamosal has more extensive anterior growth in the dorsal region. The fourth specimen is slightly more advanced yet. There, the alary process of the premaxilla is longer again and is now overlapping the frontal for half of the length of the latter. The frontal has also extended posteriorly and now reaches the posterior margin of the eye (Figure $3.6 \mathrm{G}-\mathrm{I}$ ). The parietal has begun ossification at its lateral margin in this advanced specimen. The thin strip of bone representing the parietal extends from the level of the midpoint of the eye to almost the midpoint of the otic capsule.

On the palate, the vomer is similar in shape and size to that in stage 51 . The palatine portion of the palatopterygoid is now slightly more oval-shaped than before. The pterygoid is wider than it was in stage 51 . The parasphenoid now consistently terminates at the level in between the vomers.

The elements of the lower jaw appear similar in degree of ossification to those in stage 51 . 


\section{Stage 53}

The premaxilla is similar in appearance to that in stage 52 (Figure 3.6G-I). The frontal is now more extensive, reaching the level of the posterior limit of the eye and with more ossification medially. The maxilla is variably present as a thin strip of bone on either one side of the skull only or on both sides. When present, it is a small, narrow element that occurs posterolateral to the lateral most extent of the premaxilla, in the space between the premaxilla and eye. By stage 53, the parietals are consistently present as ossifications in their lateral margins in all specimens (Figure 3.6J-L). They span the space from the midpoint of the eye to the anterior portion of the otic capsule, slightly overlapping the frontals laterally. In three specimens, the dorsal portion of the squamosal is more triangular in shape than in earlier stages, as it expands ossification anteriorly over the lateral surface of the otic capsule. In one specimen, a tiny sliver of bone on the anterior margin of the ventral tip of the squamosal represents the first signs of quadrate ossification.

On the palate, the posterior process of the palatopterygoid continues to ossify posteriorly and has now reached beyond the level as the posterior terminus of the lower jaw (Figure 3.6J-L). It is also much more robust now, posteriorly. The parasphenoid extends anteriorly to occupy much of the space between the vomers (Figure 3.6J-L). Posteriorly, ossification of the parasphenoid reaches the posterior half of the otic capsules. It is still deeply incised by the notochord.

The elements of the lower jaw are much more robust than in stage 52. The dentary now overlaps most of the prearticular and tapers rapidly to a blunter posterior terminus than before. The prearticular is also more robust with a deeper posterior terminus at the jaw articulation. 


\section{Stage 54}

The maxilla is now consistently present on both sides of the skull and has between one and four teeth (Figure 3.7A-C). Both the frontal and the parietal have expanded ossification medially, resulting in wider elements than observed in earlier stages (Figure 3.7A-C). The parietal has grown anteriorly to extensively underlap the posterior portion of the frontal to the level just anterior to the midpoint of the eye. There is still a modest gap between paired right and left frontals and parietals. The squamosal continues to expand into a broad sheet of bone covering the lateral surface of the otic capsule with ossification more extensive than that observed in stage 53 (Figure 3.7A-C). The squamosal is also much broader anteroposteriorly than previously. Both quadrates are consistently present at this stage (Figure 3.7A-C). The element is still represented by a tiny sliver of bone on the anterior margin of the ventral most tip of the squamosal. In the occipital region, the exoccipital has begun ossifying (Figure 3.7A-C). It appears as a thin ossification covering the lateral surface of the cartilage of the occipital condyle.

The vomer has become even more elongated elements on the palate. The palatopterygoid now consistently extends posteriorly beyond the limit of the eye and the posterior portion is wider than that observed in previous stages (Figure 3.7A-C). The lateral margins of the anterior portion of the parasphenoid have grown to a level close to the anterior extent of the vomers (Figure 3.7A-C). Posteriorly, the parasphenoid is slightly more extensive than that in stage 53 .

In the lower jaw, the coronoid appears to be more elongated and the prearticular is becoming more distinctly wedge-shaped as it grows in height at its posterior end. 


\section{Stage 55}

The frontal now tapers to a fine point anteriorly, laterally overlapping the alary process of the premaxilla (Figure 3.7D-F). The maxilla has grown slightly in length and consistently has three or more teeth. The frontal and parietal are more extensive medially but still do not meet at the midline (Figure 3.7D-F). The squamosal is no longer triangular in shape in its dorsal region owing to expanded ossification of the entire element (Figure 3.7D-F). The exoccipital is more extensive and has now ossified medially to encircle the entire occipital condyle cartilage with bone (Figure 3.7D-F).

On the palatal surface, the palatine portion of the palatopterygoid is now more diamond-shaped in outline. The pterygoid portion of the palatopterygoid has continued to extend posteriorly, and in two specimens, it extends slightly beyond the posterior limit of the prearticular. The posterior portion of the parasphenoid, which is positioned between the otic capsules, is now more rectangular in shape than the diamond-like shape observed in previous stages (Figure 3.7D-F). The entire element is also now more robust overall, and in most specimens, completely encloses the carotid foramina.

In the lower jaw, the prearticular is a stronger wedge-shape, with a greater posterodorsal expansion and it is pierced by a tiny foramen posterodorsally.

\section{Stage 56}

By stage 56, the cartilaginous internasal plate has fully formed, still with a distinctive notch in the anterior region, but also with notches in the lateral margins for the olfactory organs (Figure 3.4G, H). Posteriorly, the parachordal plates have merged along the midline of the head at a level that is approximately in the middle of the otic capsules (Figure $3.4 \mathrm{G}, \mathrm{H}$ ). 
Stages 55 and 56 are very similar in terms of their ossifications. Some elements appear less porous than before, suggesting more extensive ossification. The quadrate is slightly larger with a taller dorsoventral extent. The posterior limit of the parasphenoid has also expanded (Figure 3.7G-I). In the lower jaw, the coronoid is now positioned in a trough that is in the dorsal margin of the prearticular.

\section{Stage 57}

By stage 57, the frontal and parietal elements have almost met along the midline of the skull (Figure 3.7J-L). The frontal elements are more closely converging at the posterior portion of the element, whereas the parietals are converging from their midpoint moving posteriorly. The parietal also has a medial extension that extends posteriorly and a lateral process that is approaching the posterior portion of the squamosal (Figure 3.7J-L). The bone on the dorsal portion of the squamosal is less porous than in previous stages, indicating further ossification. On the ventral surface of the parasphenoid, the posterior portion is notched.

In the largest stage 57 specimen, the prefrontal is present (Figure 3.7J-L). It is represented by a tiny dot of ossification at this stage. 
A

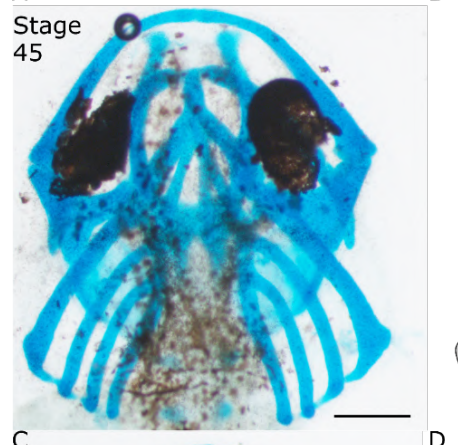

C
Stage
47

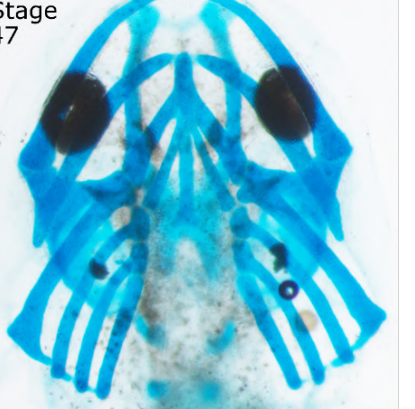

E
Stage

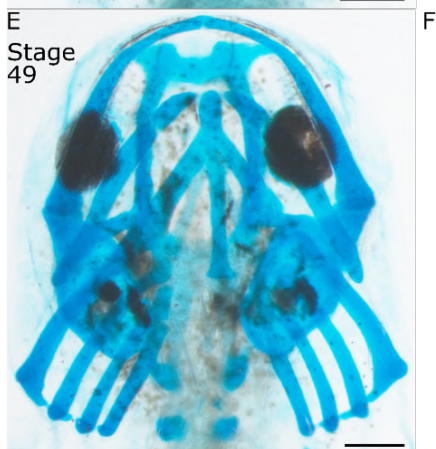

G
Stage
56

$B$

$\mathrm{H}$
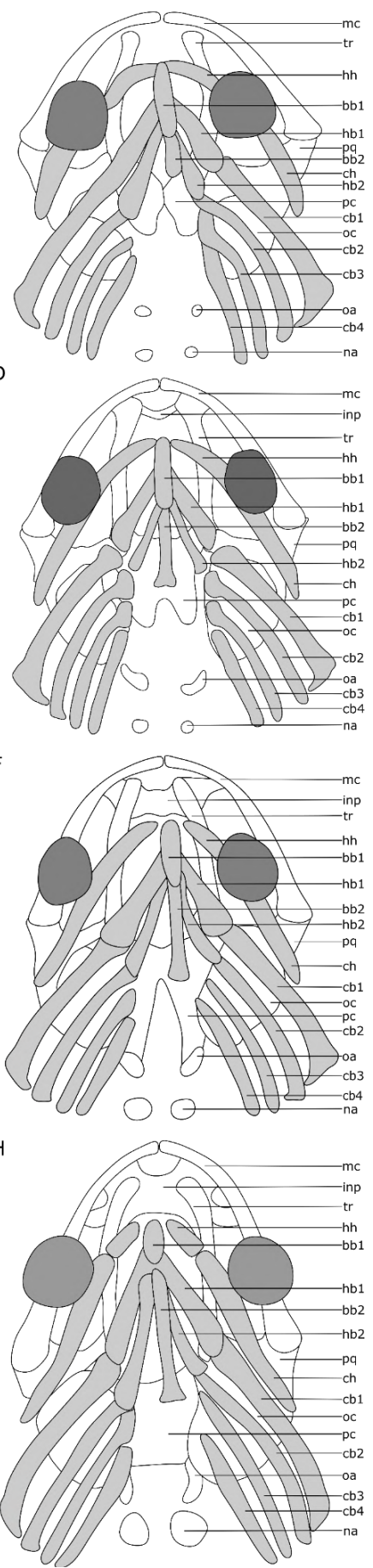

Figure 3.4. Stages 45, 47, 49, and 56, A. mexicanum cartilaginous skull. From left to right, each row consists of a photograph and an illustration in the ventral view. A-B, stage 45; C-D, stage 47; E-F, stage 49; and G-H, stage 56. The hyobranchial apparatus is shown in light grey while other cartilaginous elements are shown in white. Scale bars equal $1 \mathrm{~mm}$. Abbreviations: bb, basibranchial; cb, ceratobranchial; ch, ceratohyal; hb, hypobranchial; hh, hypohyale; inp, internasal plate; mc, Meckel's cartilage; na, neural arches of the vertebral elements; oa, occipital arch; oc, otic capsule; pc, parachordal cartilage; pq, palatoquadrate cartilage; tr, trabecular cartilage. 


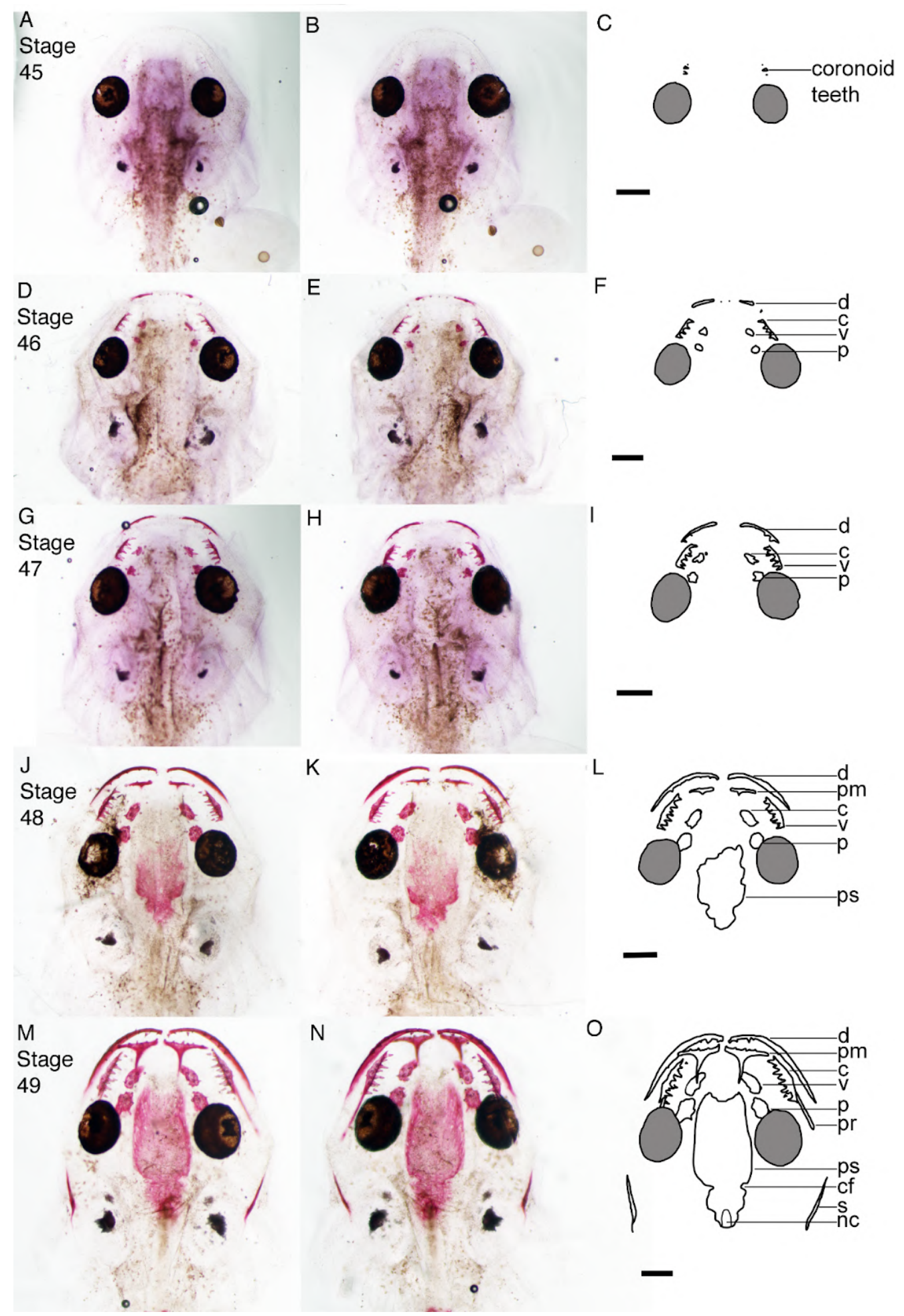

Figure 3.5. Stages 45 to 49 , A. mexicanum ossified skull. From left to right, each row consists of a photograph in dorsal and ventral view, and an illustration in dorsal view. AC, stage 45; D-F, stage 46; G-I, stage 47; J-L, stage 48; and M-O, stage 49. Scale bars equal $1 \mathrm{~mm}$. Abbreviations: c, coronoid; cf, carotid foramen; d, dentary; nc, notochord; pm, premaxilla; p, palatine; pr, prearticular; ps, parasphenoid; s, squamosal; v, vomer. 


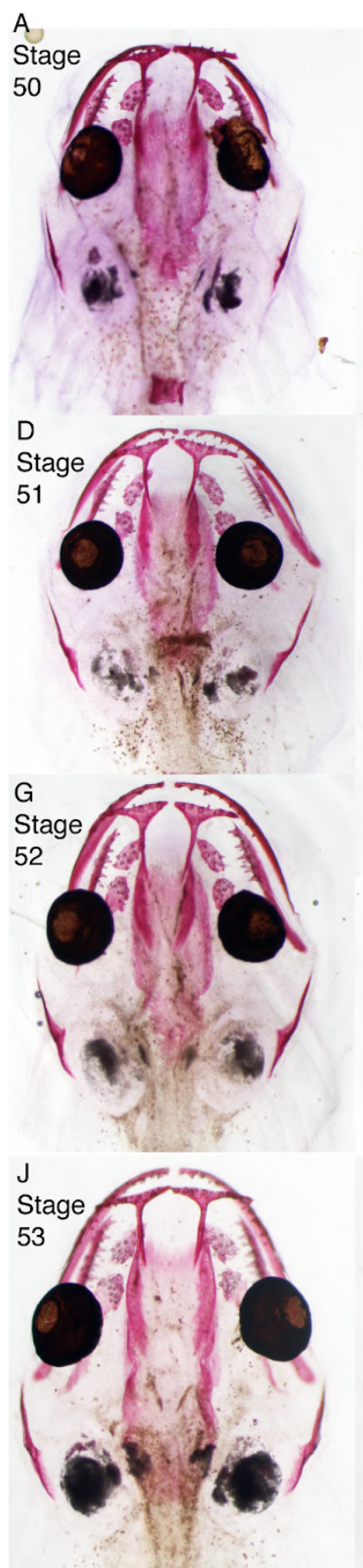

B

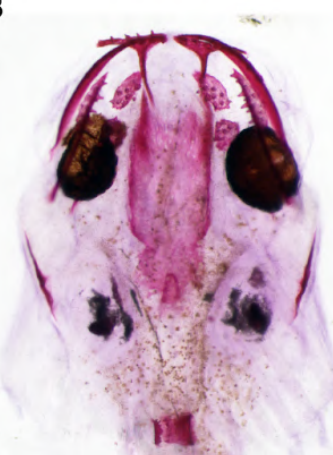

C

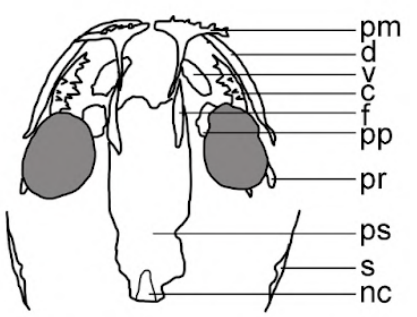

E

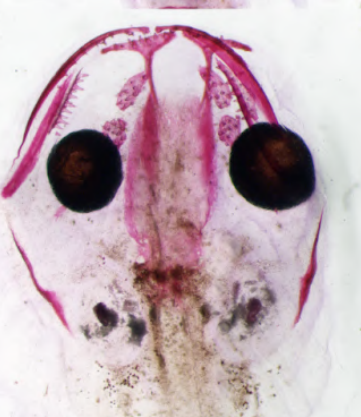

$\mathrm{F}$

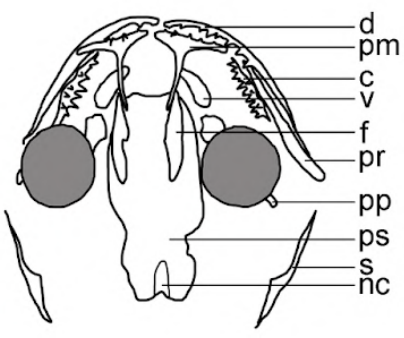

$\mathrm{H}$
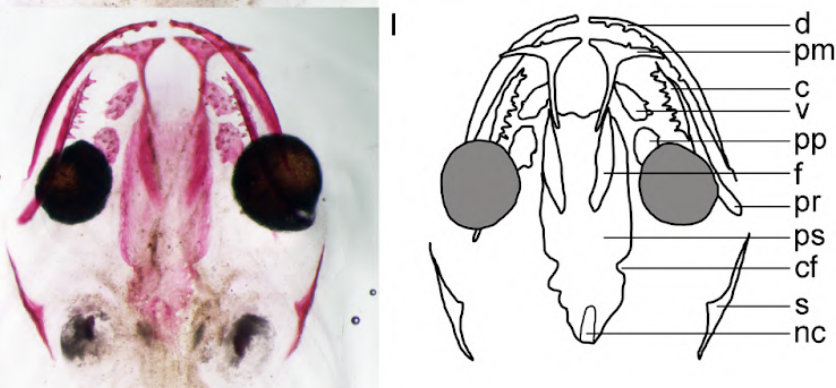

$\mathrm{K}$
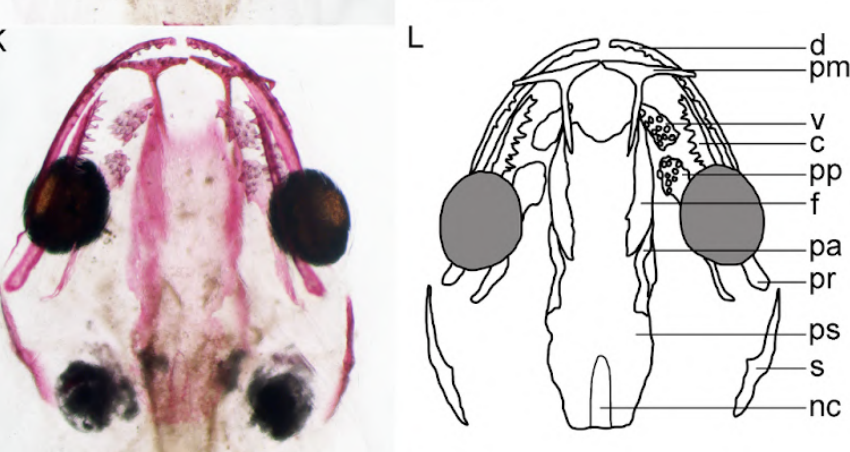

Figure 3.6. Stages 50 to 53, A mexicanum ossified skull. From left to right, each row consists of a photograph in dorsal and ventral view, and an illustration in dorsal view. AC, stage 50; D-F, stage 51; G-I, stage 52; and J-L, stage 53. Scale bars equal $1 \mathrm{~mm}$. Abbreviations: c, coronoid; cf, carotid foramen; d, dentary; f, frontal; nc, notochord; pa, parietal; pm, premaxilla; pp, palatopterygoid; pr, prearticular; ps, parasphenoid; q, quadrate; s, squamosal; v, vomer. 
A
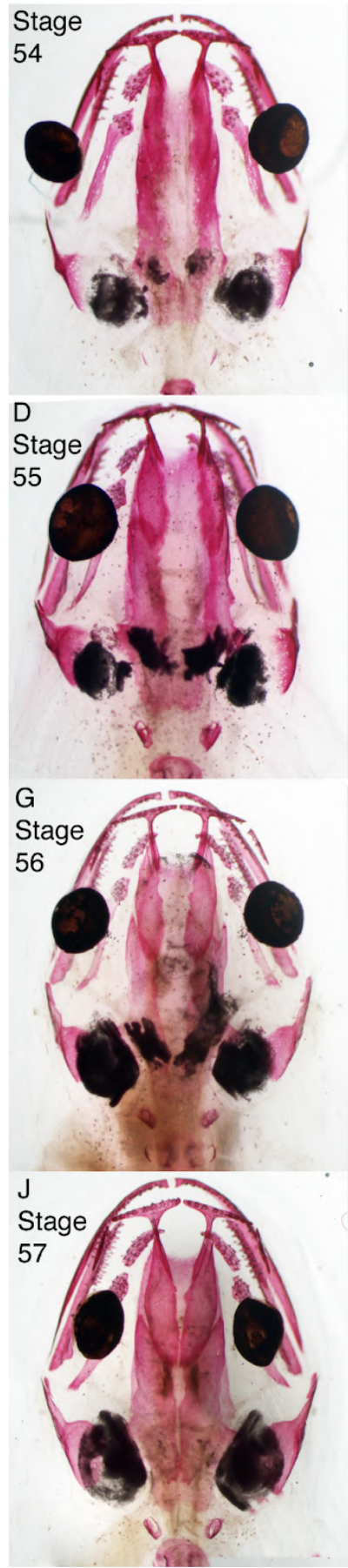

B

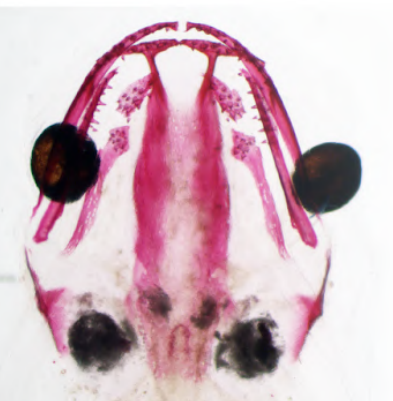

E

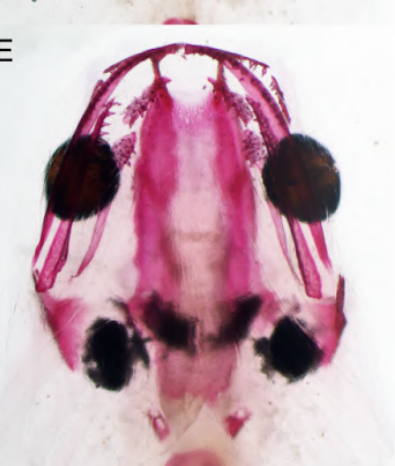

H

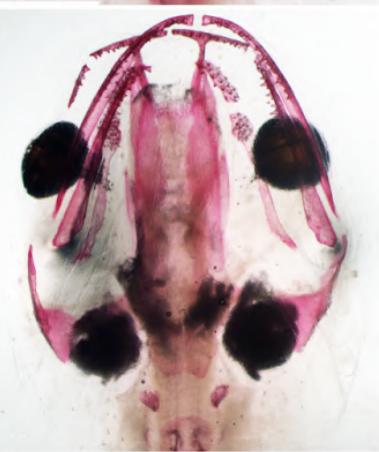

K

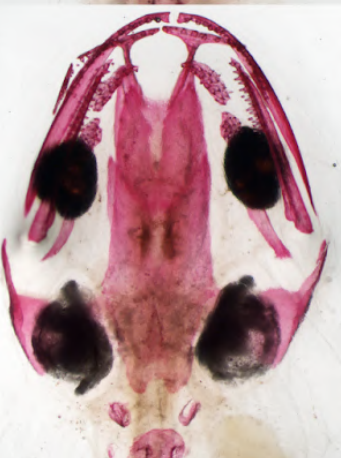

C

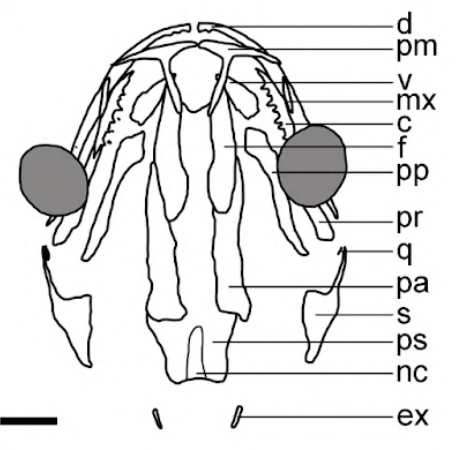

$\mathrm{F}$

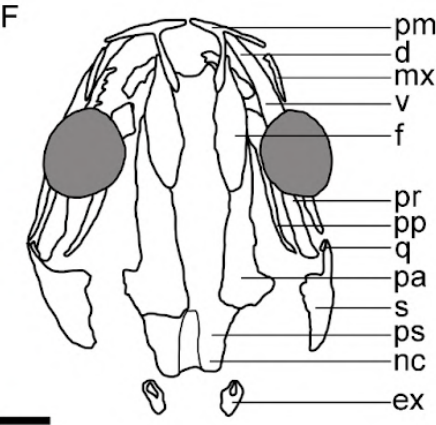

I

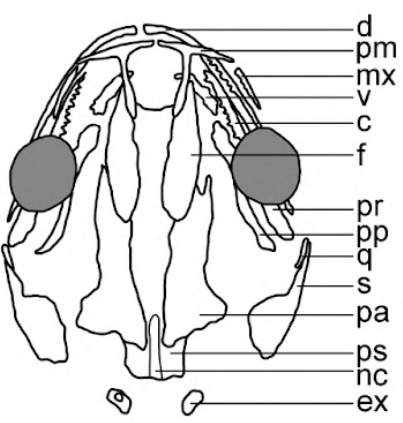

L

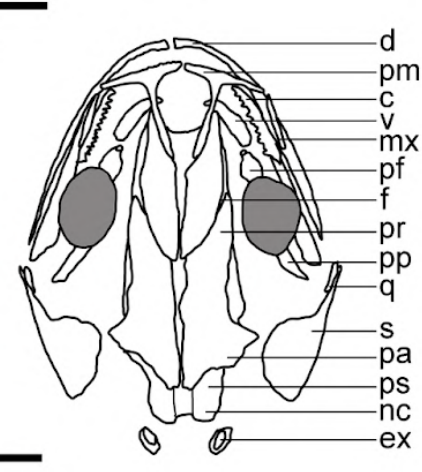

Figure 3.7. Stages 54 to 57 , A. mexicanum ossified skull. From left to right, each row consists of a photograph in dorsal and ventral view, and an illustration in dorsal view. A$\mathrm{C}$, stage 54; D-F, stage 55; G-I stage 56; and J-L, stage 57. Scale bars equal $1 \mathrm{~mm}$. Abbreviations: c, coronoid; d, dentary; ex, exoccipital; f, frontal; mx, maxilla; nc, notochord; pa, parietal; pf, prefrontal; pm, premaxilla; pp, palatopterygoid; pr, prearticular; ps, parasphenoid; q, quadrate; s, squamosal; v, vomer. 


\subsection{3: Xenopus laevis normal skull development: A complementary staging table}

For consistency, the stages below are descriptions of the skull development at stages already established based on primarily external features by Nieuwkoop and Faber (1994). I use the same stage numbers in order to easily integrate the skull development observed here with the most popular and widely used staging table for $X$. laevis. Description of specimens begins at stage 41. This is the first stage when all of the cartilaginous components of the cranium are present (i.e., first appearance of the final structure to chondrify, the occipital arch [Lukas and Olsson, 2018]). At stage 54, ossification begins and, thus, from stage 54 onward, each stage is described based on the state and morphology of the ossifications only. Studied specimens are numbered and listed in Appendix F.

\section{$\underline{\text { Stages } 41 \text { and } 42}$}

Stage 41 and 42 specimens are very similar in degree of development of the chondrocranium and so are described here together. At these stages, the suprarostral plate is the dominant anterodorsal feature of the chondrocranium (Figure 3.8A-C). In pipid species, like $X$. laevis, the suprarostral plate is one large, unpaired structure that is composed of the trabecular cartilages, the ethmoid plate (also called the anterior process of the ethmoid plate by some authors [e.g., de Sá and Swart, 1999]) and the suprarostral cartilage (e.g., Trueb and Hanken, 1992; de Sá and Swart, 1999; Lukas and Olsson, 2017). Therefore, while other cartilages like the palatoquadrate, the subocular, and the parachordal cartilages initially form as discrete structures and later merge with the suprarostral plate, the trabecular, ethmoid, and suprarostral cartilages do not start their development as separate elements in X. laevis (Lukas and Olsson, 2017). 
The anteriormost margin of the suprarostral plate is incised deeply by what will later become the nasal foramina. Along the lateral margins of the anterior portion of the suprarostral plate is the larval pars articularis of the palatoquadrate, which articulates with Meckel's cartilage (Figure 3.8A-C). Two pairs of processes extend posteriorly from the suprarostral plate (Figure 3.8A-C). The lateral pair are the subocular cartilages, and slightly posterior and lateral to the subocular cartilages is a separate pair of cartilaginous elements (Figure 3.8A-C). These elements are the ascending processes of the palatoquadrate. The medial pair of posterior extensions are the trabecular cartilages, which are continuous posteriorly with the parachordal cartilages (Figure 3.8A-C) and between them is a large fenestra that is open posteriorly. This is the basicranial fenestra. The trabecular plus parachordal cartilage processes extend to a level that is either anterior to or between the otic capsules (Figure 3.8A-C). The otic capsule is not attached to other cartilaginous elements at this early stage of development (Figure 3.8A-C). The posteriormost element of the chondrocranium at these stages is the occipital arch. Here the occipital arch appears as a pair of ovoid bodies of cartilage located posterior to the posterior tip of the parachordals (Figure 3.8A-C).

The ventral view of the skull is dominated by the large hyobranchial apparatus; however, just anterior to the hyobranchial apparatus are the three elements of the lower jaw (Figure 3.8A-C). The infrarostral cartilage has started chondrification and is faintly present at the midline (Figure 3.8A-C). It is the anteriormost element. Meckel's cartilage occurs lateral to the infrarostral cartilage. Meckel's cartilage curves laterally and posteriorly, where it meets the palatoquadrate (Figure 3.8A-C). With respect to the hyobranchial apparatus, the anteriormost elements are the paired ceratohyals (Figure 3.8A-C). The 
ceratohyal is a broad plate that curves posterolaterally. The medial portion of the ceratohyal also curves posteriorly and towards the ventral surface (Figure 3.8A-C). Between the ceratohyals is the basihyobranchial. This teardrop shaped element sits posterior to the ceratohyals (Figure 3.8A-C). The branchial basket occurs posterior to the ceratohyals and basihyobranchial and consists of the planum hypobranchiale and the four ceratobranchials (Figure $3.8 \mathrm{~A}-\mathrm{C}$ ). From anterior-posterior, within the branchial basket, is the planum hypobranchiale, which is a broad plate, and the first through fourth ceratobranchials (Figure 3.8A-C). Along the distal and posterior edge of the branchial basket is the commissura terminalis, which connects all four ceratobranchial elements distally. Between each ceratobranchial element are the open pharyngeal clefts.

\section{Stages 43 and 44}

Specimens at stages 43 and 44 are also described together here, as the degree of development of the chondrocranium is largely indistinguishable between specimens at these stages. In the anterior and lateral portion of the suprarostral plate, the incisions for the nasal foramina have become partially closed anteriorly by extensions of the suprarostral plate, giving them the appearance of deepening (Figure 3.8D-F). On the posterior margin of the suprarostral plate, the subocular cartilage has now merged with the ascending process of the palatoquadrate (Figure 3.8D-F). This process of the palatoquadrate is also now longer and more robust. This is the first stage where the subocular fenestra may appear complete but is variable among the specimens examined here (e.g., the subocular fenestra is complete on both sides of the chondrocranium in two specimens, is complete on one side only in three specimens, and has not yet closed on either side in two specimens). When 
present, the subocular fenestra occurs between the subocular cartilage and the trabecular cartilage (Figure 3.8D-F).

Posteriorly, the two parachordal elements have met along the midline in two specimens, thereby completing the basicranial fenestra (Figure 3.8D-F). Posterior to the basicranial fenestra, the parachordal cartilages remain separated from one another, on either side of the notochord (Figure 3.8D-F). The parachordal cartilage has now merged with the occipital arch in three of the seven specimens examined, creating a long process that extends to just posterior to the otic capsule (Figure 3.8D-F). The otic capsule is more robust at stages 43 and 44 than the previous stages, and now has grown closer to the lateral edge of the parachordal cartilage (Figure 3.8D-F).

In the lower jaw, the infrarostral cartilage has made contact with Meckel's cartilage on each side. Meckel's cartilage is also now more elongate and curved posterolaterally (Figure 3.8D-F).

On the ventral surface, the ceratohyal has developed a more prominent, posteriorly directed point at its lateral limit, but otherwise the hyobranchial apparatus appears relatively similar to that of the previous stage (Figure 3.8D-F).

\section{Stages 45 and 46}

By stages 45 and 46, all of the various cartilaginous elements on the dorsal surface are now connected to one another in every specimen, including the otic capsule, which was previously separated from the other cartilaginous elements of the chondrocranium (Figure 3.8). In the anterior region of the dorsal surface, the nasal foramina are complete in all specimens by stage 45 . The foramina are bordered anteriorly, medially and posteriorly by the suprarostral plate and laterally by the quadratoethmoidal, an extension of the 
palatoquadrate pars articularis (Figure 3.8G-I). The quadratoethmoidal and the anterior projection of the suprarostral plate meet anteriorly to form the base of the tentacular cartilage. In some specimens at these stages, the tentacular cartilage already has an anterior projection (e.g., Figure 3.8I). The ascending process of the palatoquadrate has once again changed shape, it has grown longer along its proximal to distal axis. In two specimens, the ventrolateral process of the palatoquadrate is present (Figure 3.8I). This process is attached to the distal end of the ascending process of the palatoquadrate.

Along the midline of the skull in five out of ten specimens, the frontoparietal fontanelle is now defined. This fontanelle represents the space that will accommodate the future frontoparietal on the skull roof (Figure 3.8I, midline). It is bordered anteriorly by the suprarostral plate, and on either side by the trabecular cartilage. The margins of the basicranial fenestra are now closed in all specimens examined, but the size of the fenestra varies among specimens. In three specimens there are paired carotid foramina on either side of the basicranial fenestra that accommodate the carotid arteries (Figure 3.8G-I [Lukas and Olsson, 2017]). Posterior to the basicranial fenestra, the each parachordal cartilage remains separated by the notochord. In every specimen, the parachordal and occipital arch have met and in one specimen the metotic fissure, a gap between the occipital arch and otic capsule, has closed to form the metotic foramen, also referred to as the exoccipital foramen (Figure 3.8I [Clack, 2002; Naumann and Olsson, 2017; Quinzio and Fabrezi, 2018]). This is the foramen that transmits cranial nerve $\mathrm{X}$ (Naumann and Olsson, 2017; Quinzio and Fabrezi, 2019). The otic capsule is now attached to the rest of the chondrocranium via the trabecular cartilage dorsally and the parachordal cartilage ventrally (Figure 3.8G-I). 
The elements of the lower jaw are more robust, but not significantly different in shape from the previous stages. The ceratohyal has become more robust and this paired element more closely approaches its mate at the midline. The basihyobranchial now sits in a groove between the two ceratohyals. In five of the ten specimens examined, the branchial basket has elongated along the anterior-posterior axis, and now is more oval in shape instead of being more circular (Figure 3.8G-I).

\section{Stages 47 and 48}

At stages 47 and 48, the anterior portion of the suprarostral plate is overall similar in morphology to that of the previous stages (Figure 3.8J-L). By now, the ventrolateral process of the palatoquadrate is consistently present in all specimens (Figure $3.8 \mathrm{~J}-\mathrm{L}$ ). The basicranial fenestra continues to be variable in size, but in most specimens, it has shrunk in size to around $250 \mu \mathrm{m}$ long and $100 \mu \mathrm{m}$ wide, situated between the trabecular cartilages and visible dorsally through the frontoparietal fontanelle (Figure 3.8J-L). The exoccipital foramen is consistently present in all specimens now (Figure 3.8J-L). Additionally, there is a small cartilaginous process coming off the posterolateral corner of the otic capsule. This is the muscular process of the otic capsule.

The structures of the lower jaw and hyobranchial apparatus are similar in degree of development to those of the previous stages (Figure 3.8J-L).

\section{Stage 49}

At stage 49, the tentacular cartilage is now consistently present as an anterolaterally projecting point at the apex of the quadratoethmoidal cartilage and the anterior projection of the suprarostral plate (Figure 3.9A-C). In the posterior region of the chondrocranium, 
the occipital arch does not appear to protrude beyond the posterior margin of the otic capsule due to growth of the latter (Figure 3.9A-C).

The only other morphological change that differentiates stage 49 from other stages is that in some specimens the basicranial fenestra has been completely infilled by cartilage. It is no longer present in three of the eight specimens examined (data not shown).

\section{Stages 50 and 51}

In the anterior region of the dorsal chondrocranium, the tentacular cartilage has grown into a tentacle structure, and it is now consistently present but remain short $(1 \mathrm{~mm}$ or shorter in length; Figure 3.9D-F). In the middle of the chondrocranium, the basicranial fenestra has been completely infilled by cartilage in all specimens examined (Figure 3.9DF). The ventrolateral process of the palatoquadrate is much larger and more prominent than in previous stages, projecting both anteriorly and posteriorly at its distal end (Figure 3.9DF). In the otic capsule, the epiotic eminence is visible for the first time, as well as the larval crista parotica. The larval crista parotica is faint and does not appear in images as it is obscured by the otic capsule; however, when manually manipulating specimens, it can be seen located lateral and ventral to the otic capsule.

Cartilaginous neural arches are present in the axial skeleton by stages 50 and 51 (Figure 3.9D-F).

\section{Stages 52 and 53}

Stages 52 and 53 are the final stages where only the chondrocranium is present. In general, specimens at stages 52 and 53 are more elongated anteroposteriorly than those of previous stages, which had consistently more rounded heads (Figure 3.9G-I). The tentacular cartilage is now a long structure, consistently longer than $1 \mathrm{~mm}$, which was the 
maximum length observed at stages 50 and 51 (Figure 3.9G-I). The frontoparietal fontenelle, which was present in previous stages, now resembles the shape and size of the future frontoparietal bone. The ascending process of the palatoquadrate has thickened along the anteroposterior axis (Figure 3.9G-I). The protruding lower jaw is more strongly arched than that of previous stages, and the hyobranchial apparatus has expanded posteriorly, contributing to the more elongate shape of the head overall (Figure 3.9G-I).

\section{$\underline{\text { Stages } 54 \text { and } 55}$}

At stages 54 and 55, the first ossifications appear in twelve of the nineteen specimens examined. For the rest of the stages, only ossified elements will be described as they provide a better basis for staging in these older developmental stages than the cartilages. In five of the nineteen specimens examined, only the parasphenoid is present, which makes it the first element to ossify in the skull (specimen not figured). In seven specimens, both the parasphenoid and the frontoparietal is present (Figure 3.10A-C). The frontoparietal has started to ossify on the dorsal surface, with one ossification centre occurring in each element representing its lateral portion (Figure 3.10A-C). Anteriorly, the frontoparietal terminates at approximately the same level as the parasphenoid and posteriorly it terminates anterior to the otic capsule (Figure $3.10 \mathrm{~A}-\mathrm{C}$ ).

On the palatal surface of the skull, the parasphenoid is present as a thin sliver of bone along the midline. Anteriorly, the parasphenoid terminates at a level equal to the midpoint of the eyes and posteriorly the parasphenoid terminates between the otic capsules (Figure 3.10A-C). 


\section{Stage 56}

As in stages 54 and 55, the elements that are present in the skull are the frontoparietal and the parasphenoid. In one specimen, only the parasphenoid is present, whereas in all other specimens examined both the frontoparietal and parasphenoid are present. For most specimens observed, the frontoparietal appears the same as in previous stages. However, in two specimens, the frontoparietal has started to ossify towards the midline of the skull (specimen not figured). On the palatal surface of the skull, in all specimens observed, the parasphenoid has started to widen and lengthen (Figure 3.10DF). The parasphenoid now terminates posteriorly between the two prootic elements. In one specimen, the otic capsule has started to ossify in the ventromedial region of the structure (Figure 3.10D-F). The otic capsule is part of the prootic (Trueb and Haken, 1992). As the otic capsule portion of the prootic makes up a substantial portion of the prootic and the morphology of the otic capsule is an important morphological feature for some stages, the otic capsules are additionally labelled here (e.g., Figure 3.10D-F, po oc). There is no evidence of a discrete opisthotic ossification. In another specimen, ossification of the medial angulosplenial has just begun (this specimen not figured).

In the axial skeleton, ossified vertebral elements are present in all specimens (Figure 3.10D-F). This first appearance of ossification occurs in the anteriormost elements, as ossification proceeds in an anterior (early development) to posterior (later development) direction.

\section{Stage 57}

Specimens at stage 57 are variable in terms of the extent of ossification of various elements; however, it is less variable in terms of which elements have begun to ossify. All 
stage 57 specimens have a parasphenoid, frontoparietal, ossifications in prootic (in the otic capsule portion), exoccipital, and the medial angulosplenial in the lower jaw (Figure 3.10G-I). The frontoparietal fontanelle is visible at this stage as a grey outline along the midline of the skull (Figure 3.10G, H). The ossifications of the frontoparietal within the frontoparietal fontanelle indicate that the frontoparietal has not yet fully ossified anteriorly and posteriorly along the lateral extent (Figure 3.103G-I). However, in most specimens each frontoparietal has started to ossify medially towards its mate (Figure 3.10G-I). On the palatal surface, the parasphenoid now better resembles the stereotypical spear shape typical of X. laevis with a pointed anterior end and blunt posterior end (Figure 3.10G-I). The middle portion is now noticeably wider than the anterior and posterior portions (Figure $3.10 \mathrm{G}-\mathrm{I})$.

In the posterior region of the skull, the level of ossification is variable among specimens at this stage. In the prootic, the otic capsule has begun to ossify in the medial region, forming a bony surface over the medial portion of the anterior and posterior semicircular canals dorsally and the medial portion of the floor of the otic capsule (Figure 3.10G-I). The exoccipital has started to ossify within the occipital arch; they are visible dorsally and ventrally, just posterior to the otic capsules (Figure 3.10G-I). The exoccipital foramen is now surrounded by ossification and is visible on the ventral surface of the exoccipital. This foramen transmits the vagus nerve, cranial nerve X (Naumann and Olsson, 2017; Quinzio and Fabrezi, 2018).

In the lower jaw, one element, the medial angulosplenial is present in all specimens examined. It is a thin line of ossification along the medial surface of Meckel's cartilage (Figure 3.10G-I). 


\section{$\underline{\text { Stages } 58 \text { and } 59}$}

The frontoparietal has almost ossified to its full anterior and posterior extents along the lateral edge of the element and continues to be variably ossified in its medial portion (Figure 3.10J-L). By stage 58, the anterior portion of the frontoparietal appears as a square with rounded edges (Figure $3.10 \mathrm{~J}-\mathrm{L}$ ). The frontoparietal of some specimens has met its mate at the midline anteriorly and together the elements have begun to define the margins of the pineal foramen (specimens not figured). In one stage 59 specimen, the maxilla has just begun ossification in the anterior region of the skull (not visible in figured specimen).

In the posterior region of the skull, the otic capsule is now the dominant ossified elements due to expansion of the prootic ossification (Figure 3.10J-L). Some variation exists in the extent of ossification of the otic capsule. Most specimens have at least the anterior and posterior semicircular canals covered in bone, and a smaller number of specimens now have ossification covering the horizontal semicircular canal (Figure 3.10JL). Outside of the otic capsule portion of the prootic, the prootic has also changed shape medially to the otic capsule and has begun to ossify towards the midline of the skull and towards the anterior portion of the skull (Figure 3.10J-L). Additionally, the portion of the prootic that is not the otic capsule has ossified posteriorly and has started to articulate with the exoccipital (Figure 3.10J-L). When compared to previous stages, the exoccipital has become a wider element (Figure 3.10).

In the lower jaw, the medial angulosplenial is longer than it was in stage 57 (Figure $3.10 \mathrm{~J}-\mathrm{L})$. 


\section{Stage 60}

In one specimen at stage 60 , both paired maxillae and premaxillae are present, whereas in a second specimen both premaxillae are present but only the left maxilla is present. When present, the maxilla and premaxilla are the anteriormost elements (Figure $3.11 \mathrm{~A}-\mathrm{C})$. A row of premaxillary and maxillary teeth is inconsistently present among specimens. When present, they are not yet mineralized at their bases and so appear as floating crowns (Figure 3.11B). This first generation, or series, of teeth typically forms early in metamorphosis (Shaw, 1979). The nasal is variably present at this stage, and when present it is a faint, crescent-shaped ossification that occurs just anterior to the frontoparietal (Figure 3.11A-C). The frontoparietal has now reached its anterior and posterior limit on the lateral edge of the element but is still variably ossified in its medial region (Figure 3.11A-C). In one specimen, the two frontoparietals have nearly met along the midline in the central region of the element (specimen not figured), whereas in the second specimen the frontoparietals have met along the midline in the anterior region of the element (Figure 3.11A-C). Both specimens have now completed ossification around the pineal foramen (Figure 3.11A-C). The posterior portion of the lateral edge of the frontoparietal overlie the medial portion of the otic capsule (Figure 4A).

The otic capsule ossification remains variable in its extent, with one specimen showing complete ossification (specimen not figured), whereas in the other specimen only the area surrounding the semicircular canals is ossified (Figure $3.11 \mathrm{~A}-\mathrm{C}$ ). The medial portion of the otic capsule is more robustly ossified than observed in previous stages but otherwise has the same morphology as in stages 58 and 59, and the exoccipital continues to ossify posteriorly towards it (Figure $3.11 \mathrm{~A}-\mathrm{C}$ ). 
In the lower jaw, the medial angulosplenial is now more extensively ossified posteriorly, reaching the midpoint level of the eye (Figure 3.11A-C).

\section{Stage 61}

The maxilla, premaxilla, and nasal are still variably present elements by stage 61 . The nasal is present in one stage 61 specimen (specimen not figured) but not yet present in a second specimen (Figure 3.11D-F). The row of teeth on the premaxilla and maxilla observed in one stage 60 specimen is not present in either stage 61 specimens, indicating variability in their presence across these two stages. The frontoparietal continues to be variably ossified in the medial portion of this element, identical to morphologies seen in stage 60 (Figure 3.11D-F). The otic capsule portion of the prootic, however, is now consistently fully ossified (Figure 3.11D-F). Each otic capsule has a large foramen visible on the ventrolateral surface; this is the fenestra ovalis (Figure 3.11F). The anterior, posterior, and horizontal semicircular canals are now all covered in bone (Figure 3.11DF). The medial portion of the otic capsule ossification has the same morphology on the dorsal and ventral surfaces as was observed in stages 58 through 60 .

In the lower jaw the medial angulosplenial continues to ossify along the medial surface of Meckel's cartilage. The medial angulosplenial is more robust than that observed in previous stages (Figure 3.11D-F). A second ossification, the lateral angulosplenial, is now variably present (Figure 3.11D-F). The lateral angulosplenial, when present, is a very thin line of bone on the lateral surface of the lower jaw in the posterior region (Figure 3.11D-F). 


\section{Stage 62}

By stage 62, both the premaxilla and maxilla are consistently present. The premaxilla is now roughly $\mathrm{T}$-shaped, with the development of the dorsal alary process (Figure $3.11 \mathrm{G}-\mathrm{I}$ ). The maxilla has ossified medially to the extent that it either nearly articulates (Figure 3.11G-I) or does articulate with the lateral processes of the premaxilla. The tooth row first visible in stage 60 is now consistently present in all specimens and these teeth continue to appear as a row of floating crowns (Figure $3.11 \mathrm{G}-\mathrm{I}$ ). The nasal is still variably present. The septomaxilla is now also variably present and appears on both sides of the skull in one specimen (Figure $3.11 \mathrm{G}-\mathrm{I}$ ), on only the left side of a second specimen, and is not present in a third specimen. When present, the septomaxilla appears as a faint line of ossification that occur just lateral to the anterior portion of the frontoparietal (Figure 3.11G-I). The two frontoparietal elements now meet very closely along the midline (Figure 3.11G-I). They may form a suture in the anterior and posterior regions of the bone; however, the middle portion of the suture is variably present, and a small gap may persist (Figure 3.11G-I). The pineal foramen is consistently defined in all specimens at this stage (Figure 3.11G-I).

Anterior and lateral to the otic capsule is a faint ossification. This is the first appearance of the squamosal (Figure $3.11 \mathrm{G}-\mathrm{I}$ ). The squamosal is present as a thin line of ossification anterolateral to the otic capsule. The medial margin of the prootic has started to ossify towards the midline on both the dorsal and ventral surfaces (Figure 3.11G-I).

In the lower jaw, the medial angulosplenial resembles the morphology of stage 61, whereas the lateral angulosplenial is now consistently present but variable in extent of 
ossification. The lateral angulosplenial is a thin line of ossification on the posterior portion of the lower jaw (Figure 3.11G-I).

\section{Stage 63}

The lateral processes of the premaxilla have extended laterally and are wider than the alary process is tall (Figure $3.11 \mathrm{~J}-\mathrm{L}$ ). The premaxilla and maxilla have now ossified to meet and surround the bases of the teeth observed in previous stages (Figure 3.11J-L). The septomaxilla is now present in all specimens on both sides of the skull, where it can be seen to be a rod-shaped bone. At this stage, the medial and lateral extents of the septomaxilla are well ossified, but ossification in the central portion is variable among specimens (Figure 3.11J-L). In one specimen, there is little ossification in the central portion, whereas in a second specimen the central portion is completely ossified. The nasal is also consistently present and now its posterior portions underlies the anterior portion of the frontoparietal (Figure 3.11J-L).

On the palatal surface, the anterior portion of the parasphenoid comes to a point at a level that is variable between specimens. In one specimen, the anterior limit almost reaches the level of the alary process of the premaxilla (Figure $3.11 \mathrm{~J}-\mathrm{L}$ ), whereas in a second specimen the anterior limit is posterior to the premaxilla. One specimen shows signs of early ossification of the sphenethmoid. The sphenethmoid appears as a very thin line running along the lateral edge of the widest point of the parasphenoid (not visible in the figured specimen). At stage 63, the otic capsule portion of the prootic and the exoccipital have changed shape on their dorsal surface. The medial portion of the otic capsule is much

more extensively ossified than that in stage 62 (Figure 3.11J-L) and the posterior end of the exoccipital now has medially directed flange (Figure $3.11 \mathrm{~J}-\mathrm{L}$ ). 
In the lower jaw, all three elements (dentary, medial and lateral angulosplenial) are now present and variably ossified (Figure $3.11 \mathrm{~J}-\mathrm{L}$ ). Anteriorly, the two halves of the dentary approach one another closely at the midline (Figure 3.11 J-L). Posteriorly, ossification of the dentary reaches the level of the posterior extent of the dorsally located maxilla (Figure 3.11J-L). The largest element, the medial angulosplenial, has an anterior extent that is either approximately level with the midpoint of the dentary or more posterior. Posteriorly, the medial angulosplenial is more extensively ossified and has almost ossified to the level of the posterior extent of the lower jaw (Figure 3.11J-L). The lateral angulosplenial has grown in size along its anterior-posterior axis (Figure 3.11J-L).

\section{Stage 64}

On the ventral surface of the maxilla and premaxilla elements, a second more lingually positioned row of teeth has started to form in the anterior portion of the maxilla in one specimen (Figure 3.12A-C; Figure 3.13). This is the replacement series of teeth that occurs posterior and in between teeth present of the first series, as described by Shaw (1979). The labial series of teeth, the row that was first to develop, are larger than the lingual series (Figure 3.13). The suture between the frontoparietals may be partially obliterated by fusion of the two elements at this stage in the anterior region only (Figure 3.12A-C). The suture is still visible posterior to the pineal foramen in all specimens observed at this stage (Figure 3.12A-C).

On the palatal surface, ossification of the sphenethmoid is only present in one specimen and its morphology resembles that of stage 63 . The pterygoid is now present on the palatal surface (Figure 3.12A-C). This bone is triangular in shape with one point directed anteriorly, and the other two directed posterolaterally and posteromedially, 
respectively (Figure 3.12A-C). In the posterior region of the skull, anterior and lateral to the otic capsule, the squamosal has slightly changed in shape (Figure 3.12A-C). The squamosal is now roughly $\mathrm{C}$-shaped, with the concavity opening posteriorly.

Elements of the lower jaw have increased in their amount of ossification.

Stage 65

On the ventral surface of the maxilla and premaxilla, the two series of teeth are consistently present (Figure 3.12D-F, Figure 3.13). The septomaxilla is broader and more robust in one specimen, where it now appears to be dumbbell shaped (Figure 3.12D-F). The posterior portion of the septomaxilla now underlies the nasal (Figure 3.12D-F). At the anterior and medial extent of the nasal, the paired nasal elements make contact with one another along their midline. The suture between the two frontoparietals may be obliterated by this stage (as seen in one specimen), with a pineal foramen remaining located in the anterior half of the element, at a level approximately equal to the anterior extent of the eyes (Figure 3.12D-F). In the other three specimens, the suture is only obliterated in the anterior portion (specimens not shown, but this is the morphology observed in stage 64 specimens).

On the palatal surface, in the anterior region of the skull, the vomer is now present (Figure 3.12D-F). The vomer appears as a small, unpaired element located ventral to the parasphenoid. The vomer is longer in its medial region, tapering to points at each lateral extent of the element (Figure 3.12D-F). The vomer arises from two ossification centres on the left and right sides of the skull that subsequently fuse at the midline (Trueb and Hanken, 1992). By stage 65, the anterior portion of the parasphenoid consistently almost reaches the level of between the alary processes of the premaxillae (Figure 3.12D-F). This is the complete extent of the parasphenoid will grow anteriorly. On either side of the 
parasphenoid, the very thin lines of ossification that represent the paired sphenethmoid elements have thickened in one specimen (not shown) and are not yet visible in the others (Figure 3.12D-F).

The dorsal end of the squamosal remains pointed, as in previous stages; however, the ventral end has widened to a blunt point (Figure 3.12D-F). The columella (or stapes) is now present for the first time at stage 65 (Figure 3.12D-F). Its proximal end rests adjacent to the fenestra ovalis of the otic capsule, and distally extends anteriorly. Anterior and ventral to the otic capsule, the pterygoid is now a larger, triangular element than observed in previous stages, especially when viewed from the ventral surface (Figure 3.12D-F)

In the lower jaw, the three elements (dentary, medial and lateral angulosplenial) have started to articulate with each other in some specimens. In one specimen, all three elements are articulated on the dorsal surface and in the posterior region of the lower jaw only on the left side (specimen not figured), whereas in a second specimen this morphology is present on both sides of the lower jaw (not shown). In two other specimens, only the medial and lateral angulosplenials have started to articulate on the dorsal surface and the dentary is still a separate element (Figure 3.12D-F).

\section{Stage 66}

Stage 66 is the final stage described by Nieuwkoop and Faber (1994). By this stage, the frog has virtually completed metamorphosis based on external features. The skull, however, has not completely finished development (i.e., the sphenethmoid has not finished ossifying), however, the occiput has finished at this point. 
On the dorsal surface, the suture between the frontoparietals is entirely obliterated at this stage (Figure 3.12G-I). On the palatal surface, on either side of the parasphenoid, the sphenethmoid has ossified laterally and is now visible in all specimens (Figure 3.12GI). This element ossifies lateral and dorsal to the parasphenoid, forming a cup shape in cross-section with its mate and the parasphenoid below. The sphenethmoid is more extensively ossified in its anterior region and has a foramen located approximately midway along its anterior-posterior axis; this is for the optic nerve (Figure 3.12G-I).

Morphological changes in the posterior portion of the skull are extensive. The dorsal portion of the squamosal now has an anteriorly directed process (Figure 3.12G-I). The posterior apex of the triangular pterygoid has expanded such that the pterygoid now underlies the anterior portion of the otic capsule (Figure 3.12G-I).

The elements of the lower jaw do not differ when compared to stage 65 , except that in all specimens the medial and lateral angulosplenial have now articulated in their posterior ends, as first observed in stage 65 (Figure 3.12G-I). 


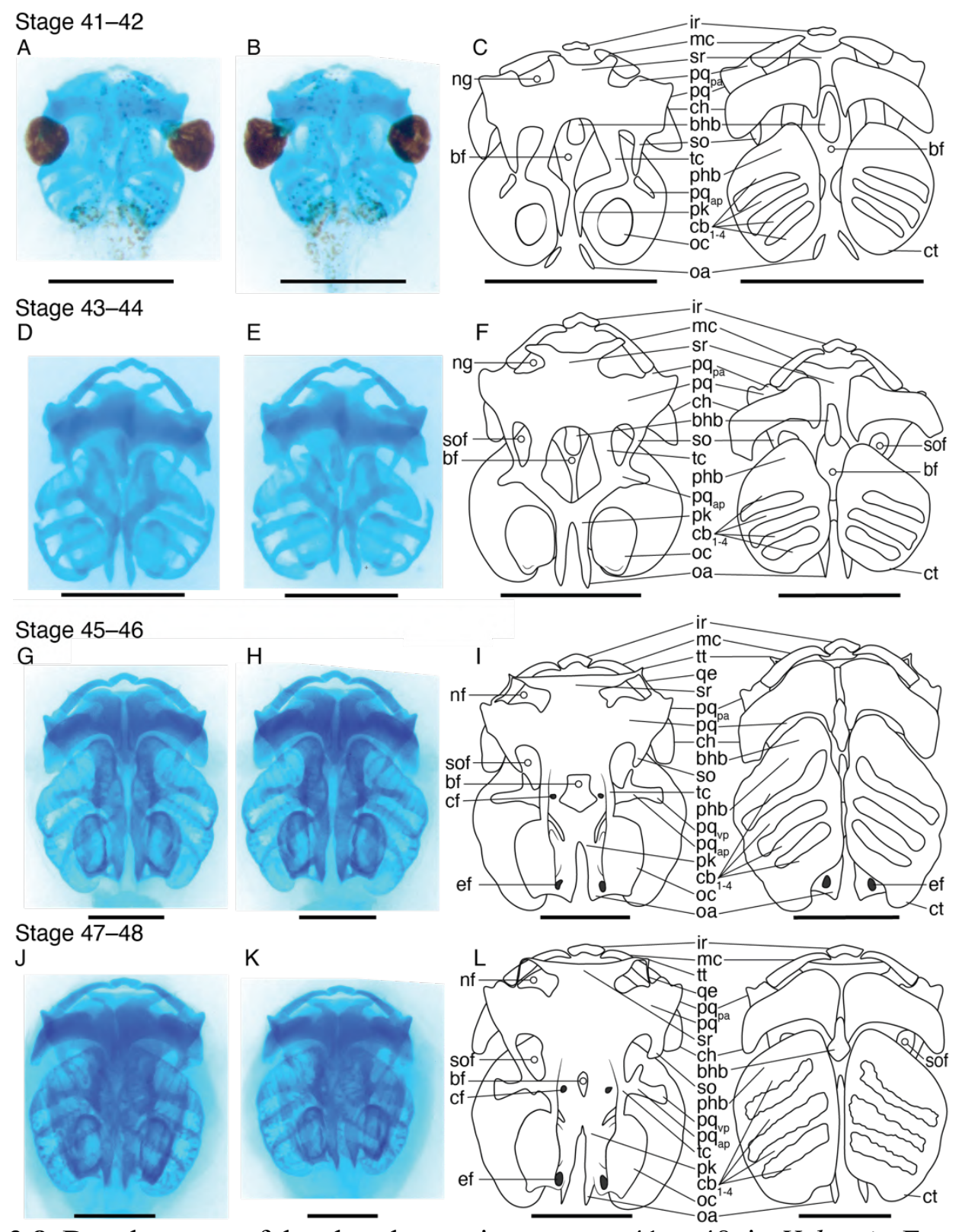

Figure 3.8. Development of the chondrocranium, stages 41 to 48 , in $X$. laevis. From left to right, each row consists of a photograph in dorsal (right) and ventral view (left), with an illustration in dorsal (right) and ventral (left) view. A-C, stages 41-42; D-F, stages 43-44; G-I, stages 45-46; J-L, stages 47-48. Lines to with filled circles indicate small foramina and lines that end with open circles indicate larger foramina. Scale bars equal $1 \mathrm{~mm}$. Abbreviations: bf, basicranial fenestra; bhb, basihyobranchial; $\mathrm{cb}_{1-4}$, ceratobranchials one through four; cf, carotid foramen; ch, ceratohyal; ct, commisura terminalis; ef, exoccipital foramen; ir, infrarostral; mc, Meckel's cartilage; nf, nasal foramen; oa, occipital arch; oc, otic capsule; phb, planum hyobranchiale; pk, parachordal cartilage; pq, palatoquadrate; $\mathrm{pq}_{\mathrm{ap}}$, ascending process of the palatoquadrate; $\mathrm{pq}_{\mathrm{pa}}$, pars articularis of the palatoquadrate; $\mathrm{pq}_{\mathrm{vp}}$, ventrolateral process of the palatoquadrate; qe, quadratoethmoidal; so, subocular cartilage; sof, subocular fenestra; sr, suprarostral plate; tc, trabecular cartilage; tt, tenticular cartilage. 
Stage 49

A

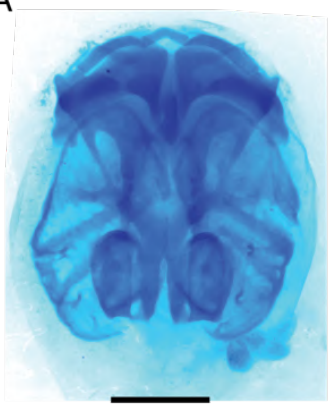

Stage 50-51

D

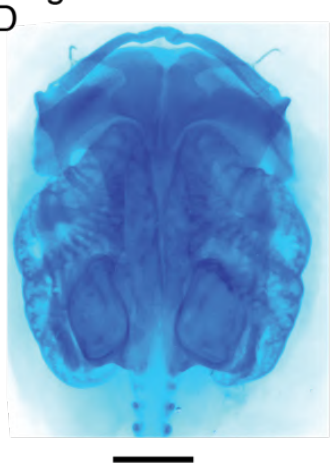

Stage $52-53$

G

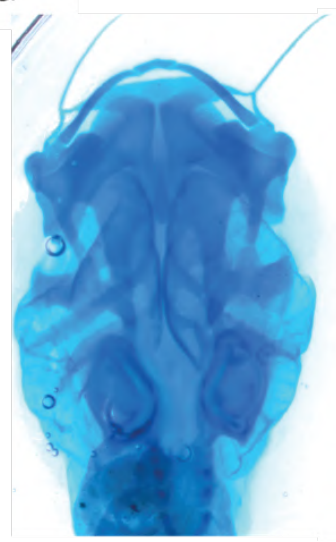

B

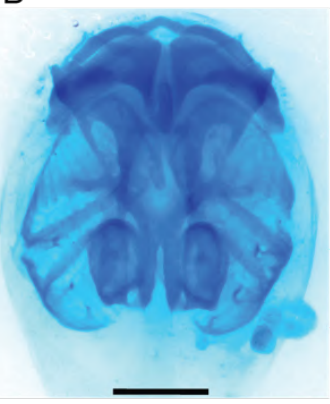

$\mathrm{E}$

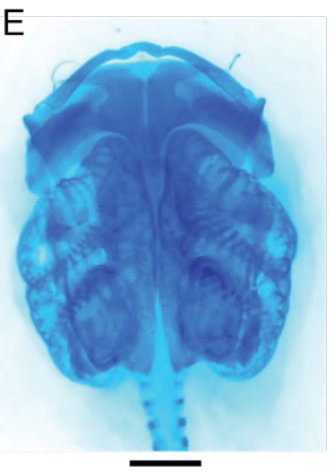

$\mathrm{H}$

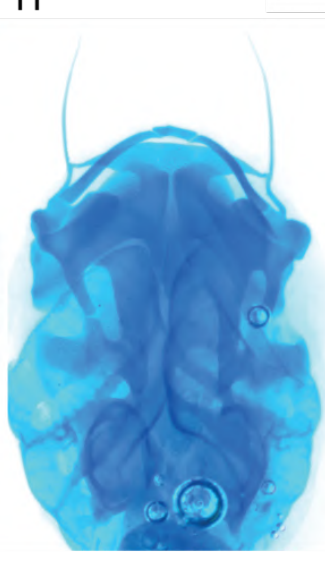

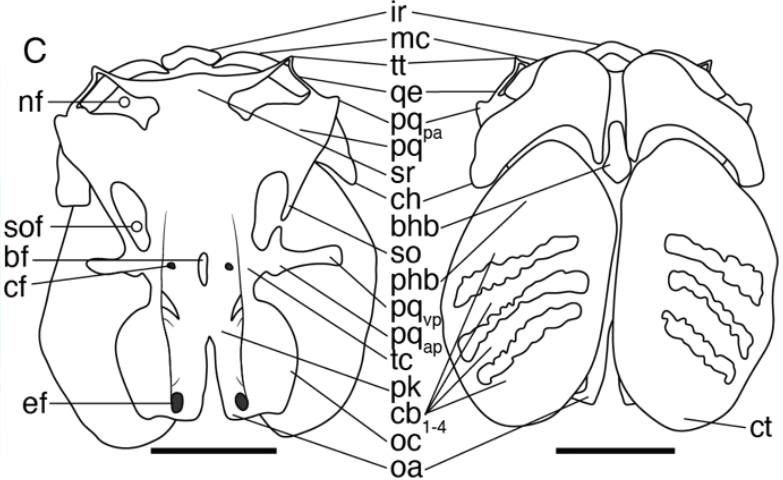

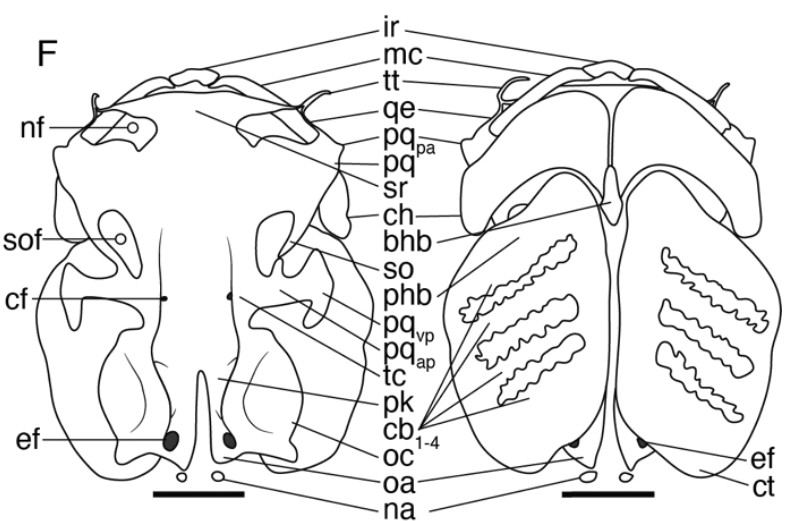

।

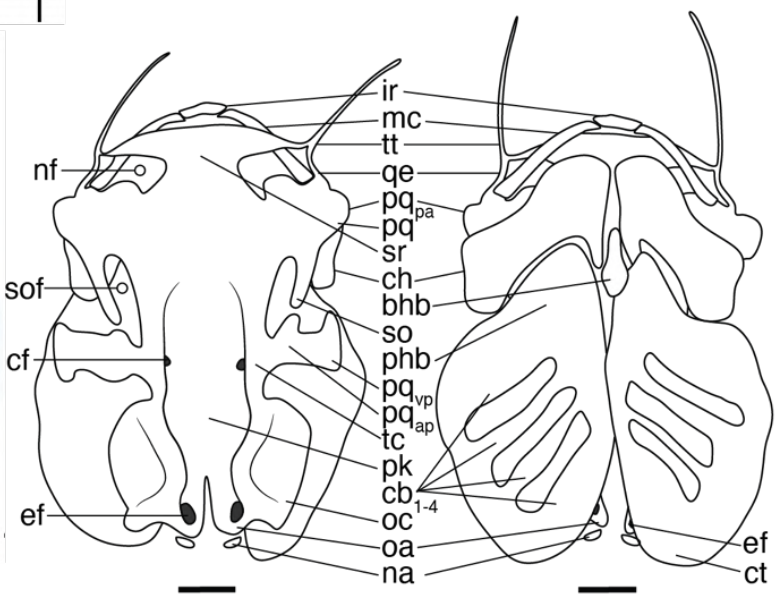

Figure 3.9. Development of the chondrocranium, stages 49 to 53 , in $X$. laevis. From left to right, each row consists of a photograph in dorsal (right) and ventral view (left), with an illustration in dorsal (right) and ventral (left) view. A-C, stage 49; D-F, stages 50-51; GI, stages 52-53. Lines to filled circles indicate small foramina and lines that end with open circles indicate larger foramina. Scale bars equal $1 \mathrm{~mm}$. Abbreviations: bf, basicranial fenestra; bhb, basihyobranchial; $\mathrm{cb}_{1-4}$, ceratobranchials one through four; cf, carotid foramen; ch, ceratohyal; ct, commisura terminalis; ef, exoccipital foramen; ir, infrarostral; mc, Meckel's cartilage; na, neural arches; nf, nasal foramen; oa, occipital arch; oc, otic capsule; phb, planum hyobranchiale; pk, parachordal cartilage; pq, palatoquadrate; $\mathrm{pq}_{\mathrm{ap}}$, ascending process of the palatoquadrate; $\mathrm{pq}_{\mathrm{pa}}$, pars articularis of the palatoquadrate; $\mathrm{pq}_{\mathrm{vp}}$, ventrolateral process of the palatoquadrate; qe, quadratoethmoidal; so, subocular cartilage; sof, subocular fenestra; sr, suprarostral plate; tc, trabecular cartilage; tt, tenticular cartilage. 
A

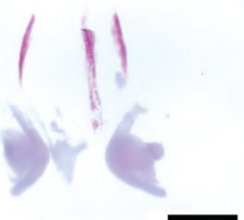

Stage 56

D

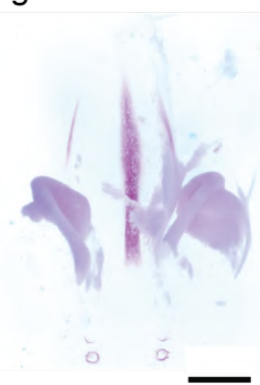

Stage 57

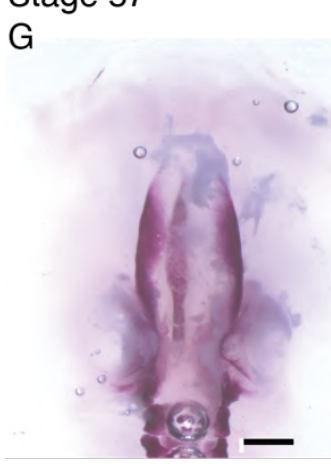

Stage 58-59

J

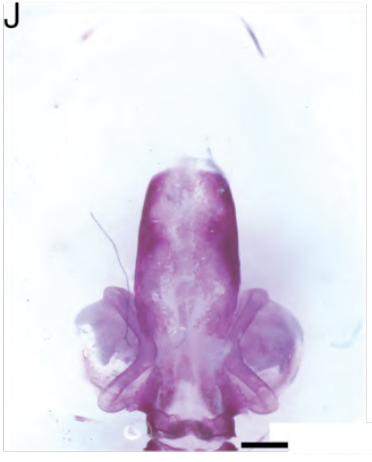

B

C
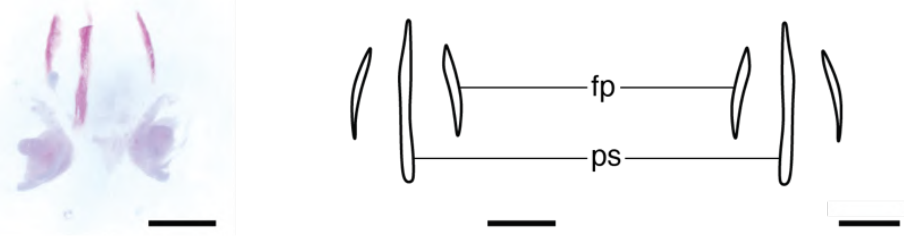

E

F
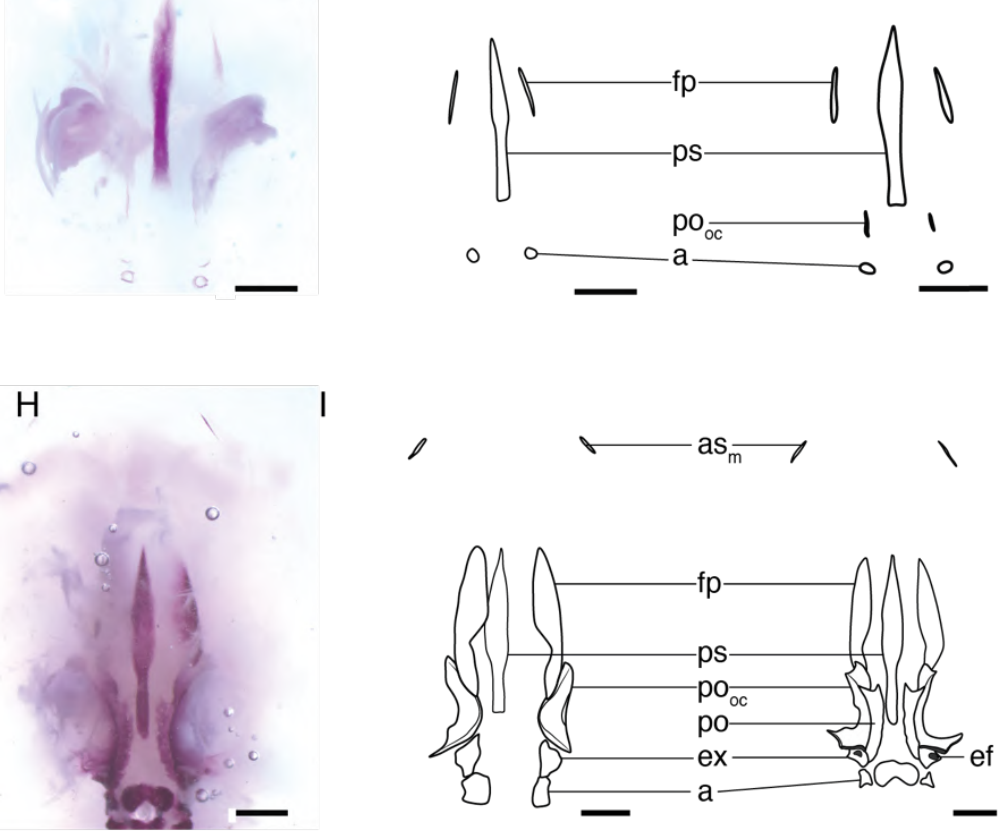

K
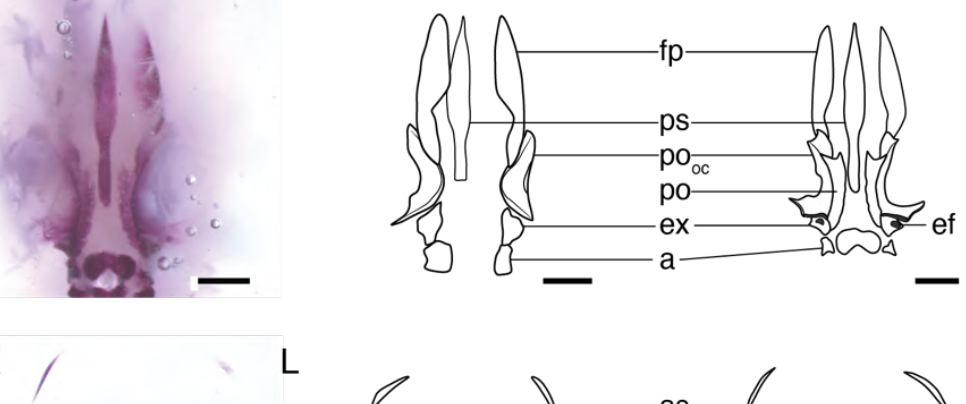

$\varnothing$
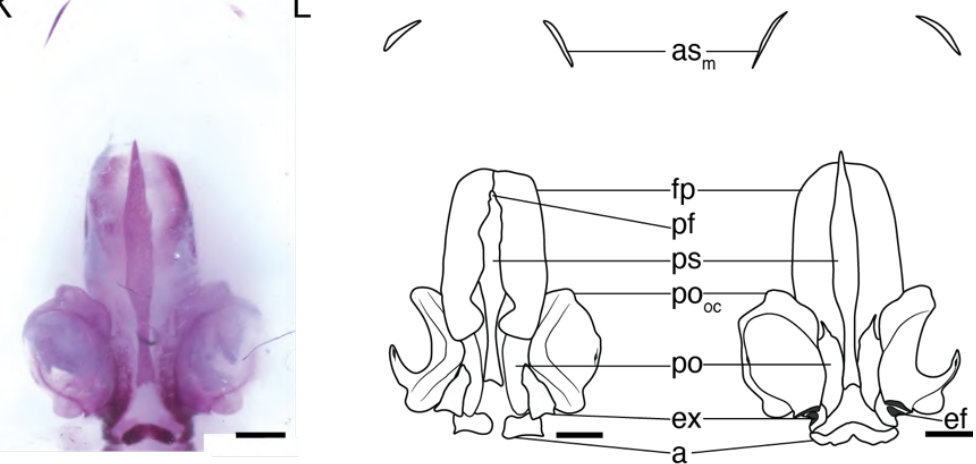

Figure 3.10. Stages 54 to $59, X$. laevis skull. From left to right, each row consists of a photograph in dorsal (right) and ventral view (left), with an illustration in dorsal (right) and ventral (left) view. A-C, stages 54-55; D-F, stage 56; G-I, stage 57; J-L, stages 58-59. Lines to filled circles indicate small foramina and lines that end with open circles indicate larger foramina. Scale bars equal $1 \mathrm{~mm}$. Abbreviations: a, atlas; $\mathrm{as}_{\mathrm{m}}$, medial angulosplenial; ef, exoccipital foramen; ex, exoccipital; fp, frontoparietal; po, prootic; po oc, otic capsule portion of the prootic; ps, parasphenoid. 

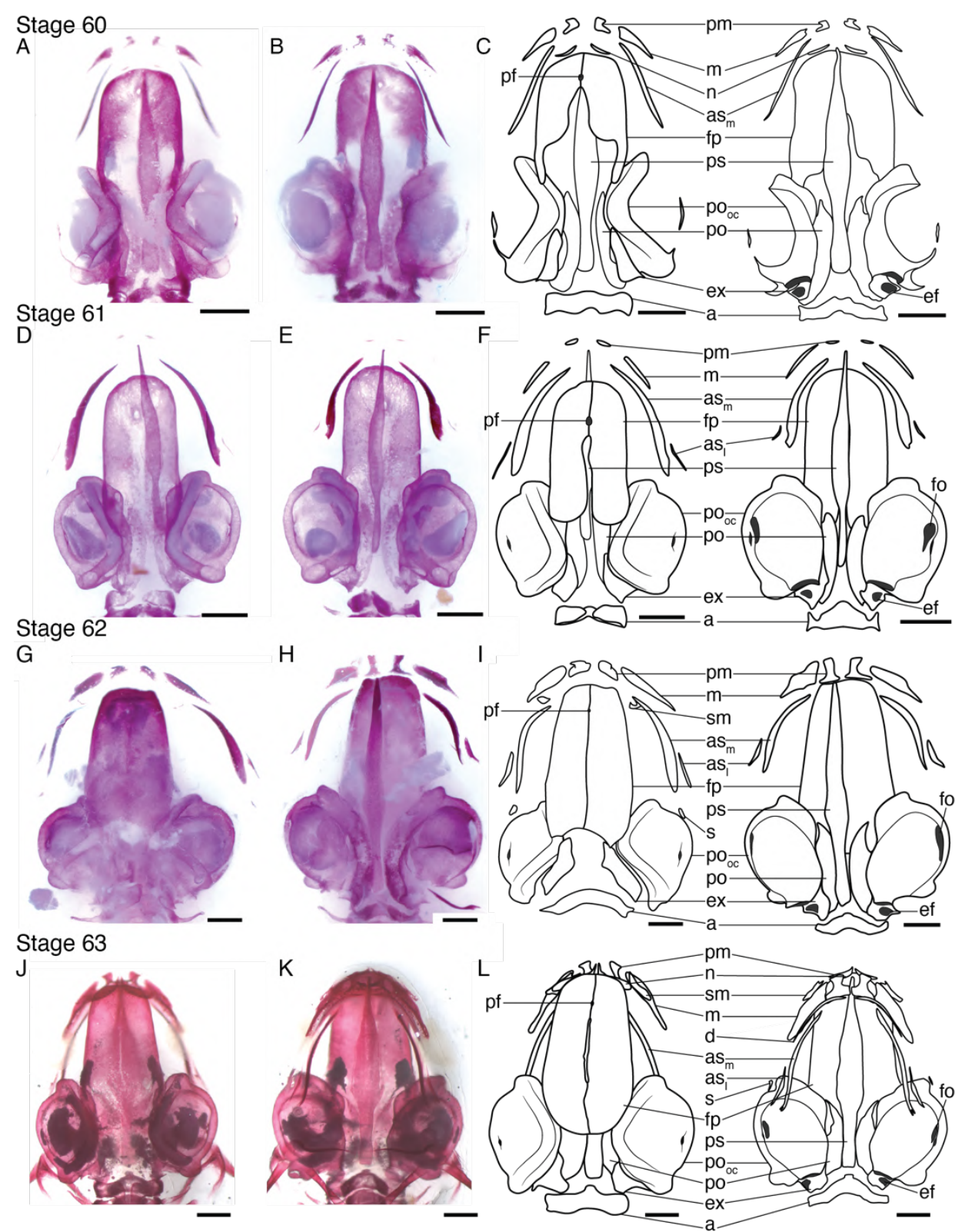

Figure 3.11. Stages 60 to $63, X$. laevis skull. From left to right, each row consists of a photograph in dorsal (right) and ventral view (left), with an illustration in dorsal (right) and ventral (left) view. A-C, stage 60; D-F, stage 61, upper and lower jaw elements were digitally rearticulated with the skull in panels $\mathrm{D}$ and $\mathrm{E}$, the composite photos were used as the basis of the line drawings in F; G-I, stage 62, upper and lower jaw elements were digitally rearticulated with the skull in panels $\mathrm{G}$ and $\mathrm{H}$, the composite photos were used as the basis of the line drawings in I; J-L, stage 63. Lines to filled circles indicate small foramina and lines that end with open circles indicate larger foramina. Scale bars equal 1 $\mathrm{mm}$. Abbreviations: a, atlas; as $\mathrm{s}_{\mathrm{m}}$, medial angulosplenial; as, lateral angulosplenial; $\mathrm{d}$, dentary; ef, exoccipital foramen; ex, exoccipital; fo, foramen ovalis; fp, frontoparietal; $\mathrm{m}$,

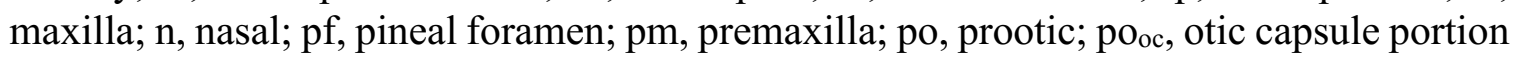
of the prootic; ps, parasphenoid; s, squamosal; sm, septomaxilla. 
Stage 64

A

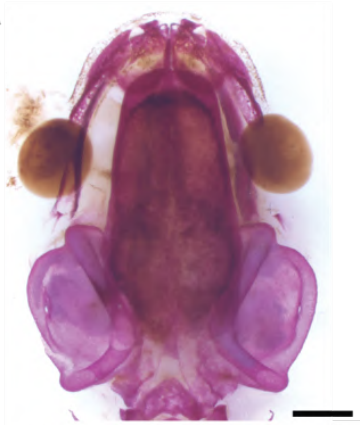

Stage 65

D

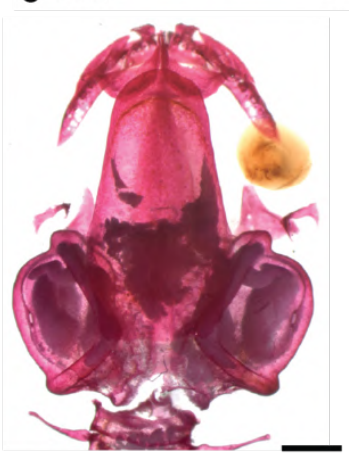

Stage 66

G

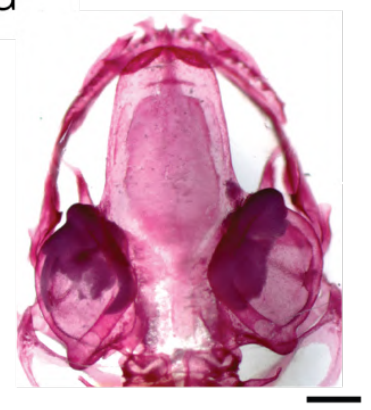

B

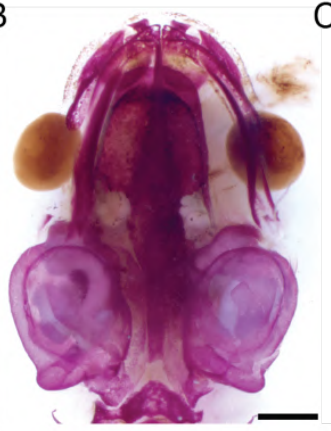

C

E

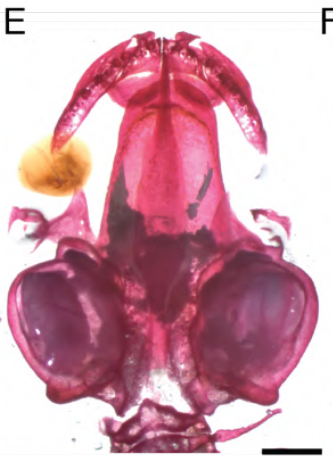

F
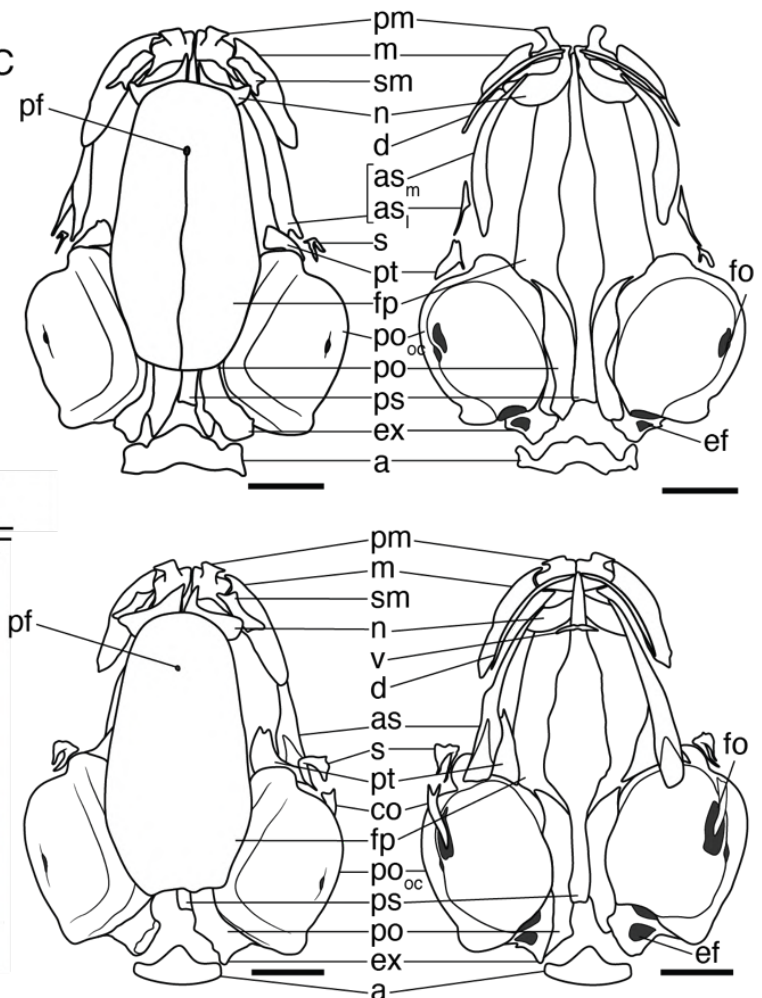

$\mathrm{H}$

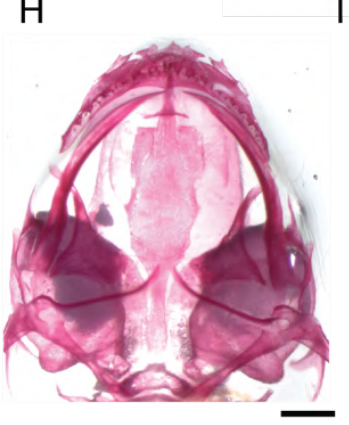

I

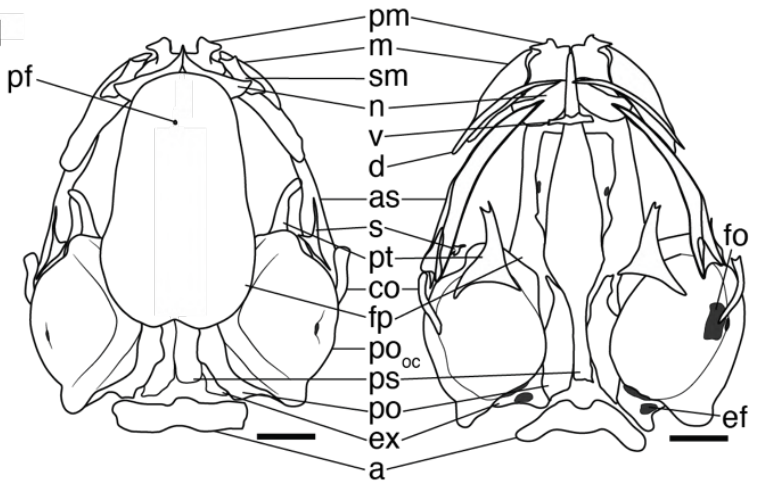

Figure 3.12. Stages 64-66, X. laevis skull. From left to right, each row consists of a photograph in dorsal (right) and ventral view (left), with an illustration in dorsal (right) and ventral (left) view. A-C, stage 64; D-F, stage 65; G-I, stage 66. Lines to filled circles indicate small foramina and lines that end with open circles indicate larger foramina. Scale bars equal $1 \mathrm{~mm}$. Abbreviations: a, atlas; as, angulosplenial with fused medial and lateral ossifications; as , medial angulosplenial; ası, lateral angulosplenial; co, columella; $d$, dentary; ef, exoccipital foramen; ex, exoccipital; fo, foramen ovalis; fp, frontoparietal; m, maxilla; $n$, nasal; pf, pineal foramen; pm, premaxilla; po, prootic; po oc, otic capsule portion of the prootic; ps, parasphenoid; pt, pterygoid; s, squamosal; sm, septomaxilla; v, vomer. 


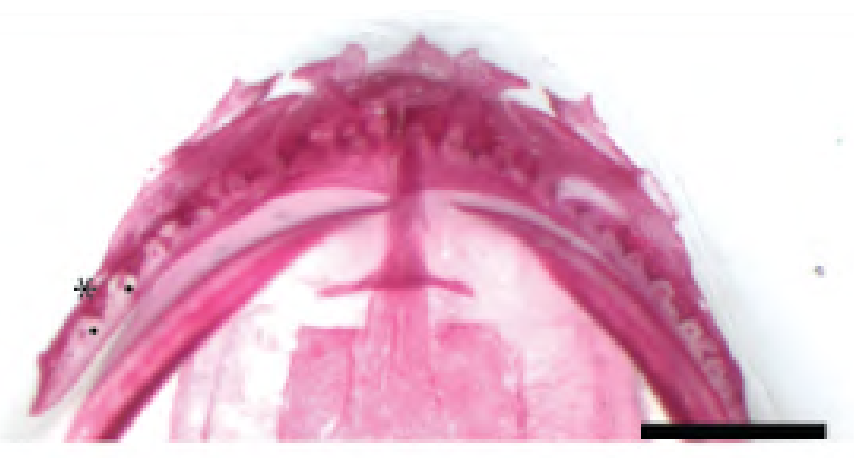

Figure 3.13. A stage $66 \mathrm{X}$. laevis specimen showing the two rows of teeth present in the maxilla and premaxilla. The larger, earlier developing tooth row (asterisk) is slightly anterior to the later developing, small tooth row (circles). Scale bar equals $1 \mathrm{~mm}$.

\subsection{4: Hypoglossal nerve morphology at the occiput}

\section{Ambystoma mexicanum}

The structures that the hypoglossal nerve innervates is well documented for salamanders (e.g., Platt, 1897; Norris, 1908; 1913; Wake and Lawson, 1973; Herrick, 1948; Roth et al., 1984); however, in A. mexicanum, where and how (i.e., inter- or intravertebrally) the hypoglossal nerve passes laterally through the axial skeleton has not yet been documented. Analysis of nerve-stained specimens of A. mexicanum show that in earlier developmental stages, before the interglenoid tubercle of the atlas (the first vertebral element) forms, the hypoglossal nerve extends laterally from the spinal cord just anterior to the atlas with three roots present (Figure 3.14A). In older specimens, where the interglenoid tubercle is ossified, the hypoglossal nerve extends laterally from the spinal cord via a foramen on the atlas (Figure 3.14B).

\section{Xenopus laevis}

The structures that the hypoglossal nerve innervates is well documented for frogs (e.g., Elliot, 1907; Barnard, 1940; Stuesse et al., 1983; Naumann and Olsson, 2018); however, where and how the hypoglossal nerve passes through the axial skeleton from the spinal cord has not yet been documented. Analysis of nerve stains in $X$. laevis specimens 
reveals that there is some variability in where the hypoglossal nerve passes laterally through the axial skeleton. In some specimens examined, the hypoglossal nerve passes laterally from the spinal cord with one root passing between the first and second vertebral elements and a second root passing between the second and third vertebral elements (Figure 3.14C). In other specimens, two roots of the hypoglossal nerve can be observed passing laterally from the spinal cord between the first and second vertebral elements (Figure $3.14 \mathrm{D})$.

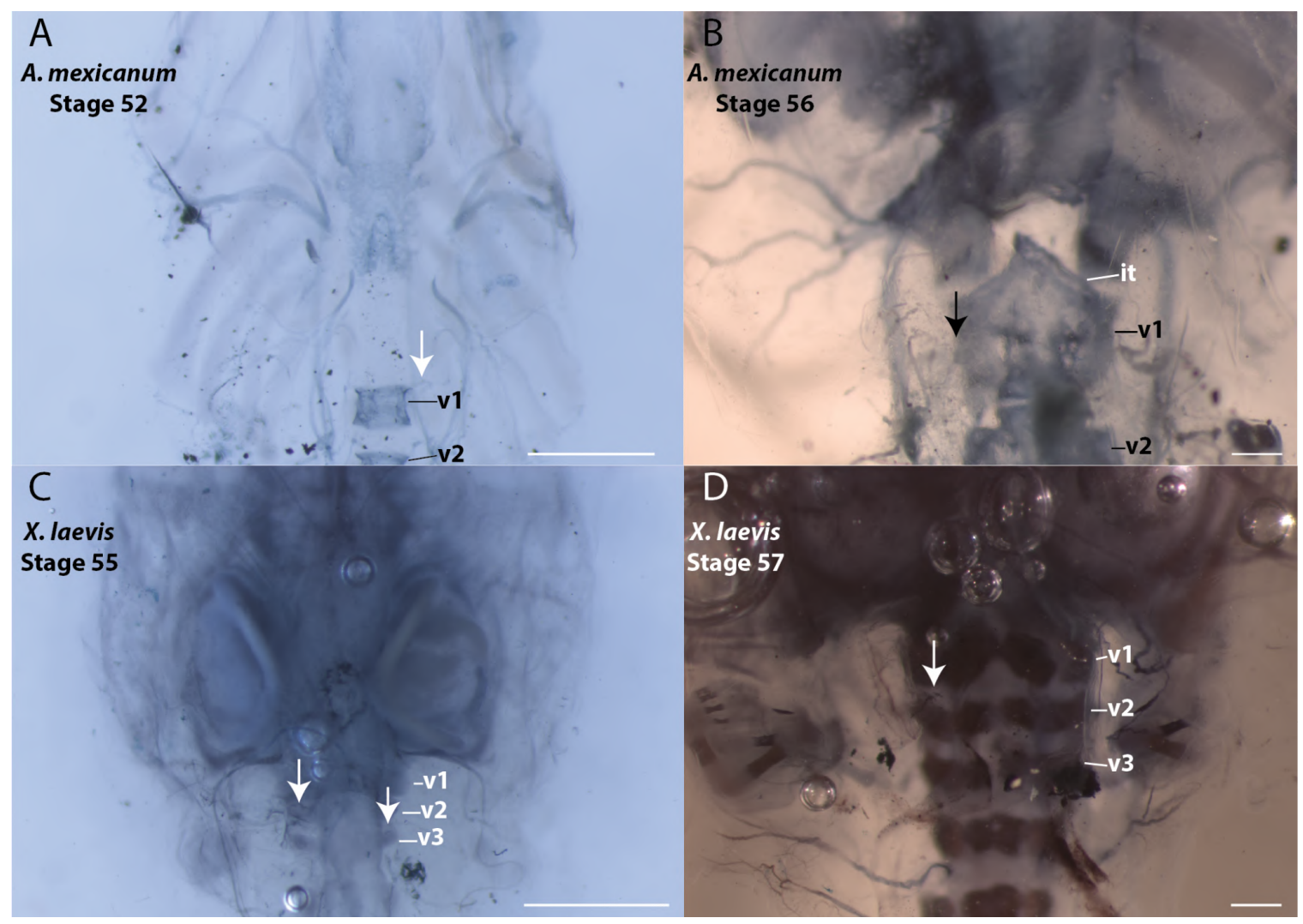

Figure 3.14. A. mexicanum and X. laevis specimens stained with Sudan Black to visualize nerves. A) in this stage $52 \mathrm{~A}$. mexicanum specimen, three hypoglossal nerve roots (arrow) are seen passing from the spinal cord through the axial skeleton anterior to the atlas (v1) as the interglenoid tubercle hasn't yet formed. B) in this stage 57 A. mexicanum specimen the hypoglossal nerve complex (arrow) is seen passing through a foramen on the anterior interglenoid tubercle (it) projection of the atlas. $\mathrm{C}$ ) in this stage $55 \mathrm{X}$. laevis specimen, the hypoglossal nerve complex (arrow) is seen passing between the first (v1) and second (v2) vertebral elements and the second and third (v3) vertebral elements. D) in this stage $57 X$. laevis specimen, the hypoglossal nerve complex (arrow) only passes between the first and second vertebral elements. Scale bars equal $1 \mathrm{~mm}$. 


\section{4: Discussion}

The goals of the present study were to 1) describe the somitic contributions to the post-otic (occipital) region of the chondrocranium in two lissamphibian model organisms, A. mexicanum for salamanders and $X$. laevis for frogs, 2) describe the long-term development of the skull, enhancing already published staging tables, and 3) describe the hypoglossal nerve morphology as it relates to the skull and vertebral elements.

\subsection{1: Somitic contributions to the occipital arch}

Fate mapping techniques have been applied to amniote model organisms such as the chicken and mouse (e.g., Couly et al., 1993; Burke et al., 1995; Huang et al., 2000) to study the nature of the somitic contribution to the occiput. For amphibians, previous analyses of somitic contributions to the head using modern cell lineage tracing techniques have only been performed on A. mexicanum (e.g., Piekarski and Olsson, 2007; Piekarski, 2009; Piekarski and Olsson, 2014). These studies focused on somitic contributions to the muscles, and less so on the somitic contributions to the skull. The present study aimed to fill this gap and confirmed the contribution of three somites to the occiput found by previous researchers (e.g., Piekarski and Olsson, 2007; Piekarski, 2009; Piekarski and Olsson, 2014). Piekarski (2009) noted somite one is very small during early somitogenesis and that it eventually merges with somite two. Here, a discrete somite one was not observed and, as such, was interpreted as already merged with somite two or already covered by migrating neural crest. Thus, somite one was not directly traceable; however, it is considered as contributing to the occiput via its close association with somite two. The entirety of somite two is found to contribute to the occiput (Figure 3.2D), and the anterior portion of somite three is found to contribute to the posteriormost portion of the occiput 
(Figure 3.2E). These data are in agreement with previous studies both for A. mexicanum (Piekarski, 2009), and for studies in other salamander species (e.g., Platt, 1898; Goodrich, 1911; Edgeworth, 1935; de Beer, 1937). Although some studies in salamanders report only two occipital somites, it is likely that these studies missed the small, transient first somite (summarized in de Beer, 1937 and Maddin et al., 2020). More accurately, two- and onehalf occipital somites are considered present in salamanders.

The present study represents the first use of modern cell-lineage tracing techniques to attempt to determine the somitic contributions to the occiput in X. laevis. Unfortunately, the label from these cell-lineage tracing techniques was only present in structures found within the jugular foramen in sections (Figure 3.3) and the label present in the whole-mount specimens was inconclusive (Figure 3.3A, B). However, the location of other structures within the developing embryo provides additional insights into how many somites contribute to the occiput in X. laevis. I noted during injection of X. laevis embryos that the anterior extent of the pronephros was consistently located at the border between somites two and three (Figure 3.1D). Previous research in A. mexicanum found that the pronephros was a consistent marker between somites that contribute to the occiput and axial somites, as it was always located between somites three and four (Figure 3.1B, Piekarski, 2009). In amniotes, the anterior extent of the pronephros is located posterior to the occipital somites, and its anterior extent is associated with somite eight in chick (Grinstein et al., 2013) and with somite seven in mice (Cartry et al., 2006). Using both the location of the pronephros, and the location of the hypoglossal nerve complex, I can at least confirm here that $X$. laevis likely does have fewer occipital somites than A. mexicanum, as the skull-neck boundary must be located at or anterior to the pronephros (at or within somite two). This 
interpretation is supported by the hypoglossal nerve complex extending laterally between the second and third vertebral elements in X. laevis, compared to a relatively more anterior position between the occiput and the first vertebral elements in A. mexicanum. Thus, if the somites retain their positional relationship with the hypoglossal nerve, as suggested by Maddin et al. (2020), only two somites remain in X. laevis to contribute to the occiput. While these cell-lineage data presented here may confirm the hypothesis first put forward by de Beer (1937) where the skull-neck boundary exists within somite three for salamanders and within somite two for frogs, in the future more analyses will need to be completed to confirm the somitic contribution to the occipital arch in X. laevis using GFPpositive somite grafting experiments.

In terms of somitic contributions to specific bones of the skull, somites two and three are confirmed here as contributing to the occipital arch, and thus the exoccipitals in particular, in A. mexicanum, while inconclusive evidence is presented that somite two contributes to the exoccipitals in X. laevis (Figure 3.2). In chick, where five occipital somites are confirmed, the first two somites contribute to the parasphenoid and to the supraoccipital, and all five occipital somites contribute to the exoccipitals and basioccipital (Couly et al., 1993; Huang et al., 2000). In A. mexicanum, somites two and three do not contribute to the parasphenoid. Although it is possible that the first, smaller somite contributes to the parasphenoid, this would need to be confirmed with cell-lineage studies. Unfortunately, the small, transient nature of the first somite in amphibians makes it difficult to study the contributions of this somite to the skull. Like in chick, multiple somites (somite 2 and part of somite 3) contribute to the exoccipital in A. mexicanum. The supraoccipital and basioccipital are absent in salamanders (see Chapter 2 for details). Again, given the 
fact that only exoccipitals are present in frogs, it is likely the two inferred occipital somites (somite one and the anterior portion of somite two) contribute to the exoccipitals in frogs.

These datasets represent the first concrete observations of somitic contributions to the skull for one amphibian model species, A. mexicanum, and represent the first evidence of possible somitic contributions to the skull in X. laevis. A clear understanding of the early phases of normal development of the skulls of these species are essential for future studies that attempt to manipulate and change somitic fate in order to determine the underlying developmental mechanisms for the origin of the lissamphibian condition. For example, in Chapter 4, the normal somitic contributions to the skull can now be compared to specimens where development is perturbed to see if somitic contributions can be experimentally changed.

\subsection{2: Ambystoma mexicanum and Xenopus laevis staging tables}

Complete descriptions of later development (i.e., chondrification and ossification) of the skull do not currently exist in the literature for A. mexicanum and are not currently easily integrated into the widely used staging tables for $X$. laevis. Below, some key findings from the staging table portions of this study are outlined.

\section{Ambystoma mexicanum}

The present study represents the first descriptive staging table for the skull of the model species A. mexicanum. The staging table of Nye et al. (2003) has served as a foundational tool for A. mexicanum researchers primarily working on limb regeneration. The complementary staging table of the skull provided here will further assist researchers studying many additional aspects of $A$. mexicanum development, as their full potential as a model system is realized. 
With this staging table of the skull in hand, some initial comparisons of skull development can be made between A. mexicanum and other Ambystoma species. The staging table presented here captures the majority of events in skull morphogenesis. However, the table of Nye et al. (2003) ends before late ossifying elements, such as the nasal, prootic, orbitosphenoid, and septomaxilla, appear. These elements do eventually ossify in A. mexicanum but they appear well after limb differentiation is complete, between 63 and 153 days post hatching (Rose, 2003; Smirnov and Vassilieva, 2005). A further extension of the Nye et al. (2003) would then be required to cover total skull development. For the purposes of the current work, however, the stages of Nye et al. (2003) were sufficient.

For the earlier ossifying skull elements, our results seem to generally corroborate previously established ossification sequences for A. mexicanum (Rose, 2003; Smirnov and Vassilieva, 2005). In comparison to other closely related species, the ossification sequence of the skull of the A. mexicanum progresses in a near identical sequence to that of close relatives A. maculatum, A. tigrinum, A. texanum, and A. talpoideum prior to metamorphosis (Bonebrake and Brandon, 1971; Reilly, 1987; Moore, 1991; Flick, 1992; Rose, 2003). This is an interesting result given the deviation in developmental trajectory, and further supports its use as a generalized model species in this regard.

One important distinction is the presence and ossification of a basioccipital. Rose (2003) lists an ossified basioccipital developing after the pterygoid and parasphenoid, but prior to the frontals. However, I did not find a basioccipital (cartilaginous nor ossified) in any of our specimens, nor do Smirnov and Vassilieva (2005) describe or figure a basioccipital for the same species. In close relatives of A. mexicanum, such as A. texanum 
and A. tigrinum, a basioccipital is not present either (Bonebrake and Brandon, 1971; Flick, 1992, Rose, 2003). Ambystoma mexicanum is the only species in Rose's (2003) included list of salamanders and their sequences of skull ossification where a basioccipital is listed as present. That the basioccipital is only listed as present in A. mexicanum by one source (Rose, 2003), and I did not observe it here, nor did other researchers observe it in other salamander species (see Rose, 2003 for a summary), it is likely that the basioccipital is indeed absent in A. mexicanum. These results make sense when considering the somitic contributions to the occiput. In amniotes, all five occipital somites contribute to the exoccipitals and the basioccipital (Couly et al., 1993; Huang et al., 2000). In lissamphibians, the exoccipitals are expanded compared to amniotes and their fossil relatives, but they do not have a basioccipital. Perhaps, at some point in their evolution, with fewer somites with which to make occipital elements, the somitic contributions to the skull expanded for the exoccipitals at the expense of a discrete basioccipital. Additionally, the formation of the occipital arch differs between amniotes and A. mexicanum. Here, I observed an occipital arch that in earlier developmental stages had an identical morphology to the axial neural arches (Figure 3.4). In chick, the occipital arch does not resemble the neural arches and is greatly expanded in size compared to A. mexicanum (Couly et al., 1993). The lack of an observed basioccipital in this study further supports the results in Chapter 2, where it was found that the basioccipital is absent in all lissamphibians and was likely lost at some point in the Amphibamiformes lineage (Figure 2.4).

\section{$\underline{\text { Xenopus laevis }}$}

The present study represents the first descriptive staging table for the skull of the model species $X$. laevis that describes and figures the presence and morphology of bones 
at every stage according to the staging table of Nieuwkoop and Faber (1994). Additionally, variation in the first appearance of bones is described for the first time for each bone. With this staging table in hand, researchers working on the skull can confidently know which stage a bone of interest is present, the temporal variation in the first appearance of each element, and their morphology at each stage corresponding directly to the stages in the already widely used Nieuwkoop and Faber (1994) staging table.

Here it was found that several key differences exist between the present study and previous studies of $X$. laevis skull development. In the present study, the parasphenoid was consistently the first element to ossify, whereas previous studies found that the frontoparietal was the first element to ossify. The first appearance of the maxilla and premaxilla is observed here to be both later and more variable than reported in previous studies. In the present study, the maxilla first appeared between stages 58 and 62, whereas the premaxilla first appeared between stages 60 and 62. In Trueb and Hanken (1992) both elements were instead consistently present by stage 59, whereas in Bernasconi (1951) they were reported as present by stage 55. Additionally, the septomaxilla appears as ossified first at stage 62 in the current study, but at stage 60 in other studies (e.g., Bernasconi, 1951; Trueb and Hanken, 1992). Finally, in the present study, I note that the collumella is present only by stage 65 while in previous studies it was present by stage 60 (Trueb and Hanken, 1992). Most of the other variation in the skull is variation by one or two stages, or minor differences in the ossification sequence.

The differences in timing and ossification sequences in the $X$. laevis skull as well as the variation in internal anatomy versus the easily staged external anatomy has been long noted in the literature (e.g., Trueb and Hanken, 1992; Lukas and Olsson, 2018). As in 
previous studies (Trueb and Hanken, 1992; Nieuwkoop and Faber, 1994), I found that the total lengths and overall size of Xenopus specimens are highly variable for specimens with the same external anatomy (therefore the same stage), especially in the stages when ossification is occurring (stage 54 and later). I found that the cartilaginous stages examined (stages 44 to 53 ) were less temporally variable than the stages where ossification was happening. Reasons for the observed temporal variation have been proposed to include environmental variations such as temperature, density, or the amount of light received by the tadpoles (Trueb and Hanken, 1992). Of note, the stages where I saw the most variation (stages 55 to 62) correspond to both an initial spike in the concentration of thyroid hormones found in X. laevis at the beginning of metamorphosis (stage 55) and to the peak of metamorphosis (stages 58-62) where thyroid hormones are also at their peak (Kawahara et al., 1991). The reason that the external anatomy fits into clearly defined stages, while the internal anatomy does not, could be a result of the condensed period of development that occurs during metamorphosis, and the internal anatomy is much more flexible than external anatomy during this time. After all, during metamorphosis, the head undergoes dramatic changes and restructuring from the juvenile chondrocranium to the adult frog skull (Slater et al., 2009).

As in A. mexicanum, a basioccipital was not found to be present in X. laevis. Unlike in A. mexicanum, however, the occipital arch was easily distinguished from the neural arches as it developed much earlier (stage 41) than the neural arches (present by stage 5051). The occipital arch was a small, narrow element during early development (Figure 3.8), smaller than the element present in A. mexicanum (Figure 3.4). It becomes attached to the parachordal cartilage early in development, by stage 43-44 (Figure 3.8), at which point it 
becomes difficult to differentiate from the nearby parachordal and prootic cartilages. Later in development, the exoccipital bones of $X$. laevis, manifested as the occipital condyles (Figure 3.12), are much larger than those observed in A. mexicanum (Figure 3.7). This is likely the result of the close association between the exoccipitals and the prootic, when compared to the seemingly floating exoccipitals in A. mexicanum. Likely, only a small portion of the occipital condyle comes from the somitic contributions to the skull and the rest of the element is formed by ossification(s) of the prootic. However, in order to determine the extent of the somitic contribution to the occipital condyles comes from the somites, cell-lineage tracing methods need to be developed and successfully carried out in X. laevis.

\subsection{3: Hypoglossal nerve complex}

The position of the hypoglossal nerve complex as it relates to the skull in amniotes is well known, while the association of the hypoglossal nerve complex as it relates to the axial skeleton in lissamphibians is less well-understood due to notable variation (e.g., Fürbringer, 1897; Platt, 1897; Elliot, 1907; Norris, 1908; Norris, 1913; de Beer 1937; Barnard, 1940; Wake and Lawson, 1973; Stuesse et al., 1983; Roth et al., 1984; Wake, 1992; Naumann and Olsson, 2018; Maddin et al., 2020). In salamanders, the hypoglossal nerve exits the spinal cord and passes laterally between the occiput the atlas (e.g., Platt, 1897; Wake and Lawson, 1973; Roth et al., 1984) or via a foramen on the atlas (e.g., Norris, 1908; Norris, 1913; Herrick, 1948). Prior to the formation of the interglenoid tubercle, an anterior projection of the atlas, the hypoglossal nerve passes anterior to the atlas in $A$. mexicanum (Figure 3.14A), as it does in plethodontids and Necturus (Platt, 1897; Wake and Lawson, 1973; Roth et al., 1984). Around stage 56, when the interglenoid tubercle 
forms, the hypoglossal nerve becomes enclosed by this element and passes laterally from the spinal cord via a foramen in the atlas (Figure 3.14B), as observed in A. tigrinum, Amphiuma, and Siren lacertina (Norris, 1908; Norris, 1913; Herrick, 1948; Edwards, 1976).

While the nerves of comparatively fewer frog species have been studied in detail, there may be more variation in the position of the hypoglossal nerve complex in frogs than in salamanders, although the position of the hypoglossal nerve complex in frogs is always located relatively more posteriorly than in salamanders. In Rana, the hypoglossal nerve complex passes laterally from the spinal cord between the first and second vertebral elements and previous researchers have noted that the first, second, and possibly third spinal nerves contribute to the hypoglossal nerve complex in X. laevis (e.g., Elliot, 1907; Barnard, 1940; Stuesse et al., 1983; Naumann and Olsson, 2018). Here, it was observed that there is variation in the portions of the axial skeleton associated with the hypoglossal nerve complex in $X$. laevis alone. In $X$. laevis, the hypoglossal nerve complex passes laterally between the first and second, and sometimes also the second and third vertebral elements (Figure 3.14C, D). Knowing these nerve landmarks in A. mexicanum and X. laevis are essential for future experiments that will attempt to manipulate the location of the skullneck boundary along the anterior-posterior axis.

The position of the hypoglossal nerve complex outside of the skull in lissamphibians also supports the data that fewer somites contribute to the occiput in lissamphibians. In chick, somite four gives rise to that part of the exoccipital that is traversed by the hypoglossal nerve (Huang et al., 2000). In A. mexicanum, somite four contributes to the posterior portion of the first vertebral element and the anterior portion of 
the second vertebral element. If the hypoglossal nerve continues to be associated with the fourth somite in lissamphibians, as in amniotes, then it makes sense that the hypoglossal nerve complex is associated with the first vertebral element, an element that somite four contributes to. Then, in $X$. laevis, if two somites contribute to the occipital arch as hypothesized, it would also make sense for the hypoglossal nerve complex to pass laterally between the first and second and the second and third vertebral elements. In X. laevis, the fourth somite would contribute to the second and third vertebral elements. Ultimately, a combination of cell-lineage tracing specifically for somite four and histological sections of embryos during somitogenesis will determine if the hypoglossal nerve complex remains associated with somite four in lissamphibians as in chick.

\section{5: Conclusions}

This study describes normal development of the skull in the amphibian model organisms $A$. mexicanum and $X$. laevis, from the somitic contributions to the occipital arch, to the development and morphology of skull cartilages and bones in all relevant stages within the two most widely used staging tables for these model species. These data are integrated with descriptions of the hypoglossal nerve as they relate to cartilaginous and ossified structures, so that I may better understand its potential to serve as a morphological landmark in the study of skull-neck boundary evolution as it has previously been proposed (Maddin et al., 2020). In addition, the developmental data presented here is easily integrated into previously published, widely used staging tables based primarily on external morphology for $X$. laevis (Nieuwkoop and Faber, 1994) and limb morphology for $A$. mexicanum (Nye et al., 2003), thereby expanding the utility of these tables into new areas of research (i.e., skull development). 
The reviewed literature reveals the number of somites that contribute to the occiput is variable among vertebrates. Previous researchers have speculated that the number of somites contributing to the skull increases first at the base of Tetrapoda and then again at the base of amniotes, making the amphibian morphology the basal condition for tetrapods (Augier, 1931), whereas others, using both fossil and extant morphological data, have suggested that instead the amphibian morphology is derived and the amniote morphology is the ancestral morphology for tetrapods (e.g., de Beer 1937; Romer and Edinger, 1942; Clack and Milner, 2009; Maddin et al., 2020). The present study, together with the study described in Chapter 2 based on fossil data, illustrates that indeed fewer somites contribute to the skull, and this likely resulted in a morphology where fewer bones contribute to the occiput, and a hypoglossal nerve complex that no longer passes through the skull via foramina. Fossil amphibians in the lineage leading to extant lissamphibians have these foramina and these bones (see Chapter 2 for details). Therefore, it appears that at some point during lissamphibian evolution, occipital somites became vertebral somites instead.

Given what we know about the patterning of somites, we can look to changes in Hox gene expression during development along the anterior-posterior axis (see Chapter 4 for details). The normal developmental data presented here can now be used as a baseline to test the hypothesis that changing Hox gene expression domains may have driven changes in the location of the skull-neck boundary by examining gene expression along the anterior-posterior axis and comparing it to experimental specimens where Hox gene expression domains have been manipulated. 


\section{Chapter 4: Weird bois, the experimental effects of perturbed Hox genes on the development of the skull-neck boundary}

\section{1: Introduction}

The posterior (post-otic) portion of the skull is derived from the anteriormost somites of the embryonic anterior-posterior axis, where the posteriormost somite that contributes to the skull contains the posterior limit of the skull. Early research into embryonic development describes highly variable numbers of somites within the occiput of tetrapods, where amniotes, in general, are thought to possess more than living nonamniotes (lissamphibians) (see de Beer, 1937 and Maddin et al., 2020 for summaries of somite contribution to the occiput across several vertebrate lineages). One currently proposed hypothesis of the evolution of the skull is that the amniote condition is derived relative to non-amniotes and that non-amniotes represent the ancestral tetrapod condition; thus, at some point during their evolution amniotes recruited additional somites into the occipital region of the skull (e.g., Augier, 1931; Couly et al., 1993). However, a recent review taking into consideration osteological correlates of somitic contribution, in order to include fossil taxa (Maddin et al. 2020), resurrected a second hypothesis that amphibians are instead secondarily derived in their reduced occipital morphology and the amniote condition is the actual ancestral tetrapod condition (de Beer 1937; Romer and Parsons 1977).

The previous two chapters have shown that, when including data from the fossil record, the condition of the occiput seen in living amphibians is derived and the ancestral state for tetrapods is to incorporate more somites into the skull, as in amniotes (Chapter 2). The normal development of the skull, specifically the occiput, and the resulting phenotypes 
were examined in two lissamphibian model organisms (Chapter 3), and it was shown that lissamphibians have lost bones in the occiput that are present in their ancestors (Chapter 2, Chapter 3) and additionally, the key morphological marker - a portion of the hypoglossal nerve complex - is now positioned outside of the skull likely as a result of reduced numbers of occipital somites (Chapter 3). With these data in hand, the next step is to determine the underlying developmental mechanisms that may have resulted in the acquisition of the unique lissamphibian morphology.

Changes in Hox gene expression domains are excellent candidates for playing a role in changing morphologies, and thus fate, of somite-derived structures along the anterior-posterior axis. Hox genes are part of a group of genes called homeobox genes that share similar homeobox sequences of approximately 180 base pairs in length (McGinnis and Krumlauf, 1992). Homeobox genes, including Hox genes, are important for patterning an embryo along the anterior-posterior axis (Gaunt et al., 1988; McGinnis and Krumlauf, 1992). The pattern of expression for Hox genes and the combination of which Hox genes are expressed where along the anterior-posterior axis, gives each somite its identity (i.e., cervical, thoracic, etc.), and the anterior limit of certain paralogous groups mark transitions between these regions along the anterior-posterior axis (e.g., Figure 1.5; Kessel and Gruss, 1991; Ramírez-Solis et al., 1993; Burke et al., 1995; Nowicki and Burke, 2000; Wellik and Capecchi, 2003).

Hox genes have several fascinating traits. They exhibit colinear expression, where they are expressed along the anterior-posterior axis in the same order they appear on their chromosome (McGinnis and Krumlauf, 1992; Krumlauf, 1994). Colinear expression also means that Hox genes that are from the same paralogous group (e.g., hoxa3, hoxb3, and 
hoxd3) have similar expression patterns and that more Hox genes end up being expressed in the posterior region of the embryo than in the anterior region due to the nested pattern of their colinear expression (Manzanares et al., 2001; Nolte et al., 2019). While some studies have shown that Hox genes also exhibit temporal collinearity, i.e., anterior Hox genes are expressed earlier in development than posterior Hox genes (Monteiro and Ferrier, 2006; Durston and Zhu, 2015; Zhu et al., 2017), temporal collinearity may not be a ubiquitous phenomenon. For example, in Xenopus species, during early development many Hox genes are expressed simultaneously, or posterior genes may be expressed earlier in development than anterior Hox genes (Kondo et al., 2017; Kondo et al., 2019). Hox genes in a paralogous group can also compensate for one another when one member is knocked down (Burke et al., 1995; Manzanares et al., 2001; Mallo et al., 2010) and the property of posterior dominance can result in posterior Hox genes compensating for loss of expression of more anterior Hox genes (Hooiveld et al., 1999; Mallo et al., 2010; Gummalla et al., 2014). As such, loss of function experiments of single, anterior Hox genes tend not to affect the morphology of the axis at the posterior end of the animal.

Hox gene expression domains can be perturbed by certain chemicals. For example, many experiments aimed at understanding the function of Hox genes were conducted using exogenous retinoic acid - a chemical known to perturb Hox gene expression domains (e.g., Maden, 1983; Durston et al., 1989; Kessel et al., 1990; Sive et al., 1990; Ruiz i Altaba and Jessel, 1991). Retinoic acid, which is an active form of vitamin A, plays a role in patterning many embryonic tissues (Ruiz i Altaba and Jessel, 1991; Langston and Gudas, 1992; Wobus et al., 1997; Maden, 2000; Durston, 2019). For the present study, I am interested in how retinoic acid can affect Hox gene expression patterns and describing the impacts of 
perturbed Hox gene expression on patterning the somites, specifically the anteriormost somites involved in skull formation.

Retinoic acid is present during somitogenesis and helps to regulate the formation of somites. During somitogenesis, somites form at regular intervals from the presomitic paraxial mesoderm (Figure 1.1). This rhythmic formation of somites is, at present, best described by the Clock and Wavefront model, where oscillatory expression of genes occurs in the presomitic mesoderm at regular intervals. (Figure 4.1; Cooke and Zeeman, 1976; Gomez and Pourquié, 2009; Mallo, 2015). The clock portion refers to the cyclic gene expression that occurs within the presomitic mesoderm and the regular intervals at which somites form. The wavefront portion refers to the progression of somitogenesis in an anterior-posterior direction as a result of a molecular oscillation mechanism that passes over the presomitic mesoderm at the regular intervals produced by the clock portion of the model (Cooke, 1975; Cooke and Zeeman, 1976; Pourquié, 2003; Gomez and Pourquié, 2009). The wavefront is controlled by opposing gradients of retinoic acid, which has higher expression in the anterior region, and FGF/Wnt signalling pathways, which has higher expression in the posterior region (Figure 4.1; Gomez and Pourquié, 2009). The end result of somitogenesis is a species-dependent number of somites occurring on either side of the neural tube, starting immediately posterior to the otic vesicles (Dequéant and Pourquié, 2008). 


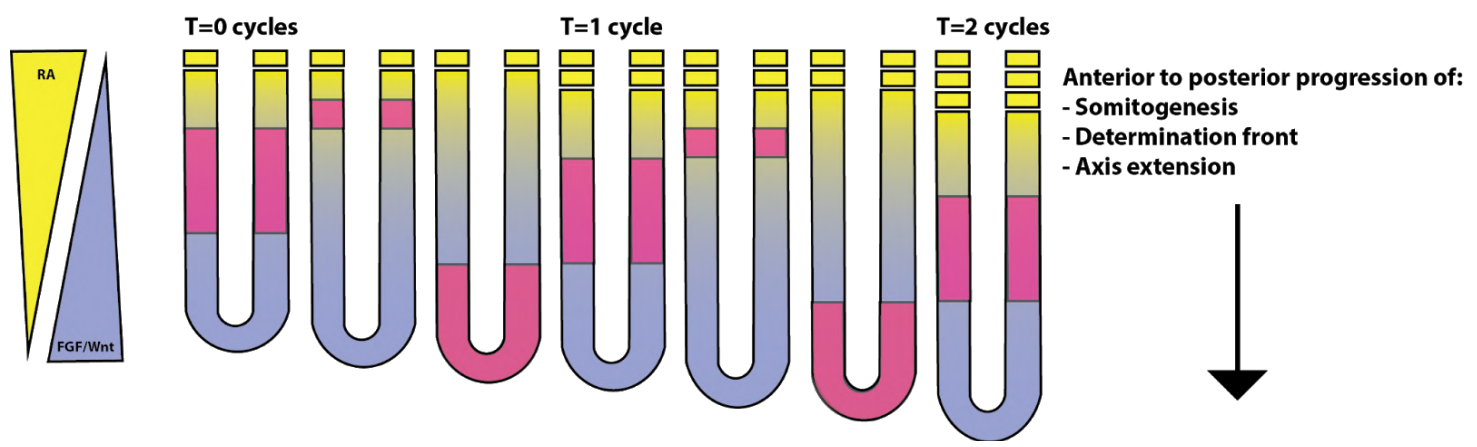

Figure 4.1. The Clock and Wavefront model showing the temporal sequence of somite formation in the paraxial mesoderm. Opposing gradients of retinoic acid (yellow) and FGF and Wnt (purple) are expressed along the anterior-posterior axis. The clock is the result of cyclic waves of gene transcription (pink) that occur at regular intervals. As the wavefront passes through the anterior region, a somite forms. Modified from Dequéant and Pourquié (2008).

In addition to its role in somitogenesis, retinoic acid also plays a role in switching on Hox genes. Retinoids, like retinoic acid, enter the nucleus and bind to retinoic acid receptors, which are members of the nuclear hormone class of receptors (Swindell and Eichele, 1999; Zhu et al., 2017; Ghyselinck and Duester, 2019; Nolte et al., 2019). Retinoic acid receptors then form heterodimeric complexes that bind to retinoic acid response elements (Figure 4.2; Swindell and Eichele, 1999; Nolte et al., 2019). Many genes that are expressed during early development, including Hox genes, contain upstream retinoic acid response elements (Figure 4.2). Hox genes are therefore activated in developing embryos by retinoic acid (Durston et al., 1989; Boncinelli et al., 1991; Ruiz i Altaba and Jessel, 1991; Marshall et al., 1994; Kolm and Sive, 1995). Hox genes contain enhancers that mediate their response to retinoic acid and regulate which Hox genes are expressed where and changing the concentration of retinoic acid in the embryo can not only change which Hox genes are expressed within a segment but also the location of their expression across the somites (Langston and Gudas, 1992; Blumberg et al., 1997). Application of exogenous retinoic acid causes the posteriorization of somites, while inhibiting retinoic acid or 
mutations that cause certain Hox genes not to be expressed causes the anteriorization of segments (e.g., Figure 1.6; Durston et al., 1989; Kessel et al., 1990; Ruiz i Altaba and Jessel, 1991; Conlan and Rossant, 1992; Ramírez-Solis et al., 1993; Manley and Capecchi, 1997; McNulty et al., 2005). When retinoic acid response elements are mutated so they cannot interact with retinoic acid, Hox gene expression moves posteriorly (Kobrossy et al., 2006). As a result of their nested expression and the location of endogenous retinoic acid within the developing embryo, Hox genes that are located closer to the 3 ' end of the chromosome and that have a more anterior expression limit are expressed earlier during development and are more sensitive to retinoic acid concentration than Hox genes located closer to the 5' end (Krumlauf, 1994; Blumberg et al., 1997; Nolte et al., 2019).
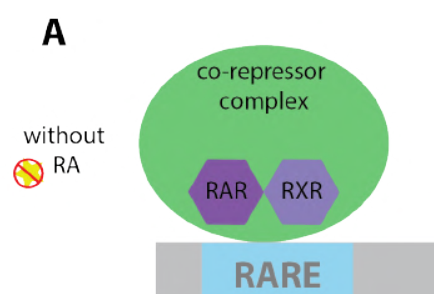

RARE
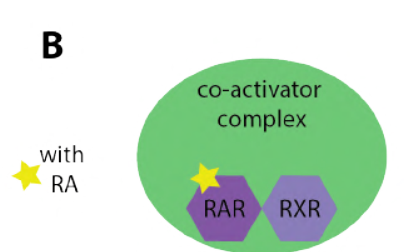

\section{RARE}

Histone deacetylation and repression of transcription

Hox1 Hox2 Hox3 Hox4

\section{Histone acetylation and activation of transcription}

Figure 4.2. How retinoic acid interacts with Hox genes. A) Without retinoic acid binding to retinoic acid receptors (RARs), transcription is repressed. B) In the presence of retinoic acid binding to retinoic acid receptors, which in turn interact with retinoic acid response elements (RAREs), transcription is activated. Modified from Marlétaz et al. (2006).

Numerous experiments have shown that changing the expression domains of Hox genes either via applying exogenous or inhibiting retinoic acid, or by knocking down specific Hox genes, produces segmental or homeotic transformations along the anteriorposterior axis of elements derived from paraxial mesoderm (Kessel et al., 1990; Conlan 
and Rossant, 1992; Ramírez-Solis et al., 1993). In transgenic mice, deregulating the expression of hoxa7 causes malformations in the occipital arch including additional ossification on the exoccipital compared to controls, and fusion between the basioccipital and the vertebral column (Kessel et al., 1990). Moving the anterior limit of hoxa7 expression anteriorly and inhibiting hoxb4 and hoxd4 expression also resulted in transformed cervical vertebral elements (Kessel et al., 1990; Ramírez-Solis et al., 1993; Horan et al., 1995). By moving hoxa 7 expression anteriorly, the morphology of the second vertebral element (the axis) resembled the third vertebral element and an additional vertebral element between the skull and the first vertebral element (the atlas) developed, which the authors termed a proatlas (Kessel et al., 1990). By moving the anterior limit of the expression domains of hoxb4 and hoxd4 posteriorly, or by simply inhibiting hoxb4 and hoxd4 expression, the morphology of the second vertebral element resembled that of the first vertebral element (Ramírez-Solis et al., 1993; Horan et al., 1995).

While the literature is dominated by studies on amniote models, some similar experiments using exogenous retinoic acid have been conducted in amphibians. For example, applying exogenous retinoic acid to Xenopus laevis embryos at developmental stages prior to or during mid-neurulation stages causes microcephaly that results in the loss of anterior structures such as the eyes, nasal pits, forebrain, and midbrain, and the cement gland (Durston et al., 1989; Sive et al., 1990; Ruiz i Altaba and Jessel, 1991). Retinoic acid additionally causes truncated tail development (Ruiz i Altaba and Jessel, 1991), which also occurs in loss of function experiments for hoxc6 (e.g., Zhu et al., 2017a). Applying retinoic acid to $X$. laevis embryos at later developmental stages does not cause such drastic effects to anterior structures but does still affect posterior structures such as tail development 
(Durston et al., 1989; Sive et al., 1990; Ruiz i Altaba and Jessel, 1991). Previous gene expression pattern research in amniotes and in X. laevis has shown that anteriorly expressed Hox genes can have their anterior boundary shifted with the application of exogenous retinoic acid (e.g., Durston et al., 1989; Kessel et al., 1990; Ruiz i Altaba and Jessel, 1991; Conlan and Rossant, 1992; Ramírez-Solis et al., 1993; Manley and Capecchi, 1997; McNulty et al., 2005). However, none of these studies looked at the consequences of changing gene expression patterns on late developing structures, like the skull.

Inhibiting retinoic acid in $X$. laevis also causes morphological abnormalities (e.g., Schuh et al., 1993), as in amniote model organisms. Inhibition can be induced using citral, a chemical that inhibits the oxidation of retinol to retinoic acid, and thus results in lower concentrations of endogenous retinoic acid within the developing embryo (Schuh et al., 1993; Tanaka et al., 1996). Specifically, the developing branchial arches bulge excessively, and the heads are larger and expanded laterally ventral to the eyes in comparison to controls (Schuh et al., 1993). As in exogenous retinoic acid studies, these researchers did not examine the consequences of applying citral on the resulting morphology of the skull.

In Ambystoma mexicanum some experiments studying Hox gene expression patterns and other experiments on exogenous retinoic acid have been conducted mostly in studies of limb regeneration (e.g., Maden, 1983; Gardiner et al., 1995; Torok et al., 1998; Carlson et al., 2001; Bickelmann et al., 2018) and lateral line development (e.g., Gibbs and Northcutt, 2004). For example, it has been found that applying retinoic acid to a regenerating limb causes serial repetitions along the proximal to distal axis, resulting in regenerated limbs with morphologies that range from extra carpels to entirely duplicated limbs (Maden, 1983). In the regenerating limbs, applying retinoic acid also decreases the 
concentration of hoxal3 (Gardiner et al., 1995). In the lateral lines, high concentrations of retinoic acid caused a reduction in the number of neuromasts present (Gibbs and Northcutt, 2004). The treatment additionally caused microcephaly, eye and otic capsule abnormalities, as seen in X. laevis and amniotes (Gibbs and Northcutt, 2004). As in $X$. laevis, these studies did not examine consequences on the skull.

The goal of the present study is to test the hypothesis that perturbing Hox gene expression domains can cause homeotic transformations at the occiput in A. mexicanum and X. laevis. To further understand the potential evolutionary mechanism that gave rise to the derived lissamphibian condition, manipulation of Hox gene expression domains were performed using amphibian model organisms A. mexicanum and X. laevis. Exogenous retinoic acid was administered to embryos during early somitogenesis via their water to move Hox gene expression anteriorly, and citral, which inhibits retinoic acid expression (Schuh et al., 1993; Kronmiller et al., 1995; Di Renzo et al., 2007), was administered also via water to move Hox gene expression posteriorly. The resulting embryos were allowed to grow to various post-hatching developmental stages in order to examine the resulting skull morphologies from these treatments. In addition to describing these resulting morphologies, I used somite lineage tracing experiments in A. mexicanum and nerve staining analysis in both A. mexicanum and X. laevis to confirm the occurrence of induced homeotic transformations. The results are discussed as they relate to the evolution of the skull-neck boundary in tetrapods. 


\section{2: Methods}

\subsection{1: Animal care and breeding}

Ambystoma mexicanum

Adult A. mexicanum breeding pairs are maintained as part of the breeding colony in the Maddin Lab at Carleton University, Ottawa, Canada (original source: Ambystoma Stock Center, Kentucky, IL). All A. mexicanum are housed and cared for in accordance with the Canadian Council on Animal Care and Carleton University's Animal Care Committee approved animal use protocol (AUP \#102951). Clutches of A. mexicanum embryos were obtained from natural breeding events between male and female leucistic mature adults. Embryos were dejellied (i.e., the protective membranes surrounding the embryo were removed) manually using forceps after neural tube closure and kept in agarlined Petri dishes filled with autoclaved $40 \%$ Holtfreter's solution in an incubator at $18^{\circ} \mathrm{C}$. At approximately stage 45 (Bordzilovskaya et al., 1989) larvae were transferred to containers containing $20 \%$ Holtfreter's solution and maintained at $18^{\circ} \mathrm{C}$ on a 12 -hour day and 12-hour night light cycle. Once able to feed, hatched larvae were fed a diet of larval brine shrimp once daily. Embryos were staged according to Bordzilovskaya et al. (1989) and larvae were staged according to Nye et al. (2003).

\section{Xenopus laevis}

Adult $X$. laevis breeding pairs are maintained as part of the breeding colony in the Maddin Lab at Carleton University, Ottawa Canada (source: Marine Biology Laboratories, Massachusetts). All X. laevis are housed and cared for in accordance with the Canadian Council on Animal Care approved animal and Carleton University's Animal Care Committee approved animal use protocol (AUP \#102952). In order to induce spawning, 
female $X$. laevis were initially injected with $50 \mathrm{IU}$ (international units) of human chorionic gonadotropin (HCG; Sigma C1063). After 48 hours, the female was injected again with $500 \mathrm{IU}$ of HCG. At the time of the female's second injection, a male was injected with 50 IU of HCG. Both frogs were then placed together in a nuptial tank with artificial plants and tubing. The next morning, embryos were collected and chemically dejellied using a $2 \%$ solution of buffered L-Cysteine free base (Fisher Scientific BP376100). X. laevis embryos were stored in agar lined Petri dishes filled with autoclaved 10\% Holtfreter's solution. At approximately stage 46 (Nieuwkoop and Faber, 1994), tadpoles were transferred to small tanks filled with $20 \%$ Holtfreter's solution. Once able to feed, tadpoles were fed a diet of Tropical Pro Defence Hi-Protein food for fry once daily. Embryos and tadpoles were staged according to Nieuwkoop and Faber (1994).

\subsection{2: Retinoic acid experiments}

\section{Ambystoma mexicanum}

Stage 28 to 35 (Bordzilovskaya et al., 1989) embryos were placed in a $40 \%$ Holtfreter's solution containing final concentrations of $0.01 \mu \mathrm{M}$ or $0.05 \mu \mathrm{M}$ retinoic acid (Sigma Aldrich R2625) dissolved in dimethyl sulfoxide solution (DMSO; Fisher 160239). Previous research in X. laevis has shown that lower concentrations of retinoic acid do not produce head defects (Durston et al., 1989), as A. mexicanum embryos are larger than $X$. laevis embryos, both the minimum dose required to produce defects in X. laevis and stronger concentrations were attempted here. These specimens were left in their treatment for four or seven days and then raised under normal conditions until hatching stage 46 or later (Bordzilovskaya et al., 1989). Control specimens were placed in $40 \%$ Holtfreter's solution and an equal volume of DSMO alone as that added to experimental animal water. 
Once specimens reached the desired stage (i.e., when at least all elements of the chondrocranium were present as determined in Chapter 3), they were anesthetized by adding a few drops of 4\% MS-222 (ethyl 3-aminobenzoate methanesulfonate [SigmaAldrich E10521]) to their container. Once vital signs disappeared the specimens were then fixed in 10\% neutral buffered formalin (NBF; Fisher SF98) overnight at room temperature and then dehydrated for storage in a $70 \%$ ethanol solution at room temperature. Total specimen numbers for each treatment can be found in Appendix G.

\section{Xenopus laevis}

Embryos at developmental stages ten or eleven (Nieuwkoop and Faber, 1994) were placed in a $10 \%$ Holtfreter's solution containing final concentrations of $0.01 \mu \mathrm{M}$ or 0.001 $\mu \mathrm{M}$ retinoic acid dissolved in DMSO solution for 30- and 60-minute pulses, or overnight for seventeen hours. Previous research has shown that lower concentrations of retinoic acid, and embryos first exposed to exogenous retinoic acid after stage fourteen, do not produce head defects in X. laevis (Durston et al., 1989). Control specimens were placed in $10 \%$ Holtfreter's solution and an equal volume of DSMO alone as that added to experimental animal water. Specimens were then raised until post-hatching stages of development (Nieuwkoop and Faber, 1994) under normal conditions. Once specimens reached the desired stage (i.e., when at least all elements of the chondrocranium were present as determined in Chapter 3), they were anesthetized by adding a few drops of $4 \%$ MS-222 to their container. Once vital signs disappeared, specimens were then fixed in 10\% NBF overnight at room temperature and then dehydrated for storage in a $70 \%$ ethanol solution at room temperature. Total specimen numbers for each treatment can be found in Appendix H. 


\subsection{3: Citral experiments}

Ambystoma mexicanum

Stage 25 to 30 (Bordzilovskaya et al., 1989) embryos were placed in a $40 \%$ Holtfreter's solution containing final concentrations of $5 \mu \mathrm{M}, 10 \mu \mathrm{M}$, or $20 \mu \mathrm{M}$ citral (Acros Organics AC110441000) dissolved in DMSO solution. Stronger doses of citral were used in an earlier experiment on limb regeneration (Scadding, 1999), however, the embryos did not tolerate citral treatment well. The doses used here were the maximum tolerated by embryos, where at least one embryo survived treatment. Specimens remained in citral treatments for a total of two weeks and were then raised under normal conditions to post-hatching stages of development (Nye et al., 2003). Control specimens were placed in $40 \%$ Holtfreter's solution and an equal volume of DSMO alone as that added to experimental animal water. Once specimens reached the desired stage (i.e., when all the cartilaginous elements of the skull are present as determined in Chapter 3), they were anesthetized by adding a few drops of $4 \%$ MS-222. The specimens were then fixed in $10 \%$ NBF overnight at room temperature and then dehydrated for storage in a $70 \%$ ethanol solution at room temperature. Total specimen numbers for each treatment can be found in Appendix G.

$\underline{\text { Xenopus laevis }}$

Stage 10 and 11 (Nieuwkoop and Faber, 1994) embryos were placed in a 10\% Holtfreter's solution containing final concentrations of $1 \mu \mathrm{M}, 2.5 \mu \mathrm{M}, 5 \mu \mathrm{M} 10 \mu \mathrm{M}, 15$ $\mu \mathrm{M}$, or $20 \mu \mathrm{M}$ citral dissolved in dimethyl sulfoxide solution for 24 hours. Control specimens were placed in 10\% Holtfreter's solution and an equal volume of DSMO alone as that added to experimental animal water. Specimens were then raised to post-hatching 
developmental stages (Nieuwkoop and Faber, 1994) under normal conditions. Once specimens reached the desired stage (i.e., when at least all elements of the chondrocranium were present as determined in Chapter 3), they were anesthetized by adding a few drops of 4\% MS-222 to their container. Once vital signs disappeared, the specimens were then fixed in $10 \% \mathrm{NBF}$ overnight at room temperature and then dehydrated for storage in a $70 \%$ ethanol solution at room temperature. Total specimen numbers for each treatment can be found in Appendix H.

\subsection{4: Bone and cartilage staining}

In order to visualize cartilage and bone morphology in control and experimental animals, specimens were subjected to standard clearing and staining methods. To visualize cartilage, specimens were placed in an Alcian blue (8GX, Acros Organics A3157) solution ( $20 \mathrm{~mL}$ acetic acid, $75 \mathrm{~mL}$ of $95 \%$ ethanol, $0.1 \mathrm{~g}$ alcian blue) for one to six hours, depending on the size of the specimen. Following this, specimens were moved through a hydration series (approximately one hour in each: 70\%, 50\%, 30\% ethanol, distilled water) before they were transferred into a trypsin solution (30\% saturated borate, $1 \%$ trypsin) and incubated at $37^{\circ} \mathrm{C}$ until their tissues were soft and approximately clear. Specimens were then transferred through a series of $1 \%$ potassium hydroxide $(\mathrm{KOH})$ plus glycerol solutions (approximately one hour to one day in each: 3:1, 1:1, and 1:3 $\mathrm{KOH}$ to glycerol) for further clearing and were finally stored in $100 \%$ glycerol.

To visualize bone development, specimens were placed into a 1\% Alizarin red S (Sigma-Aldrich A5533) in a $1 \% \mathrm{KOH}$ for one hour. The bone staining step is not size dependent. After bone staining, specimens were moved into a $30 \%$ saturated borate solution with $1 \%$ trypsin, and incubated at $37^{\circ} \mathrm{C}$, until they were almost completely clear. 
For storage and to finish clearing, the specimens were then transferred through a series of $1 \% \mathrm{KOH}$ plus glycerol solutions (approximately one hour to one day in each: $3: 1,1: 1$, and $1: 3 \mathrm{KOH}$ to glycerol) and stored in $100 \%$ glycerol.

\subsection{5: Nerve staining}

To visualize nerves as they relate to skeletal structures, the regressive nerve staining protocol outlined by Northcutt et al. (2000) was followed for both A. mexicanum and $X$. laevis specimens. Specimens were first bleached in a $10 \%$ hydrogen peroxide solution for one to two days, then washed in distilled water for two to three hours. Specimens were then macerated in a $3 \%$ trypsin $($ A. mexicanum) or $1 \%$ trypsin $(X$. laevis) in a $30 \%$ saturated borate solution until the tissues were clear and nerves were visible. To stain the nerves, specimens were then put in a $0.5 \% \mathrm{KOH}$ solution for ten minutes, $70 \%$ ethanol solution for ten minutes, then in a filtered 0.5\% Sudan Black B (Sigma-Aldrich 199664) dissolved in $70 \%$ ethanol solution for 30 minutes. To clear the excess Sudan Black B, specimens were washed in several $70 \%$ ethanol solutions until the nerves and tissues could be differentiated. Then, specimens were placed in a $0.5 \% \mathrm{KOH}$ solution overnight. For long term storage, specimens were transferred through a series of $1 \% \mathrm{KOH}$ plus glycerol solutions (approximately one hour in each: 3:1, 1:1, and 1:3 KOH to glycerol) and stored in $100 \%$ glycerol.

\subsection{6: Somite lineage tracing}

An unpublished dataset (courtesy of Maddin and Piekarski) of A. mexicanum specimens with GFP (green fluorescent protein)-positive somites grafted to confirm if homeotic transformations was obtained. Two clutches of embryos were generated for this dataset that were the result of a cross between a leucistic individual and a ubiquitous GFP- 
positive transgenic individual. Surgeries were performed on embryos between stages 21 and 27 (Bordzilovskaya et al., 1989). GFP-negative and GFP-positive embryos were first stage matched. The epidermis on the left side of both embryos was cut ventrally and peeled up, dorsally. The somite of interest was first removed from the host (GFP-negative) embryo. Next, the corresponding somite from the donor (GFP-positive) embryo was removed and it was placed within the space created in the host embryo. The epidermis was pulled back over the somites and held in place by a small piece of cover slip glass. Within an hour, host embryos had healed from the transplant surgery and were photographed for accuracy of the graft. Post-surgery embryos were then placed in autoclaved $40 \%$ Holtfreter's solution containing final concentrations of $0.01 \mu \mathrm{M}$ or $0.05 \mu \mathrm{M}$ retinoic acid or $20 \mu \mathrm{M}$ citral dissolved in DMSO solution. These specimens were left in their treatment for four, seven, or 14 days then removed from treatment and allowed to grow under normal conditions. Control specimens were placed in $40 \%$ Holtfreter's solution and an equal volume of DSMO alone as that added to experimental animal water.

Once specimens reached the desired stage (i.e., when skeletal structures of interest are present as determined in Chapter 3), they were anesthetized by adding a few drops of $4 \%$ MS-222 to their container and then once vital signs disappeared they were fixed in $4 \%$ paraformaldehyde overnight at $4{ }^{\circ} \mathrm{C}$. Specimens were then dehydrated through a methanol in phosphate buffered solution (PBS) series (one hour in each: 25\% methanol in PBS, 50\%, and $75 \%$ ) and stored in $100 \%$ methanol at $-20^{\circ} \mathrm{C}$. Specimens were later embedded in optimal cutting temperature (OCT) compound (Fisher Healthcare 23730571) by rehydrating the specimens through a methanol in PBS series (one hour in each: 75\% methanol in PBS, 50\% methanol in PBS, 25\% methanol in PBS, 100\% PBS). Specimens 
were then moved to a $15 \%$ sucrose in PBS solution and left overnight at $4{ }^{\circ} \mathrm{C}$, then placed in a $30 \%$ sucrose in PBS solution and left overnight at $4{ }^{\circ} \mathrm{C}$. Specimens were then left overnight for a third time in a 1:1 solution of $30 \%$ sucrose in PBS and OCT compound. The next day, specimens were mounted in an embedding mold filled with OCT compound and quickly frozen using a dry ice in $100 \%$ ethanol bath and stored at $-80^{\circ} \mathrm{C}$ to preserve fluorescence.

Embedded specimens were sectioned using a HM525 NX cryostat (Thermo Scientific) set to $-20^{\circ} \mathrm{C}$. Slices were 10 microns thick and resulting slides were stored at $80^{\circ} \mathrm{C}$ to preserve fluorescence. Sections were checked for fluorescence using a Zeiss Axio Imager.M2 microscope using the DSRed filter and photographed using a monochrome camera attachment. Fluorescence was artificially coloured green in the Zeiss Zen software.

\section{3: Results}

\subsection{1: Ambystoma mexicanum}

Many A. mexicanum specimens appeared to be developmentally normal based on external features, but when stained for cartilage or bone, it was clear that their internal development had been affected by their chemical treatments. Additionally, not every experimental specimen showed internal differences compared to controls. Entire Ambystoma mexicanum treatment groups were therefore batch whole-mount stained together, as it wasn't always obvious based on external features which specimens possessed modified internal morphologies. Sixty-one experimental specimens exposed to retinoic acid were cleared and stained, of those, thirty specimens showed modified internal morphologies (Appendix G, Table G1). Fifteen experimental specimens exposed to citral 
were cleared and stained and eleven specimens showed modified internal morphologies (Appendix G, Table G2).

\section{Exogenous retinoic acid experimental skull morphology}

In control specimens, the internasal plate is positioned posterior to Meckel's cartilage, whereas in experimental specimens at both dose levels (i.e., $0.01 \mu \mathrm{M}$ and 0.05 $\mu \mathrm{M}$ exogenous retinoic acid for either four or seven days) the internasal plate is positioned anterior to Meckel's cartilage (Figure 4.3). The anterior position was the result of the deformed internasal plate, but also a deformed Meckel's cartilage and palatoquadrate. Together, Meckel's cartilage and the palatoquadrate formed more rounded elements in experimental specimens compared to controls (Figure 4.3). The trabecular cartilage was also a much shorter element in experimental specimens at both dose levels compared to that of control specimens (Figure 4.3).

In the otic and post-otic region of the skull, the spatial relationship between the otic capsules and the ceratobranchials changed in experimental specimens exposed to higher doses of retinoic acid (Figure 4.3). In control specimens, the ventrally located ceratobranchials and dorsally located otic capsules align anteriorly (Figure 4.3A). In specimens exposed to lower doses of retinoic acid $(0.01 \mu \mathrm{M}$ retinoic acid for four or seven days; Figure 4.3B), this relationship did not change. However, in specimens exposed to higher doses of retinoic acid (Figure 4.3C), the first ceratobranchial was positioned posterior to the anterior limit of the otic capsule. The occipital arch became smaller in experimental specimens at both dose levels $(0.01 \mu \mathrm{M}$ or $0.05 \mu \mathrm{M}$ exogenous retinoic acid for four or seven days) compared to control specimens (Figure 4.3). In experimental specimens exposed to lower doses of retinoic acid (e.g., $0.01 \mu \mathrm{M}$ retinoic acid for four or 
seven days), the occipital arch would often be present as a smaller element. Either a completely or incompletely separate additional neural arch also occurred posterior to the occipital arch (Figure 4.3B).

The axial skeleton became important to examine in experimental specimens of $A$. mexicanum. As the shoulder girdle of $A$. mexicanum develops soon after hatching (Nye et al., 2003), it was a reliable indicator of how many segments had been transformed from occipital elements to axial elements (Figure 4.3). In control specimens, the shoulder girdle was consistently located between the first and second neural arches (Figure 4.3A). In specimens exposed to lower doses of retinoic acid (e.g., $0.01 \mu \mathrm{M}$ retinoic acid for four or seven days), the shoulder girdle was often present between the second and third neural arches (Figure 4.3B). In some specimens exposed to higher doses of retinoic acid (e.g., $0.05 \mu \mathrm{M}$ retinoic acid for four or seven days), the shoulder girdle was present between the third and fourth neural arches (Figure 4.3C). 


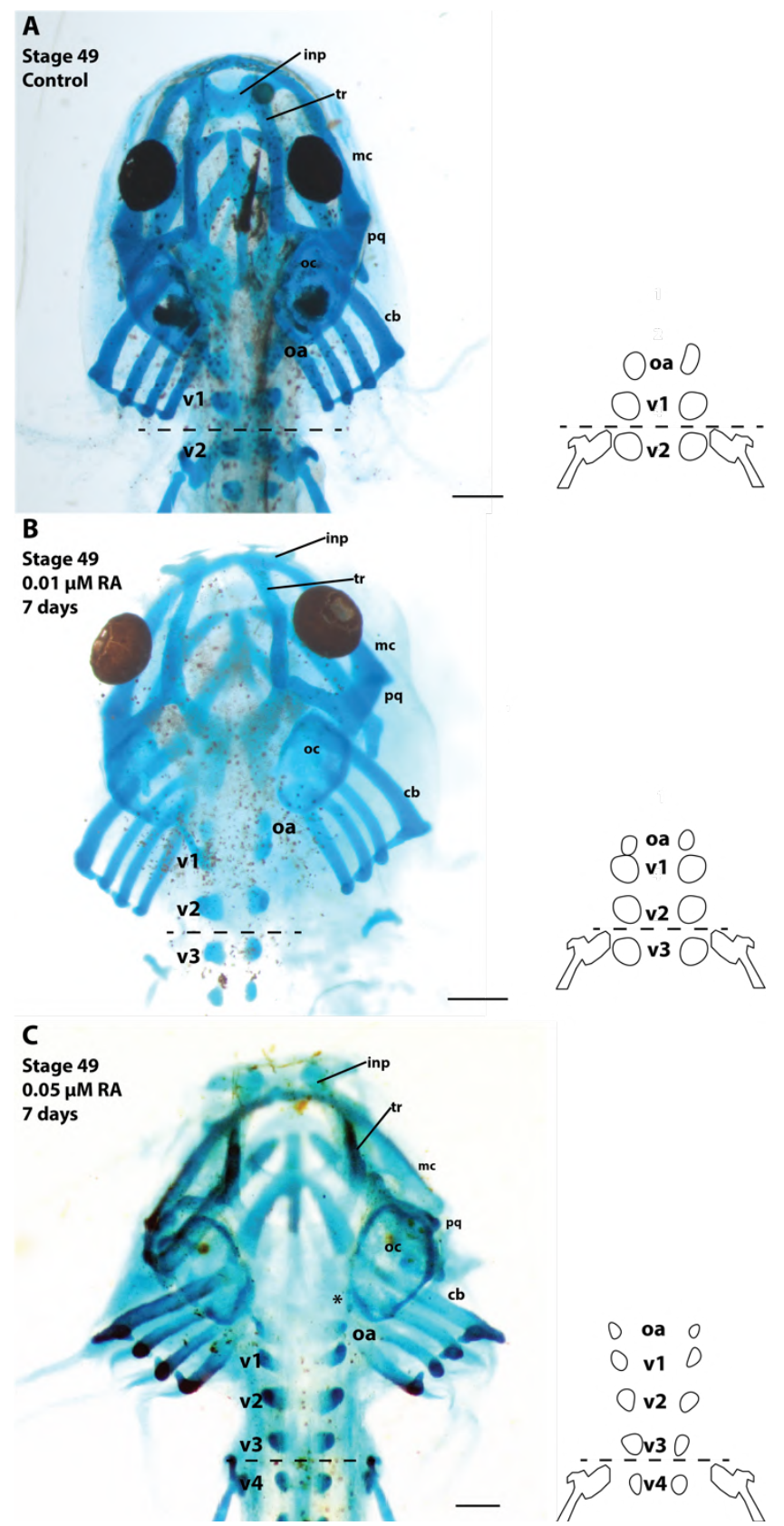

Figure 4.3. Ambystoma mexicanum specimens exposed to exogenous retinoic acid. Schematics to the right help to interpret the number of vertebral elements visible anterior to the shoulder girdle. A) A stage 49 control specimen with cartilage visible. The shoulder girdle is positioned between the first and second vertebral elements (dashed line). B) A stage 49 specimen exposed to $0.01 \mu \mathrm{M}$ retinoic acid for four days. The shoulder girdle is positioned between the second and third vertebral elements. C) a stage 49 specimen exposed to $0.05 \mu \mathrm{M}$ retinoic acid for seven days. The shoulder girdle is positioned between the third and fourth vertebral elements. There is a possible additional element near the otic capsule (oc), indicated by an asterisk. Scale bars equal $5 \mathrm{~mm}$. Abbreviations: cb, ceratobranchials; inp, internasal plate; mc, Meckel's cartilage; pq; palatoquadrate; oa, occipital arch; tr, trabecular cartilage; v1-4, first through fourth vertebral elements. 
Some specimens exposed to lower doses of retinoic acid for shorter periods of time (e.g., $0.01 \mu \mathrm{M}$ retinoic acid for four days) survived the treatment well and developed until ossification was present in their skull (Figure 4.4). While these specimens did not exhibit the same cartilage abnormalities as in younger specimens exposed to higher doses of retinoic acid, they often had abnormal frontal, parietal, and premaxilla elements. The alary process of the premaxilla was longer and thinner in experimental specimens compared to control specimens (Figure 4.4). The frontal and parietal elements were wavy instead of straight along their lateral extents (Figure 4.4).
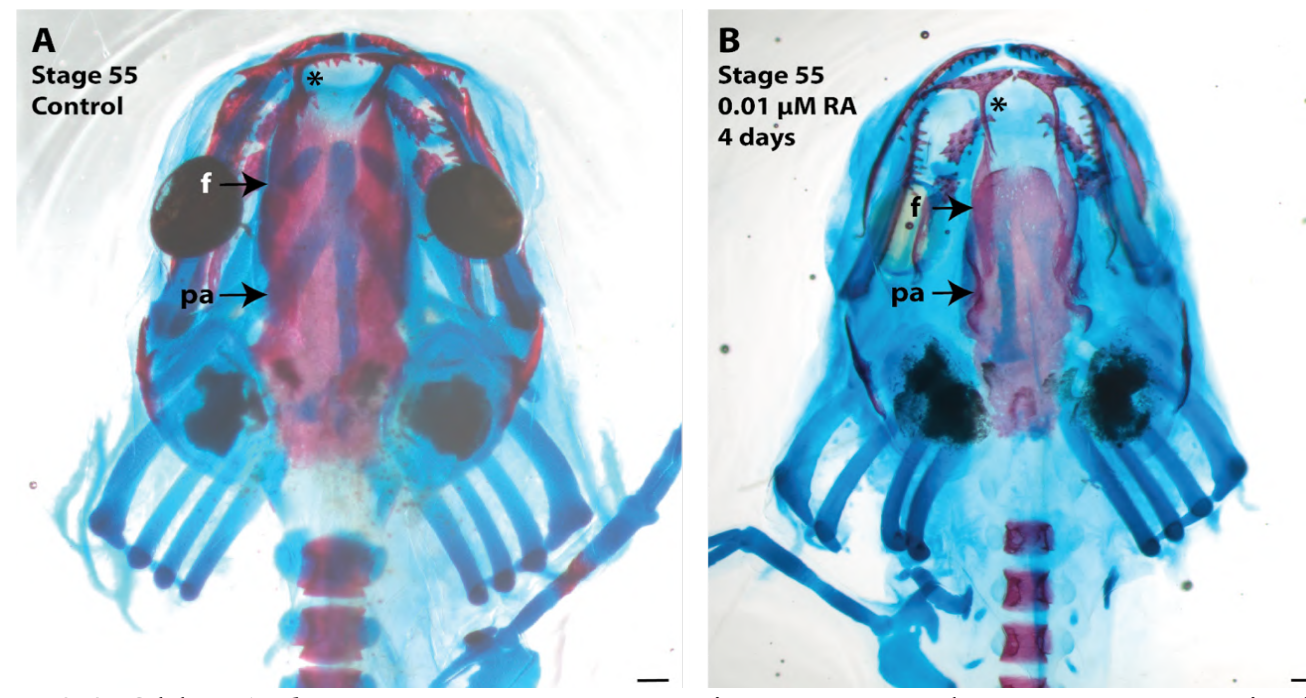

Figure 4.4. Older Ambystoma mexicanum specimens exposed to exogenous retinoic acid. A) A stage 55 control with bone (red) and cartilage (blue) visible. The frontal (f) and parietal (pa) bones are relatively straight. B, a stage 55 specimen that was exposed to 0.01 $\mu \mathrm{M}$ retinoic acid for four days. The frontal and parietal bones are wavy (arrows). In B) the premaxilla has also changed shape (asterisk) compared to the morphology in A. Scale bars equal $5 \mathrm{~mm}$.

\section{Citral experimental skull morphology}

In the anterior, pre-otic region the morphology of the cartilaginous elements present in the skull did not change between control and any experimental specimens (Figure 4.5). In the post-otic region of the skull, the parachordal cartilage was longer in experimental 
specimens (Figure 4.5). In control specimens, the posterior limit of the parachordal cartilage was located anterior to the posterior limit of the otic capsule (Figure 4.5A). In experimental specimens exposed to the highest doses of citral (e.g., $20 \mu \mathrm{M}$ citral), the posterior limit of parachordal cartilage was located posterior to the posterior limit of the otic capsule (Figure 4.5C).

In experimental specimens, cartilage linking the occipital arch to the first neural arch (Figure 4.7B, C) and to the parachordal cartilage (Figure 4.5C) was present. This cartilage was rarely present, but when present appeared in specimens exposed to the highest doses of citral (e.g., $10 \mu \mathrm{M}$ or $20 \mu \mathrm{M}$ citral). 


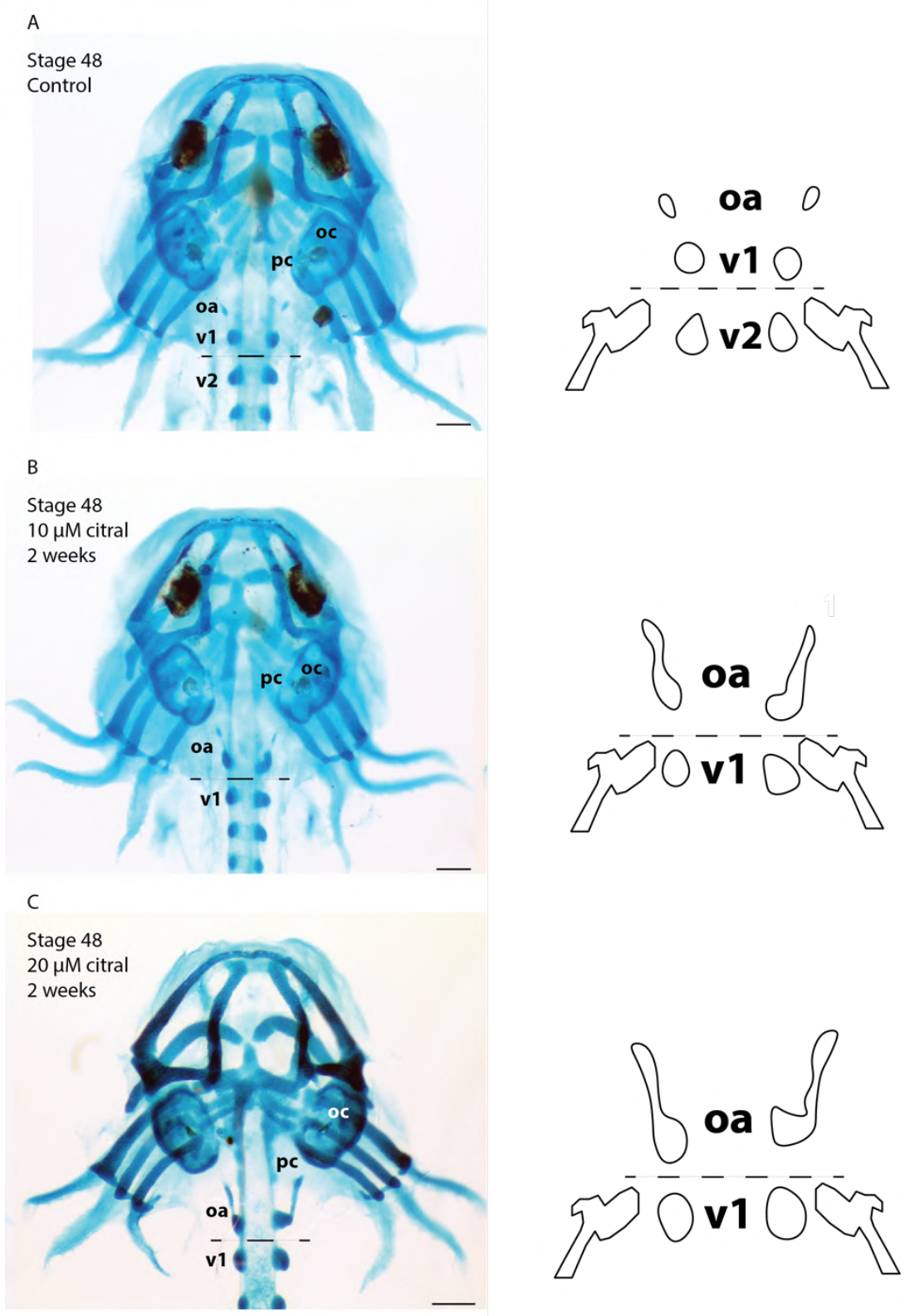

Figure 4.5. Ambystoma mexicanum specimens where normal retinoic acid expression within the embryo was inhibited with citral. Schematics to the right help to interpret the number of vertebral elements visible anterior to the shoulder girdle. A) A stage 48 control with cartilage visible. The skull-neck boundary (dashed line) is positioned between the occipital arch (oa) and the first vertebral element (v1). The shoulder girdle is located between the first and second (v2) vertebral elements. B) A stage 48 specimen exposed to $10 \mu \mathrm{M}$ citral for two weeks. The first vertebral element is connected to the skull via cartilage between it and the occipital arch. The shoulder girdle is now associated with the first vertebral element located outside of the head. C) A stage 48 specimen exposed to 20 $\mu \mathrm{M}$ citral for two weeks. The cartilaginous link between the occipital arch and first vertebral element is stronger. The shoulder girdle is now associated with the first vertebral element located outside of the head. Scale bars equal $5 \mathrm{~mm}$. Abbreviations: oc, otic capsule; pc, parachordal cartilage. 
The specimens did not tolerate the citral treatment well; however, some specimens developed until ossification was present in their skull (Figure 4.6). As in specimens exposed to exogenous retinoic acid, the alary process of the premaxilla was elongated and thinner in experimental specimens compared to control specimens. The overall shape of the skull changed in the post-otic region of the skull, as in experimental specimens the region was wider than in control specimens (Figure 4.6).

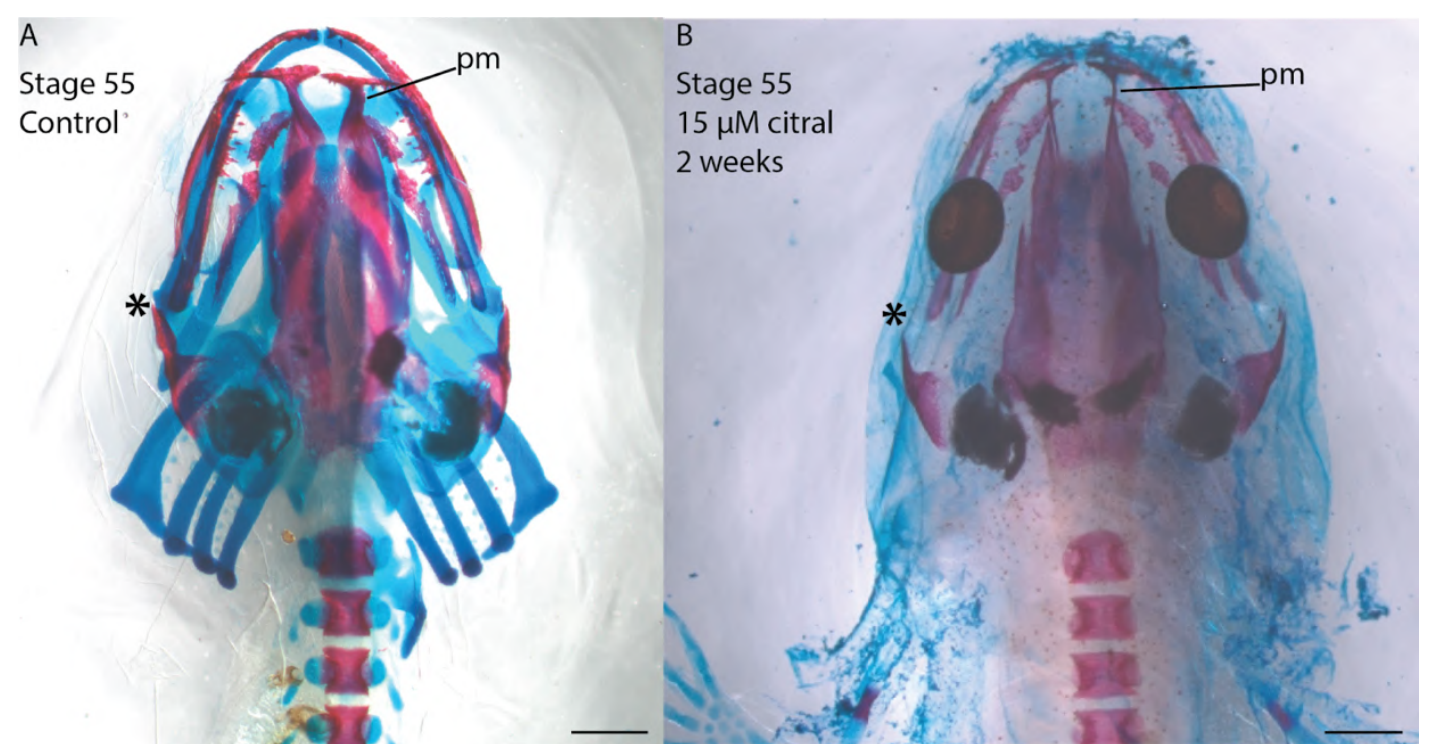

Figure 4.6. Older Ambystoma mexicanum specimens where normal retinoic acid synthesis within the embryo was inhibited with citral. A) A stage 55 control cartilage (blue) and bone (red) stained. The premaxilla ( $\mathrm{pm}$ ) and the shape of the back of the head (asterisk) are normal. B) A stage 55 specimen exposed to $15 \mu \mathrm{M}$ citral for two weeks. The alary process of the premaxilla is much longer than observed in control specimens. The back of the head is rounder than observed in control specimens. Scale bars equal $5 \mathrm{~mm}$.

\section{Experimental nerve morphology}

A subset of experimental specimens exposed to exogenous retinoic acid and citral were stained to visualize nerves. Unfortunately, there were no morphological differences between the control and experimental specimens with respect to the relative location of the hypoglossal nerve. Five specimens exposed to exogenous retinoic acid were nerve stained and two specimens exposed to citral (Appendix G). In all specimens nerve stained and 
examined, the hypoglossal nerve complex passed laterally through the axial skeleton between the occiput and the first vertebral element, regardless of treatment received (Figure 4.7). As in control specimens, three roots were present in experimental specimens. Notably, the skeletal morphology of these specimens was also unchanged.
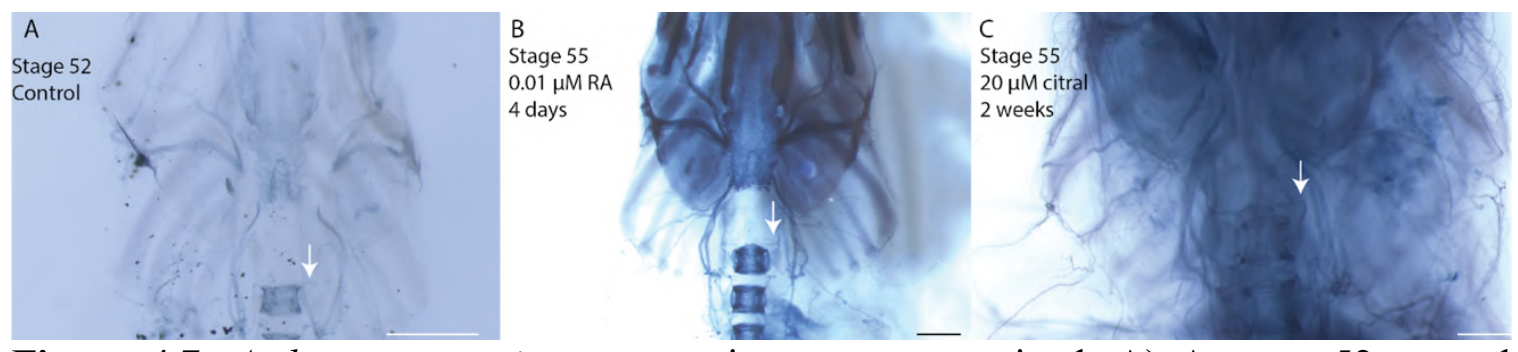

Figure 4.7. Ambystoma mexicanum specimens, nerve stained. A) A stage 52 control specimen where the hypoglossal nerve nerve passes laterally through the axial skeleton between the occiput and first vertebral element (arrow). B) A stage 55 specimen exposed to $0.01 \mu \mathrm{M}$ exogenous retinoic acid for four days. The hypoglossal nerve again passes laterally through the axial skeleton between the occiput and first vertebral element. C) A stage 55 specimen exposed to $20 \mu \mathrm{M}$ citral for two weeks. The hypoglossal nerve again passes laterally through the axial skeleton between the occiput and first vertebral element. Scale bars equal $1 \mathrm{~mm}$.

\section{Experimental somite cell-lineage tracing}

Somite cell-lineage tracing experiments were conducted on A. mexicanum specimens to determine the occurrence of homeotic transformations. In whole-mount specimens that were exposed to exogenous retinoic acid, morphological changes in muscle fate were apparent. In somite two grafted control specimens, GFP-labelling is present in the $\mathrm{m}$. geniohyoideus and the $\mathrm{m}$. rectus cervicis (Figure 4.8A, left panel). In a somite two grafted specimen exposed to $0.05 \mu \mathrm{M}$ exogenous retinoic acid for two weeks, GFP-label was no longer observed in the m. geniohyoideus (Figure 4.8A, middle panel); however, label was still present in the $\mathrm{m}$. rectus cervicis and additionally in a portion of the first hypaxial trunk muscle (Figure 4.8A, middle panel). The location of the GFP-label in this specimen exposed to exogenous retinoic acid is similar to the location of the GFP-label observed in specimens where somite three was grafted (Figure 4.8A, right panel). 
In whole-mount specimens where somite three was grafted GFP-labelling was present in the $\mathrm{m}$. rectus cervicis and additionally in part of the first hypaxial trunk muscle (Figure 4.8A right panel, B left panel). In a specimen where somite three was grafted and then exposed to $0.01 \mu \mathrm{M}$ exogenous retinoic acid for four days, the $\mathrm{m}$. rectus cervicis was no longer GFP-labelled. The hypaxial trunk musculature was still GFP-labelled, and additionally the epaxial musculature was GFP-labelled (Figure 4.8B, middle panel). The location of the GFP-label in the specimen exposed to exogenous retinoic acid is similar to the location of the GFP-labelling observed in specimens where somite four was grafted (Figure 4.8B, right panel). 


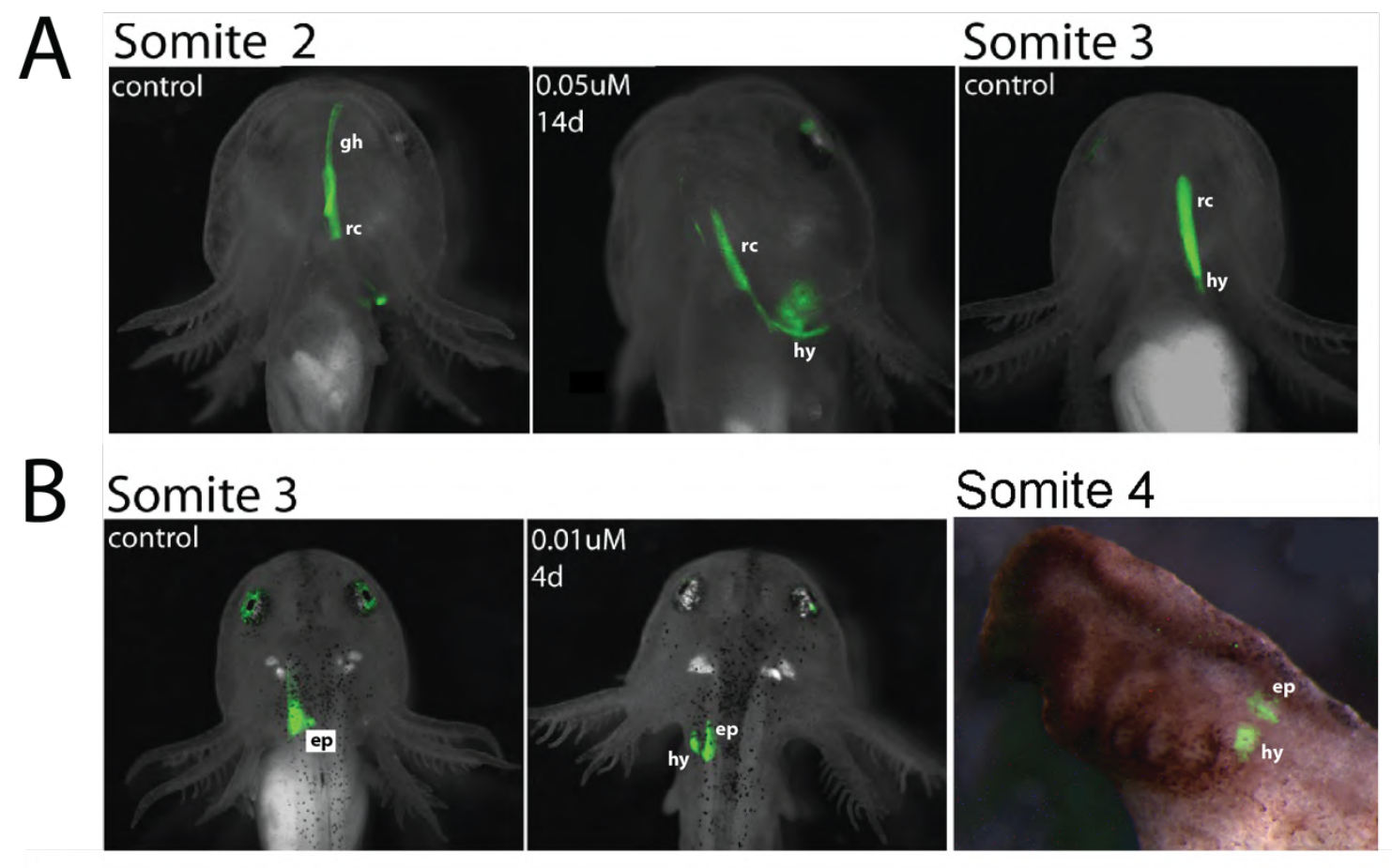

Figure 4.8. Unpublished dataset (courtesy of Maddin and Piekarski) of whole-mount Ambystoma mexicanum specimens with GFP-transplanted somites. Some experimental specimens were exposed to retinoic acid after somite transplantation to determine if homeotic transformations of somites had occurred. A) A control specimen in ventral view with somite two grafted (left) compared to a somite two grafted experimental specimen (middle) in ventral view that was exposed to $0.05 \mu \mathrm{M}$ exogenous retinoic acid for two weeks. The GFP-labelled muscles resemble those found in a normal somite three grafted specimen (right), this specimen is also in ventral view. B) A control specimen with somite three grafted (left) in dorsal view compared to a somite three grafted experimental specimen (middle), also in dorsal view, that was exposed to $0.01 \mu \mathrm{M}$ exogenous retinoic acid for four days. The GFP-labelled muscles resemble those found when somite four is grafted (right), specimen in lateral view. Abbreviations: ep, epaxial; gh, m. geniohyoideus; hy, hypaxial trunk musculature; rc, m. rectus cervicis.

In addition to the whole-mount specimens, additional GFP-labelled specimens, where somite three was grafted, were sectioned to better observe the contribution of the GFP-positive cells to the skull and axial skeleton. In control specimens, sections revealed weak GFP-label present in the posterior portion of the occipital arch and in the anterior portion of the first vertebral element (Figure 4.9A). Stronger GFP-label was present in the muscles due to higher abundance of cells in that tissue, in two dorsally located myotomes, 
and in the ventrally located $\mathrm{m}$. rectus cervicis and the first hypaxial trunk musculature (Figure 4.9A).

In a specimen where somite three was grafted then exposed to $0.05 \mu \mathrm{M}$ exogenous retinoic acid for four days, the position of the GFP-label changed in comparison to the control. Weak GFP-labelling is present in the posterior portion of the first vertebral element and in the anterior portion of the second vertebral element (Figure 4.9B). Stronger GFPlabel is again present in the musculature. Two myotomes are GFP-labelled, as in the control specimen (Figure 4.9A, B). Ventrally, the hypaxial musculature is GFP-labelled (Figure 4.9B).

A third specimen where somite three was grafted and then exposed to $20 \mu \mathrm{M}$ of citral for two weeks was also sectioned and examined. In this specimen, GFP-label was not present in the axial skeleton at all. There was a weak GFP-label in the anterior portion of the occipital arch, and none in the posterior portion of the arch. A stronger GFP-label was seen in only one myotome (Figure 4.9C). 
A Somite 3: control

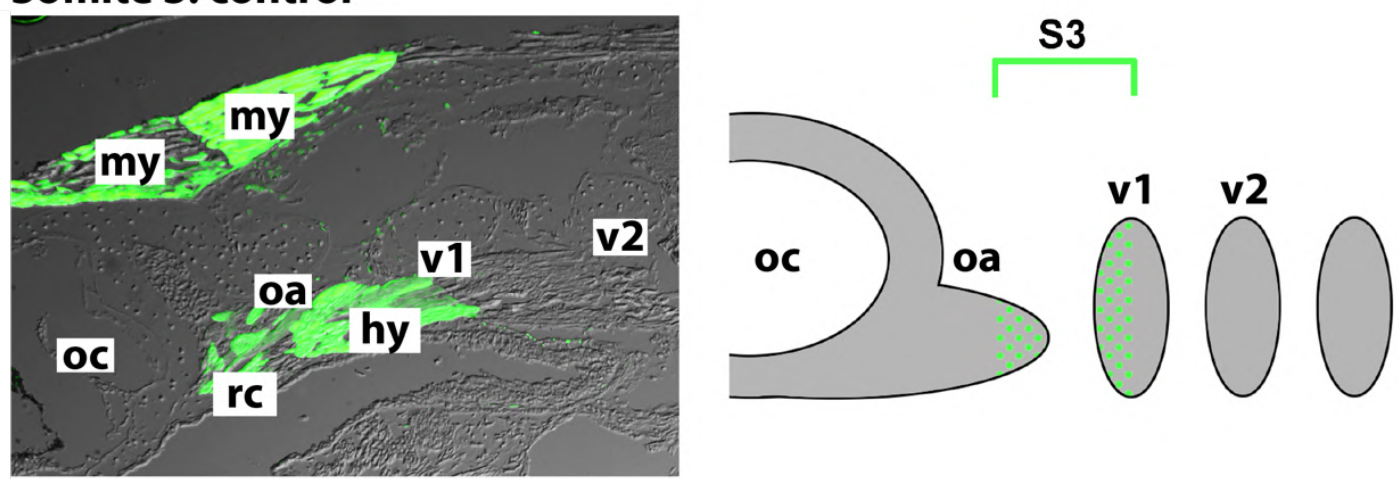

B Somite 3: $0.05 \mu \mathrm{M}$ retinoic acid for 4 days
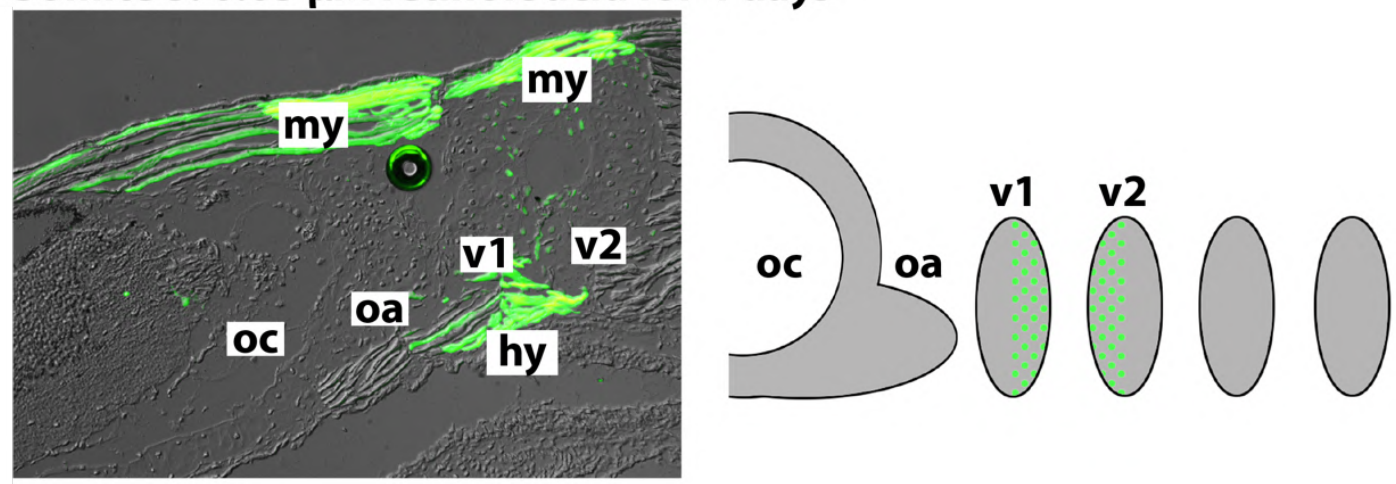

\section{Somite 3: $20 \mu \mathrm{M}$ citral for two weeks}
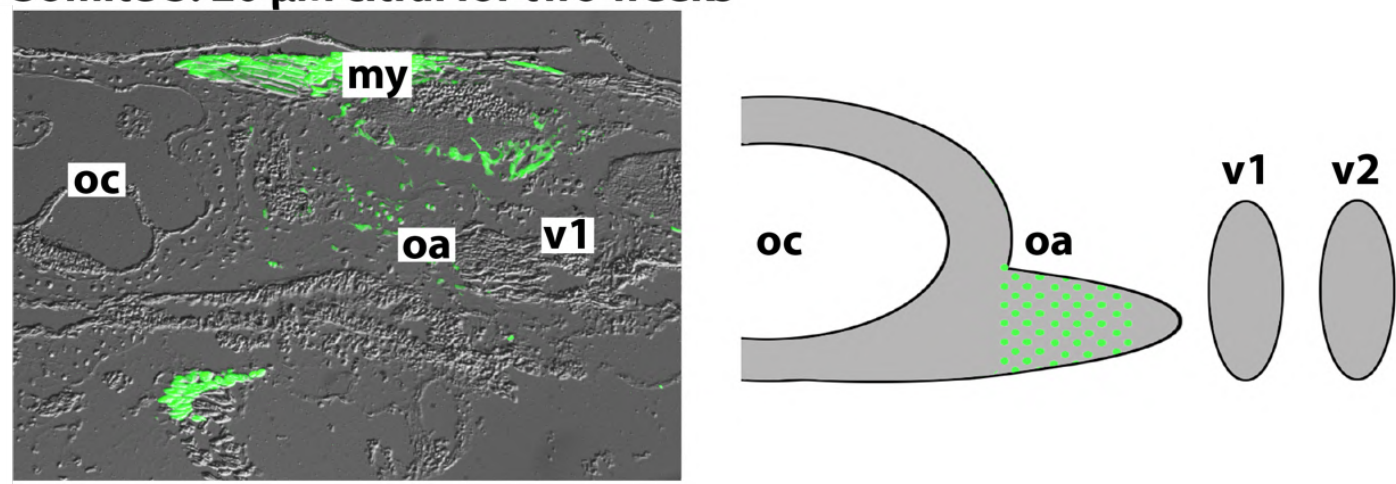

Figure 4.9. Unpublished dataset (courtesy of Maddin and Piekarski) of sectioned Ambystoma mexicanum specimens with GFP-transplanted somites. Each photographed section (left) has an interpretive schematic (right). A) A control specimen with somite three transplanted shows GFP-positive label in the posterior portion of the occipital arch and in the anterior portion of the first vertebral element. B) A specimen with a transplanted somite three that was then exposed to $0.05 \mu \mathrm{M}$ exogenous retinoic acid for four days shows GFPpositive label in the posterior portion of the first vertebral element and in the anterior portion of the second. C) A specimen with somite three transplanted that was then exposed to $20 \mu \mathrm{M}$ citral, a retinoic acid inhibitor, for two weeks shows label entirely in the occipital arch and not in the vertebral elements. Abbreviations: ep, epaxial; gh, m. geniohyoideus; hy, hypaxial trunk musculature; oa, occipital arch; oc, otic capsule; my, myomere; rc, m. rectus cervicis; v1, first vertebral element; v2, second vertebral element. 


\subsection{2: Xenopus laevis}

\section{External morphology}

Previous research in exogenous retinoic acid and citral conducted in $X$. laevis revealed important external features that I could use to select specimens for cartilage, nerve, and bone staining (Figure 4.10; Ruiz i Altaba and Jessel, 1991; Schuh et al., 1993). Previous research showed that exogenous retinoic acid causes an increased number of melanophores (Figure 4.10B; Ruiz i Altaba and Jessel, 1991), whereas other research showed that inhibiting retinoic acid via citral causes a more rounded head in experimental specimens and a ventral bulge in the area of the hyobranchial apparatus (Figure 4.10D, F; Schuh et al., 1993). Not every experimental specimen exhibited these external features. Only specimens that had these external features were selected for cartilage, bone, and nerve staining analyses as they were the most promising to reveal an altered morphology. Twenty-one experimental specimens exposed to retinoic acid were cleared and stained, of those, seventeen specimens showed modified internal morphologies (Appendix H, Table H1). Twenty-three experimental specimens exposed to citral were cleared and stained and fifteen specimens showed modified internal morphologies (Appendix H, Table H2). 


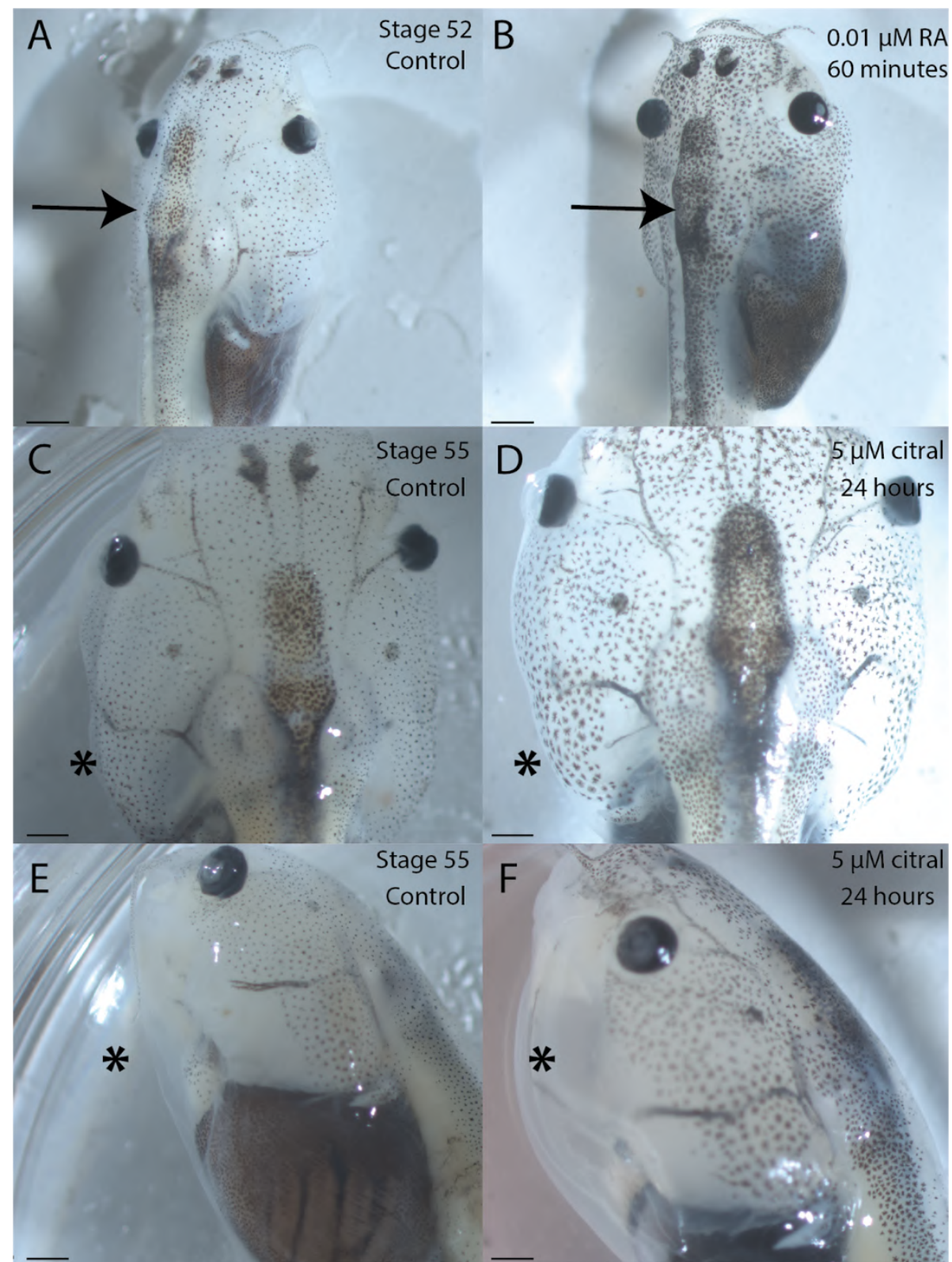

Figure 4.10. Xenopus laevis experimental specimens external morphology compared to the morphology of the control specimens. Exogenous retinoic acid and inhibiting retinoic acid expression causes visible, morphological changes to the external features of $X$. laevis. A) A stage 52 control specimen in dorsolateral view. B) A stage 52 experimental specimen in dorsolateral view exposed to $0.01 \mu \mathrm{M}$ exogenous retinoic acid for 60 minutes during somite formation. The experimental specimen has darker pigmentation throughout (arrow). C) A stage 55 control in dorsal view. D) A stage 55 experimental specimen in dorsal exposed to $5 \mu \mathrm{M}$ citral for 24 hours. The experimental specimen has a more rounded head in the ventral region (asterisk). E) A stage 55 control in ventrolateral view. F) A stage 55 experimental specimen in dorsolateral view exposed to $5 \mu \mathrm{M}$ citral for 24 hours. The hyobranchial apparatus of the experimental specimen protrudes more ventrally than in the control (asterisk). Scale bars equal $1 \mathrm{~mm}$. 


\section{Exogenous retinoic acid experimental skull morphology}

X. laevis specimens exposed to exogenous retinoic acid starting at stage 10 for 30 minute pulses or left overnight for seventeen hours did not show morphological differences (data not shown), however, survivability was much higher when specimens were exposed to exogenous retinoic acid for only 60 minutes (Appendix H). For example, 30 out of 60 specimens survived the $0.01 \mu \mathrm{M}$ retinoic acid treatment for 60 minutes, versus eight out of 120 specimens that survived the overnight treatment (Appendix $\mathrm{H}$ ).

In X. laevis specimens, the morphological changes to the post-otic portion of the skull were not evident until specimens started to develop neural arches at stage 50-51 (Figure 4.11). In younger specimens, morphological abnormalities to the hyobranchial apparatus were evident (Figure $4.11 \mathrm{~A}, \mathrm{~B}$ ). In these specimens, the ceratobranchials, pharyngeal clefts, and commissura terminalis were much less robust than observed in control specimens (Figure 4.11A, B). The commissura terminalis, the element that unites the four ceratobranchials distally, as well the ceratobranchials, were very fragile elements in experimental specimens with a smaller surface area than observed in controls (Figure 4.11A, B).

Once specimens reached developmental stage 50-52, when the neural arches were first present, morphological changes to the post-otic region of the skull became evident. In the occipital arch, the exoccipital foramen was much larger in experimental specimens than observed in control specimens (Figure 4.11C, D). This is a result of a less robust occipital arch in experimental specimens (Figure 4.11D). In control specimens, the first neural arch is positioned immediately posterior to the occipital arch (Figure 4.11C). In experimental 
specimens, a large gap was consistently present between the occipital arch and the first neural arch (Figure 4.11D).

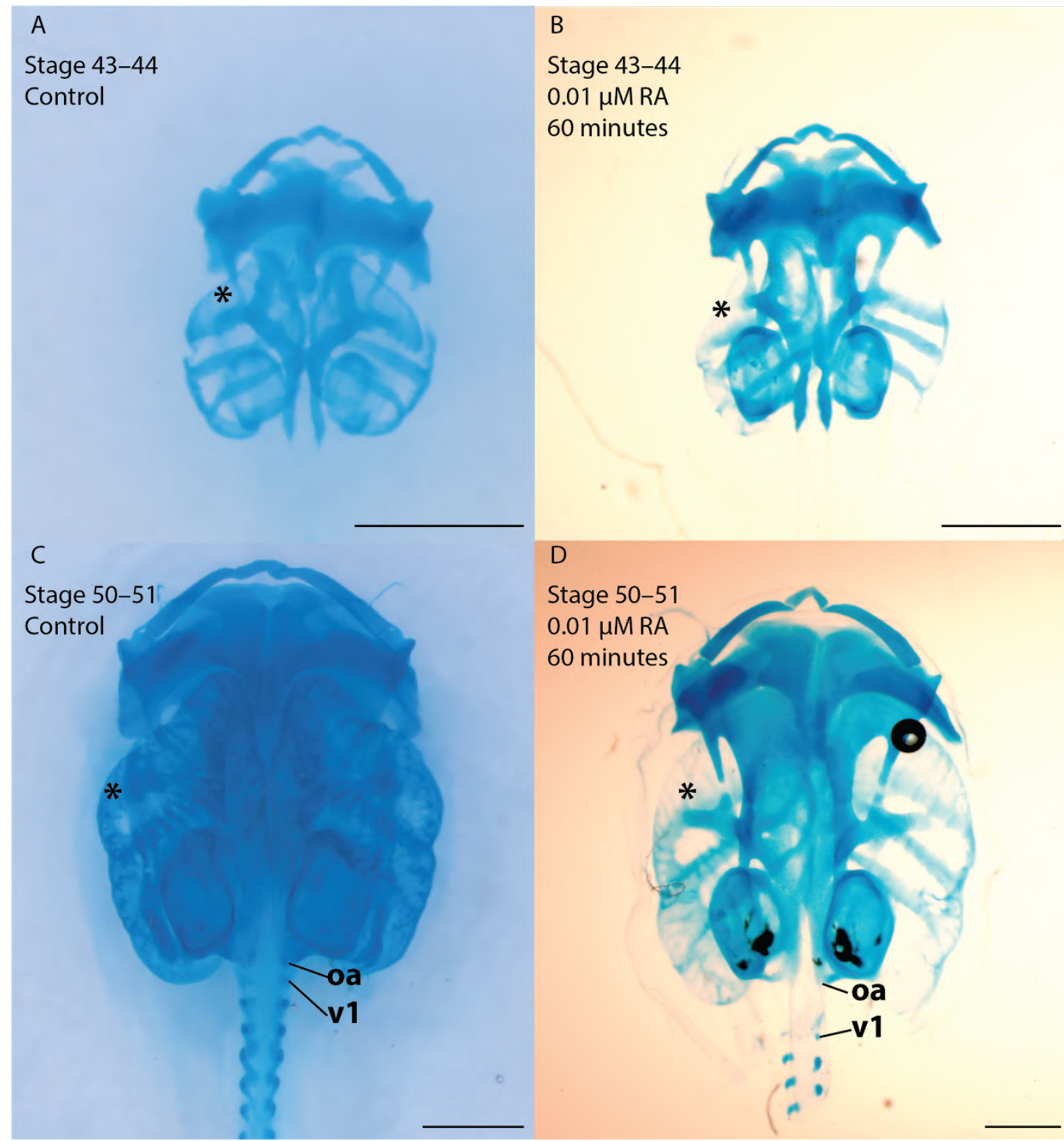

Figure 4.11. Xenopus laevis specimens exposed to exogenous retinoic acid. A) A stage $43 / 44$ control specimen. B) A stage 43-44 experimental specimen exposed to $0.01 \mu \mathrm{M}$ retinoic acid for 60 minutes starting at stage 10 . The four ceratobranchials, pharygenal clefts, and commissura terminalis are less robust (asterisk) than in control specimens. C) A stage 50/51 control specimen. D) A stage 50-51 experimental specimen exposed to 0.01 $\mu \mathrm{M}$ retinoic acid for 60 minutes starting at stage 10 . In the older experimental specimen, the ceratobranchials and commissura terminalis are again less robust than in control specimens. The jugular foramen of the occipital arch (oa) is much larger than in control specimens, and occipital arch as a whole is less robust in experimental specimens. Additionally, there is now a large gap between the occipital arch and the first vertebral element (v1). Scale bars equal $1 \mathrm{~mm}$. 


\section{Citral experimental skull morphology}

Xenopus laevis specimens exposed to citral and then stained with alcian blue for cartilage were difficult to interpret. The experimental specimens had more robust cartilage than observed in control specimens (data not shown) and it was difficult to assess cartilage at the occipital arch. In developmentally older specimens stained with Sudan Black B, which stains phospholipids and intracellular lipids and allows one to visualize nerves and bone (Filipski and Wilson, 1984), the occipital arch was easier to observe (Figure 4.12).

In control specimens, the first vertebral element is positioned quite close to the occipital arch, but these elements are separate (Figure 4.12A). In experimental specimens exposed to doses of citral at or higher than $2.5 \mu \mathrm{M}$, the first vertebral element was often attached to the posterior edge of the occipital arch and became one element confluent with the occipital arch (Figure 4.12B). In experimental specimens with this morphology the first true vertebral element (the second vertebral element in control specimens) was always positioned quite close to the occipital arch (Figure 4.12B). This morphology is visible in control specimens between the occipital arch and the first vertebral element (Figure 4.12A). The more posterior vertebral elements were then spaced evenly apart. 

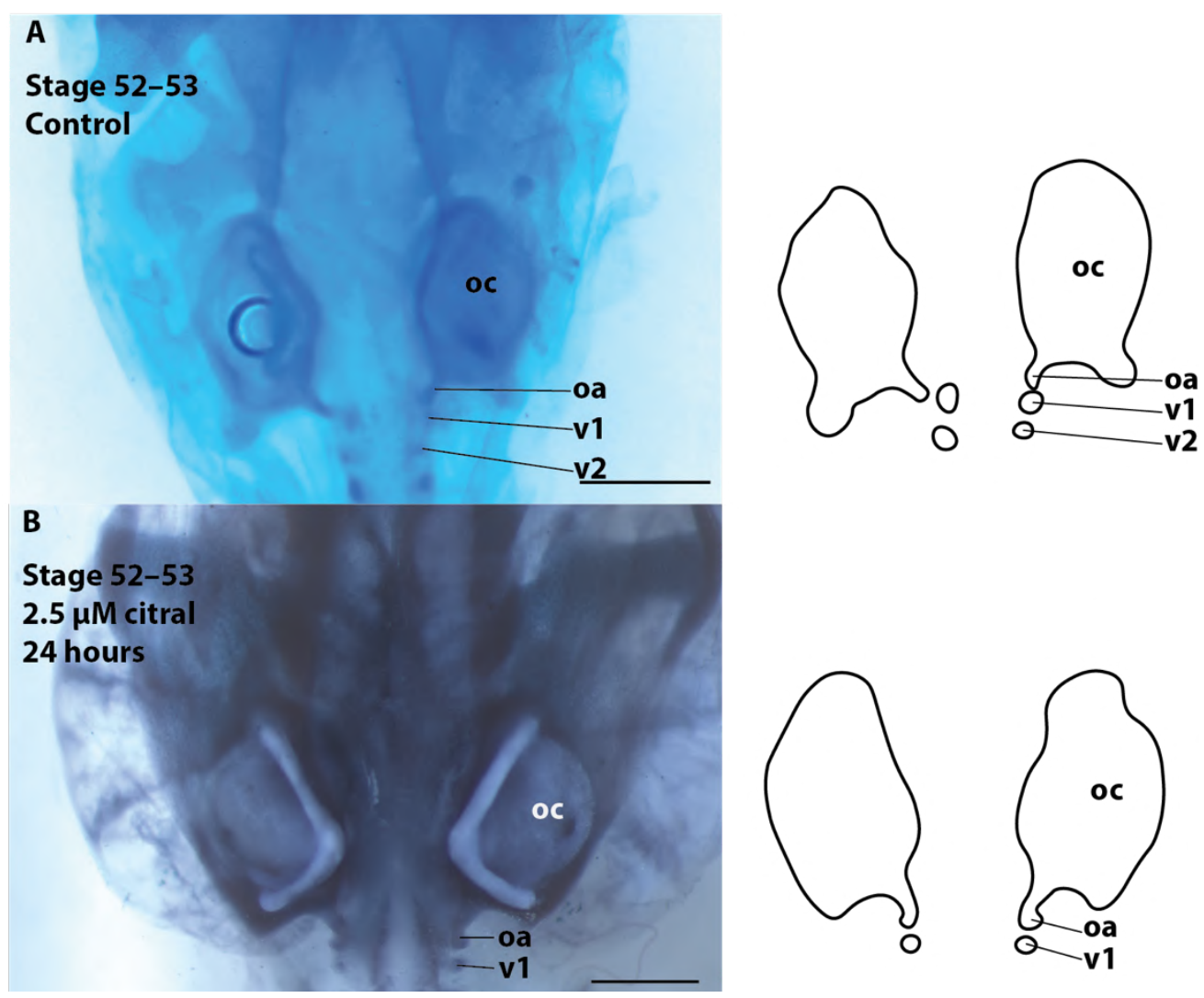

Figure 4.12. Xenopus laevis where normal retinoic acid expression within the embryo was inhibited with citral. Citral experimental specimens are whole-mount stained for cartilage A) and for nerves (B) (note cartilage is also stained in B). A) A stage 52-53 control showing that while the occipital arch (oa) and the first vertebral element (a1) are closely associated but are separate elements. B) A stage 52-53 experimental specimen treated with $2.5 \mu \mathrm{M}$ citral for 24 hours. In B, the first vertebral element is attached to the occipital arch and the second vertebral element is now the first. Scale bars equal $1 \mathrm{~mm}$. Line drawings to help interpret structures on the right. Dashed lines indicate the skull-neck boundary. Abbreviations: Oc, otic capsule; v1, first vertebral element; v2, second vertebral element.

\section{Experimental nerve morphology}

Unfortunately, $X$. laevis specimens exposed to exogenous retinoic acid did not stain well with Sudan Black B nerve staining protocols. However, a subset of experimental specimens exposed to citral $(\mathrm{n}=6)$ were stained to visualize nerves. In control $X$. laevis specimens, there was some variation among control specimens in where the hypoglossal nerve complex passed through the axial skeleton (see Chapter 3). In some control specimens, the hypoglossal nerve passed laterally between the first and second vertebral 
elements (Figure 3.13C, D; Figure 4.13A). In other specimens, the hypoglossal nerve was observed to pass laterally between the first and second, and additionally between the second and third vertebral elements (Figure 3.13C). In X. laevis specimens exposed to citral, the hypoglossal nerve was observed to pass between the occipital arch and the first vertebral element in all specimens (Figure 4.13B).
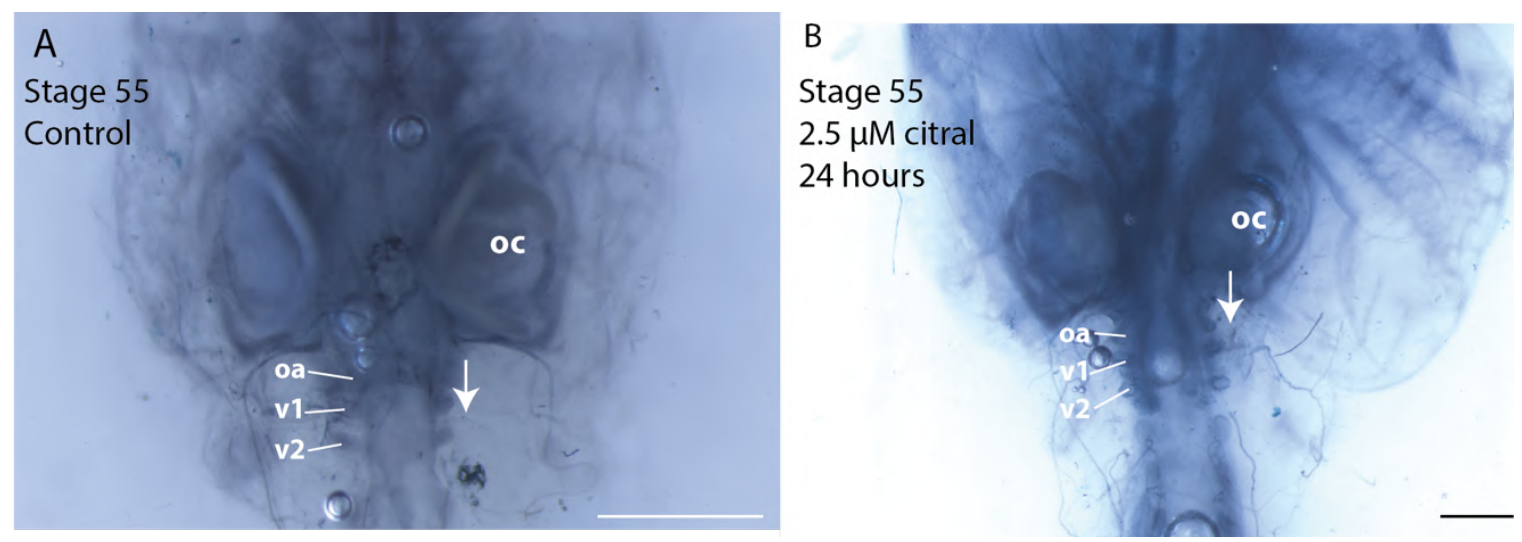

Figure 4.13. Xenopus laevis specimens, stained with Sudan Black B to visualize nerves. A) A stage 55 control specimen, the hypoglossal nerve (arrow) passes laterally through the axial skeleton between the first (v1) and second (v2) vertebral elements. B) In an experimental specimen exposed to $2.5 \mu \mathrm{M}$ for 24 hours, the hypoglossal nerve (arrow) passes laterally through the axial skeleton between the occipital arch (oa) and the first vertebral element. Scale bars equal $1 \mathrm{~mm}$. Other abbreviations: oc, otic capsule.

\section{4: Discussion}

The goal of the present study was to test the hypothesis that a shift in Hox gene expression domains can cause homeotic transformations at the occiput in A. mexicanum and $X$. laevis. To test this hypothesis, I manipulated Hox gene expression domains by administering exogenous retinoic acid to and inhibiting retinoic acid in A. mexicanum and $X$. laevis embryos during somitogenesis (A. mexicanum and X. laevis) and before somitogenesis (X. laevis) with the prediction that this would cause homeotic transformation of somites at the skull-neck boundary. Applying exogenous retinoic acid or inhibiting the oxidation of retinol to retinoic acid via citral did confirm the ability to induce homeotic 
transformation of somites in some experimental specimens, as shown with nerve staining analysis and GFP-grafting experiments.

This study is the first in which morphological changes in the skulls of A. mexicanum and $X$. laevis that were exposed to either exogenous retinoic acid or a retinoic acid inhibitor were observed and described for these species. The number of extraneous neural arches that appeared or neural arches attached to the skull are good indicators of homeotic transformation of somite fate. In amniotes, five somites generally contribute to the occipital arch or arches (Figure 4.14; de Beer, 1937; Couly et al., 1993; Huang et al., 2000; Maddin et al., 2020). For our lissamphibian model organisms, three somites contribute to the occiput in A. mexicanum (Figure 4.14; Piekarski and Olsson, 2007) and two somites are hypothesized to contribute to the occiput in X. laevis (Figure 4.14; de Beer 1937; Maddin et al., 2020). When A. mexicanum specimens were exposed to exogenous retinoic acid, lower doses of retinoic acid (e.g., $0.01 \mu \mathrm{M})$ caused the appearance of one additional neural arch anterior to the first vertebra (Figure 4.3B), and a higher dose of retinoic acid (e.g., $0.05 \mu \mathrm{M}$ ) caused two additional neural arches to appear (Figure 4.3C). Conversely, when A. mexicanum was exposed to the retinoic acid inhibitor citral, the first vertebral element became connected to the occiput via a cartilaginous bridge, effectively transforming a formerly vertebral element into an exoccipital (Figure 4.5B, C). These observations are consistent with the prediction that when retinoic acid is applied to A. mexicanum embryos single somites transform from occipital to vertebral fates. The result when a single additional neural arch appears is interpreted as indicating one fewer somite contributing to the occiput in A. mexicanum, thereby replicating the condition present in normal development of $X$. laevis in the case of low dose exogenous retinoic acid application. 
Conversely, when exposed to a retinoic acid inhibitor, a vertebral somite is added to the occiput, thereby approaching the amniote condition (Figure 4.14). 
A
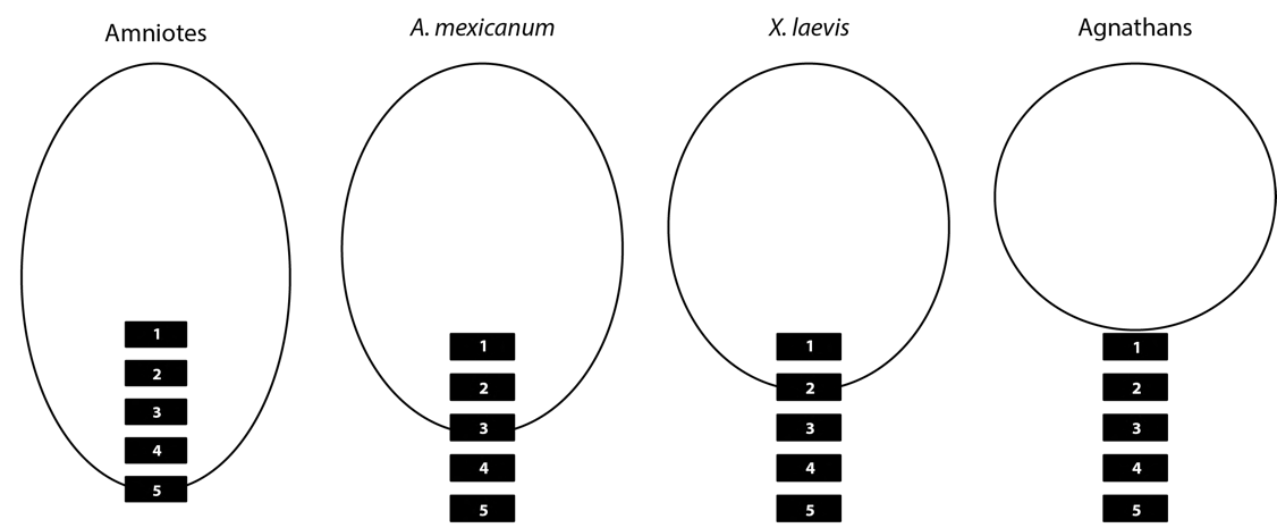

B
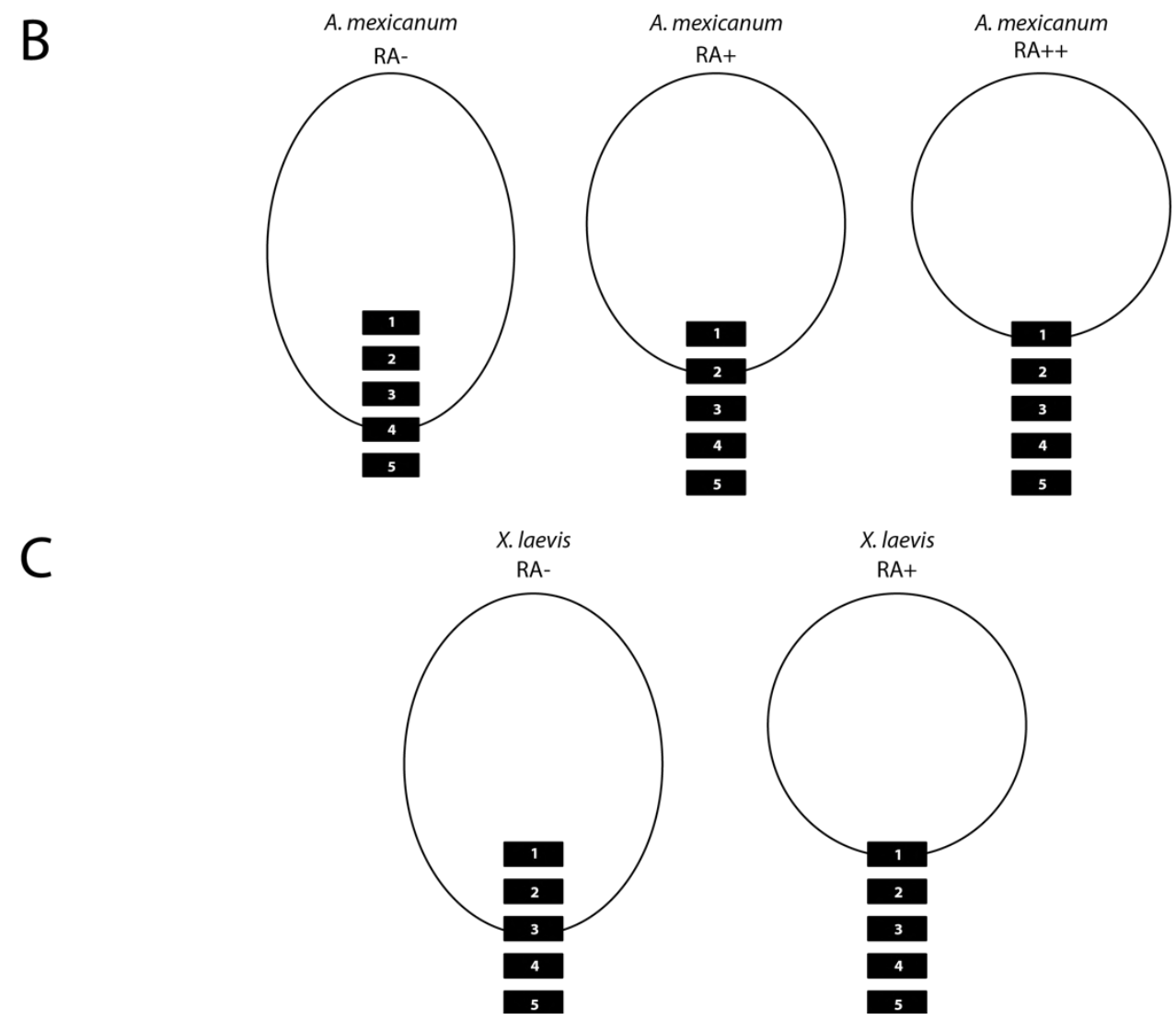

Figure 4.14. Schematic showing normal contributions to the occipital arch and the homeotic transformations caused in the present study. A) The normal contributions to the occipital arch are shown for amniotes (five), A. mexicanum (three), X. laevis (two), and agnathans (zero). B) When retinoic acid is inhibited (RA-), A. mexicanum contributes four somites to the occipital arch, which approaches the amniote condition of five. When exogenous retinoic acid at low concentrations $(\mathrm{RA}+)$ is administered, A. mexicanum contributes two somites to the occipital arch, which is the typical condition for $X$. laevis. When higher concentrations of exogenous retinoic acid are administered $(\mathrm{RA}++)$, one somite contributes to the occipital arch. C) When retinoic acid is inhibited in $X$. laevis, three somites contribute to the occipital arch, which is the typical condition found in $A$. mexicanum. When exogenous retinoic acid is applied, one half of a somite contributes to the occipital arch as the exoccipital arch still forms. 
In X. laevis, when exogenous retinoic acid was applied, the resulting morphology at the skull-neck boundary resembled the morphology observed in developing hagfish (Figure 4.14; Oisi et al., 2013; 2015), where a substantial gap between the occipital arch and the first neural arch of the vertebral column, or first myotome, is present during development. Hagfish, and other extant agnathans, do not contribute any somites to the occiput (de Beer, 1937; Oisi et al., 2013; 2015). The similar morphologies between developing hagfish and $X$. laevis specimens exposed to exogenous retinoic acid suggest that the experimental $X$. laevis specimens had all but half an occipital somite transformed to axial segments. As the occipital arch still formed, there must be at least half a somite contributing to the occiput. The citral treatments caused a single neural arch to become attached to the occiput (Figure 4.12). This suggests this somite has transformed fate from a vertebral to an occipital somite in X. laevis. As in A. mexicanum, all levels of citral doses only caused a single somite to transform fate (discussed below). With an additional occipital somite, $X$. laevis specimens treated with citral are interpreted as contributing three somites to the occiput, which is the same number as A. mexicanum under normal conditions (Figure 4.14).

While previous studies have shown that applying exogenous retinoic acid to amniotes and $X$. laevis causes Hox gene expression to shift anteriorly, these studies in $X$. laevis did not describe the possible homeotic transformations and resulting morphological consequences on the skeleton. Comparable Hox gene manipulation studies in $A$. mexicanum have not been previously conducted. Here, I show for the first time with morphological data that the application of exogenous retinoic acid and a retinoic acid inhibitor (citral) during somitogenesis and somite patterning can cause homeotic 
transformations of somites along the anterior-posterior axis. To confirm these homeotic transformations, I used two methods. The first was the relative position of the hypoglossal nerve complex with respect to somite-derived structures along the anterior-posterior axis. I predicted here that the position of the hypoglossal nerve complex and the associated somites, would appear to change relative to one another with the application of retinoic acid and a retinoic acid inhibitor.

Under normal developmental conditions, the hypoglossal nerve complex passes laterally from the axial skeleton between the occiput and the first vertebral elements in $A$. mexicanum (Figure 3.14; Norris, 1908; Norris, 1913; Herrick, 1948; Edwards, 1976). In experimental $A$. mexicanum specimens that were stained for nerves, the hypoglossal nerve passed laterally in the same location - i.e., between the occiput and the first vertebral element no matter the treatment (Figure 4.7). However, notably, these specimens did not possess the other morphological changes in the skeleton one would expect if homeotic transformations had taken place. They did not have extra vertebral elements when exposed to exogenous retinoic acid, nor did they have the first vertebral element attached to the occiput via cartilage when exposed to citral. They instead had very minor changes to anterior portions of the skull and to the shape of the skull (Figures $4.4 ; 4.5)$. It is therefore likely the $A$. mexicanum selected for the nerve staining procedures did not possess any of the homeotic transformations observed in other specimens, and this could be for a couple of reasons. The first is that $A$. mexicanum embryos exposed to exogenous retinoic acid and citral did not respond equally to the treatment. This could be the result of either imperfect mixing when the chemical was added to the embryos' water (both were poorly water soluble and had to be diluted in DMSO) or possible natural variation in sensitivity to the 
chemicals. Secondly, it was rare for A. mexicanum specimens to survive the exogenous retinoic acid or citral treatments at levels required to induce a change. Specimens that survived their treatments were less likely to have severe homeotic transformations and were therefore more likely to be able to function (i.e., for $X$. laevis the experimental specimens had complete heads unlike the external morphology observed in previous studies where specimens had microcephaly [Durston et al., 1989; Sive et al., 1990; Ruiz i Altaba and Jessel, 1991]) Additionally, younger specimens did not stain well with Sudan Black B nerve staining protocols. Thus, in order to have good, visible nerves, the specimens needed to be at developmental stages where they had ossification. The original protocols I adapted for use in this project (Filipski and Wilson, 1984; Northcutt et al., 2000) stained mature fish (Filipski and Wilson, 1984) and ten-week-old to adult $A$. mexicanum specimens (Northcutt et al., 2000). Ten-week-old $A$. mexicanum specimens are approximately 30 to 40 days after hatching, or stage 54 or older (Nye et al., 2003). My specimens that had the most skull deformities did not survive past stage 50, or approximately one week after hatching (Nye et al., 2003).

In control $X$. laevis specimens, branches of the hypoglossal nerve complex pass laterally through the axial skeleton in two locations in specimens examined here: between the first and second vertebral elements and between the second and third (Figure 3.14; Naumann and Olsson, 2018). As with A. mexicanum, X. laevis embryos exposed to exogenous retinoic acid did not respond well to Sudan Black B nerve staining. Again, this was likely because the embryos did not survive to an old enough developmental stage for Sudan Black B nerve staining to work. However, X. laevis specimens exposed to citral developed well to advanced developmental stages and were able to be nerve stained using 
the Sudan Black B protocols. In experimental citral specimens, the hypoglossal nerve complex passed laterally between the occipital arch and the first vertebral element. This is the same morphology observed in control A. mexicanum specimens. Notably, the citral specimens that were nerve stained also showed a morphology consistent with homeotic transformations. Using the position of the hypoglossal nerve complex, $X$. laevis citral experimental specimens are interpreted as possessing three occipital somites.

Overall, it appears that the hypoglossal nerve complex is a reliable indicator of homeotic transformations, if the embryos can survive past hatching stages, and if Sudan Black B staining protocols can be properly adjusted for juvenile specimens. In the future, however, it will be important to find better nerve staining methods to use the hypoglossal nerve complex to show homeotic transformations in younger A. mexicanum and $X$. laevis specimens. Previous research on juveniles (e.g., stage 43) where A. mexicanum specimens were exposed to retinoic acid and examined the resulting morphology of the lateral lines and the resulting anterior cranial nerve (i.e., olfactory, optic, and trigeminal nerves) morphologies used immunohistochemistry to visualize the nerves (Northcutt et al., 1994; Gibbs and Northcutt, 2004). In the future, I would be interested in attempting immunohistochemistry methods to study the resulting nerve morphology in young experimental specimens.

The second method used to confirm homeotic transformations was the GFPpositive somite grafting experiments conducted on $A$. mexicanum. With the baseline of contributions of somites two and three to the occiput determined in Chapter 3 and with their contributions to muscles previously published (Piekarski and Olsson, 2007), it was expected that if homeotic transformations were occurring as a result of the exogenous 
retinoic acid and citral treatments the fate of these somites would change. Specifically, it was predicted that with application of exogenous retinoic acid the fate of somite two would be transformed into the fate of somite three, and the fate of somite three transformed into the fate of somite four. With citral treatments, it was predicted that the fate of somite three would be transformed into the fate of somite two. The results of the GFP-grafting experiments confirmed these predictions (Figure 4.8; 4.9). When exogenous retinoic acid was applied to specimens where somite two was grafted, the resulting muscle fate resembled a normal somite three graft (Figure 4.8A). Then, when exogenous retinoic acid was applied to specimens where somite three was grafted, the resulting muscle fate resembled a normal somite four graft (Figure 4.8B). The sectioned specimens allowed the somitic contributions of somite three to the cartilages of the occiput and vertebral column to be studied. In a specimen exposed to exogenous retinoic acid, labelled cells from somite three were located completely outside of the skull, within structures of the now first and second vertebral elements. This is the fate of somite four in a normal embryo (Figure 4.9B). In a specimen exposed to citral, labelled cells from somite three were located within the anterior portion of the occipital arch. The posterior portion of the occipital arch was unlabelled. This is the fate of somite two in a normal embryo. With somite three positioned entirely in the skull, the skull-neck boundary would then correspond to being within somite four, which is consistent with the whole-mount cartilage-stained morphological data (Figure 4.14B). Overall, these GFP-somite grafting experiments confirmed that homeotic transformations took place in the treated A. mexicanum specimens. In the future, it will be important to collect complementary grafting data in X. laevis. 
A final way one could confirm the occurrence of homeotic transformations, and reveal the potential mechanistic basis, would be to examine Hox gene expression domains in treated and untreated specimens. The unique combination of Hox genes expressed in a somite gives that somite its fate - i.e., cervical, thoracic, caudal, etc. (Kessel and Gruss, 1990; Ramírez-Solis et al., 1993; Burke et al., 1995; Nowicki and Burke, 2000; Wellik and Capecchi, 2003). The anterior expression boundary of certain paralogous Hox groups mark transitions along the anterior-posterior axis (Figure 1.5; Burke et al., 1995). For example, the cervical to thoracic boundary is marked by the transition between Hox groups five and six (Burke et al., 1995). Data collected in amniotes (e.g., Gaunt et al., 1988; Godsave et al., 1994; Burke et al., 1995; Manzanares et al., 2001; Gaufo et al., 2003) have shown that Hox three paralogs (occipital region expression) and Hox four paralogs (anteriormost cervical region expression) are of special interest in the story of skull-neck boundary evolution (Mallo et al., 2010). Previously published expression data for candidate Hox genes hoxb3 and hoxd4 in amniotes show that these genes may be particularly useful here (e.g., Gaunt et al., 1988; Gaunt, 1994; Gaunt and Strachan, 1994; Godsave et al., 1994; 1998; Manzanares et al., 2001; Gaufo et al., 2003; Rastegar et al., 2004; Kobrossy et al., 2006). Hoxb3's normal anterior expression limit is within somite five in amniotes and it is also present in the hindbrain (Gaunt et al., 1988; Gaunt, 1994; Godsave et al., 1994; 1998; Manzanares et al., 2001; Yau et al., 2002; Gaufo et al., 2003) and hoxd4's normal anterior expression limit is at the anterior boundary of somite seven in chicken (Gaunt and Strachan, 1994; Burke et al., 1995) and between somites four and five in mice (Rastegar et al., 2004; Kobrossy et al., 2006) and like hoxb3, it is also present in the hindbrain (Morrison et al., 1997). 
While similar gene expression data is not available for A. mexicanum, it is published for X. laevis. In X. laevis, hoxb3 is expressed strongly in the hindbrain and is expressed much more weakly in the mesoderm than observed in amniotes such as mice (Godsave et al., 1994). Many of the Hox group b genes are expressed more weakly in the mesoderm of X. laevis than in that of amniotes, with the exception of hoxb4 (Godsave et al., 1994). This expression pattern is also true of hoxd4 in X. laevis in comparison to that of chick or mice (e.g., McNulty et al., 2005). In the future, it will be important to collect gene expression data for hoxb3 and hoxd4 for normal development in A. mexicanum and the gene expression data for perturbed development in both A. mexicanum and X. laevis. While published gene expression data on hoxb3 perturbed by retinoic acid exists for X. laevis (Godsave et al., 1998), that study focused on applying enough exogenous retinoic acid to remove structures anterior to the hindbrain and eventually also the hindbrain in young embryos. A future study on hoxb3 and hoxd4 gene expression in the mesoderm, comparing expression patterns of normal and perturbed development to the vast literature on amniote Hox gene expression, will be necessary to confirm with absolute certainty that homeotic transformations caused by exogenous retinoic acid and citral were in turn the result of changes to homologous Hox genes that determine the fate of skull-neck boundary structures, and thus its location.

Aside from compiling evidence of homeotic transformations, an interesting result of the citral experimental specimens was only one segment being consistently recruited into the skull. Even in X. laevis specimens, which tolerated citral treatments well at higher doses when A. mexicanum consistently had very low survivability, only one additional segment appeared to be recruited into the skull. I expected that, like the exogenous retinoic 
acid treatments, higher concentrations would have stronger morphological effects. Citral, the chemical used in the current study to lower the concentration of endogenous retinoic acid in the developing embryo works by inhibiting the alcohol dehydrogenase that oxidizes retinol to retinaldehyde as well as the aldehyde dehydrogenase that oxidizes retinaldehyde to retinoic acid (Schuh et al., 1993; Tanaka et al., 1996; Scadding et al., 1999). Retinoic acid is important for many tissues in the developing embryo, not just Hox gene expression and patterning in the somites (Durston, 2019; Ghyselinck and Duester, 2019; Nolte et al., 2019). It is also important for heart, gut, eye, kidney, and reproductive tract development, as well as forelimb development (Cartry et al., 2006; Ghyselinck and Duester, 2019; Nolte et al., 2019). Previous citral experiments in X. laevis showed that this chemical severely disrupts the development of the kidneys (Cartry et al., 2006). Finding a concentration of citral that both inhibited retinoic acid enough to cause homeotic transformations at the skull-neck boundary, without adversely affecting these other important systems so the embryo could survive, was difficult. This difficulty highlights the importance of transgenic animals or genetic knockdowns for their more targeted approach versus global chemical treatments, which affect the entire embryo. With more time, and more embryos, higher concentrations could be attempted for X. laevis, which survived citral treatment well. For A. mexicanum specimens, which rarely survived citral treatments, higher concentrations may not be possible without disrupting other embryonic functions too much and a targeted approach might need to be developed.

Another interesting result occurred when exogenous retinoic acid was applied to $X$. laevis. Even when the specimens resembled the hagfish morphology (Figure 4.11D; Oisi et al., 2013; 2015), with a gap between the first neural arch and the otic capsule, the 
exoccipitals still formed. It is therefore likely that a partial somite was still contributing to the occipital arch even though the morphology resembled the hagfish, where zero occipital somites are present. This likely possibility would need to be confirmed with somite fate mapping studies in experimental specimens. It is also interesting to me that amphibians have exoccipitals at all. In mouse and chicken, all five occipital somites contribute to the exoccipitals, as well as to the basioccipital (Huang et al., 2000; Couly et al., 1993). Both the basioccipital and the exoccipital elements contribute to the occipital condyles, which are important for the joint that forms with the vertebral column and for head movement. In species where the basioccipital is reduced or absent, the exoccipitals are larger elements (Figure 1.3). Lissamphibians do not have a basioccipital (see Chapter 2), and while celllineage tracing is not available for the first somite, in A. mexicanum somites two and three contribute to the exoccipitals (see Chapter 3). Since even X. laevis, which likely contributes fewer occipital somites to the skull than A. mexicanum (Chapter 3), forms exoccipital elements, it is likely that there is some sort of evolutionary pressure for the exoccipitals to form from the occipital somites, pressure that the basioccipital is not under. Early research into homology of structures along the anterior-posterior axis found that while the association of elements with segments along the anterior-posterior axis can change, important elements will always form anyway (Goodrich, 1913). This is why morphological elements like the shoulder and pelvic girdles, or the hypoglossal nerve complex, will form even if the somites they are associated with change (Goodrich, 1913; Burke et al., 1995). In amniotes exposed to exogenous retinoic acid, the basioccipital is often malformed, the exoccipitals less so, and never to the extent of the basioccipitals (e.g., Kessel et al., 1990). That the exoccipitals formed here in all surviving specimens, even in the presence of high 
concentrations of retinoic acid, indicates a stronger selective pressure for this element to form and be retained, pressure that does not encompass every occipital element.

Finally, of particular interest is what could be the driving force behind the reduced number of occipital somites in lissamphibians. Transgenic A. mexicanum that fluorescently report retinoic acid signalling in the developing embryo may help to explain why fewer occipital somites are present (Monaghan and Maden, 2012). During somitogenesis, retinoic acid is being synthesized in somites three through seven and in the pronephros (Monaghan and Maden, 2012). In A. mexicanum, the anterior limit of the pronephros is found at the level of somite four, whereas in X. laevis it is found at the level of somite three (Figure 3.1). In chick, the anterior limit of the pronephros is located more posteriorly, and is associated with somite eight (Grinstein et al., 2013) and in mice it is associated with somite seven (Cartry et al., 2006). This slightly more posteriorly located anterior limit of the pronephros in amniotes compared to lissamphibians lines up with the slightly more posterior limit of the Hox gene paralogous groups expressing at the skull -neck boundary. Previous research has shown that exogenous retinoic acid in X. laevis also expanded the region taken up by the pronephros (Cartry et al., 2006), in addition to resulting in fewer somites contributing to the occipital arch. Thus, it is likely that the fewer occipital somites and the more anteriorly located limit of the pronephros observed in A. mexicanum and $X$. laevis is the result of retinoic acid signalling at a more anterior location in these species. It is possible retinoic acid being synthesized in the pronephros, which is located more anteriorly in A. mexicanum and X. laevis than in amniotes, may be influencing the adjacent Hox gene expression domains, and the skull-neck boundary is moved as a side effect. Or, the converse may be true; more anteriorly located retinoic acid is required for an event 
concerning the skull-neck boundary and the pronephros is along for the ride. Finally, both structures may have been shifted anteriorly to accommodate another, more posteriorly located structure. What, exactly, is driving the more anteriorly positioned retinoic acid in lissamphibians still needs to be discovered.

\section{5: Conclusions}

In sum, this study represents the first-time homeotic transformations at the skullneck boundary were performed in lissamphibian model organisms where the resulting morphology of skull-neck boundary structures - i.e., the occiput and first vertebral elements - were studied. I demonstrated that homeotic transformations can indeed be induced via two methods of evaluation, the relative location of the hypoglossal nerve complex to skeletal structures and cell-lineage tracing via GFP-positive grafted somites. Interestingly, the resulting homeotic transformations generated phenocopies of skull-neck boundary morphologies seen in other tetrapod groups, which indicates a similar phenomenon may have important evolutionary implications. In the future, it will be important to confirm that homeotic transformations occurred in correspondence to modifications in Hox gene expression. However, given the vast literature available on this front, it is very likely to be the case. The cause of a shift in Hox gene expression domains remains an open question at this point. However, I speculate that the anterior shift in the skull-neck boundary may be the result of an anterior shift of other structures located along the anterior-posterior axis, such as the adjacent pronephros. Ultimately, the driving force behind the loss of the occipital somites in lissamphibians has yet to be discovered. Future research on the broad possibilities, such as the pronephros, or other yet unidentified 
possibilities (see Chapter 5), will be important to discover why exactly lissamphibians contribute fewer somites to the occipital arch. 


\section{Chapter 5: Discussion}

Vertebrate life today exhibits a great diversity of morphological forms. We are only beginning to understand the developmental mechanisms that gave rise to this morphological diversity. The fossil record reveals even greater diversity and captures key morphological transformations throughout evolutionary history. Integrative approaches combining developmental and palaeontological data have greatly improved our understanding of many of these transformations, such as the fin-to-limb transition. However, the goal of the present thesis research was to shed light on a poorly understood transition, that of changes in the location of the skull-neck boundary in tetrapods.

In an attempt to improve our understanding of the skull-neck evolution, I have focused on elucidating outstanding aspects of our understanding of the morphology and development of this region in lissamphibians and their close fossil relatives. This thesis had two hypotheses that I wished to test. First, that the occipital morphology in lissamphibians is secondarily reduced compared to their fossil relatives, and second, that the perturbing Hox gene expression domains could induce homeotic transformation of structures at the skull-neck boundary. To test these hypotheses, I explored aspects of the lissamphibian skull-neck boundary from phylogenetic, morphological, and developmental aspects in three data chapters. Here, I summarize my thesis results and how they add to a growing collection of data that help us better understand skull-neck boundary evolution in tetrapods. 


\section{1: The lissamphibian occipital morphology is secondarily derived}

As mentioned several times throughout this thesis, the reduced morphology of the living lissamphibian occiput in comparison to that of amniotes has been mentioned or described in detail by several early comparative morphologists (e.g., Platt, 1897; Goodrich, 1913; Augier, 1931; Hunter, 1935; de Beer, 1937). These descriptions led many researchers, focusing their comparative anatomy efforts on extant species, to hypothesize that the lissamphibian occipital morphology represents the ancestral condition for tetrapods (e.g., Figure 1.2; Augier, 1931; Couly et al., 1993). Other researchers, focusing on the fossil record that revealed close relatives of lissamphibian had a more robust occiput than observed in extant lissamphibians, proposed an alternative hypothesis that the reduced lissamphibian occipital morphology is secondarily derived, and that instead the condition found in extant amniotes is the ancestral condition for Tetrapoda (e.g., de Beer, 1937; Romer and Edinger, 1942; Clack and Milner, 2009). However, this alternative hypothesis remained untested with phylogenetic analyses to determine character evolution and ancestral character state reconstruction. Without such data, any hypotheses about the derived or ancestral nature of the lissamphibian occipital morphology remained speculative.

In Chapter 2, I showed, using comprehensive phylogenetic analyses and ancestral character state reconstructions, that the lissamphibian occipital morphology is indeed reduced compared to their fossil relatives. Furthermore, as with other morphological transitions (e.g., fin-to-limb transition), the stepwise reduction of elements in the lineage leading to crown Lissamphibia is captured in the fossil record. The gradual reduction of the braincase from a heavily ossified feature in distant lissamphibian relatives to less robust 
elements amongst Amphibamiformes, to the eventual loss of the basisphenoid and basioccipital elements completely is captured in the fossil record (Figure 2.4).

In Chapter 3, I then went on to describe the development of the lissamphibian skull, with an emphasis on the occiput, in two lissamphibian model organisms: Ambystoma mexicanum for salamanders and Xenopus laevis for frogs. Both species are widely used in developmental, genetic, and for A. mexicanum, limb regeneration studies (e.g., Maden et al., 1983; Gardiner et al., 1995; Yang et al., 1999; Kragl et al., 2009; Harland and Grainger, 2011; Godwin et al., 2013; Piekarski et al., 2014). However, these two species lacked indepth descriptions of skull development that documented both the nature of somitic contributions and normal skull development stage by stage, complete with variation within developmental stages. I applied cell-lineage tracing techniques for somitic contributions to the skull and integrated these data with the growth series and hypoglossal nerve complex data in both species. I was able to confirm the incorporation of three somites in the occiput of $A$. mexicanum and provide support for the incorporation of two somites in the occiput of $X$. laevis. The stage-by-stage developmental data of the skulls presented here are easily integrated into previously published, widely used staging tables based primarily on external morphology for $X$. laevis (Nieuwkoop and Faber, 1994) and limb morphology for $A$. mexicanum (Nye et al., 2003). Together these three data sets (somitic contributions, normal skull development, and hypoglossal nerve morphology) provide a baseline to which all other lissamphibians can be compared. Most importantly for this thesis, they provide a baseline to which I could compare the outcomes of perturbed development observed in my experimental specimens (Chapter 4). Furthermore, these baseline developmental growth series based on the skull have become incredibly important in recent years as many of the 
older, original descriptions of these model species did not focus on variation within developmental stages. Without a record of developmental variation, it becomes impossible to properly compare perturbed development to the range of normal development exhibited by a species. Finally, with these data in hand, I was able to accurately describe the morphological consequences of perturbed development and compare experimental animals to these normal developmental baselines in Chapter 4.

\section{2: The reduced lissamphibian occipital morphology likely arose as the result of shifting Hox gene expression domains}

Changes in Hox gene expression domains along the anterior-posterior axis have occurred at key morphological transformations (e.g., the snake bauplan or the specialized regionalization of mammal vertebrae compared to basal amniotes) across evolutionary time, as shown in studies on Hox gene expression differences in extant vertebrates (Montavon and Soshnikova, 2014; Jones et al., 2018; Woltering et al., 2019). Hox gene knockdown or changes in expression experiments have demonstrated for decades that changes in Hox gene expression at the occiput causes changes in structures at the skullneck boundary (e.g., Durston et al., 1989; Kessel et al., 1990; Ruiz i Altaba and Jessel, 1991; Conlan and Rossant, 1992; Ramírez-Solis et al., 1993; Manley and Capecchi, 1997; McNulty et al., 2005; Kobrossy et al., 2006). Retinoic acid, a chemical that is synthesized in early development within the embryo and is incredibly important for a variety of developmental systems, is also heavily involved in the regulation of Hox gene expression, especially the paralogous groups one through four, which are expressed within the head and near the skull-neck boundary (Durston, 2019; Ghyselinck and Duester, 2019; Nolte et al., 2019). Numerous experiments have shown that changing the expression domains of Hox genes either via applying exogenous or inhibiting retinoic acid, or by knocking down 
specific Hox genes, produces segmental or homeotic transformations along the anteriorposterior axis of elements derived from paraxial mesoderm, like the somites (e.g., Kessel et al., 1990; Conlan and Rossant, 1992; Ramírez-Solis et al., 1993).

In Chapter 4, I described homeotic transformations at the skull-neck boundary that occurred in A. mexicanum and $X$. laevis when embryos during or immediately before somitogenesis were exposed to exogenous retinoic acid, or a retinoic acid inhibitor (citral). I provided evidence that homeotic transformations of skull-neck boundary structures could be induced in these species using two datasets: first with the relative position of the hypoglossal nerve complex to skeletal structures and second with GFP-positive labelled somite cell-lineage tracing. Both of these datasets confirm that homeotic transformations occurred, wherein the relative location of the hypoglossal nerve seemingly changed, and GFP-positive somites changed fate, when embryos were exposed to retinoic acid or a retinoic acid inhibitor (although only the nerve data is present for X. laevis and GFPpositive labelled lineage tracing for A. mexicanum). In the future, it will be important to improve nerve staining methods and expand GFP-labelled cell lineage tracing studies in both model organisms. Together, these data, along with Hox gene expression data for the skull-neck boundary will be invaluable for comparing gene expression and skull-neck boundary data with amniotes.

Homeotic transformations, the change in the morphology of one segment to that of another segment, are well understood and widely demonstrated in the postcranial axial column. Here, I showed that homeotic transformations likely underlie the origin of the diversity of occipital composition in tetrapods, and potentially vertebrates as a whole. This is supported by the morphologies seen among experimental animals produced here, where 
exposure of A. mexicanum and X. laevis to retinoic acid produced phenocopies of frogs and agnathans, respectively. Additionally, when exposed to the retinoic acid inhibitor citral, $A$. mexicanum and X. laevis developed as phenocopies approaching the amniote condition and salamanders, respectively. When combined with a review of the fossil record (Maddin et al., 2020), it is revealed that an amniote-like condition of occipital composition was fixed at the base of Tetrapoda, wherein the anteriormost somites up to the level of the posteriormost root of the hypoglossal nerve (typically comprising five occipital somites). This pattern has been maintained in virtually all tetrapod taxa, with the exception of lissamphibians. Future research into determining how and why these homeotic transformations took place will be invaluable for our understanding of the development and evolution of the skull, and clues may come from different anatomical systems (i.e., the pronephros).

\section{3: Heterochrony versus heterotopy}

For lissamphibians, heterochronic processes leading to paedomorphosis have long been suggested to have been important drivers in the origin of their unique form (e.g., Bolt, 1969; 1977; Milner, 1988, Boy and Sues, 2000). Heterochrony is an evolutionary change in developmental timing or rate (Zelditch and Fink, 1996). It has been suggested that heterochrony played an important role in the evolution and diversification of lissamphibians (Alberch, 1989; Sheil et al., 2014). For example, the palatal morphology, cheek bones, and skull roof of adult lissamphibians all resemble the juvenile morphology in close lissamphibian fossil relatives in Amphibamiformes, which is consistent with truncated development (Schoch, 2014a). Some skull elements that fail to form in lissamphibians but are present in close fossil relatives are later ossifying elements in these 
fossil relatives, such as the postfrontal, jugal, or tabular (Schoch, 2014a). In a careful analysis, Schoch (2013a) demonstrated the extent to which similar morphologies could be produced when widely separated lineages undergo similar heterochronic processes, and additionally when these lineages undergo miniaturization. Development in some lissamphibians and in their closest relatives is truncated for certain, as many branchiosaurids and extant salamanders are neotenic (a form of somatic truncation; Schoch, 2009). However, the changes in composition of the occiput identified here, and the underlying developmental basis, are not consistent with heterochrony.

That the loss events noted here in the lissamphibian braincase do not seem to be homoplastic with most other Paleozoic tetrapods that underwent similar heterochronic processes, such as miniaturization (Fröbisch and Schoch, 2009; Schoch, 2013a), conflicts with the idea of heterochrony as a source of the reduced lissamphibian occiput and speaks to the resilience of the braincase against such developmental perturbations. Instead, the loss of elements in the occiput appears to be more likely the result in spatial changes in development. An evolutionary change of spatial patterning and positioning of developmental processes is known as heterotopy (Zelditch and Fink, 1996). Changes to occipital elements can be induced in amniotes by changing the spatial pattern of Hox gene expression (Durston et al., 1989; Kessel et al., 1990; Ruiz i Altaba and Jessel, 1991; Conlan and Rossant, 1992; Ramírez-Solis et al., 1993; Manley and Capecchi, 1997; McNulty et al., 2005; Kobrossy et al., 2006). Here, I demonstrated in lissamphibians that exposing developing embryos to exogenous retinoic acid or a retinoic acid inhibitor caused homeotic transformations that generated phenocopies of skull-neck boundary morphologies seen in other tetrapod groups (Figure 4.14). Changing the spatial patterning of Hox genes, and thus 
heterotopy, is therefore required to produce the unique lissamphibian occipital morphology.

For the occiput, a unique heterotopic event likely gave rise to the unique, derived, lissamphibian morphology and that of their closest, Temnospondyl relatives. While lepospondyls, another Palaeozoic amphibian lineage that some authors propose may be the close relatives of some or all lissamphibians (Figure 2.1; Laurin and Reisz, 1997; Vallin and Laurin, 2004; Marjanović and Laurin, 2013) share a number of dermal bone losses with lissamphibians (Marjanović and Laurin, 2015), the dermal region of the skull is under less selective pressure than the braincase (Cardini and Elton, 2008; Goswami and Polly, 2010; Maddin et al., 2012b; Brazeau and de Winter, 2015; Maddin, 2015). These losses are unique to extant lissamphibians, and their gradual loss can be observed in their closest fossil relatives. These losses are consistent with both a Temnospondyl origin for lissamphibians, and with heterotopy as the driver.

\section{4: What drove the anterior Hox gene shift?}

As I mentioned in Chapter 4, the source of shifting Hox gene boundaries and reduction at the occiput is currently unknown. Either evolutionary pressure to modify the skull-neck boundary arose at some point, or alternatively, changes in the location of the skull-neck boundary may be a side effect of morphological changes in other parts of the embryo. Data in support of the former are currently lacking despite the fossil evidence indicating a stepwise reduction of occipital elements occurred in the lineage leading to extant lissamphibians (Figure 2.4); however, data from other parts of the embryo may hold some promising clues. In Chapter 4, I noted the complementary location of the pronephros and how in both $X$. laevis and A. mexicanum, this structure is positioned more anteriorly 
than in amniotes (Figure 3.1; Cartry et al., 2006; Monaghan and Maden, 2012; Grinstein et al., 2013). When exogenous retinoic acid was applied to $X$. laevis specimens, it not only causes a reduction in the somitic contributions to the occiput (Figure 4.10; 4.13), but it also causes an expansion of the region taken up by the pronephros (Cartry et al., 2006). However, the position of the pronephros is not the only possible factor that may be driving this anterior shift in Hox gene expression in lissamphibians.

A second possible driver of the unique lissamphibian morphology at the occiput is the position of the shoulder girdle. In chick, the shoulder girdle's anterior limit is associated with the sixteenth somite, whereas in mice it is associated with the ninth (Burke et al., 1995). In lissamphibians, the shoulder girdle is positioned much more anteriorly. It is associated with the second somite in X. laevis (Burke et al., 1995), and I have noted that in A. mexicanum it is associated with the fourth somite. Hoxc6 is a marker for the cervical to thoracic transition and it is also located more anteriorly in $X$. laevis than in chick or mouse (Burke et al., 1995). In X. laevis, hoxc6 has an anterior expression limit at the fourth somite, but its anterior limit is at the twelfth somite for mice and the nineteenth for chick. It is possible that the anterior shift of the shoulder girdle in lissamphibians secondarily caused a reduction in the number of somites that contribute to the occiput, or perhaps another morphological transition has caused an anterior shift in both regions. Ultimately, Hox gene expression data for these anterior somites in a variety of lissamphibian model organisms, not just in $X$. laevis, will be important to our understanding of how heterotopy has shifted gene expression in lissamphibians compared to amniotes.

FGF signalling is another possible candidate for the anterior shift in Hox genes. In Chapter 4, I described research that identified an opposing gradient between retinoic acid 
and FGF/wnt is key for the formation of somites during somitogenesis (Figure 4.1; Gomez and Pourquié, 2009). However, like retinoic acid, FGF signalling pathways are important for a variety of functions in the developing embryo. FGF is important for neural and tail development, as well as paraxial mesoderm formation (Fletcher and Harland, 2008). As with exogenous retinoic acid, overexpression of $F G F 8$ posteriorizes segments and can prevent head formation in $X$. laevis via inhibition of the forebrain and midbrain genes (Christen and Slack, 1997; Fletcher et al., 2006). Loss of function research has shown that FGF8 suppresses Hox genes in the first rhombomere, thus defining the anterior limit of Hox genes (Irving and Mason, 2000). In the anterior hindbrain, FGF8 signalling is dominant over the retinoic acid signal and this dominant signal provides a Hox gene free region for the cerebellum to form (Irving and Mason, 2000). Posterior to the first rhombomere, the retinoic acid anterior-posterior gradient that is important during somitogenesis also activates the individual Hox genes in the hindbrain, with each Hox gene activated by a different concentration of retinoic acid (Irving and Mason, 2000; Begemann and Meyer, 2001). It is therefore possible that changes in FGF8 signalling during lissamphibian evolution may have reduced the size of the region in which these anterior, occipital bone forming Hox genes express. To test this theory, careful comparison of $F G F 8$ signalling in amphibians and amniotes in the anterior region of the embryo would be necessary.

\section{5: Future directions}

My $\mathrm{PhD}$ research opens up several potential avenues of study. In addition to the potential future experiments described above, I cannot stress enough the importance of gene expression data. In the future, Hox gene data, and other important genes expressed in 
the anterior region during development, will be invaluable. While gene expression data is published for X. laevis, these studies focused on Hox gene expression in the hindbrain, and therefore published expression data in very young embryos. Anterior Hox gene expression data has not yet been published for $A$. mexicanum. I would be interested in comparing Hox gene expression data in older embryos, with comparable stages in amniotes.

Secondly, at present nerve staining protocols on juvenile amphibians are problematic. Immunohistochemistry is one solution to the issue with nerve staining protocols with Sudan Black B. I would be particularly interested in using immunohistochemistry to confirm homeotic transformations in my experimental specimens. Also, using GFP-labelled somite lineage tracing to confirm homeotic transformations in X. laevis would be helpful here. While I had hoped to confirm the somitic contributions to the occipital arch in X. laevis, time constraints and difficulty with getting embryos to survive after somite labelling prevented this dataset from being accomplished here. In the future, confirming the number of somites that contribute to the occiput in X. laevis will be invaluable to our understanding of the occipital composition in frogs.

While I have shown here that changes in Hox gene expression domains may have led to the reduced lissamphibian occiput, it has yet to be shown that Hox gene expression domains shifted during lissamphibian evolution. In order to determine if such a shift happened, the fossil record is important. In extant vertebrates, morphological transitions along the anterior-posterior axis are marked by the anterior limit of certain Hox gene paralogous groups (Figure 1.5; Burke et al., 1995). While many fossil Amphibamiformes consist of only a skull, many branchiosaurids and less closely related temnospondyls have 
post-cranial material (e.g., Schoch and Milner, 2014). Comparing the location of the shoulder girdle in fossil temnospondyls with extant lissamphibians and amniotes may reveal a gradual, or a sudden, shift in Hox gene expression, revealed by the position of the shoulder girdle along the anterior-posterior axis in fossil amphibians.

From a developmental perspective is the potential to continue to test the hypothesis presented here that heterotopy is driving the lissamphibian occipital morphology. Length of time of developmental processes is easily perturbed in lissamphibians. Frog metamorphosis can be induced early by decreasing water level in their tanks, thus truncating their development (Merilä et al., 2000), whereas A. mexicanum, a paedomorphic species, can be induced to undergo metamorphosis and thus a longer period of development via exogenous thyroid hormone (Smirnov and Vassilieva, 2005). In order to be certain that the lissamphibian morphology is the result of heterotopy and not heterochrony, development should be truncated in $X$. laevis, and expanded in A. mexicanum and the resulting skull morphology should be studied. If similar experimental morphologies observed when perturbing Hox gene expression domains cannot be replicated in experiments that either prolong or truncate lissamphibian development, then it is likely the lissamphibian occiput is the result of heterotopy.

The second avenue of developmental, extant research this $\mathrm{PhD}$ thesis opens up is the underlying developmental mechanisms that could be driving the reduced lissamphibian occipital morphology. In Chapter 4, I described how the reduced occipital morphology may be the result of an anterior shift in the location of the pronephros. Here, I described how it may also be the result of the position of the shoulder girdle, which is located more anteriorly in lissamphibians than in amniotes (Burke et al., 1995). I also suggested that the 
$F G F 8$ signalling pathway may be the culprit, as overexpression of $F G F 8$ can prevent head formation in X. laevis (Christen and Slack, 1997; Fletcher et al., 2006). The first step I would take to explore these potential upstream causes is to produce a somitic fate map for all somites present in A. mexicanum and X. laevis via GFP-positive somite grafting. Such fate maps exist for chick and mouse, and the fate of each somite is known in these amniote model organisms (Burke et al., 1995). Only the fate of some somites is known for $A$. mexicanum (Piekarski and Olsson, 2007; Piekarski, 2009; Piekarski and Olsson, 2014) and X. laevis (Burke et al., 1995). Knowing the fate of each somite in lissamphibian model organisms will allow them to be more easily compared to somite fate in amniote model organisms, to check for differences more posterior to the occipital arch or the shoulder girdle. Secondly, while transgenic A. mexicanum allowed the location of synthesis of retinoic acid to be described (Monaghan and Maden, 2012), comparative research has not been conducted in $X$. laevis. The use of transgenic $X$. laevis to visualize retinoic acid synthesis will be useful to determine if, like the position of the pronephros, retinoic acid is being synthesized in a more anterior location than observed in A. mexicanum. Finally, while $F G F 8$ gene expression has been analysed in developing and regenerating $A$. mexicanum limbs, it has not been studied in the head. Whole embryo $F G F 8$ expression has been studied in X. laevis (Hayashi et al., 2004). Generating $F G F 8$ gene expression data for A. mexicanum and then making comparisons with $X$. laevis and amniotes will be important to determine if differences in expression may have caused the anterior shift in the skullneck boundary in lissamphibians.

\section{6: Conclusions}


The goal of this $\mathrm{PhD}$ thesis was to contribute to and enhance our understanding of the morphology and development of the skull-neck boundary in lissamphibians and their close fossil relatives. With my work here, I have shown that the occipital morphology in lissamphibians is secondarily reduced compared to their fossil relatives. Secondly, via my manipulation of Hox gene expression domains and the homeotic transformations my manipulations caused at the occiput, I have shown that the reduced morphology observed in lissamphibians was likely driven developmentally by changes in Hox gene expression domains. This research contributes to our understanding of the evolution of the unique amphibian occipital morphology. My integrative approach has helped to elucidate the relationship between morphological transformations and the developmental processes underlying them. This work has also shown, for the first time, the role of heterotopy in amphibian evolution. 


\section{Appendices}

\section{Appendix A: Character list for Chapter 2}

The following character list was modified from Schoch (2018).

1. Laterally exposed palatine (LEP). Palatine overplated by jugal and lacrimal with no lateral exposure (0); palatine wedging between lacrimal and jugal to make contribution to skull roof and orbital margin (1); maxilla contributes to orbital margin by separating jugal and lacrimal in the absence of lateral exposure of palatine (2) (based on Sumida et al., 1998; Schoch and Rubidge, 2005); lateral exposure of palatine present and excluded from orbital margin by jugal and lacrimal contact (3). An LEP was shown by Boy (1995) to be present in adults of Micromelerpeton credneri, and it is also likely to exist in adult branchiosaurids (Apateon gracilis), but coded "?" because of disarticulation.

2. Dorsal quadrate process (DQP). Quadrate having smooth posterodorsal side (0); quadrate with prominent dorsoposterior outgrowth, the quadrate process (1). With the exception of larval-neotenic taxa (Branchiosauridae, Micromelerpetidae), the derived condition has been reported from all dissorophoids (Bolt, 1969; Schoch and Rubidge, 2005).

3. Vomerine depression. Ventral surface of vomers flat and element divided into anterior and posterior portion by transverse ridges that may or may not bear transverse tooth row (0); single unpaired depression in anterior portion of vomers that may or may not house an opening (1) (Schoch and Rubidge, 2005).

4. Parasphenoid dentition. Basal plate of parasphenoid bearing shagreen of small teeth (denticles) anteromedially (0); plate entirely smooth (1) (Schoch and Rubidge, 2005). In the Dissorophoidea, the derived condition is reported from two different families, the Branchiosauridae (Boy, 1972, 1987) and Dissorophidae (Berman et al., 1985).

5. Parasphenoid denticle field. Well established, with triangular outline and with apex reaching onto base of cultriform process $(0)$; denticle field greatly expanded anteriorly to cover most of the cultriform process (1) (Schoch and Rubidge, 2005). Within Dissorophoidea, the derived condition is only known in some amphibamids (Micropholis, Pasawioops, Amphibamus, Platyrhinops) and Eocaecilia.

6. Parasphenoid basal plate. Roughly quadrangular dimensions, as long as wide (0); basal plate much shorter than wide, reaching about half the width (1) (Schoch and Rubidge, 2005). 
7. Vomerine denticle field. Vomer covered with a more or less dense shagreen of teeth in addition to obligatory fang pair (0); shagreen confined to juvenile stages and/or absent throughout ontogeny (1) (Schoch and Rubidge, 2005).

8. Vomerine fangs. Vomer lacking fangs in its medial portion, outside lateral tooth arcade, but having smaller accessory teeth in that region (0); vomer with additional fang pairs posterior to midvomerine depression (1) (Schoch and Rubidge, 2005).

9. Pterygoid-vomer contact. Suture between pterygoid (palatine ramus) and vomer (0); pterygoid contacting only palatine (or ectopterygoid) and lacking suture with vomer (1) (Schoch and Rubidge, 2005).

10. Pterygoid flange. Palatine ramus of pterygoid merging continuously into basipterygoid ramus (0); palatine ramus broadening abruptly to form a pronounced transverse flange, giving the lateral margin a rectangular shape (1). The newlydefined apomorphic state is only present in dissorophids; in trematopids and amphibamids, the flange is rounded (Schoch and Rubidge, 2005).

11. Palatine, ectopterygoid. Palatine and ectopterygoid much wider than maxilla (0); palatine and ectopterygoid reduced to narrow struts not wider than adjoining maxilla (1). The two bones are here considered together, because their width evolved in parallel and was probably linked. (Schoch and Rubidge, 2005).

12. Interpterygoid vacuity. Roundish or oval in outline (0); greatly expanded laterally at mid-level (1) (Milner, 1993).

13. Narial flange. Ventral (inner) side of prefrontal, lacrimal, and nasal smooth (0); inner side of these bones forming complicated bar-like structure (narial flange), permitting contact with antorbital bar (1) (Boy, 1981).

14. Prefrontal process. Prefrontal forming simple suture with lacrimal laterally (0); prefrontal underplating lacrimal widely by means of ventral prefrontal process contacting palatine (1) (Boy, 1981; Werneburg, 1989).

15. Tabular size. Tabular narrower than postparietal, but reaching almost the same size as latter (0); tabular minute and laterally constricted by unique enlargement of otic notch (1) (Schoch and Rubidge, 2005).

16. Tabular-squamosal contact. Tabular and squamosal widely separated by supratemporal (0); squamosal meeting tabular, excluding supratemporal from otic notch (1) (Yates and Warren, 2000; Schoch and Rubidge, 2005).

17. Postparietal length. Postparietal forming transversely rectangular or quadrangular element (0); postparietal abbreviated and reduced to narrow, poorly ornamented strut at posterior margin of skull table (1) (Schoch and Rubidge, 2005). 
18. Squamosal-supratemporal suture. Nearly as long as supratemporal itself (0); or shorter, reaching only one third or less of length of supratemporal (1) (Schoch and Rubidge, 2005).

19. Supratympanic flange (=semilunar flange in Schoch and Rubidge, 2005, terminology following Bolt, 1974a). Squamosal continuously ornamented around margin of otic notch (0); squamosal having dorsally exposed and ornamented area (supratympanic flange) stepping abruptly into steeply aligned, poorly ornamented portion (1).

20. Semilunar flange (=supratemporal flange of Schoch and Rubidge, 2005). Supratemporal without ventral projection into otic notch (0); supratemporal forming marked ventral flange participating in medial bordering of otic notch (1).

21. Prefrontal-postfrontal. Firmly sutured on the dorsal side, excluding the frontal from the orbital margin (0); both elements separated by frontal, at least dorsally (1). Whereas this character varies in some capitosaurs (Schoch, 2000), it has not been found to do so in dissorophoids. Notably, the stratigraphically earliest micromelerpetontids (Limnogyrinus elegans), branchiosaurids (Branchiosaurus salamandroides) and amphibamids (Amphibamus grandiceps, Platyrhinops lyelli) have the plesiomorphic condition, whereas this is not known from any trematopid or dissorophid.

22. Interorbital width. Narrow to moderately wide interorbital region in the $0.2-0.24$ range (interorbital width/skull length) (0); or substantially wider (0.27-0.33) (1). In the outgroups, Dendrysekos has an unusually wide interorbital region, which is why Sclerocephalus has been taken as a guide to the primitive condition (Modified from Anderson et al., 2008b).

23. Palpebral ossifications. No ossifications other than sclerotic ring (0); numerous palpebral ossicles at medial margin of sclerotic ring (1). This character, first documented by Credner (1881, as "Skleralpflaster") in Branchierpeton and described in detail by Boy (1972) in Micromelerpeton, was later emphasized by Daly (1994) as typical of amphibamids. However, it is not confined to Micromelerpetidae and Amphibamidae, but also known from Broiliellus brevis (Schoch 2012) and probably widespread among dissorophoids (Holmes et al., 2013). Palpebral ossicles are not to be confused with dentigerous platelets which cover the interpterygoid vacuities of many temnospondyls, a character that was much more widespread and is not considered here.

24. Stapes. Stapes with pronounced dorsodistal curvature directed towards dorsally located otic notch (0); or abbreviated without dorsodistal curvature, directed mostly laterally towards vertically aligned otic notch (1). The derived character-state is present in branchiosaurids, amphibamids, trematopids, and dissorophids (Schoch, 2012; Schoch and Anderson, 2016). 
25. Prefrontal-jugal contact. Absent (0); present (1). The derived state is found in Sclerocephalus (representing the vast stereospondylomorph clade and eryopids that all share this feature). The resulting pattern in which the lacrimal does not border the orbit evolved convergently in Cacops morrisi (Reisz et al., 2009), where it results from an anterior expansion of the LEP, unlike in Sclerocephalus where the jugal extends further anteriorly.

26. Maxilla dentition. Extending posterior to the level of the posterior margin of the orbit (0); terminating at the level of such margin or anterior to it (1). This is an exclusive dissorophid character, which is best seen in Cacops (Schoch, 2012).

27. Skull outline. Absence (0) or presence (1) of inward inflection of skull outline in dorsal view at the level of the maxilla-premaxilla suture (Schoch and Rubidge, 2005).

28. Septomaxilla. At the posterior rim of naris (0); at mid-level of naris, pointing inside (1). The derived state is present in all trematopids and dissorophids, differing from all other temnospondyl groups (Schoch, 2018).

29. Parietal width. More (0) or less (1) than two and a half times as long as wide (Schoch and Rubidge, 2005).

30. Postparietal length. Postparietals less (0) or more than (1) four times wider than long (Schoch and Rubidge, 2005).

31. Postorbital. Narrowing to an acute posterior point (0); not narrowing, ending blunt (1).

32. Vomer (posterior projection). Absent (0) or present (1) (Schoch, 2018).

33. Vomer (tooth row). Vomer with (0) or without (1) a toothed, raised crest running anteroposteriorly and lying mesial to the choana (Schoch and Rubidge, 2005).

34. Palatine and interpterygoid vacuity. Palatine partially or fully excluded from vacuity by pterygoid (0); bordering vacuity along its entire medial margin (1) (Reformulated from Milner, 1990; Yates and Warren, 2000).

35. Cultriform process. Moderately wide and flat on ventral side (0); throughout thin and round in cross-section (1) (Schoch, 2012).

36. Exoccipital-tabular contact. Absent (0); present (1). The derived state is a synapomorphy of eryopiforms (Sclerocephalus) and also reported in zatracheids (Boy, 1990). 
37. Exoccipital-postparietal contact. Present (0); absent (1) (Coding reverted from Polley and Reisz, 2011), because absence is clearly exclusive to cacopines in the present set of taxa).

38. Position of jaw articulation. Posterior to (0), level with (1), or anterior to (2) the posterior facets of the exoccipitals (Yates and Warren, 2000).

39. External narial opening. Uniform, oval shaped margin (0); posteriorly expanded at the expense of lacrimal, with distinct anterior and posterior regions giving external naris an overall 'key-hole' shape (1) (Dilkes, 1990).

40. Internarial fenestra. Absent (0); present (1) (Dilkes, 1990; Anderson et al., 2008b).

41. Marginal teeth of upper jaw. Uniform in size (0); caniniform teeth on premaxilla and maxilla (1) (Dilkes 1990).

42. Prearticular. Inflection of the prearticular along the medial rim of the adductor fossa: absent (0); present (1) (Dilkes, 1990).

43. Ventral border of otic notch. Slopes posteroventrally (0); nearly horizontal (1) (Dilkes and Reisz, 1987). Within the Trematopidae, this character may have been subject to ontogenetic change or scaling, as small trematopids have the plesiomorphic, large ones the apomorphic condition.

44. Median vomerine septum. Absent (0); present (1) (Dilkes and Reisz, 1987; Dilkes, 1990).

45. Tabular process. Any state between short and absent (0); curves gradually to meet the robust quadrate process (1); bent down sharply at approximately a right angle to the dorsal edge of the skull table and fused to the quadrate process (2) (Dilkes and Reisz, 1987).

46. Stapedial foramen. Present (0); absent (1) (Boy, 1981; Daly, 1994). This character was long believed to separate trematopoids from dissorophids, with the latter considered to lack a foramen, However, Cacops morrisi clearly has a stapedial foramen (Gee and Reisz, 2018).

47. Knobby exostoses (skull roof). Absent (0); present (1) (Daly 1994). The derived state is found in dissorophids, some of which have excessive bone growth in the skull (DeMar, 1968).

48. Subnarial lacrimal process. Long (0); short (1) (Sumida et al., 1998).

49. Squamosal. Semilunar curvature along the ventral border of the supratympanic flange: absent (0); present (1) (Berman et al., 1985; Dilkes, 1990). 
50. Dorsal rim of occiput. Gently ornamented (0); ornament forming marked transverse ridge (1) (Schoch, 2012).

51. Ratio of preorbital length to postorbital length. Preorbital length greater than postorbital length by more than $10 \%(0)$; preorbital and postorbital lengths approximately equal (1); postorbital length greater than preorbital length by greater than $10 \%(2)$ (Yates and Warren, 2000).

52. Suborbital bar height. Greater than $10 \%$ of the total midline skull length ( 0$)$; less than $10 \%$ of the total midline skull length (1) (Yates and Warren, 2000).

53. Minimum distance otic notch-posterior orbital margin. Greater than $25 \%$ of the total midline skull length (0); between 10 and $25 \%$ of the total midline skull length (1); less than $10 \%$ of the total midline skull length (2) (Schoch, 2012).

54. Basipterygoid articulation. Discrete facet for ball-and-socket joint or overlap (0); firmly sutured at mid-level of widened basipterygoid process (1) (Yates and Warren, 2000).

55. Postorbital-supratemporal. Sutured (0); separated by postfrontal (1). This character appears to be polymorphic in Phonerpeton, but obligate in Cacops morrisi; it is unknown in the heavily ornamented taxa (Cacops aspidephorus, Kamacops, Zygosaurus).

56. Intertemporal. Present (0); absent (1). Although a clear-cut character, the intertemporal was lost several times independently in basal temnospondyls. In Sclerocephalus and Micromelerpeton, it has been reported as a polymorphism in several samples (Boy, 1972, 1988).

57. Osteoderms. Median unpaired osteoderms (one per vertebra) absent (0); or present (1) (Dilkes and Brown, 2007). DeMar (1966), who first studied the dissorophid armor in depth, distinguished two major types (see character 58), arguing for their independent evolutionary origin. However, there is no reason to conclude that the inner series of osteoderms is not homologous throughout dissorophids, just because its mode of articulation differs in sub-clades.

58. Osteoderm width. Narrow (0), wide (1). Dissorophus and Broiliellus texensis share wide (internal and external) osteoderms reaching at least the width of the two postparietals. In Aspidosaurus (sensu stricto) and Cacops, the osteoderms are much narrower, not exceeding the maximum width of the transverse processes (DeMar, 1966).

59. Cranial ridges. Dorsal surface of skull roof flush or with gentle ridges on prefrontal, orbital margin, and in the temporal region (0); or with pronounced ridges especially in the snout (1). Although Cacops and Kamacops, Scapanops, and 
Conjunctio also have these ridges, they are always shallower than in Dissorophus and the three Broiliellus species (Schoch, 2012).

60. Ilium (dorsal process). High and slender, distally not wider than shaft (0); or short and stout, not higher than base is wide, with broadened dorsal end (1). This is one of the more convincing characters shared by trematopids and dissorophids, as it never occurs in other dissorophoids (Daly, 1994; Schoch and Rubidge, 2005)

61. Parasphenoid plate. Lateral margin with or without moderate posterolateral projection (0); posterolateral wing expanded well beyond the level of the basipterygoid suture. Lateral projections of the basal plate are found in most amphibamids, branchiosaurids, and dissorophids, but they are most pronounced in Broiliellus and Dissorophus. The presence of a projection in state 0 is subject to ontogenetic changes and variation (Schoch, 2012).

62. Carotid artery. Exit foramina for carotid artery located on the parasphenoid plate (0); or at the base of the cultriform process (1). The derived state is clearly present in Cacops morrisi and Kamacops, but unknown in other cacopines; it may thus have been more widespread within this group. Dissorophus, Broiliellus, Aspidosaurus, Platyhystrix, and Conjunctio show the plesiomorphic state (Shishkin, 1968).

63. Tabular horn: posterior extension. Round or pointed but short (0); or elongated, forming the main portion of tabular (1). Within dissorophoids, the derived state is confined to the three Broiliellus species (Schoch, 2012).

64. Jugal. Dorsal surface of jugal regularly ornamented (0); or with large eminence bearing knobby ornament (1). This feature is most pronounced in Dissorophus, but also present in Broiliellus brevis (Schoch, 2012). It is unknown whether the derived state was age- or size-related, but it is certainly absent in Cacops and Kamacops.

65. Parasphenoid: muscle scars. The posterior and posterolateral parts of the parasphenoid plate bear gentle depressions or other types of muscle attachments (0); or they house deep pockets for such attachments (1). The derived state is present in Dissorophus and Broiliellus among the dissorophoids, but has also a wide distribution in stereospondyls (Watson, 1962; Yates and Warren, 2000).

66. Pointed snout. The tip of the snout is of various width but parabolic to squareshaped (0); or it is pointed (1). In dissorophoids, the pointed snout is shared by Dissorophus and Broiliellus brevis (Schoch, 2012).

67. Interclavicle. (0) Interclavicle rhomboidal, at least two times longer than wide. (1) Interclavicle with abbreviated anterior and posterior ends and as wide as long. (2) Interclavicle much shorter than wide, without anterior process. State 2 is probably derived from state 1, and present throughout branchiosaurids. Witzmann and Voigt (2015) have confirmed this coding in a recent review. 
68. Supinator process. Anterodistal region of humerus with supinator process (0); or lacking such process (1). As emphasized by Milner (1990), trematopids share a supinator with more primitive temnospondyls, whereas dissorophids, amphibamids, and branchiosaurids lack it. Adult specimens of Micromelerpeton also have a supinator (Boy, 1995).

69. Entepicondylar foramen. Distal end of humerus pierced by an entepicondylar foramen (0); or lacking such opening (1). This is a consistent post-Dendrysekos feature (Milner, 1990).

70. Dorsal eminences. Skull table without major raised areas other than ornamenting ridges (0); or with elevated eminences on the frontal, parietal, postfrontal, and postparietal (1). The apomorphic state is confined to the three species of Broiliellus (Schoch, 2012).

71. Anterior trunk ribs. The anterior trunk ribs have widened ends (0), or are simple rods without distal expansion (1). The apomorphic character-state is present in the amphibamids Platyrhinops, Amphibamus, and Eoscopus (Schoch and Milner, 2008).

72. Ventral bony scales. Spindle-shaped ventral scales fully ossified (0), or absent (1). This is only recognized in large specimens, where osteoderms are fully formed (Boy 1987; Witzmann 2007). The plesiomorphic character state is present only in Branchiosaurus, while all other branchiosaurids lack spindle-shaped ventral osteoderms in adult stages (Schoch and Milner, 2004, 2008).

73. Branchial dentition. Primitively, there are four rows of branchial denticles which are attached to rectangular or oval platelets bearing a total of 3-10 denticles that form a ratchet, as two of the outgroups indicate (0), or there are six rows of isolated denticles with a single point, or multi-ended (1) (Boy, 1972; Schoch and Milner, 2008). The branchial ossicles of amphibamids are still unknown, even in the larval specimens referred to Amphibamus grandiceps by Milner (1982).

74. Basipterygoid joint. Ossified (0), or unossified (1). The derived state is found in larval-neotenic micromelerpetids and branchiosaurids, but also occurs in adult stereospondylomorphs (Boy, 1988; Witzmann and Schoch, 2006).

75. Humerus: morphology. The humerus length:waist ratio is 3:4 in Dendrysekos, Balanerpeton, Sclerocephalus (0); 4:6 in Micromelerpeton and the branchiosaurid Schoenfelderpeton (1), and 6:10 in amphibamids and most branchiosaurids (2). We admit that this character may be problematic when the profound ontogenetic changes in large temnospondyls are considered (e.g., Pawley and Warren, 2006), but for comparisons among small dissorophoids and especially branchiosaurids it does appear to be informative (Schoch and Milner, 2008). 
76. Presacral count: reduction. Primitively a total of 24 presacral vertebrae are present (0), while fewer than 24 presacrals is clearly derived (1). All branchiosaurid species have fewer than 24 presacrals as an average, and most have only around 20 (Boy, 1972, 1978, 1987). The reduction of the presacral count to fewer than 24 is observed in the Amphibamidae as well, but there it is clearly a derived characterstate characterizing Amphibamus grandiceps (Milner, 1982) and Micropholis stowi (Schoch and Rubidge, 1995), while the primitive condition for the family is 24 presacrals (Daly, 1994; Clack and Milner, 2010). A further derived state is the reduction to 17 presacrals or fewer, which is shared by Gerobatrachus and lissamphibians (2).

77. Presacral count: elevation. Twenty-four presacral vertebrae (0), or clearly higher count of 26-29 (1). This condition is obviously a separate evolutionary pattern, confined to the Micromelerpetidae, which is why it is here defined as a separate character (Schoch and Rubidge, 2005).

78. Bicuspidity. The crowns of marginal and palatal teeth are primitively monocuspid (0), but are clearly bicuspid, with the cusps oriented lingually-labially (1), the derived state shared by some derived amphibamids and lissamphibians (Bolt, 1977).

79. Pedicely. The crowns of marginal and palatal teeth are primitively conical and continuous with the base (0), but are separated from the base by a zone in derived amphibamids, which is called pedicely (1) (Parsons and Williams, 1962; Bolt, 1969, 1977, 1979).

80. Width of palatine and ectopterygoid. These elements are either attached to the maxilla throughout their lateral margins (0), or the ectopterygoid and the posterior portion of the palatine are well separated from the maxilla, with the palatine attaining a Y-shaped outline (1). This character forms the second robust support for the monophyly of the Branchiosauridae. In addition to its uniqueness among temnospondyls, this state differs essentially from the derived condition in amphibamids where the palatine and ectopterygoid are extremely slender but attached to the maxilla, giving distinctly wider interpterygoid vacuities than in branchiosaurids or dissorophids, for instance. The peculiar branchiosaurid condition is acquired early in development (Schoch, 1992) and was maintained well into the largest growth stages (Boy, 1978; Werneburg, 1991).

81. Ectopterygoid. This forms a slender elongate element which bears teeth in the primitive condition, retained in branchiosaurids (0). In Schoenfelderpeton and Doleserpeton, it is reduced to a thin edentulous strut (1) (modified from Schoch and Rubidge, 2005).

82. Width of maxillary shelf. The main dentigerous body of the maxilla is slender in the primitive condition (0), while in Apateon it is markedly broadened, especially in adult and/or large specimens (Schoch and Milner, 2008). 
83. Postorbital separated from supratemporal. In the primitive condition, the supratemporal has a broad suture with the postorbital (0). In Leptorophus and Schoenfelderpeton, a posterolateral projection of the postfrontal keeps the tiny postorbital apart from the supratemporal (1), see Boy (1986) and Werneburg (2001).

84. Pubis. Ossified (0), or unossified (1) (Schoch, 2018).

85. Intercentra. In the primitive condition of temnospondyls, intercentra are wedgeshaped (0); in trematopids, they are dorsally enlarged (1) (Schoch, 2012).

86. Pleurocentra. Confined to the dorsal portion of the vertebral centrum (0), reaching ventrally down the flanks (1), forming short, closed rings (2), forming elongate cylinders (3) (modified from Schoch and Rubidge, 2005).

87. Otic notch. Present (0), absent (1). An enlarged semilunar squamosal embayment is present in all early and basal temnospondyls, as well as the vast majority of dissorophoids. The derived state is established in most dvinosaurians (incipiently present in Trimerorhachis) (Schoch and Milner, 2014).

88. Trunk ribs. Moderately long and curved (<length of three vertebrae) (0), or short and straight ( $<3$ vertebrae) (Schoch and Rubidge, 2005).

89. Choana. (0) Choana narrow, forming an elongated oval with parallel, parasagittal lateral and medial margins. (1) Choana expanded anteromedially (Schoch, 1998; Schoch and Rubidge, 2005). The derived state is not present in branchiosaurids and some amphibamids, but in Micropholis, Amphibamus, Doleserpeton, Gerobatrachus, and lissamphibians.

90. Cleithrum: head. (0) Head of the cleithrum aligned along the anterior rim of the scapula. (1) Cleithrum with posterodorsally enlarged head, wrapping around scapula dorsally (Schoch and Rubidge, 2005).

91. Cleithrum: size. (0) Cleithrum with large dorsal head much wider than shaft. (1) Cleithrum forming a simple rod, without any head (Schoch and Rubidge, 2005).

92. Scapula. (0) Scapula forming a low or moderately high element, depending on degree of ossification, about two times longer than wide. (1) Scapula dorsally much extended, being three to four times longer than wide (Schoch and Rubidge, 2005).

93. Basioccipital and supraoccipital. Present (0), or absent (1). The derived state characterizes all lissamphibians and adult branchiosaurids (Schoch and Milner, 2004). In the extant taxa, neither bone nor cartilage is formed in these regions, indicating that it is not simply the failure of a cartilage to ossify, but the complete 
loss of the element. Instead, the basisphenoid, which is also absent is extant amphibians, is retained in branchiosaurids albeit incompletely ossified (Boy, 1972).

94. Contact maxilla-quadratojugal. These are either sutured in early ontogenetic stages (0), or growth of the maxilla is slowed down with late suturing (1), or elements fail to meet, leaving a gap in the cheek (2). The three states are obviously correlated with ontogenetic character transformation $(2>1>0)$, and outgroup comparison indicates clearly that the adult state $(0)$ is the primitive condition (Boy, 1987).

95. Medial suture of supratemporal. The supratemporal is wide with a straight or convex medial suture (0), or much narrower and medially concave (1). The derived state is established in Leptorophus and Schoenfelderpeton (Schoch and Milner, 2008).

96. Shape of supratemporal. Primitively, the anterior portion of the supratemporal is blunt and not much narrower than the greatest width of the element (0). In most Melanerpeton, Leptorophus and Schoenfelderpeton, the anterior end is pointed to give the element a triangular outline; the squamosal compensates this space with a medial projection (1) (Schoch and Milner, 2008).

97. Jugal anteriorly abbreviated. Irrespective of its lost contact to the lacrimal, the jugal may be either anteriorly long (0), or it may lack the anterior process and end bluntly (1).

98. Intercentra. Present (0), or absent (1) (Anderson et al., 2008a).

99. Medial quadratojugal process. Absent (0), present (1) (Boy, 1972).

100. Pterygoid: slender. Primitively, the pterygoid has wide tooth-bearing palatine and basipterygoid rami (0), in the derived condition, the pterygoid has extremely thin, bar-like rami (1). The derived condition is established in branchiosaurids, Gerobatrachus, Triadobatrachus, and Karaurus (Schoch, 2018).

101. Circumorbital bones: shape. Plate-like (0), extremely thin, reduced to margin of orbit (1) (Modified from Anderson et al., 2008b).

102. Vomerine dentition. Single row of larger teeth including tusk pair (0), or patch of tiny denticles (1). The derived state is found in Doleserpeton, Amphibamus, and Gerobatrachus, whereas branchiosaurids have the plesiomorphic state. This character is confined to the region of the posterolateral branch of the vomer, whereas character (7) concerns tooth patches on the main portion of the element, the anteromedial plate (Bolt, 1979). 
103. Premaxilla: ascending process. Plate-like, aligned posterodorsally (0), or thin and rod-like, oriented posteromedially (1). The derived state is present only in Gerobatrachus and batrachians (Lebedkina, 1979).

104. Preorbital region: width. Of variable width, but with long axis of lacrimal aligned parasagittally (0), or much widened, and lacrimal aligned nearly transversely (1) (Schoch, 2018).

105. Ectopterygoid. Present (0), or absent (1). Doleserpeton retains an extremely thin, edentulous ectopterygoid (Sigurdsen and Bolt, 2010).

106. Postfrontal, postorbital, jugal, supratemporal, postparietal, tabular. Present (0), or absent (1) (Schoch, 2014d).

107. Cleithrum. Present (0), or absent (1). The derived state is confined to lissamphibians (batrachians in the present analysis). The presence of a cleithrum in anurans is highly controversial (Havelovká and Roček, 2006), and has therefore been coded as absent here.

108. Interclavicle. Present (0), or absent (1). The derived state is confined to lissamphibians (batrachians in the present analysis).

109. Basisphenoid. Present (0) or absent (1) (NEW).

110. Exoccipital foramen for cranial nerve XII. Present (0) or absent (1) (NEW).

111. Sphenethmoid. Sphenethmoid is either present as a single, medially positioned entity with the floor present (0), or the floor is absent and the sphenethmoid is present as two elements (1) (NEW). 


\title{
Appendix B: Matrix for Chapter 2
}

\author{
Acanthostomatops \\ 0010000000000001000000100000101001101101010000000000 \\ $00000001001 ? 0000101 ?$ ? 0 ? 00000000000000000000000000000000 \\ 0000000000000000 ?? ?
}

\section{Acheloma}

31100000000001101011110010011111111011000011111111100 $0000200100010000001010000 ? 0000000000000010001010100000$ 101000000000000 ? 0

\section{Amphibamus}

$1101110010111110110000010000011011000000 ? 00010000$ $001110010000000000111010 ? 02 ? 011010000020110101000$ 10101110000000 ?? ?

Anconastes

11 ? 000 ???? $001101011110010001101100 ? 0 ? 0101$ ? 010111 00111001 ? 00 ? 00000 ????0???0???000?00???0?1???000?0 ? 100 ? 00000 ?? 0 ??

\section{Apateon}

?? $01011010001100110010010 ? 00001000000000010000 ? 0000$ 00111001000000000021100111210 ?? 10101010201 ? 010101000 101111000000000 ?? ?

Ascaphus (Frogs)

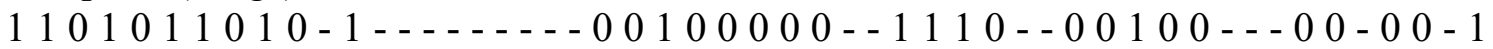

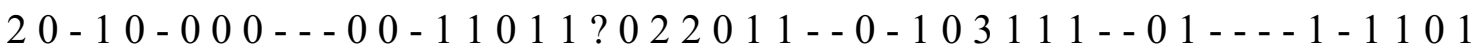

1111111111

\section{Aspidosaurus}

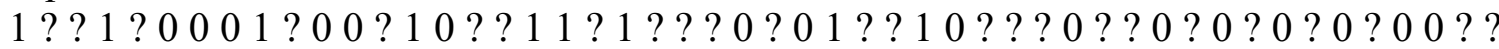

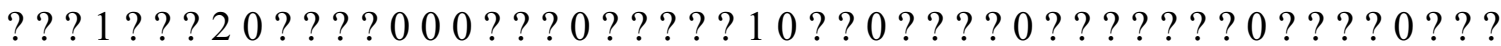
00 ? ? 0?? 0 ??

\section{Branchierpeton}

$1 ? 1010101000 ? 100100010110000011000000010 ? 000 ? 0000$ $002100010000000000111000001001000000010 ? 0000000 ? 000$ 101000000000 ??? 


\section{Branchiosaurus}

??01011010001100110000010?00001000000101000?0000 $00211001000000000011100011200 ? ? 10101 ? ? 010010 ? 100$ $1 ? 1110000000000$ ??

\section{Brevidorsum}

11 ???????????101111111?1101??100??????10???0?0?0?10

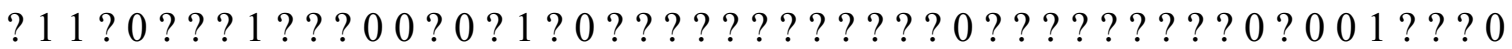
?? 0 ? 0? ? 0 ? ?

\section{Broiliellus}

11 ? 1?0??11011101011111?10?0?1010?010?100000000?000 $01111011111101011 ? 11100 ? 00 ? 01000000000001010100001$ $01000000000000 ? ?$ ?

\section{Cacops morrisi}

$1111 ? 0001100110111111011010111100001010001000002010$ $1101211112110100002110000 ? 0000000000000000010101000001$ 01000000000000 ?? ?

\section{Conjunctio}

$11 ? 1 ? 0 ? 01100 ? 101111110 ? 101011110 ? 01 ? ? 2010 ? 0 ? 0 ? 001$ $1111 ? 111 ? 1$ ? 0100000 ?? ? $0000 ? 00 ? 0000000000000101010100001$ $010000000000000 ?$ ?

\section{Dendrysekos}

0000000000000000000000100000000000000000010000000000000 $000000000000000000000000 ? 000000000000000000000000000$ $0000000000000000 ? 0$ ?

\section{Dissorophus}

$11 ? 1 ? 0 ? ? 11011101011111 ? 10 ? 011000 ? 010020000000000$ ? $01111101111110011111110000 ? 00 ? 00000000000000101010000$ 1010000000000000 ??

\section{Doleserpeton}

1110110010111110110010110000111011000000100010000 $001110010000000000111010 ? 02 ? 011000000020110101000$ 101011000000000

\section{Ecolsonia}

$211000 ? 00000110101111001000111111010 ? 11000002101$ $101110010001000000101000 ? 00 ? 00000000100 ? 01010000$ $1001000000000000 ? ?$ ? 


\section{Eimerisaurus}

$1 ? 10101010001100000010110000010000000201000 ? 0000$ $0021000100000000001110000100100000010 ? 000000 ? 000$ 1 ? 1000000000000 ???

\section{Eocaecilia}

? $00011101011---0$ ? - $0010 ? 000 ? 00-0110-0200000--00000$ $21-0-1000$ ? $000000-11010$ ? $0220110-0$ ? $103110--0110010$ ? 110101011111

\section{Eoscopus}

$1100000110111100010010110000000000000000000 ? 0000$ $001110010000000000111010 ? 02000000000010100001000$ $100101000000000 ? ?$ ?

\section{Eryopidae}

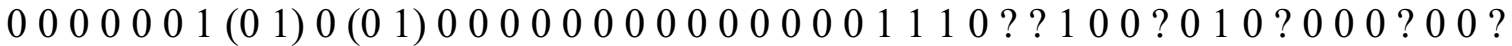

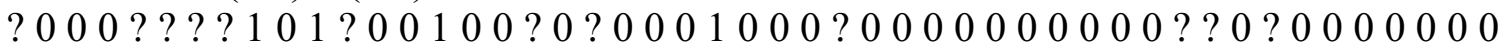
000 ? ? 001 ? ? 00 ? ? ? 000

\section{Fedexia}

11 ????????00?? 01011110010001111 ????0??1000000? 000 0111001000 ? 000000 ?? ? 000 ? 00 ? 00000000 ?? 0 ? 0??? ? 000010 $1000000000000 ? 0$

\section{Georgenthalia}

1 ? $00011010 ? 1 ? 10011001011000000000 ? 000101000 ? 0000$ 001120010000000000 ? 110 ?? ? 0??000???0???0???????000 $1001 ? 1 ? 000000$ ???

\section{Gerobatrachus}

$11 ? ? ? ? 10100111000100100 ? 0000001001000101000 ? 0000$ $001120010000000000 ? 1100 ? ? ? ? 20000010103010010 ? 000$ $10 ? 1111100000 ? ?$ ?

\section{Hynobius (Salamanders)}

? $011011010-1-\ldots-1--10101001-001-0--20100--$ - ? $0100-1$ $20-10-000--00-11011 ? 022011--0-103111--01---1-1101$ 111111111

\section{Kamacops}

$1111000011001101 ? 11110 ? 10 ? 011 ? ? 0001010000 ? 0 ? 2000$

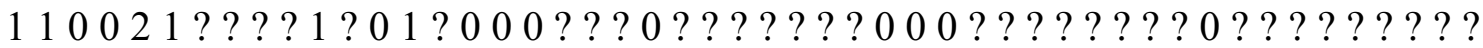
? ? ? 0 ? ? 00 ? ? ? ? ? 


\section{Karaurus}

? $011011010-1---$ - ? - - 110 ? $01001--0110--201000--100000$ $120-10000000000-1101110220110-001031110-01100-111$ 10111111 ??

\section{Leptorophus}

? ? $00011010001100010010010000001000000201000 ? 0000$ $00111001000000000021100111210 ?$ ? 10111 ?? 010010 ? 011 1 ? 1111000000000 ?? ?

\section{Limnogyrinus}

?? $1010101000 ? ? 00000000110000000000000010 ? 000 ? 0000$ $0021000100000000001110000100100000010 ? 000000 ? 000$ 10100000000000 ?? ?

\section{Micromelerpeton}

1 ? $1000011000 ? 1000000101100000100000 ? ? 00100000000$ $00110001000000000000100001101000000101000000 ? 000$ 10100000000000 ???

\section{Micropholis}

$1110100110111101010010110000000011000000000 ? 0000$ $002110010000000000111000 ? 021000000000101 ? 0101000$ $101000000000000 ? ? 0$

\section{Pasawioops}

$111000011011110001001001000000001 ? 00000 ? 000 ? 0000$ 002110010000000000 ?? 10 ? ? ? 02 ? 00000000 ? ? 0? 0?? ? ? 0001 0100000000000000 ?

\section{Perryella}

$111010001000 ? ? 0000001000000000010 ? 00000 ? 000 ? 0000$ $001000010000000000101000 ? 00000000000000 ? 00000000$ $10010000000000000 ?$ ?

\section{Phonerpeton}

$1111 ? 0000000111101111001001111111010001111010100$ $100110110001000000 ? 01000 ? 0000000000010010 ? ? ? 0000$ $1010000000000000 ? 0$

\section{Platyhystrix}

???1?0??10?0?101011111?0?10?10000??0?0???0?0?0?00?

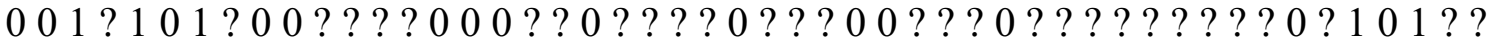
? 0 ? ? ? 0 ? ? ? 000 


\section{Platyrhinops}

$1100100010111110110001 ? 1000011101000 ? 00000000000$ $000110010000000000111000 ? 0200100000001010000 ? 000$ $1001000000000000 ? 0$

\section{Plemmyradytes}

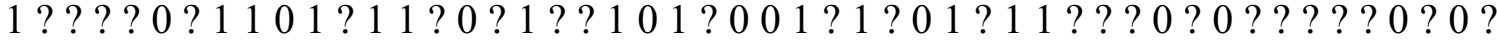

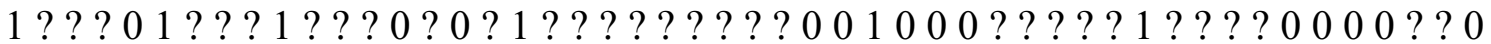
00000 ? ?????

\section{Reiszerpeton}

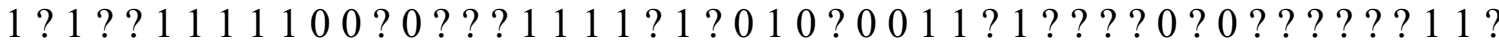

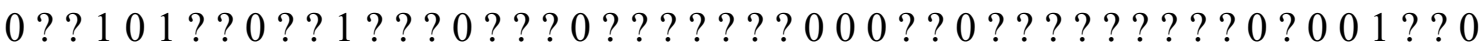
$0100 ? 0$ ? ? 0 ??

Rhinatrema (Caecilians)

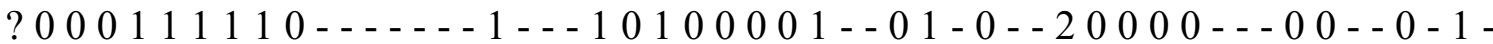

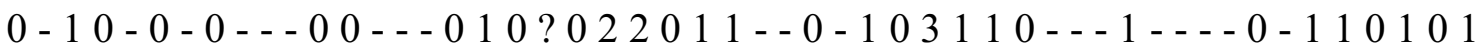
01111111

Rio Arriba taxon

$11 ? 10 ? 001100 ? 10111111 ?$ ? $101011010 ? 01 ? ? 2010 ? 0 ? 0 ? 001$ 1111 ? 0 ??? 1???0000???0?????10??0??0???0???????000?? ? 000 ? ? 0?????

\section{Rubeostratilia}

$1 ? 1010011011$ ? 0000011101 ? 0?0?0010?11???0????10?0?? ?1??001????0??0?0???????0???001000?????1????0000?? $000000000 ? ? ? ? ? 0$

\section{Scapanops}

?1???????????11111111111?10101101??????2010 ? 0? 0?00 ? 0

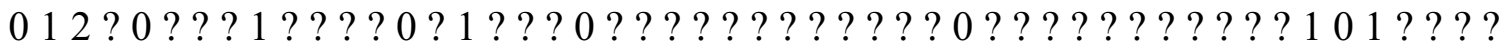
? 0 ?? 0 ?????

\section{Schoenfelderpeton}

?? ?00011010001100010010010100001000000201000?0000 $00211001000000000021100111110 ? ? 11111$ ??010010?111 $1 ? 1111000000000 ? ? ?$

\section{Sclerocephalus}

0000000000000000000000001010000000010000000000000 000000010000000000001000010000000000000000000000 00000000000000 ? ? 0 


\section{Tambachia}

$11 ? 000 ? 00000110100111001000111101010 ? 01011010101$

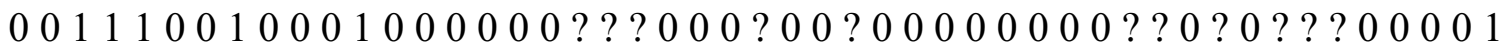
0100000000000 ?? ?

\section{Tersomius}

111000011011110001001011000000001000000000010000 0021100100000000001010 ??? 02 ? 010000000101 ??? 01000 10100000000000000

\section{Triadobatrachus}

110101 ? 010 - 1 - - - - - - 100 ? 000000 - - ? ? 10 - - 00 ? 000 - - ? 000011 $20-10000000000-11011 ? 0220110-001030110-01100-1111$ 0111111 ? ? 1

\section{Trimerorhachis}

000100100000000100000000000000000000010000000000 002000000000000000001000000010000001001000000000 000000000000000000 


\section{Appendix C: Taxa list for Chapter 2}

Table C1. A list of taxa coded for the phylogenetic analyses in Chapter 2. Citations for taxa coded from the literature are below.

\begin{tabular}{|c|c|c|c|}
\hline $\begin{array}{l}\text { Acanthostomatops } \\
\text { vorax }[1]\end{array}$ & $\begin{array}{l}\text { Acheloma } \\
\text { cummiunsi [2] }\end{array}$ & $\begin{array}{l}\text { Admiral taxon } \\
\text { (Scapanops) [3] }\end{array}$ & $\begin{array}{l}\text { Amphibamus } \\
\text { (YPM 794) }\end{array}$ \\
\hline Anconastes [4] & $\begin{array}{l}\text { Apateon gacilis } \\
\text { [5] }\end{array}$ & $\begin{array}{l}\text { Ascaphus [6] } \\
\text { (representative } \\
\text { frog species) }\end{array}$ & Aspidosaurus [7] \\
\hline $\begin{array}{l}\text { Branchierpeton } \\
\text { amblystomum [8] }\end{array}$ & $\begin{array}{l}\text { Branchiosaurus } \\
\text { [9] }\end{array}$ & Brevidorsum [3] & Broiliellus [3] \\
\hline Cacops [10] & Conjunctio [3] & $\begin{array}{l}\text { Dendrysekos } \\
{[11,12]}\end{array}$ & Dissorophus [13] \\
\hline Doleserpeton [14] & Ecolsonia $[15]$ & $\begin{array}{l}\text { Eimerisaurus } \\
\text { graumanni [16] }\end{array}$ & Eocaecilia $[6,17]$ \\
\hline Eoscopus [18] & Eryopidae $[19,20]$ & Fedexia $[21]$ & $\begin{array}{l}\text { Georgenthalia } \\
{[22]}\end{array}$ \\
\hline Gerobatrachus [23] & $\begin{array}{l}\text { Hynobius [6] } \\
\text { (representative } \\
\text { salamander } \\
\text { species) }\end{array}$ & Kamacops [24] & Karaurus $[6,25]$ \\
\hline Leptorophus [26,27] & $\begin{array}{l}\text { Limnogyrinus } \\
\text { elegans }[28]\end{array}$ & $\begin{array}{l}\text { Micromelerpeton } \\
\text { [29] }\end{array}$ & Micropholis [30] \\
\hline $\begin{array}{l}\text { Pasawioops } M C Z \\
\text { (MCZ 1415, OMNH } \\
73019, \text { CT data) }\end{array}$ & $\begin{array}{l}\text { Perryella olsoni } \\
\text { [31] }\end{array}$ & Phonerpeton [32] & Platyhystrix [33] \\
\hline Platyrhinops [34] & $\begin{array}{l}\text { Plemmyradytes } \\
{[35]}\end{array}$ & $\begin{array}{l}\text { Reiszerpeton } \\
(\mathrm{MCZ} 1911)[36]\end{array}$ & $\begin{array}{l}\text { Rhinatrema } \\
\text { (representative } \\
\text { caecilian species) } \\
{[6]}\end{array}$ \\
\hline Rio Arriba taxon [3] & $\begin{array}{l}\text { Rubeostratilia } \\
{[37]}\end{array}$ & $\begin{array}{l}\text { Schoenfelderpeton } \\
{[26]}\end{array}$ & $\begin{array}{l}\text { Sclerocephalus } \\
\text { [38] }\end{array}$ \\
\hline Tambachia [39] & Tersomius [40] & $\begin{array}{l}\text { Triadobatrachus } \\
{[6,41,42]}\end{array}$ & $\begin{array}{l}\text { Trimerorhachis } \\
\text { insignis [43] }\end{array}$ \\
\hline
\end{tabular}


1. Witzmann F, Schoch RR. Skeletal development of the temnospondyl Acanthostomatops vorax from the Lower Permian Döhlen Basin of Saxony. Earth Environ Sci Trans R Soc Edinb. 2006;96: 365-185.

2. Polley BP, Reisz RR. A new Lower Permian trematopid (Temnospondyli: Dissorophoidea) from Richards Spur, Oklahoma. Zool J Linn Soc. 2011;161: 789815.

3. Carroll RL. Early evolution of the dissorophid amphibians. Bull Mus Comp Zool. 1964;131: 161-250.

4. Berman DS, Reisz RR, Eberth DA. A new genus and species of trematopid amphibian from the Late Pennsylvanian of north-central New Mexico. J Vert Paleontol. 1987;7: 252-269.

5. Fröbisch NB, Schoch RR. The largest specimen of Apateon and the life history pathway of neoteny in the Paleozoic temnospondyl family Branchiosauridae. Foss Rec. 2009;12: 83-90.

6. Maddin HC, Jenkins FA Jr, Anderson JS. The braincase of Eocaecilia micropodia (Lissamphibia, Gymnophiona) and the origin of caecilians. PLOS One. 2012;7: e50743.

7. Berman DS, Lucas SG. Aspidosaurus binasser (Amphibia, Temnospondyli), a new species of Dissorophidae from the Lower Permian of Texas. Ann Carnegie. 2003;72: 241-262.

8. Schoch RR, Milner AR. Handbuch Der Paläoherpetologie: Encyclopedia of Paleoherpetology. Temnospondyli I/by Rainer R. Schoch and Andrew R. Milner. Pfeil; 2014.

9. Schoch RR, Milner AR. The intrarelationships and evolutionary history of the temnospondyl family Branchiosauridae. J Syst Palaeontol. 2008;6: 409-431.

10. Williston SW. Cacops, Desmospondylus; new genera of Permian vertebrates. Geol Soc Am Bull. 1910;21: 249-284.

11. Robinson J, Ahlberg PE, Koentges G. The braincase and middle ear region of Dendrerpeton acadianum (Tetrapoda: Temnospondyli). Zool J Linnean Soc. 2005;143: 577-597.

12. Holmes RB, Carroll RL, Reisz RR. The first articulated skeleton of Dendrerpeton acadianum (Temnospondyli, Dendrerpetontidae) from the Lower Pennsylvanian locality of Joggins, Nova Scotia, and a review of its relationships. J Vert Paleontol. 1998;18: 64-79. 
13. De Mar RE. The Permian labyrinthodont amphibian Dissorophus multicinctus, and adaptations and phylogeny of the family Dissorophidae. J Paleontol 1968;42: $1210-1242$.

14. Sigurdsen T, Bolt JR. The Lower Permian amphibamid Doleserpeton (Temnospondyli: Dissorophoidea), the interrelationships of amphibamids, and the origin of modern amphibians. J Vert Paleol. 2010;30: 1360-1377.

15. Berman DS, Reisz RR, Eberth DA. Ecolsonia cutlerensis, an Early Permian dissorophid amphibian from the Cutler Formation of north-central New Mexico. New Mexico Bureau of Mines and Mineral Resources 1985;191: 1-31.

16. Boy JA. Über die Micromelerpetontidae (Amphibia: Temnospondyli). 3. Eimerisaurus n. g.: Neues Jahrbuch für Geologie und Paläontologie, Abhandlungen. 2002;225: 425-452.

17. Jenkins FA Jr, Walsh DM, Carroll RL. Anatomy of Eocaecilia micropodia, a limbed caecilian of the Early Jurassic. Bull Mus Comp Zool. 2007;158: 285-365.

18. Daly E. Amphibamidae (Amphibia: Temnospondyli), with a description of a new genus from the upper Pennsylvanian of Kansas. Monogr Mus Nat Hist Univ Kans. 1994;85: 1-59.

19. Werneburg R, Lucas SG, Schneider JW, Rinehart LF. First Pennsylvanian Eryops (Temnospondyli) and its Permian record from New Mexico. Bull N M Mus Nat Hist Sci. 2010;49: 129-135.

20. Dempster WT. The braincase and endocranial cast of Eryops megacephalus (Cope). J Comp Neurol. 1935;62: 171-196.

21. Berman DS, Henrici AC, Brezinski DK, Kollar AD. A new trematopid amphibian (Temnospondyli: Dissorophoidea) from the Upper Pennsylvanian of western Pennsylvania: earliest record of terrestrial vertebrates responding to a warmer, drier climate. Ann Carnegie. 2009;78: 289-318.

22. Anderson JS, Henrici AC, Sumida SS, Martens T, Berman DS. Georgenthalia clavinasica, a new genus and species of dissorophoid temnospondyl from the Early Permian of Germany, and the relationships of the family Amphibamidae. J Vert Paleontol. 2008;28: 61-75.

23. Anderson JS, Reisz RR, Scott D, Fröbisch NB, Sumida SS. A stem batrachian from the Early Permian of Texas and the origin of frogs and salamanders. Nature. 2008;453: 515-518. 
24. Schoch RR. Studies on braincases of early tetrapods: Structure, morphological diversity, and phylogeny-2. Dissorophoids, eryopids, and stereospondyls. Neues Jahrbuch für Geologie und Paläontologie - Abhandlungen 1999;213: 289- 312.

25. Ivachnenko MF. Urodelans from the Triassic and Jurassic of Soviet central Asia. Paleontol J. 1978;12: 362-368.

26. Boy JA. Studien über die Branchiosauridae (Amphibia: Temnospondyli) 1. Neue und wenig bekannte Arten aus dem mitteleuropäischen Rotliegenden (? oberstes Karbon bis unteres Perm). Paläontologische Zeitschrift 1986;60: 131-166.

27. Schoch RR. First evidence of the branchiosaurid temnospondyl Leptorophus in the Early Permian of the Saar-Nahe Basin (SW Germany). Neues Jahrbuch für Geologie und Paläontologie - Abhandlungen 2014;272: 225-236.

28. Werneburg, R. Dissorophoiden (Amphibia, Rhachitomi) aus dem Westfal D (Oberkarbon) von Böhmen. Limnogyrinus elegans (Fritsch 1881): Zeitschrift für geologische Wissenschaften. 1994;22: 457-467.

29. Boy JA. Über die Micromelerpetontidae (Amphibia: Temnospondyli). 1. Morphologie und Paläoökologie des Micromelerpeton credneri (Unter-Perm; SWDeutschland). Paläontologische Zeitschrift. 1995;69: 429-457.

30. Schoch RR, Rubidge BS. The amphibamid Micropholis from the Lystrosaurus assemblage zone of South Africa. J Vert Paleontol. 2005;25: 502-522.

31. Ruta M, Bolt JR. A reassessment of the temnospondyl amphibian Perryella olsoni from the Lower Permian of Oklahoma. Earth Environ Sci Trans R Soc Edinb. 2006;97: 113-65.

32. Dilkes DW. A new trematopsid amphibian (Temnospondyli: Dissorophoidea) from the Lower Permian of Texas. J Vert Paleontol. 1990;10: 222-243.

33. Berman DS, Reisz RR, Fracasso MA. Skull of the Lower Permian dissorophid amphibian Platyhystrix rugosus. Ann Carnegie 1981;50: 391-416.

34. Clack JA, Milner AR. Morphology and systematics of the Pennsylvanian amphibian Platyrhinops lyelli (Amphibia: Temnospondyli). Earth Env Sci Trans R Soc. 2009;100: 275-295.

35. Huttenlocker AK, Pardo JD, Small BJ. Plemmyradytes shintoni, gen. et sp. nov., an Early Permian amphibamid (Temnospondyli: Dissorophoidea) from the Eskridge Formation, Nebraska. J Vert Paleontol. 2007;27: 316-328. 
36. Maddin HC, Fröbisch NB, Evans DC, Milner AR. Reappraisal of the Early Permian amphibamid Tersomius texensis and some referred material. CR Palevol. 2013;12: 447-461.

37. Bourget H, Anderson JS. A new amphibamid (Temnospondyli: Dissorophoidea) from the Early Permian of Texas. J Vert Paleontol. 2011;31: 32-49.

38. Schoch RR, Witzmann F. Osteology and relationships of the temnospondyl genus Sclerocephalus. Zool J Linnean Soc. 2009;157: 135-168.

39. Sumida SS, Berman DS, and Martens T. A new trematopid amphibian from the Lower Permian of central Germany. Palaeontol. 1998;41: 605-630.

40. Anderson JS, Bolt JR. New information on amphibamids (Tetrapoda, Temnospondyli) from Richards Spur (Fort Sill), Oklahoma. J Vert Paleontol. 2013;33: 553-567.

41. Ascarrunz E, Rage J-C, Legreneur P, Laurin M. Triadobatrachus massinoti, the earliest known lissamphibian (Vertebrata: Tetrapoda) re-examined by $\mu \mathrm{CT}$ scan, and the evolution of trunk length in batrachians. Contrib Zool. 2016;85: 201-234.

42. Rage JC, and Rocek Z. Redescription of Triadobatrachus massinoti (Piveteau, 1936) an anuran amphibian from the early Triassic. Palaeontographica 1989;206: $1-16$.

43. Milner AR, Schoch RR. Trimerorhachis (Amphibia: Temnospondyli) from the Lower Permian of Texas and New Mexico: Cranial osteology, taxonomy, and biostratigraphy: Neues Jahrbuch für Geologie und Paläontologie Abhandlungen. 2013;270: 91-128. 


\section{Appendix D: Somite injection specimen numbers for Chapter 3}

Table D1. Ambystoma mexicanum specimens injected with dextran-fluorescein to track somite development.

\begin{tabular}{|l|l|l|}
\hline & Number injected & Number that survived \\
\hline Somite 2 & 32 & 1 \\
\hline Somite 3 & 39 & 1 \\
\hline Somite 4 & 26 & 2 \\
\hline
\end{tabular}

Table D2. Ambystoma mexicanum specimens that underwent GFP-positive somite transplant surgery. Data set gifted by Hillary Maddin.

\begin{tabular}{|l|l|}
\hline & Specimens in data set \\
\hline Somite 2 & 1 \\
\hline Somite 3 & 2 \\
\hline Somite 4 & 1 \\
\hline
\end{tabular}

Table D3. Xenopus laevis specimens injected with dextran-fluorescein to track somite development.

\begin{tabular}{|l|l|l|}
\hline & Number injected & Number that survived \\
\hline Somite 2 & 27 & 4 \\
\hline Somite 3 & 24 & 0 \\
\hline Somite $2+3$ & 2 & 1 \\
\hline
\end{tabular}

****Survival here only counts specimens that continued to visibly fluoresce for weeks after injection and survived long enough to be fixed and sectioned. 


\section{Appendix E: Ambystoma mexicanum staging table specimen numbers}

Table E1. Ambystoma mexicanum specimens whole-mount cartilage and bone stained for the staging series.

\begin{tabular}{|l|l|l|l|}
\hline Stage & Total number of specimens & Stage & Total number of specimens \\
\hline $44 / 45$ & 7 & 52 & 10 \\
\hline 46 & 14 & 53 & 12 \\
\hline 47 & 12 & 54 & 10 \\
\hline 48 & 3 & 55 & 6 \\
\hline 49 & 12 & 56 & 19 \\
\hline 50 & 12 & 57 & 5 \\
\hline 51 & 6 & & \\
\hline
\end{tabular}

Table E2. Ambystoma mexicanum specimens whole-mount nerve stained.

\begin{tabular}{|l|l|}
\hline Stage & Total number of specimens \\
\hline 46 & 4 \\
\hline 47 & 3 \\
\hline 52 & 3 \\
\hline 53 & 4 \\
\hline 54 & 4 \\
\hline 57 & 3 \\
\hline
\end{tabular}




\section{Appendix F: Xenopus laevis staging table specimen numbers}

Table F1. Xenopus laevis specimens whole-mount cartilage and bone stained for the staging series.

\begin{tabular}{|l|l|l|l|}
\hline Stage & Total number of specimens & Stage & Total number of specimens \\
\hline 41 & 2 & 54 & 8 \\
\hline 42 & 2 & 55 & 11 \\
\hline 43 & 3 & 56 & 9 \\
\hline 44 & 4 & 57 & 8 \\
\hline 45 & 6 & 58 & 9 \\
\hline 46 & 4 & 59 & 3 \\
\hline 47 & 7 & 60 & 2 \\
\hline 48 & 3 & 61 & 2 \\
\hline 49 & 8 & 62 & 4 \\
\hline 50 & 2 & 63 & 3 \\
\hline 51 & 5 & 64 & 3 \\
\hline 52 & 2 & 65 & 4 \\
\hline 53 & 7 & 66 & 4 \\
\hline
\end{tabular}

Table F2. Xenopus laevis specimens whole-mount nerve stained.

\begin{tabular}{|l|l|}
\hline Stage & Total number of specimens \\
\hline 51 & 1 \\
\hline 55 & 6 \\
\hline 57 & 3 \\
\hline
\end{tabular}




\section{Appendix G: Ambystoma mexicanum exogenous and inhibited retinoic acid treatment experiment specimen numbers}

Table G1. Total number of Ambystoma mexicanum experimental animals exposed to exogenous retinoic acid. Treatments started between stages 28 to 35 .

\begin{tabular}{|l|l|l|l|l|}
\hline Treatment & $\begin{array}{l}\text { Total number of } \\
\text { specimens } \\
\text { treated that } \\
\text { survived to } \\
\text { hatching }\end{array}$ & $\begin{array}{l}\text { Total bone and } \\
\text { cartilage stained } \\
\text { specimens } \\
\text { (displayed } \\
\text { modified } \\
\text { phenotype) }\end{array}$ & $\begin{array}{l}\text { Total number } \\
\text { stained of nerve- } \\
\text { stained } \\
\text { specimens from } \\
\text { each treatment }\end{array}$ & $\begin{array}{l}\text { Total number of } \\
\text { control } \\
\text { specimens } \\
\text { (DMSO without } \\
\text { RA was added } \\
\text { to their water) }\end{array}$ \\
\hline $\begin{array}{l}0.01 \mu \mathrm{M} \\
\text { RA, } 4 \text { days }\end{array}$ & 22 & $20(13)$ & 2 & 30 \\
\hline $\begin{array}{l}0.01 \mu \mathrm{M} \\
\text { RA, 7 days }\end{array}$ & 44 & $41(17)$ & 3 & 40 \\
\hline
\end{tabular}

Table G2. Specimen numbers for Ambystoma mexicanum experimental animals where retinoic acid was inhibited via citral. Treatments started between stages 25 to 30 .

\begin{tabular}{|l|l|l|l|l|}
\hline Treatment & $\begin{array}{l}\text { Total number of } \\
\text { specimens } \\
\text { treated that } \\
\text { survived to } \\
\text { hatching }\end{array}$ & $\begin{array}{l}\text { Total bone and } \\
\text { cartilage stained } \\
\text { specimens } \\
\text { (displayed } \\
\text { modified } \\
\text { phenotype) }\end{array}$ & $\begin{array}{l}\text { Total number } \\
\text { stained of nerve- } \\
\text { stained } \\
\text { specimens from } \\
\text { each treatment }\end{array}$ & $\begin{array}{l}\text { Total number of } \\
\text { control } \\
\text { specimens } \\
\text { (DMSO without } \\
\text { citral was added } \\
\text { to their water) }\end{array}$ \\
\hline $\begin{array}{l}5 \mu \mathrm{M} \text { citral, } \\
2 \text { weeks }\end{array}$ & 5 & $3(2)$ & 0 & 10 \\
\hline $\begin{array}{l}10 \mu \mathrm{M} \\
\text { citral, } \\
\text { weeks }\end{array}$ & 5 & $3(2)$ & 0 & 4 \\
\hline $\begin{array}{l}15 \mu \mathrm{M} \\
\text { citral, } 2 \\
\text { weeks }\end{array}$ & 1 & 1 & 0 & 7 \\
\hline $\begin{array}{l}20 \mu \mathrm{M} \\
\text { citral, } 2 \\
\text { weeks }\end{array}$ & 9 & $7(7)$ & 2 & 2 \\
\hline
\end{tabular}


Table G3. Total number of Ambystoma mexicanum with GFP-positive somite transplants experimental animals exposed to exogenous retinoic acid or citral. Treatments started between stages 28 to 35 (exogenous retinoic acid) or between stages 25 and 30 (citral). Dataset gifted by Hillary Maddin.

\begin{tabular}{|l|l|l|}
\hline Somite & Treatment & Number of specimens \\
\hline 2 & $0.05 \mu \mathrm{M}$ RA, 14 days & 1 \\
\hline 3 & $0.01 \mu \mathrm{M}$ RA, 4 days & 1 \\
\hline 3 & $0.05 \mu \mathrm{M}$ RA, 7 days & 1 \\
\hline 3 & $0.05 \mu \mathrm{M}$ RA, 4 days & 1 \\
\hline 3 & $20 \mu \mathrm{M}$ citral, 14 days & 1 \\
\hline
\end{tabular}




\section{Appendix H: Xenopus laevis exogenous and inhibited retinoic acid treatment experiment specimen numbers}

Table H1. Total number of Xenopus laevis experimental animals exposed to exogenous retinoic acid. Treatments started between stages ten and eleven.

\begin{tabular}{|l|l|l|l|l|}
\hline Treatment & $\begin{array}{l}\text { Total number } \\
\text { of specimens } \\
\text { treated that } \\
\text { survived to } \\
\text { hatching }\end{array}$ & $\begin{array}{l}\text { Total bone } \\
\text { and cartilage } \\
\text { stained } \\
\text { specimens } \\
\text { (displayed } \\
\text { modified } \\
\text { phenotype) }\end{array}$ & $\begin{array}{l}\text { Total number } \\
\text { stained of } \\
\text { nerve-stained } \\
\text { specimens } \\
\text { from each } \\
\text { treatment }\end{array}$ & $\begin{array}{l}\text { Total number } \\
\text { of control } \\
\text { specimens } \\
\text { (DMSO } \\
\text { without RA } \\
\text { was added to } \\
\text { their water) }\end{array}$ \\
\hline $\begin{array}{l}0.01 \mu \mathrm{M} \text { RA, } 30 \\
\text { minutes }\end{array}$ & 1 & 0 & 0 & 10 \\
\hline $\begin{array}{l}0.01 \mu \mathrm{M} \text { RA, } 60 \\
\text { minutes }\end{array}$ & 30 & $6(6)$ & 0 & 7 \\
\hline $\begin{array}{l}0.01 \mu \mathrm{M} \mathrm{RA}, \\
\text { overnight }\end{array}$ & 8 & $5(5)$ & 3 & 15 \\
\hline $\begin{array}{l}0.001 \mu \mathrm{M} \mathrm{RA}, 60 \\
\text { minutes }\end{array}$ & 7 & $6(6)$ & 0 & 10 \\
\hline $\begin{array}{l}0.001 \mu \mathrm{M} \mathrm{RA}, \\
\text { overnight }\end{array}$ & 6 & 4 & 3 & \\
\hline
\end{tabular}


Table H2. Total number of Xenopus laevis experimental animals where retinoic acid was inhibited via citral. Treatments started between stages ten and eleven.

\begin{tabular}{|l|l|l|l|l|}
\hline Treatment & $\begin{array}{l}\text { Total number } \\
\text { of specimens } \\
\text { treated that } \\
\text { survived to } \\
\text { hatching } \\
\text { and cartilage } \\
\text { stained } \\
\text { specimens } \\
\text { displayed } \\
\text { modified } \\
\text { phenotype) }\end{array}$ & $\begin{array}{l}\text { Total bone } \\
\text { stained of } \\
\text { nerve-stained } \\
\text { specimens } \\
\text { from each } \\
\text { treatment }\end{array}$ & $\begin{array}{l}\text { Total number } \\
\text { of control } \\
\text { specimens } \\
\text { (DMSO } \\
\text { without citral } \\
\text { was added to } \\
\text { their water) }\end{array}$ \\
\hline $\begin{array}{l}1 \mu \mathrm{M} \text { citral, 24 } \\
\text { hours }\end{array}$ & 5 & 2 & 0 & 10 \\
\hline $\begin{array}{l}2.5 \mu \mathrm{M} \text { citral, 24 } \\
\text { hours }\end{array}$ & 23 & $8(4)$ & 5 & 20 \\
\hline $\begin{array}{l}5 \mu \mathrm{M} \text { citral, 24 } \\
\text { hours }\end{array}$ & 14 & $7(5)$ & 0 & 20 \\
\hline $\begin{array}{l}10 \mu \mathrm{M} \text { citral, } 24 \\
\text { hours }\end{array}$ & 22 & $4(4)$ & 0 & 5 \\
\hline $\begin{array}{l}15 \mu \mathrm{M} \text { citral, } 24 \\
\text { hours }\end{array}$ & 8 & $1(1)$ & 0 & 5 \\
\hline $\begin{array}{l}20 \mu \mathrm{M} \text { citral, 24 } \\
\text { hours }\end{array}$ & 3 & $1(1)$ & 1 & 5 \\
\hline
\end{tabular}




\section{References}

Alastair, M., Linda, A.-M., Alex, G., Mark, F. and Robb, K. (1997). HOXD4 and regulation of the group 4 paralog genes. Development, 124(16), 3135.

Alberch, P. (1989). Development and the evolution of amphibian metamorphosis. Fortschritte der Zoologie, 35, 163-173.

Altig, R. (1969). Notes on the ontogeny of the osseous cranium of Ascaphus truei. Herpetologica, 25(1), 59-62.

Anderson, J. S., Reisz, R. R., Scott, D., Fröbisch, N. B., and Sumida, S. S. (2008a). A stem batrachian from the Early Permian of Texas and the origin of frogs and salamanders. Nature, 453(7194), 515-518.

Anderson, J. S., Henrici, A. C., Sumida, S. S., Martens, T., and Berman, D. S. (2008b). Georgenthalia clavinasica, a new genus and species of dissorophoid temnospondyl from the Early Permian of Germany, and the relationships of the family Amphibamidae. Journal of Vertebrate Paleontology, 28(1), 61-75.

Anderson, J. S., and Bolt, J. R. (2013). New information on amphibamids (Tetrapoda, Temnospondyli) from Richards Spur (Fort Sill), Oklahoma, 33(3), 553-567.

Aoyama, H., and Asamoto, K. (1988). Determination of somite cells: independence of cell differentiation and morphogenesis. Development, 104(1), 15-28.

Aoyama, H., and Asamoto, K. (2000). The developmental fate of the rostral/caudal half of a somite for vertebra and rib formation: experimental confirmation of the resegmentation theory using chick-quail chimeras. Mechanisms of Development, 99(12), 71-82.

Armstrong, J. B., and Malacinski, G. M. (1989). Developmental biology of the axolotl. Oxford: Oxford University Press.

Ascarrunz, E., Rage, J.-C., Legreneur, P., and Laurin, M. (2016). Triadobatrachus massinoti, the earliest known lissamphibian (Vertebrata: Tetrapoda) re-examined by $\mu \mathrm{CT}$ scan, and the evolution of trunk length in batrachians. Contributions to Zoology, $85(2), 201-234$.

Atkins, J. B., and Franz-Odendaal, T. A. (2016). The evolutionary and morphological history of the parasphenoid bone in vertebrates. Acta Zoologica, 97(2), 255-263.

Augier, M. (1931). Squelette cephalique. In: Poirier P, Charpy A, editors. Traité d'anatomie humaine, vol. 1. Paris: Masson et Cie; pp. 19. 
Bagnall, K. M., Higgins, S. J., and Sanders, E. J. (1988). The contribution made by a single somite to the vertebral column: experimental evidence in support of resegmentation using the chick-quail chimaera model. Development, 103(1), 69-85.

Barnard, J. W. (1940). The hypoglossal complex of vertebrates. Journal of Comparative Neurology, 72(3), 489-524.

Begemann, G., and Meyer, A. (2001). Hindbrain patterning revisited: Timing and effects of retinoic acid signalling. Bioessays, 23(11), 981-986.

Bel-Vialar, S., Itasaki, N. and Krumlauf, R. (2002). Initiating Hox gene expression: in the early chick neural tube differential sensitivity to FGF and RA signaling subdivides the HoxB genes in two distinct groups. Development, 129(22), 5103-5115.

Berman, D. S. (2000). Origin and early evolution of the amniote occiput. Journal of Paleontology, 74(05), 938-956.

Berman, D. S., Reisz, R. R., and Fracasso, M. A. (1981). Skull of the Lower Permian dissorophid amphibian Platyhystrix rugosus. Carnegie Museum of Natural History, 50, 391-416.

Berman, D. S., Reisz, R. R., and Eberth, D. A. (1985). Ecolsonia cutlerensis, an Early Permian dissorophid amphibian from the Cutler Formation of north-central New Mexico. New Mexico Bureau of Mines and Mineral Resources, 191, 1-31.

Berman, D. S., Reisz, R. R., and Eberth, D. A. (1987). A new genus and species of trematopid amphibian from the Late Pennsylvanian of north-central New Mexico. Journal of Vertebrate Paleontology, 7(3), 252-269.

Berman, D. S., and Lucas, S. G. (2003). Aspidosaurus binasser (Amphibia, Temnospondyli), a new species of Dissorophidae from the Lower Permian of Texas. Annals-Carnegie Museum Pittsburgh, 72(4), 241-262.

Berman, D. S., Henrici, A. C., Brezinski, D. K., and Kollar, A. D. (2009). A new trematopid amphibian (Temnospondyli: Dissorophoidea) from the Upper Pennsylvanian of western Pennsylvania: earliest record of terrestrial vertebrates responding to a warmer, drier climate. Annals of Carnegie Museum, 78(4), 289-318.

Bernasconi, A. F. (1951). ber den Ossifikationsmodus bei Xenopus laevis. Daud. Denkschr. Schweiz. Mémoires de la Société des Sciences Naturelles de Neuchâtel, 79, 191-252.

Bickelmann, C., Frota-Lima, G. N., Triepel, S. K., Kawaguchi, A., Schneider, I. and Fröbisch, N. B. (2018). Noncanonical Hox, Etv4, and Gli3 gene activities give insight into unique limb patterning in salamanders. Journal of Experimental Zoology Part B: Molecular and Developmental Evolution, 330(3), 138-147. 
Blumberg, B., Bolado, J., Moreno, T. A., Kintner, C., Evans, R. M. and Papalopulu, N. (1997). An essential role for retinoid signaling in anteroposterior neural patterning. Development, 124(2), 373-379.

Bolt, J. R. (1969). Lissamphibian origins: possible protolissamphibian from the Lower Permian of Oklahoma. Science, 166(3907), 888-891.

Bolt, J. R. (1977). Dissorophoid relationships and ontogeny, and the origin of the Lissamphibia. Journal of Paleontology, 51(2), 235-249.

Bolt, J. R. (1979). Amphibamus grandiceps as a juvenile dissorophid: evidence and implications. In Mazon Creek Fossils (pp. 529-563). Cambridge: Elsevier Academic Press.

Bonebrake, J. E., and Brandon, R. A. (1971). Ontogeny of cranial ossification in the smallmouthed salamander, Ambystoma texanum (Matthes). Journal of Morphology, 133(2), 189-203.

Boncinelli, E., Simeone, A., Acampora, D. and Mavilio, F. (1991). HOX gene activation by retinoic acid. TRENDS in Genetics, 7(10), 329-334.

Bordzilovskaya, N. P., Dettlaff, T. A., Duhon, S. T., and Malacinski, G. M. (1989). Developmental-stage series of axolotl embryos. Developmental Biology of the Axolotl, 201, 219.

Bourget, H., and Anderson, J. S. (2011). A new amphibamid (Temnospondyli: Dissorophoidea) from the Early Permian of Texas. Journal of Vertebrate Paleontology, 31(1), 32-49.

Boy, J. A. (1972) Die Branchiosaurier (Amphibia) des saarpfälzischen Rotliegenden (Perm, SW-Deutschland). In Hessisches Landesamt für Bodenforschung (pp. 1-137).

Boy, J. A. (1986). Studien über die Branchiosauridae (Amphibia: Temnospondyli) 1. Neue und wenig bekannte Arten aus dem mitteleuropäischen Rotliegenden (? oberstes Karbon bis unteres Perm). Paläontologische Zeitschrift, 60(1-2), 131-166.

Boy, J. A. (1987). Studien über die Branchiosauridae (Amphibia: Temnospondyli; OberKarbon-Unter-Perm). 2. Systematische Übersicht. Neues Jahrbuch Für Geologie Und Paläontologie - Abhandlungen, 174(1), 75-104.

Boy, J. A. (1995). Über die Micromelerpetontidae (Amphibia: Temnospondyli). 1. Morphologie und Paläoökologie des Micromelerpeton credneri (Unter-Perm; SWDeutschland). Paläontologische Zeitschrift, 69(3), 429-457. 
Boy, J. A. (2002). Über die Micromelerpetontidae (Amphibia: Temnospondyli) 3. Eimerisaurus ng. Neues Jahrbuch Für Geologie Und Paläontologie - Abhandlungen, $425-452$.

Boy, J. A., and Sues, H.-D. 2000. Branchiosaurs: Larvae, metamorphosis and heterochrony in temnospondyls and seymouriamorphs. In: Heatwole H, Carroll RL, editors. Amphibian Biology, vol. 4. Chipping Norton: Surrey Beatty and Sons; pp. 1150-1197.

Brazeau, M. D., and de Winter, V. (2015). The hyoid arch and braincase anatomy of Acanthodes support chondrichthyan affinity of 'acanthodians'. Proceedings. Biological Sciences, 282(1821), http://doi.org/10.1098/rspb.2015.2210.

Brusatte, S. L., Butler, R. J., Mateus, O., and Steyer, J. S. (2015). A new species of Metoposaurus from the Late Triassic of Portugal and comments on the systematics and biogeography of metoposaurid temnospondyls. Journal of Vertebrate Paleontology, 35(3), e912988.

Burke, A. C., Nelson, C. E., Morgan, B. A., and Tabin, C. J. (1995). Hox genes and the evolution of vertebrate axial morphology. Development, 121(2), 333-346.

Butcher, E. O. (1929). The development of the somites in the white rat (Mus norvegicus albinus) and the fate of the myotomes, neural tube, and gut in the tail. American Journal of Anatomy, 44(3), 381-439.

Cardini, A., and Elton, S. (2008). Does the skull carry a phylogenetic signal? Evolution and modularity in the guenons. Biological Journal of the Linnean Society, 93(4), 813834.

Carlson, M., Komine, Y., Bryant, S. V. and Gardiner, D. M. (2001). Expression of Hoxb13 and Hoxc10 in developing and regenerating Axolotl limbs and tails. Developmental Biology, 229(2), 396-406.

Carroll, R. L. (1964). Early evolution of the dissorophid amphibians. Bulletin of the Museum of Comparative Zoology, 131, 161-250.

Carroll, R. L. (2007). The Palaeozoic ancestry of salamanders, frogs and caecilians. Zoological Journal of the Linnean Society, 150, 1-140.

Carroll, R. L., and Holmes, R. (1980). The skull and jaw musculature as guides to the ancestry of salamanders. Zoological Journal of the Linnean Society, 68(1), 1-40.

Cartry, J., Nichane, M., Ribes, V., Colas, A., Riou, J.F., Pieler, T., Dollé, P., Bellefroid, E.J. and Umbhauer, M. (2006). Retinoic acid signalling is required for specification of pronephric cell fate. Developmental Biology, 299(1), 35-51. 
Charbonneau, A. M., Roy, S., and Tran, S. D. (2016). Oral-facial tissue reconstruction in the regenerative axolotl. Journal of Experimental Zoology Part B: Molecular and Developmental Evolution, 326(8), 489-502.

Christen, B. and Slack, J. M. (1997). FGF-8 is associated with anteroposterior patterning and limb regeneration in Xenopus. Developmental Biology, 192(2), 455-466.

Chiarugi, G. (1890). Le developpement des nerfs vague, accessoire, hypoglosse et premiers cervicaux chez les sauropsides et chez les mammiferes. Archives Italiennes de Biologie, 13, 309-423.

Clack, J. A., and Holmes, R. B. (1988). The braincase of the anthracosaur Archeria crassidisca with comments on the interrelationships of primitive tetrapods. Palaeontology, 31(1), 85-107.

Clack, J. A., and Milner, A. R. (2009). Morphology and systematics of the Pennsylvanian amphibian Platyrhinops lyelli (Amphibia: Temnospondyli). Earth and Environmental Science Transactions of the Royal Society of Edinburgh, 100(3), 275-295.

Collins, J. P., Zerba, K. E., and Sredl, M. J. (1993). Shaping intraspecific variation: development, ecology and the evolution of morphology and life history variation in tiger salamanders. Genetica, 89(1-3), 167.

Conlon, R. A. and Rossant, J. (1992). Exogenous retinoic acid rapidly induces anterior ectopic expression of murine Hox-2 genes in vivo. Development, 116(2), 357-368.

Cooke, Jonathan. (1975). Control of somite number during morphogenesis of a vertebrate, Xenopus laevis. Nature, 254(5497), 196-199.

Cooke, J. and Zeeman, E. C. (1976). A clock and wavefront model for control of the number of repeated structures during animal morphogenesis. Journal of Theoretical Biology, 58(2), 455-476.

Core Team, R. (2017). R: A language and environment for statistical computing. R Foundation for Statistical Computing. Vienna, Austria: URL Https:/Www. RProject.org.

Couly, G. F., Coltey, P. M., and Le Douarin, N. M. (1993). The triple origin of skull in higher vertebrates: A study in quail-chick chimeras. Development, 117(2), 409-429.

Criswell, K. E., and Gillis, J. A. (2020). Resegmentation is an ancestral feature of the gnathostome vertebral skeleton. ELife, 9, e51696.

Daly, E. (1994). Amphibamidae (Amphibia: Temnospondyli), with a description of a new genus from the upper Pennsylvanian of Kansas. Museum of Natural History, University of Kansas, 85, 1-59. 
Dawes, B. (1930). The development of the vertebral column in mammals, as illustrated by its development in Mus musculus. Philosophical Transactions of the Royal Society of London, Series B, 218(450-461), 115-170.

de Beer, G. (1937). The development of the vertebrate skull. Oxford: Clarendon Press.

De Mar, R. E. (1967). Two new species of Broiliellus (Amphibians) from the Permian of Texas. Field Museum of Natural History.

De Mar, R. (1968). The Permian labyrinthodont amphibian Dissorophus multicinctus, and adaptations and phylogeny of the family Dissorophidae. Journal of Paleontology, 42, $1210-1242$.

de Sá, R. O., and Swart, C. C. (1999). Development of the suprarostral plate of pipoid frogs. Journal of Morphology, 240(2), 143-153.

Dempster, W. T. (1935). The brain case and endocranial cast of Eryops megacephalus (Cope). The Journal of Comparative Neurology, 62(1), 171-196.

Dequéant, M.-L., and Pourquié, O. (2008). Segmental patterning of the vertebrate embryonic axis. Nature Reviews Genetics, 9(5), 370-382.

Detwiler, S. R. (1934). An experimental study of spinal nerve segmentation in Amblystoma with reference to the plurisegmental contribution to the brachial plexus. Journal of Experimental Zoology, 67(3), 395-441.

Di Renzo, F., Broccia, M. L., Giavini, E., and Menegola, E. (2007). Citral, an inhibitor of retinoic acid synthesis, attenuates the frequency and severity of branchial arch abnormalities induced by triazole-derivative fluconazole in rat embryos cultured in vitro. Reproductive Toxicology, 24(3-4), 326-332.

Dilkes, D. W. (1990). A new trematopsid amphibian (Temnospondyli: Dissorophoidea) from the Lower Permian of Texas. Journal of Vertebrate Paleontology, 10(2), 222243.

Dockter, J. L. (2000). Sclerotome induction and differentiation. Current Topics in Developmental Biology, 48, 77-127.

Duband, J.-L., Dufour, S., Hatta, K., Takeichi, M., Edelman, G. M., and Thiery, J. P. (1987). Adhesion molecules during somitogenesis in the avian embryo. The Journal of Cell Biology, 104(5), 1361-1374.

Duellman, W. E., and Trueb, L. (1994). Biology of amphibians. New York: McGraw Hill 
Durston, A. J. (2019). What are the roles of retinoids, other morphogens, and Hox genes in setting up the vertebrate body axis? Genesis, 57(7-8), e23296.

Durston, A. J., Timmermans, J., Hage, W. J., Hendriks, H., De Vries, N. J., Heideveld, M., and Nieuwkoop, P. D. (1989). Retinoic acid causes an anteroposterior transformation in the developing central nervous system. Nature, 340, 140-144.

Durston, A. J. and Zhu, K. (2015). A time space translation hypothesis for vertebrate axial patterning. Seminars in Cell and Developmental Biology, 42, 86.

Edgeworth, F. H. (1935). The cranial muscles of vertebrates. Cambridge: Cambridge University Press.

Edwards, J. L. (1976). Spinal nerves and their bearing on, salamander phylogeny. Journal of Morphology, 148(3), 305-327.

Elliot, A. (1907). Some facts in the later development of the frog, segments of the occipital region of the skull. Quarterly Journal of Microscopical Science, 51, 647-657.

Estes, R. J. (1965). Fossil salamanders and salamander origins. American Zoologist, 5(2), 319-334.

Ferdinand, M., Z, H., Linda, Vincent, L. and Michael, S. (2006). Retinoic acid signaling and the evolution of chordates. International Journal of Biological Sciences, 2(2), 38.

Ferguson, C. A., and Graham, A. (2004). Redefining the head-trunk interface for the neural crest. Developmental Biology, 269(1), 70-80.

Filipski, G. T. and Wilson, M. V. (1984). Sudan Black B as a nerve stain for whole cleared fishes. Copeia, 204-208.

Fleming, A., Kishida, M. G., Kimmel, C. B., and Keynes, R. J. (2015). Building the backbone: the development and evolution of vertebral patterning. Development, 142(10), 1733-1744.

Fletcher, R. B., Baker, J. C. and Harland, R. M. (2006). FGF8 spliceforms mediate early mesoderm and posterior neural tissue formation in Xenopus. Development, 133(9), $1703-1714$.

Flick, R. W. (1992). Effects of Hybridization between the salamanders Ambystoma tigrinum and $A$. texanum on skull development and morphology. MA Dissertation. Carbondale: Southern Illinois University.

Frost, D. R., Grant, T., Faivovich, J., Bain, R. H., Haas, A., Haddad, C. F., et al. (2006). The amphibian tree of life. Bulletin of the American Museum of Natural History, 1291. 
Fröbisch, N. B., and Reisz, R. R. (2008). A new Lower Permian amphibamid (Dissorophoidea, Temnospondyli) from the fissure fill deposits near Richards Spur, Oklahoma. Journal of Vertebrate Paleontology, 28(4), 1015-1030.

Fröbisch, N. B., and Schoch, R. R. (2009a). Testing the impact of miniaturization on phylogeny: Paleozoic dissorophoid amphibians. Systematic Biology, 58(3), 312-327.

Fröbisch, N. B., and Schoch, R. R. (2009b). The largest specimen of Apateon and the life history pathway of neoteny in the Paleozoic temnospondyl family Branchiosauridae. Fossil Record, 12(1), 83-90.

Fürbringer, M. (1897). Uber spinooccipitalen Nerven der Selachier und Holocephalen und ihre vergleichende Morphologie. Gegenbaurs Fschr, 3, 350-788.

Gardiner, D. M., Blumberg, B., Komine, Y., and Bryant, S. V. (1995). Regulation of HoxA expression in developing and regenerating axolotl limbs. Development, 121(6), 17311741.

Gasser, R. F. (1979). Evidence that sclerotomal cells do not migrate medially during normal embryonic development of the rat. American Journal of Anatomy, 154(4), 509523.

Gaufo, G. O., Thomas, K. R., and Capecchi, M. R. (2003). Hox3 genes coordinate mechanisms of genetic suppression and activation in the generation of branchial and somatic motoneurons. Development, 130(21), 5191-5201.

Gaunt, S. J. (1994). Conservation in the Hox code during morphological evolution. International Journal of Developmental Biology, 38(3), 549-552.

Gaunt, S. J., Sharpe, P. T., and Duboule, D. (1988). Spatially restricted domains of homeogene transcripts in mouse embryos: relation to a segmented body plan. Development, 104, 169-179.

Gaunt, S. J., and Strachan, L. (1994). Forward spreading in the establishment of a vertebrate Hox expression boundary: The expression domain separates into anterior and posterior zones, and the spread occurs across implanted glass barriers. Developmental Dynamics, 199(3), 229.

Gee, B. M., Haridy, Y., and Reisz, R. R. (2017). Histological characterization of denticulate palatal plates in an Early Permian dissorophoid. PeerJ, 5, e3727.

Gee, B. M., and Reisz, R. R. (2018). Cranial and postcranial anatomy of Cacops morrisi, a eucacopine dissorophid from the early Permian of Oklahoma. Journal of Vertebrate Paleontology, 38(2), e1433186. 
Ghyselinck, N. B. and Duester, G. (2019). Retinoic acid signaling pathways. Development, 146(13), dev167502.

Gibbs, M. A. and Northcutt, R. G. (2004). Retinoic acid repatterns axolotl lateral line receptors. International Journal of Developmental Biology, 48(1), 63-66.

Gilbert, S. F. (2010). Developmental Biology, seventh edition. Sunderland: Sinauer Associates Inc.

Godsave, S., Dekker, E.-J., Holling, T., Pannese, M., Boncinelli, E., and Durston, A. (1994). Expression patterns of Hoxb genes in the Xenopus embryo suggest roles in anteroposterior specification of the hindbrain and in dorsoventral patterning of the mesoderm. Developmental Biology, 166(2), 465-476.

Godsave, S. F., Koster, C. H., Getahun, A., Mathu, M., Hooiveld, M., Van Der Wees, J., et al. (1998). Graded retinoid responses in the developing hindbrain. Developmental Dynamics, 213(1), 39-49.

Godwin, J. W., Pinto, A. R., and Rosenthal, N. A. (2013). Macrophages are required for adult salamander limb regeneration. Proceedings of the National Academy of Sciences of the United States of America, 110(23), 9415-9420.

Goodrich, E. S. (1911). On the segmentation of the occipital region of the head in the Batrachia Urodela. Proceedings of the Zoological Society of London, 1910, 101-120.

Goodrich, E. S. (1913). Memoirs: Metameric segmentation and homology. Journal of Cell Science, 2(234), 227-248.

Gomez, C., Özbudak, E. M., Wunderlich, J., Baumann, D., Lewis, J., and Pourquié, O. (2008). Control of segment number in vertebrate embryos. Nature, 454(7202), 335339.

Gomez, C., and Pourquié, O. (2009). Developmental control of segment numbers in vertebrates. Journal of Experimental Zoology Part B: Molecular and Developmental Evolution, 312(6), 533-544.

Goswami, A., and Polly, P. D. (2010). The influence of modularity on cranial morphological disparity in Carnivora and Primates (Mammalia). PLoS ONE, 5(3), e9517.

Grande, L., and Bemis, W. E. (1998). A comprehensive phylogenetic study of amiid fishes (Amiidae) based on comparative skeletal anatomy. An empirical search for interconnected patterns of natural history. Journal of Vertebrate Paleontology, 18(S1), $1-696$. 
Grinstein, M., Yelin, R., Herzlinger, D., and Schultheiss, T. M. (2013). Generation of the podocyte and tubular components of an amniote kidney: Timing of specification and a role for Wnt signaling. Development, 140(22), 4565-4573.

Gross, J. B., and Hanken, J. (2008). Segmentation of the vertebrate skull: neural-crest derivation of adult cartilages in the clawed frog, Xenopus laevis. Integrative and Comparative Biology, 48(5), 681-696.

Hall, B. K. (2005). Bones and cartilage: Developmental and evolutionary skeletal biology. Boston: Academic Press.

Handrigan, G. R., and Wassersug, R. J. (2007). The anuran bauplan: a review of the adaptive, developmental, and genetic underpinnings of frog and tadpole morphology. Biological Reviews, 82(1), 1-25.

Hanken, J., and Piekarski, N. (2013). Embryonic origin of the skull: Has the pattern of neural crest derivation changed during vertebrate evolution? The FASEB Journal, 27(1 Supplement), 315-1.

Hanken, J., and Thorogood, P. (1993). Evolution and development of the vertebrate skull: The role of pattern formation. Trends in Ecology and Evolution, 8(1), 9-15.

Harland, R. M., and Grainger, R. M. (2011). Xenopus research: metamorphosed by genetics and genomics. TRENDS in Genetics, 27(12), 507-515.

Hayashi, S., Itoh, M., Taira, S., Agata, K., and Taira, M. (2004). Expression patterns of Xenopus FGF receptor-like 1/nou-darake in early Xenopus development resemble those of planarian nou-darake and Xenopus FGF8. Developmental Dynamics, 230(4), 700-707.

Hazelton, R. D. (1970). A radioautographic analysis of the migration and fate of cells derived from the occipital somites in the chick embryo with specific reference to the development of the hypoglossal musculature. Development, 24(3), 455-466.

Herrick, C. J. (1948). The brain of the tiger salamander, Ambystoma tigrinum. Chicago: The University of Chicago Press.

Higashiyama, H., Hirasawa, T., Oisi, Y., Sugahara, F., Hyodo, S., Kanai, Y., and Kuratani, S. (2016). On the vagal cardiac nerves, with special reference to the early evolution of the head-trunk interface. Journal of Morphology, 277(9), 1146-1158.

Holmes, R. B., Carroll, R. L., and Reisz, R. R. (1998). The first articulated skeleton of Dendrerpeton acadianum (Temnospondyli, Dendrerpetontidae) from the Lower Pennsylvanian locality of Joggins, Nova Scotia, and a review of its relationships. Journal of Vertebrate Paleontology, 18(1), 64-79. 
Holmes, R., Berman, D. S., and Anderson, J. S. (2013). A new dissorophid (Temnospondyli, Dissorophoidea) from the Early Permian of New Mexico (United States). Comptes Rendus Palevol, 12(7-8), 419-435.

Hooiveld, M. H., Morgan, R., Houtzager, E., Pannese, M., Damen, K., Boncinelli, E., and Durston, A. J. (2004). Novel interactions between vertebrate Hox genes. International Journal of Developmental Biology, 43(7), 665-674.

Horan, G. S., Ramírez-Solis, R., Featherstone, M. S., Wolgemuth, D. J., Bradley, A., and Behringer, R. R. (1995). Compound mutants for the paralogous hoxa-4, hoxb-4, and hoxd-4 genes show more complete homeotic transformations and a dose-dependent increase in the number of vertebrae transformed. Genes and Development, 9(13), $1667-1677$.

Huang, R., Zhi, Q., Izpisua-Belmonte, J.-C., Christ, B., and Patel, K. (1999). Origin and development of the avian tongue muscles. Anatomy and Embryology, 200(2), 137152.

Huang, R., Zhi, Q., Patel, K., Wilting, J., and Christ, B. (2000). Contribution of single somites to the skeleton and muscles of the occipital and cervical regions in avian embryos. Journal of Anatomy and Embryology, 202(5), 375-383.

Hsiang, A. Y., Field, D. J., Webster, T. H., Behlke, A. D. B., Davis, M. B., Racicot, R. A., and Gauthier, J. A. (2015). The origin of snakes: revealing the ecology, behavior, and evolutionary history of early snakes using genomics, phenomics, and the fossil record. BMC evolutionary biology, 15(1), 1-22.

Hunter, R. M. (1935). The development of the anterior post-otic somites in the rabbit. Journal of Morphology, 57(2), 501-531.

Huelsenbeck, J. P., and Ronquist, F. (2001). MRBAYES: Bayesian inference of phylogenetic trees. Bioinformatics, 17(8), 754-755.

Hüppi, E., Núñez-León, D., Nagashima, H., and Sánchez-Villagra, M. R. (2019). Development of the chondrocranium in the domesticated fowl (Gallus gallus $f$. domestica), with a study on the variation of the of hypoglossal foramina. Vertebrate Zoology, 69(3), 299-310.

Huttenlocker, A. K., Pardo, J. D., and Small, B. J. (2007). Plemmyradytes shintoni, gen. et sp. nov., an Early Permian amphibamid (Temnospondyli: Dissorophoidea) from the Eskridge Formation, Nebraska. Journal of Vertebrate Paleontology, 27(2), 316-328.

Irving, C., and Mason, I. (2000). Signalling by $F G F 8$ from the isthmus patterns anterior hindbrain and establishes the anterior limit of Hox gene expression. Development, 127(1), 177-186. 
Ivachnenko, M. F. (1978). Urodelans from the Triassic and Jurassic of Soviet central Asia. Journal of Palaeontology, 12, 362-368.

Jenkins, F. A., Jr, Walsh, D. M., and Carroll, R. L. (2007). Anatomy of Eocaecilia micropodia, a limbed caecilian of the Early Jurassic. Bulletin of the Museum of Comparative Zoology, 158(6), 285-365.

Jones, K.E., Angielczyk, K.D., Polly, P.D., Head, J.J., Fernandez, V., Lungmus, J.K., Tulga, S. and Pierce, S.E. (2018). Fossils reveal the complex evolutionary history of the mammalian regionalized spine. Science, 361(6408), 1249-1252.

Kalcheim, C. (2011). Regulation of trunk myogenesis by the neural crest: a new facet of neural crest-somite interactions. Developmental Cell, 21(2), 187-188.

Kardong, K. V. (2015). Vertebrates: comparative anatomy, function, evolution. Boston: McGraw-Hill.

Kawahara, A., Baker, B. S., and Tata, J. R. (1991). Developmental and regional expression of thyroid hormone receptor genes during Xenopus metamorphosis. Development, 112(4), 933-943.

Kessel, M., and Gruss, P. (1990). Murine developmental control genes. Science, 249(4967), 374-379.

Kessel, M., Balling, R., and Gruss, P. (1990). Variations of cervical vertebrate after expression of a Hox-1.1 transgene in mice. Cell, 61(2), 301-308.

Kessel, M. and Gruss, P. (1991). Homeotic transformations of murine vertebrae and concomitant alteration of Hox codes induced by retinoic acid. Cell, 67(1), 89-104.

Keynes, R. J., and Stern, C. D. (1984). Segmentation in the vertebrate nervous system. Nature, 310(5980), 786-789.

Kieren, S., Sparreboom, M., Hochkirch, A., and Veith, M. (2018). A biogeographic and ecological perspective to the evolution of reproductive behaviour in the family Salamandridae. Molecular Phylogenetics and Evolution, 121, 98-109.

Kobrossy, L., Rastegar, M. and Featherstone, M. (2006). Interplay between chromatin and trans-acting factors regulating the Hoxd4 promoter during neural differentiation. Journal of Biological Chemistry, 281(36), 25926-25939.

Kolm, P. J., and Sive, H. L. (1995). Regulation of the Xenopus labial homeodomain genes, HoxAl and HoxD1: Activation by retinoids and peptide growth factors. Developmental Biology, 167(1), 34-49. 
Kondo, M., Yamamoto, T., Takahashi, S., and Taira, M. (2017). Comprehensive analyses of hox gene expression in Xenopus laevis embryos and adult tissues. Development, Growth, and Differentiation, 59(6), 526-539.

Kondo, M., Matsuo, M., Igarashi, K., Haramoto, Y., Yamamoto, T., Yasuoka, Y. and Taira, M. (2019). De novo transcription of multiple Hox cluster genes takes place simultaneously in early Xenopus tropicalis embryos. Biology Open, bio-038422.

Kragl, M., Knapp, D., Nacu, E., Khattak, S., Maden, M., Epperlein, H. H., and Tanaka, E. M. (2009). Cells keep a memory of their tissue origin during axolotl limb regeneration. Nature, 460(7251), 60 .

Kronmiller, J. E., Beeman, C. S., Nguyen, T., and Berndt, W. (1995). Blockade of the initiation of murine odontogenesis in vitro by citral, an inhibitor of endogenous retinoic acid synthesis. Archives of Oral Biology, 40(7), 645-652.

Krumlauf, R. (1994). Hox genes in vertebrate development review. Cell, 78, 191-201.

Kuratani, S. (2005). Craniofacial development and the evolution of the vertebrates: the old problems on a new background. Zoological Science, 22(1), 1-19.

Kuratani, S., Tanaka, S., Ishikawa, Y., and Zukeran, C. (1988). Early development of the hypoglossal nerve in the chick embryo as observed by the whole-mount nerve staining method. American Journal of Anatomy, 182(2), 155-168.

Kuratani, S., Horigome, N., and Hirano, S. (1999). Developmental Morphology of the Head Mesoderm and Reevaluation of Segmental Theories of the Vertebrate Head: Evidence from Embryos of an Agnathan Vertebrate, Lampetra japonica. Developmental Biology, 210(2), 381-400.

Kuratani, S., and Ota, K. G. (2008). Primitive versus derived traits in the developmental program of the vertebrate head: views from cyclostome developmental studies. Journal of Experimental Zoology Part B: Molecular and Developmental Evolution, 310(4), 294-314.

Kuratani, S., Kusakabe, R., and Hirasawa, T. (2018). The neural crest and evolution of the head/trunk interface in vertebrates. Developmental Biology, 444, S60-S66

Langston, A. W. and Gudas, L. J. (1992). Identification of a retinoic acid responsive enhancer 3' of the murine homeobox gene Hox-1.6. Mechanisms of Development, $38(3), 217-227$.

Laurin, M., and Reisz, R. R. (1997). A new perspective on tetrapod phylogeny. Amniote Origins: Completing the Transition to Land, pp. 9-59. 
Lee, M. S. Y., and Anderson, J. S. (2006). Molecular clocks and the origin(s) of modern amphibians. Molecular Phylogenetics and Evolution, 40(2), 635-639.

Lufkin, T., Mark, M., Hart, C. P., Dollé, P., LeMeur, M. and Chambon, P. (1992). Homeotic transformation of the occipital bones of the skull by ectopic expression of a homeobox gene. Nature, 359(6398), 835-841.

Lukas, P., and Olsson, L. (2018). Sequence and timing of early cranial skeletal development in Xenopus laevis. Journal of Morphology, 279(1), 62-74.

Maddin, H. C. (2015) Evolutionary morphology of the lissamphibian braincase. In: Bininda-Emonds ORP, Powell GL, Jamniczky HA, Bauer AM, editors. All animals are interesting: A festschrift in honour of Anthony P. Russell. Oldenburg: BIS-Verlag der Carl von Ossietzky Universität Oldenburg; pp. 213-243.

Maddin, H. C., Jenkins, F. A., Jr, and Anderson, J. S. (2012a). The braincase of Eocaecilia micropodia (Lissamphibia, Gymnophiona) and the origin of caecilians. PLoS ONE, 7(12), e50743.

Maddin, H. C., Russell, A. P., and Anderson, J. S. (2012b). Phylogenetic implications of the morphology of the braincase of caecilian amphibians (Gymnophiona). Zoological Journal of the Linnean Society, 166(1), 160-201.

Maddin, H. C., Fröbisch, N. B., Evans, D. C., and Milner, A. R. (2013). Reappraisal of the Early Permian amphibamid Tersomius texensis and some referred material. Comptes Rendus Palevol, 12(7), 447-461.

Maddin, H. C., Piekarski, N., Sefton, E. M., and Hanken, J. (2016). Homology of the cranial vault in birds: new insights based on embryonic fate-mapping and character analysis. Royal Society Open Science, 3(8), 160356.

Maddin, H. C., Piekarski, N., Reisz, R. R., and Hanken, J. (2020). Development and evolution of the tetrapod skull-neck boundary. Biological Reviews, 95(3), 573-591.

Maden, M. (1983). The effect of vitamin A on the regenerating axolotl limb. Development, 77(1), 273-295.

Maden, M. (2000). The role of retinoic acid in embryonic and post-embryonic development. Proceedings of the Nutrition Society, 59(1), 65-73.

Maden, M., Manwell, L. A., and Ormerod, B. K. (2013). Proliferation zones in the axolotl brain and regeneration of the telencephalon. Neural Development, 8(1), 1-15.

Maheshwar, G., Sandrine, G., K., M., Robert and François, K. (2014). Hox gene regulation in the central nervous system of Drosophila. Frontiers in Cellular Neuroscience, 8, 96. 
Mall, F. (1891). A human embryo twenty-six days old. Journal of Morphology, 5(3), 459480.

Mallo, M., Wellik, D. M., and Deschamps, J. (2010). Hox genes and regional patterning of the vertebrate body plan. Developmental Biology, 344(1), 7-15.

Mallo, M. (2016). Revisiting the involvement of signaling gradients in somitogenesis. FEBS Journal, 283(8), 1430-1437.

Manley, N. R. and Capecchi, M. R. (1997). Hox group 3 paralogous genes act synergistically in the formation of somitic and neural crest-derived structures. Developmental Biology, 192(2), 274-288.

Manzanares, M., Bel-Vialar, S., Ariza-McNaughton, L., Ferretti, E., Marshall, H., Maconochie, M. M., et al. (2001). Independent regulation of initiation and maintenance phases of Hoxa3 expression in the vertebrate hindbrain involve auto-and cross-regulatory mechanisms. Development, 128(18), 3595-3607.

Marjanovic, D., and Laurin, M. (2007). Fossils, molecules, divergence times, and the origin of lissamphibians. Systematic Biology, 56(3), 369-388.

Marjanovic, D., and Laurin, M. (2015). Reevaluation of the largest published morphological data matrix for phylogenetic analysis of Paleozoic limbed vertebrates. PeerJ PrePrints.

Marlétaz, F., Holland, L. Z., Laudet, V., and Schubert, M. (2006). Retinoic acid signaling and the evolution of chordates. International Journal of Biological Sciences, $2(2), 38$.

Mark, M., Ghyselinck, N. B., and Chambon, P. (2009). Function of retinoic acid receptors during embryonic development. Nuclear Receptor Signaling, 7(1), nrs-07002.

Marshall, H., Studer, M., Pöpperl, H., Aparicio, S., Kuroiwa, A., Brenner, S., and Krumlauf, R. (1994). A conserved retinoic acid response element required for early expression of the homeobox gene Hoxb-1. Nature, 370(6490), 567.

Matsuoka, T., Ahlberg, P. E., Kessaris, N., Iannarelli, P., Dennehy, U., Richardson, W. D., et al. (2005). Neural crest origins of the neck and shoulder. Nature, 436(7049), 347355.

Massidon, W. P., and Maddison, D. R. (2018). Mesquite: a modular system for evolutionary analysis. Version 3.51.

McGinnis, W., and Krumlauf, R. (1992). Homeobox genes and axial patterning. Cell, 68(2), 283-302. 
McNulty, C. L., Peres, J. N., Bardine, N., Akker, W. M. R. van den and Durston, A. J. (2005). Knockdown of the complete Hox paralogous group 1 leads to dramatic hindbrain and neural crest defects. Development, 132(12), 2861-2871.

Merilä, J., Laurila, A., Pahkala, M., Räsänen, K., and Timenes Laugen, A. (2000). Adaptive phenotypic plasticity in timing of metamorphosis in the common frog Rana temporaria. Ecoscience, 7(1), 18-24.

Milner, A. R. 1988. The relationships and origin of living amphibians. In: Benton MJ, editor. The phylogeny and classification of the tetrapods 1 . Amphibians, reptiles, birds. Oxford: Oxford University Press; pp. 59-102.

Milner, A. R. (1993). The Paleozoic relatives of lissamphibians. Herpetological Monographs, 7, 8-27.

Milner, A. R., and Schoch, R. R. (2013). Trimerorhachis (Amphibia: Temnospondyli) from the Lower Permian of Texas and New Mexico: cranial osteology, taxonomy and biostratigraphy. Neues Jahrbuch Für Geologie Und Paläontologie - Abhandlungen, 270(1), 91-128.

Mitgutsch, C., Piekarski, N., Olsson, L., and Haas, A. (2008). Heterochronic shifts during early cranial neural crest cell migration in two ranid frogs. Acta Zoologica, 89(1), 6978.

Monaghan, J. R. and Maden, M. (2012). Visualization of retinoic acid signaling in transgenic axolotls during limb development and regeneration. Developmental Biology, 368(1), 63-75.

Montavon, T., and Soshnikova, N. (2014). Hox gene regulation and timing in embryogenesis. Seminars in Cell and Developmental Biology, 34, 76-84.

Monteiro, A. S., and Ferrier, D. E. (2006). Hox genes are not always colinear. International Journal of Biological Sciences, 2(3), 95.

Moore, M. K. (1991). Comparative ontogeny of cranial ossification in the spotted salamander, Ambystoma maculatum, and the tailed frog, Ascaphus truei. MSc Dissertation. Baton Rouge: Louisiana State University.

Müller, H. (2006). Ontogeny of the skull, lower jaw, and hyobranchial skeleton of Hypogeophis rostratus (Amphibia: Gymnophiona: Caeciliidae) revisited. Journal of Morphology, 267(8), 968-986.

Naumann, B., and Olsson, L. (2018). Three-dimensional reconstruction of the cranial and anterior spinal nerves in early tadpoles of Xenopus laevis (Pipidae, Anura). Journal of Comparative Neurology, 526(5), 836-857. 
Nieuwkoop, P. D., and Faber, J. (1994). Normal Table of Xenopus laevis (Daudin). New York: Garland Publishing.

Noden, D. M. (1983). The embryonic origins of avian cephalic and cervical muscles and associated connective tissues. The American Journal of Anatomy, 168(3), 257-276.

Nolte, C., Kumar, B. D. and Krumlauf, R. (2019). Hox genes: Downstream "effectors" of retinoic acid signaling in vertebrate embryogenesis. Genesis, 57(7-8), e23306.

Norris, H. W. (1908). The cranial nerves of Amphiuma means. Journal of Comparative Neurology and Psychology, 18(6), 527-568.

Norris, H. W. (1913). The cranial nerves of Siren lacertina. Journal of Morphology, 24(2), 245-338.

Northcutt, R. G., Catania, K. C., and Criley, B. B. (1994). Development of lateral line organs in the axolotl. Journal of Comparative Neurology, 340(4), 480.

Northcutt, R. G., Barlow, L. A., Braun, C. B., and Catania, K. C. (2000). Distribution and innervation of taste buds in the axolotl. Brain, Behavior and Evolution, 56(3), 123145.

Nowicki, J. L., and Burke, A. C. (2000). Hox genes and morphological identity: axial versus lateral patterning in the vertebrate mesoderm. Development, 127(19), 42654275.

Nye, H. L., Cameron, J. A., Chernoff, E. A., and Stocum, D. L. (2003). Extending the table of stages of normal development of the axolotl: limb development. Developmental Dynamics, 226(3), 555-560.

O'Rahilly, R., and Meyer, D. B. (1979). The timing and sequence of events in the development of the human vertebral column during the embryonic period proper. Anatomy and Embryology, 157(2), 167-176.

Oisi, Y., Ota, K. G., Fujimoto, S. and Kuratani, S. (2013). Development of the chondrocranium in hagfishes, with special reference to the early evolution of vertebrates. Zoological Science, 30(11), 944-961.

Oisi, Y., Fujimoto, S., Ota, K. G. and Kuratani, S. (2015). On the peculiar morphology and development of the hypoglossal, glossopharyngeal and vagus nerves and hypobranchial muscles in the hagfish. Zoological Letters, 1(1), 1-15.

Olsson, L., Ericsson, R., and Cerny, R. (2005). Vertebrate head development: Segmentation, novelties, and homology. Theory in Biosciences, 124(2), 145-163. 
Papalopulu, N., and Kintner, C. (1996). A posteriorising factor, retinoic acid, reveals that anteroposterior patterning controls the timing of neuronal differentiation in Xenopus neuroectoderm. Development, 122(11), 3409-3418.

Pardo, J. D., Small, B. J., and Huttenlocker, A. K. (2017). Stem caecilian from the Triassic of Colorado sheds light on the origins of Lissamphibia. Proceedings of the National Academy of Sciences, 114(27), E5389-E5395.

Parker, W. K. (1876). XXIV. On the structure and development of the skull in the batrachia.-Part II. Philosophical Transactions of the Royal Society of London, 166, 601-669.

Pearson, J. C., Lemons, D., and McGinnis, W. (2005). Modulating Hox gene functions during animal body patterning. Nature Reviews Genetics, 6(12), 893-904.

Pereyra, M. O., Womack, M. C., Barrionuevo, J. S., Blotto, B. L., Baldo, D., Targino, M., et al. (2016). The complex evolutionary history of the tympanic middle ear in frogs and toads (Anura). Scientific Reports, 6, 1-9.

Pérez-Ben, C. M., Schoch, R. R., and Báez, A. M. (2018). Miniaturization and morphological evolution in Paleozoic relatives of living amphibians: a quantitative approach. Paleobiology, 44(1), 58-75.

Piekarski, N. (2009). A long-term fate map of the anterior somites in the Mexican axolotl (Ambystoma mexicanum). PhD Dissertation. Jena: Friedrich-Schiller-Universität.

Piekarski, N., and Olsson, L. (2007). Muscular derivatives of the cranialmost somites revealed by long-term fate mapping in the Mexican axolotl (Ambystoma mexicanum). Evolution and Development, 9(6), 566-578.

Piekarski, N., and Olsson, L. (2014). Resegmentation in the Mexican axolotl, Ambystoma mexicanum. Journal of Morphology, 275(2), 141-152.

Piekarski, N., Gross, J. B., and Hanken, J. (2014). Evolutionary innovation and conservation in the embryonic derivation of the vertebrate skull. Nature Communications, 5(1), 1-9.

Platt, J. B. (1897). The development of the cartilaginous skull and of the branchial and hypoglossal musculature in Necturus. Morphologisches Jahrbuch, 25, 377-464.

Polley, B. P., and Reisz, R. R. (2011). A new Lower Permian trematopid (Temnospondyli: Dissorophoidea) from Richards Spur, Oklahoma. Zoological Journal of the Linnean Society, 161(4), 789-815

Pourquié, O. (2003). The segmentation clock: Converting embryonic time into spatial pattern. Science, 301(5631), 328-330. 
Pyron, R. A., and Wiens, J. J. (2013). Large-scale phylogenetic analyses reveal the causes of high tropical amphibian diversity. Proceedings of the Royal Society B: Biological Sciences, 280(1770), 20131622.

Quinzio, S. I., and Fabrezi, M. (2019). The peripheral nerves of Lepidobatrachus tadpoles (Anura, Ceratophryidae). Journal of Morphology, 280(1), 4-19.

Rage, J.-C., and Janvier, P. (1982). Le problème de la monophylie des amphibiens actuels, a la lumière des nouvelles données sur les affinités des tétrapodes. Geobios, 15, 6583.

Rage, J.-C., and Rocek, Z. (1989). Redescription of Triadobatrachus massinoti (Piveteau, 1936) an anuran amphibian from the early Triassic. Palaeontographica A, 206, 1-16.

Rambaut, A., Suchard, M. A., Xie, D., and Drummond, A. J. (2015). Tracer v1. 6. 2014.

Ramírez-Solis, R., Zheng, H., Whiting, J., Krumlauf, R., and Bradley, A. (1993). Hoxb-4 (Hox-2.6) mutant mice show homeotic transformation of a cervical vertebra and defects in the closure of the sternal rudiments. Cell, 73(2), 279-294.

Rastegar, M., Kobrossy, L., Kovacs, E. N., Rambaldi, I. and Featherstone, M. (2004). Sequential histone modifications at Hoxd4 regulatory regions distinguish anterior from posterior embryonic compartments. Molecular and Cellular Biology, 24(18), 80908103.

Reilly, S. M. (1987). Ontogeny of the hyobranchial apparatus in the salamanders Ambystoma talpoideum (Ambystomatidae) and Notophthalmus viridescens (Salamandridae): the ecological morphology of two neotenic strategies. Journal of Morphology, 191(2), 205-214.

Reisz, R. R., Schoch, R. R., and Anderson, J. S. (2009). The armoured dissorophid Cacops from the Early Permian of Oklahoma and the exploitation of the terrestrial realm by amphibians. Die Naturwissenschaften., 96(7), 789-796.

Remak, R. (1855). Untersuchungen über die Entwicklung der Wirbelthiere, Berlin: Reimer.

Revell, L. J. (2012). Phytools: An R package for phylogenetic comparative biology (and other things). Methods in Ecology and Evolution, 3(2), 217-223.

Richardson, M. K., Allen, S. P., Wright, G. M., Raynaud, A., and Hanken, J. (1998). Somite number and vertebrate evolution. Development, 125(2), 151-160. 
Rieden, P. M. J. (2003). Control of Hox gene regulation and function during anteroposterior patterning in Xenopus laevis. PhD Dissertation. Utrecht: Nederlands Instituut voor Ontwikkelingsbiologie,

Robinson, J., Ahlberg, P. E., and Koentges, G. (2005). The braincase and middle ear region of Dendrerpeton acadianum (Tetrapoda: Temnospondyli). Zoological Journal of the Linnean Society, 143(4), 577-597.

Romer, A. S. (1946). The primitive reptile Limnoscelis restudied. American Journal of Science, 244(3), 149-188.

Romer, A. S., and Edinger, T. (1942). Endocranial casts and brains of living and fossil Amphibia. Journal of Comparative Neurology, 77(2), 355-389.

Romer, A. S., and Witter, R. V. (1942). Edops, a primitive rhachitomous amphibianfrom the Texas red beds. The Journal of Geology, 50(8), 925-960.

Romer, A. S., and Parsons, T. S. (1977). The vertebrate body. Philadelphia: Saunders.

Rose, C. S. (2003). The developmental morphology of salamander skulls. In H. Heatwole and M. Davies (Eds.), Amphibian biology (Vol. 5, pp. 1684-1781). Chipping Norton (Australia): Surrey Beatty and sons.

Roth, G., Wake, D. B., Wake, M. H., and Rettig, G. (1984). Distribution of accessory and hypoglossal nerves in the hindbrain and spinal cord of lungless salamanders, family Plethodontidae. Neuroscience Letters, 44(1), 53-57.

Ruiz i Altaba, A. R., and Jessell, T. (1991). Retinoic acid modifies mesodermal patterning in early Xenopus embryos. Genes and Development, 5(2), 175-187.

Ruta, M., and Bolt, J. R. (2006). A reassessment of the temnospondyl amphibian Perryella olsoni from the Lower Permian of Oklahoma. Earth and Environmental Science Transactions of the Royal Society of Edinburgh, 97(2), 113-165.

Ruta, M., and Coates, M. I. (2007). Dates, nodes and character conflict: addressing the lissamphibian origin problem. Journal of Systematic Palaeontology, 5(01), 69-122.

Sader, F., Denis, J. F., and Roy, S. (2018). Tissue regeneration in dentistry: Can salamanders provide insight? Oral Diseases, 24(4), 509-517.

Sakamoto, Y. (2019). Morphological features of the branching pattern of the hypoglossal nerve. The Anatomical Record, 302(4), 558-567.

Samuels, M. E., Regnault, S., and Hutchinson, J. R. (2017). Evolution of the patellar sesamoid bone in mammals. PeerJ, 5, e3103. 
Sasson, D. A., and Ryan, J. F. (2017). A reconstruction of sexual modes throughout animal evolution. BMC Evolutionary Biology, 17(1), 242.

Scaal, M. (2016). Early development of the vertebral column. Seminars in Cell and Developmental Biology, 49, 83-91.

Scadding, S. R. (1999). Citral, an inhibitor of retinoic acid synthesis, modifies pattern formation during limb regeneration in the axolotl Ambystoma mexicanum. Canadian Journal of Zoology, 77(11), 1835-1837.

Schoch, R. R. (1999). Studies on braincases of early tetrapods: structure, morphological diversity, and phylogeny-2. Dissorophoids, eryopids, and stereospondyls. Neues Jahrbuch Für Geologie Und Paläontologie - Abhandlungen, 289-312.

Schoch, R. R. (2002). The neurocranium of the stereospondyl Mastodonsaurus giganteus. Palaeontology, 45(3), 627-645.

Schoch, R. R. (2012). Character distribution and phylogeny of the dissorophid temnospondyls. Fossil Record, 15(2), 121-137.

Schoch, R. R. (2013a). How body size and development biased the direction of evolution in early amphibians. Historical Biology, 25(2), 155-165.

Schoch, R. R. (2013b). The evolution of major temnospondyl clades: an inclusive phylogenetic analysis. Journal of Systematic Palaeontology, 11(6), 673-705.

Schoch, R. R. (2014a). Amphibian skull evolution: The developmental and functional context of simplification, bone loss and heterotopy. Journal of Experimental Zoology Part B: Molecular and Developmental Evolution, 322(8), 619-630.

Schoch, R. R. (2014b). First evidence of the branchiosaurid temnospondyl Leptorophus in the Early Permian of the Saar-Nahe Basin (SW Germany). Neues Jahrbuch für Geologie und Paläontologie-Abhandlungen, 272(2), 225-236.

Schoch, R. R. (2017). The stapes of Edops craigi and ear evolution in the lissamphibian stem group. Acta Zoologica, 100(2), 126-134

Schoch, R. R. (2018). The putative lissamphibian stem-group: phylogeny and evolution of the dissorophoid temnospondyls. Journal of Paleontology, 1-20.

Schoch, R. R., and Milner, A. R. (2004). Structure and implications of theories on the origin of lissamphibians. Recent advances in the origin and early radiation of vertebrates, 345-377. 
Schoch, R. R., and Rubidge, B. S. (2005). The amphibamid Micropholis from the Lystrosaurus assemblage zone of South Africa. Journal of Vertebrate Paleontology, 25(3), 502-522.

Schoch, R. R., and Fröbisch, N. (2006). Metamorphosis and neoteny: alternative pathways in an extinct amphibian clade. Evolution, 60(7), 1467-1475.

Schoch, R. R., and Milner, A. R. (2008). The intrarelationships and evolutionary history of the temnospondyl family Branchiosauridae. Journal of Systematic Palaeontology Journal of Systematic Palaeontology, 6(4), 409-431.

Schoch, R. R., and Witzmann, F. (2009). Osteology and relationships of the temnospondyl genus Sclerocephalus. Zoological Journal of the Linnean Society, 157(1), 135-168.

Schoch, R. R., and Sues, H.-D. (2013). A new dissorophid temnospondyl from the Lower Permian of north-central Texas. Comptes Rendus Palevol, 12(7-8), 437-445.

Schoch, R. R. and Milner, A. R. (2014). Handbuch Der Paläoherpetologie: Encyclopedia of Paleoherpetology. Temnospondyli I/by Rainer R. Schoch and Andrew R. Milner. Pfeil.

Scholsser, G., and Roth, G. (1995). Distribution of cranial and rostral spinal nerves in tadpoles of the frog Discoglossus pictus (Discoglossidae). Journal of Morphology, 226(2), 189-212.

Schreckenberg, G. M., and Jacobson, A. G. (1975). Normal stages of development of the axolotl, Ambystoma mexicanum. Developmental Biology, 42(2), 391-399.

Schröter, C., Herrgen, L., Cardona, A., Brouhard, G. J., Feldman, B., and Oates, A. C. (2008). Dynamics of zebrafish somitogenesis. Developmental Dynamics, 237(3), 545553.

Schuh, T. J., Hall, B. L., Kraft, J. C., Privalsky, M. L., and Kimelman, D. (1993). v-erbA and citral reduce the teratogenic effects of all-trans retinoic acid and retinol, respectively, in Xenopus embryogenesis. Development, 119(3), 785-798.

Sefton, E. M., Piekarski, N., and Hanken, J. (2015). Dual embryonic origin and patterning of the pharyngeal skeleton in the axolotl (Ambystoma mexicanum). Evolution and Development, 17(3), 175-184.

Sensenig, E. C. (1949). The early development of the human vertebral column. Contributions to Embryology, 33, 23-40.

Sheil, C. A., Jorgensen, M., Tulenko, F., and Harrington, S. (2014). Variation in timing of ossification affects inferred heterochrony of cranial bones in Lissamphibia. Evolution and Development, 16(5), 292-305. 
Sigurdsen, T., and Bolt, J. R. (2010). The Lower Permian amphibamid Doleserpeton (Temnospondyli: Dissorophoidea), the interrelationships of amphibamids, and the origin of modern amphibians. Journal of Vertebrate Paleontology, 30(5), 1360-1377.

Sive, H. L., Draper, B. W., Harland, R. M., and Weintraub, H. (1990). Identification of a retinoic acid-sensitive period during primary axis formation in Xenopus laevis. Genes and Development, 4(6), 932-942.

Skutschas, P., and Martin, T. (2011). Cranial anatomy of the stem salamander Kokartus honorarius (Amphibia: Caudata) from the Middle Jurassic of Kyrgyzstan. Zoological Journal of the Linnean Society, 161(4), 816-838.

Slater, B. J., Liu, K. J., Kwan, M. D., Quarto, N., and Longaker, M. T. (2009). Cranial osteogenesis and suture morphology in Xenopus laevis: a unique model system for studying craniofacial development. PloS one, 4(1), e3914.

Smirnov, S. V., Vassilieva, AB. (2005). Skull development in normal, TH-exposed, and goitrogen-treated axolotls, Ambystoma mexicanum. Russian Journal of Herpetology, $12,113-126$.

Strong, O. S. (1895). The cranial nerves of amphibia. A contribution to the morphology of the vertebrate nervous system. PhD Dissertation. Boston: Columbia College.

Stuesse, S. L., Cruce, W. L., and Powell, K. S. (1983). Afferent and efferent components of the hypoglossal nerve in the grass frog, Rana pipiens. The Journal of Comparative Neurology, 217(4), 432-439.

Sumida, S. S., Berman, D. S., and Martens, T. (1998). A new trematopid amphibian from the Lower Permian of central Germany. Palaeontology, 41(4), 605-630.

Swindell, E. C. and Eichele, G. (1999). Retinoid metabolizing enzymes in development. Biofactors, 10(2-3), 85-89.

Swofford, D. L., and Sullivan, J. (2003). Phylogeny inference based on parsimony and other methods using PAUP*. The Phylogenetic Handbook: a Practical Approach to DNA and Protein Phylogeny, Cáp, 7, 160-206.

Tam, P. (1981). The control of somitogenesis in mouse embryos. Journal of Embryology, $65,103-128$.

Tanaka, M., Tamura, K. and Ide, H. (1996). Citral, an inhibitor of retinoic acid synthesis, modifies chick limb development. Developmental Biology, 175(2), 239-247. 
Torok, M. A., Gardiner, D. M., Shubin, N. H., and Bryant, S. V. (1998). Expression of HoxD genes in developing and regenerating axolotl limbs. Developmental Biology, 200(2), 225-233.

Trueb, L., and Cloutier, R. (1991). A phylogenetic investigation of the inter-and intrarelationships of the Lissamphibia (Amphibia: Temnospondyli). Origins of the Higher Groups of Tetrapods: Controversy and Consensus, 223-313.

Trueb, L., and Hanken, J. (1992). Skeletal development in Xenopus laevis (Anura: Pipidae). Journal of Morphology, 214(1), 1-41.

Trueb, L. (1993). Patterns of cranial diversity among the Lissamphibia. In: Hanken J, Hall, BK, editors. The Skull, Vol. 2, Patterns of Structural and Systematic Diversity: Chicago: The University of Chicago Press; pp. 255-343.

Trueb, L., Púgener, L. A., and Maglia, A. M. (2000). Ontogeny of the bizarre: an osteological description of Pipa pipa (Anura: Pipidae), with an account of skeletal development in the species. Journal of Morphology, 243(1), 75-104.

Vallin, G., and Laurin, M. (2004). Cranial morphology and affinities of Microbrachis, and a reappraisal of the phylogeny and lifestyle of the first amphibians. Journal of Vertebrate Paleontology, 24(1), 56-72.

von Ebner, V. (1888). Urwirbel und neugliederung der wirbelsäule. Sitzungsber Akad Wiss Wien, 3(97), 194-206.

Wacker, S. A., McNulty, C. L. and Durston, A. J. (2004). The initiation of Hox gene expression in Xenopus laevis is controlled by Brachyury and BMP-4. Developmental Biology, 266(1), 123-137.

Wake, D. B., and Lawson, R. (1973). Developmental and adult morphology of the vertebral column in the plethodontid salamander Eurycea bislineata, with comments on vertebral evolution in the Amphibia. Journal of Morphology, 139(3), 251-299.

Wake, M. H. (1992). Patterns of peripheral innervation of the tongue and hyobranchial apparatus in caecilians (Amphibia: Gymnophiona). Journal of Morphology, 212(1), $37-53$.

Wake, M. H. (1993). Evolutionary diversification of cranial and spinal nerves and their targets in the gymnophione amphibians. Acta Anatomica, 148(2-3), 160-168.

Ward, L., Evans, S. E., and Stern, C. D. (2017). A resegmentation-shift model for vertebral patterning. Journal of Anatomy, 230(2), 290-296.

Wellik, D. M., and Capecchi, M. R. (2003). Hox10 and Hox11 genes are required to globally pattern the mammalian skeleton. Science, 301(5631), 363-367. 
Werneburg, R. (1994). Dissorophoiden (Amphibia, Rhachitomi) aus dem Westfal D (Oberkarbon) von Böhmen-Limnogyrinus elegans (Fritsch 1881). Zeitschrift Fur Geologische Wissenschaften, 22, 457-457.

Werneburg, R., Ronchi, A., and Schneider, J. W. (2007). The Early Permian branchiosaurids (Amphibia) of Sardinia (Italy): systematic palaeontology, palaeoecology, biostratigraphy and palaeobiogeographic problems. Palaeogeography, Palaeoclimatology, Palaeoecology Palaeogeography, Palaeoclimatology, Palaeoecology, 252(3-4), 383-404.

Werneburg, R., Lucas, S. G., Schneider, J. W., and Rinehart, L. F. (2010). First Pennsylvanian Eryops (Temnospondyli) and its Permian record from New Mexico. New Mexico Museum of Natural History and Science Bulletin, 49, 129-135.

Williston, S. W. (1910). Cacops, Desmospondylus; new genera of Permian vertebrates. Bulletin of the Geological Society of America, 21(1), 249-284.

Williston, S. W. (1914). Broiliellus, a new genus of amphibians from the Permian of Texas. The Journal of Geology, 22(1), 49-56.

Witzmann, F. (2006). Developmental patterns and ossification sequence in the PermoCarboniferous temnospondyl Archegosaurus decheni (Saar-Nahe Basin, Germany). Journal of Vertebrate Paleontology, 26(1), 7-17.

Witzmann, F., Schoch, R. R., Hilger, A., and Kardjilov, N. (2012). Braincase, palatoquadrate and ear region of the plagiosaurid Gerrothorax pulcherrimus from the Middle Triassic of Germany. Palaeontology, 55(1), 31-50.

Wobus, A. M., Kaomei, G., Shan, J., Wellner, M.-C., Rohwedel, J., Guanju, J., Fleischmann, B., Katus, H. A., Hescheler, J. and Franz, W.-M. (1997). Retinoic acid accelerates embryonic stem cell-derived cardiac differentiation and enhances development of ventricular cardiomyocytes. Journal of Molecular and Cellular Cardiology, 29(6), 1525-1539.

Woltering, J. M., Holzem, M. and Meyer, A. (2019). Lissamphibian limbs and the origins of tetrapod hox domains. Developmental Biology, 456(2), 138-144.

Yang, E. V., Gardiner, D. M., Carlson, M. R., Nugas, C. A., and Bryant, S. V. (1999). Expression of Mmp-9 and related matrix metalloproteinase genes during axolotl limb regeneration. Developmental Dynamics, 216(1), 2-9.

Yaryhin, O., and Werneburg, I. (2018). Tracing the developmental origin of a lizard skull: Chondrocranial architecture, heterochrony, and variation in lacertids. Journal of Morphology, 279(8), 1058-1087. 
Yau, T. O., Kwan, C. T., Jakt, L. M., Stallwood, N., Cordes, S., and Sham, M. H. (2002). Auto/cross-regulation of Hoxb3 expression in posterior hindbrain and spinal cord. Developmental Biology, 252(2), 287-300.

Youn, B. W., and Malacinski, G. M. (1981). Somitogenesis in the amphibian Xenopus laevis: scanning electron microscopic analysis of intrasomitic cellular arrangements during somite rotation. Development, 64(1), 23-43.

Zahn, N., Levin, M., and Adams, D. S. (2017). The Zahn drawings: new illustrations of Xenopus embryo and tadpole stages for studies of craniofacial development. Development, 144(15), 2708-2713.

Zelditch, M. L., and Fink, W. L. (1996). Heterochrony and heterotopy: Stability and innovation in the evolution of form. Paleobiology, 22(2), 241-254.

Zhu, K., Spaink, H. P. and Durston, A. J. (2017). Hoxc6 loss of function truncates the main body axis in Xenopus. Cell Cycle, 16(11), 1136-1138.

Ziermann, J. M., and Olsson, L. (2007). Patterns of spatial and temporal cranial muscle development in the African clawed frog, Xenopus laevis (Anura: Pipidae). Journal of Morphology, 268(9), 791-804. 